\title{
Barium Ion Extraction and Identification from Laser Induced Fluorescence in Gas for the Enriched Xenon Observatory
}

by

\author{
Etienne Rollin
}

A thesis submitted to the Faculty of Graduate and Postdoctoral Affairs in partial fulfillment of the requirements for the degree of

Doctor of Philosophy

in

Physics

Carleton University

Ottawa, Ontario, Canada

(C) 2011

Etienne Rollin 
Library and Archives

Canada

Published Heritage

Branch

395 Wellington Street

Ottawa ON K1A ON4

Canada
Bibliotheqque et

Archives Canada

Direction du

Patrimoine de l'édition

395 , rue Wellington

Ottawa ON K1A ON4

Canada
Your file Votre référence

ISBN: 978-0-494-81581-6

Our file Notre référence

ISBN: 978-0-494-81581-6
NOTICE:

The author has granted a nonexclusive license allowing Library and Archives Canada to reproduce, publish, archive, preserve, conserve, communicate to the public by telecommunication or on the Internet, loan, distribute and sell theses worldwide, for commercial or noncommercial purposes, in microform, paper, electronic and/or any other formats.

The author retains copyright ownership and moral rights in this thesis. Neither the thesis nor substantial extracts from it may be printed or otherwise reproduced without the author's permission.
AVIS:

L'auteur a accordé une licence non exclusive permettant à la Bibliothèque et Archives Canada de reproduire, publier, archiver, sauvegarder, conserver, transmettre au public par télécommunication ou par l'Internet, prêter, distribuer et vendre des thèses partout dans le monde, à des fins commerciales ou autres, sur support microforme, papier, électronique et/ou autres formats.

L'auteur conserve la propriété du droit d'auteur et des droits moraux qui protège cette thèse. Ni la thèse ni des extraits substantiels de celle-ci ne doivent être imprimés ou autrement reproduits sans son autorisation.
In compliance with the Canadian Privacy Act some supporting forms may have been removed from this thesis.

While these forms may be included in the document page count, their removal does not represent any loss of content from the thesis.
Conformément à la loi canadienne sur la protection de la vie privée, quelques formulaires secondaires ont été enlevés de cette thèse.

Bien que ces formulaires aient inclus dans la pagination, il n'y aura aucun contenu manquant.

\section{Canadä}




\section{Abstract}

The scientific community is increasingly interested in neutrinoless double beta decay. A potential measurement of the decay rate would determine the neutrino mass and would be sensitive to some extensions of the Standard Model of particle physics. Unfortunately, the decay rate is very low and competes with natural and cosmogenic radioactivity. This thesis presents a technique that eliminates such background events. It is performed by observing the barium ion daughter from the double beta decay of xenon-136 using laser induced fluorescence. The technique is very complex and requires an excellent understanding of the barium ion spectroscopy and its chemistry in the vicinity of other molecules. Such a technique will become a unique advantage over other neutrinoless double beta decay experiments, especially if the neutrino mass is low.

This thesis describes three main topics. The first one describes simulations of ionizing electrons in xenon to determine the size of a gas phase detector for a neutrinoless double beta decay measurement. It has been determined that a meter size detector would contain most electron tracks. Then, it describes the design of two barium ion sources, one relying on electric discharges across two electrodes and the other one using a high energy pulsed laser. From those sources, the spectroscopy of barium ions was studied. The branching ratio of the $6^{2} S_{1 / 2}-$ $6^{2} P_{1 / 2}$ transition was found to be $74 \pm 4 \%$. By adding argon in the chamber, the lineshift of the transition due to collisions was found to be $-132 \mathrm{MHz} /$ torr while the broadening rate was $23 \mathrm{MHz} /$ torr. Finally, the most interesting topic is the production of doubly charged barium ions using an electrospray source. From it, ions were extracted to vacuum in a mass spectrometer and charge conversion was achieved using triethylamine. The efficiency of the conversion of $\mathrm{Ba}^{+}$to $\mathrm{Ba}^{++}$was almost $100 \%$, with a cross-section between $1.69 \times 10^{-18} \mathrm{~m}^{2}$ and $2.21 \times 10^{-18} \mathrm{~m}^{2}$ without forming any molecules. 


\section{Acknowledgements}

This thesis would not have been possible without the counselling and patience of my two supervisors, Dr. David Sinclair and Dr. Kevin Graham. They deserve additional gratitude for allowing me to work on the very entertaining barium tagging concept. I must also acknowledge my former Master degree supervisor Dr. Alain Bellerive who taught me the basis of research. I need to praise the generosity of Dr. Jeffrey Smith who provided the mass spectrometer described in Chapter 5, the core of the thesis. Special thanks to Dr. Madhu Dixit who allowed me to visit the KEK accelerator in Japan. I would like to express my gratitude to the very skillful technical staff Philippe Gravelle, Lina Anselmo, Matt Bowcock, Yves Baribeau and Ernie Neuheimer who spent countless hours helping me. I am indebted to the thesis examination board that consisted of Dr. Ken Ragan, Dr. Jeff Smith, Dr. Gerald Oakham, Dr. James Harden and Dr. Michel Gaulin for the very careful reading of the preliminary version of my thesis and their valuable comments. My thanks to my co-workers and friends Olivier Simard, Pierre-Luc Drouin, Chad Greene, Christina Hägemann, Christian Ouellet, Alex Coppens, François Léonard, Matt Dunford, John-Paul Archambault, Claudiu Cojocaru, Ken Moats, Louise Heelan, Maria Paula Rozo, Lisa Kaufman and Jesse Wodin. Some of them contributed directly to this thesis while others have helped to make the last few years more enjoyable.

Finallement, je dédie cette thèse à ma famille, mon père Rémi Rollin, ma mère Cécile Laverdière, mes frères Jean-Philip et David Rollin, ainsi qu'aux deux

personnes les plus importantes dans ma vie, ma femme Katia Larrivée et ma fille Amélie Rollin-Larrivée. Sans votre support et votre patience, ce travail n'aurait pas été possible. Tout l'honneur vous revient. 


\section{Statement of Originality}

I certify that this thesis is the product of my own work, and that any ideas or quotations from the work of other people are fully acknowledged in accordance with the standard referencing practises of the discipline. I acknowledge the helpful guidance an support of my supervisors Dr. David Sinclair and Dr. Kevin Graham.

The analysis described in Section 2.3.2 is based on the theoretical developments of Ali et al. and my contribution was to apply their equations to a tracking detector for a specific $0 \nu \beta \beta$ decay model. Track simulations of Chapter 3 were performed by myself using the well developed GEANT4 simulation package. My work helped to decide on the final dimension and geometry of the XEP chamber. Chapter 4 describes two ion sources. The design and the assembly of them were performed by myself, Matt Bowcock and Philippe Gravelle, with the valuable advice of Dr. David Sinclair. I performed the final assembly, installation and commissioning at SNOLab. The data acquired was the result of my manipulation with the assistance of Lina Anselmo while the analysis was my own work. Finally, Chapter 5 reports my work performed on barium tagging using a commercial instrument. Several people helped me to repair and modernize it and I performed acquisition and analysis of the data. My work allowed to find a way to efficiently convert $\mathrm{Ba}^{++}$to $\mathrm{Ba}^{+}$and to lay out the basis of barium tagging for a gas phase EXO experiment.

During my Doctor of Philosophy degree, I signed eight refereed articles and conference proceedings:

1. Electron energy spectra, fluxes, and day-night asymmetries of B-8 solar neutrinos from measurements with $\mathrm{NaCl}$ dissolved in the heavy-water detector at the Sudbury Neutrino Observatory SNO Collaboration, Phys. Rev. C72, 055502 (2005). 
2. A Search for periodicities in the B-8 solar neutrino flux measured by the Sudbury Neutrino Observatory, SNO Collaboration, Phys. Rev. D72, 052010 (2005).

3. Spatial resolution of a micromegas-TPC using the charge dispersion signal, A. Bellerive et al., Proceedings of 2005 International Linear Collider Workshop (LCWS 2005), Stanford, California, 18-22 Mar 2005.

4. A Search for Neutrinos from the Solar hep Reaction and the Diffuse Supernova Neutrino Background with the Sudbury Neutrino Observatory, SNO Collaboration, Astrophys. J. 653, 1545 (2006).

5. An Independent Measurement of the Total Active B-8 Solar Neutrino Flux Using an Array of He-3 Proportional Counters at the Sudbury Neutrino Observatory, SNO Collaboration, Phys. Rev. Lett. 101, 111301 (2008).

6. Wavelength Shifters for Water Cherenkov Detectors, X. Dai et al., Nucl. Instrum. Meth. 589, 290 (2008).

7. Measurement of the Cosmic Ray and Neutrino-Induced Muon Flux at the Sudbury Neutrino Observatory, SNO Collaboration, Phys. Rev. D80, 012001 (2009).

8. A Simple radionuclide-driven single-ion source, M. Montero Diez et al., Rev. Sci. Instrum. 81, 113301 (2010). 


\section{Contents}

Abstract ...................... ii

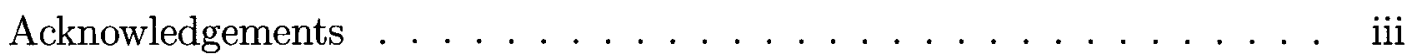

Statement of Originality . . . . . . . . . . . iv

List of Tables viii

$\begin{array}{ll}\text { List of Figures } & \text { ix }\end{array}$

1 Introduction 1

2 Neutrinos 4

2.1 Neutrino Oscillations . . . . . . . . . . . . . . . . 5

2.1 .1 Survival Probability . . . . . . . . . . . . 8

2.1 .2 Mass Hierarchy . . . . . . . . . . . . . . . . . 8

2.2 Experimental mass measurements . . . . . . . . . . . . . 9

$2.2 .1 \quad \beta$ decay $\ldots \ldots \ldots \ldots \ldots$

2.2 .2 Astrophysics . . . . . . . . . . . . . . . . 10

2.3 Double beta decay . . . . . . . . . . . . . . . . 11

$2.3 .10 \nu \beta \beta$ Neutrino Mass Measurement . . . . . . . . . . 13

2.3 .2 Other physics . . . . . . . . . . . . . . . 20

3 Enriched Xenon Observatory 27

3.1 Liquid Xenon Prototype . . . . . . . . . . . . . . . . . . . . 29

3.2 Gas Xenon Prototype . . . . . . . . . . . . . . . . . . . 31

3.2 .1 Track topology . . . . . . . . . . . . . . 32

4 Barium Ion Laser Induced Fluorescence $\quad 44$

4.1 Laser Induced Fluorescence Theory . . . . . . . . . . . . . . . . 45

4.1 .1 Ionic Species of Barium . . . . . . . . . . . . 45

4.1 .2 Energy Levels of $\mathrm{Ba}^{+} \ldots \ldots \ldots \ldots$ 
4.1.3 Different Procedures Investigated . . . . . . . . . . 47

4.1.4 Classical Ion-Atom Collision Theory . . . . . . . . . . 52

4.1 .5 Quenching Ratio . . . . . . . . . . . . . . . . 57

4.1 .6 Linewidth . . . . . . . . . . . . . . . . . 58

4.2 Laser Induced Fluorescence Apparatus . . . . . . . . . . . . . . 61

4.3 Electrical Discharge Ion Source . . . . . . . . . . . . . . . 62

4.3.1 Barium Fluorescence Data from Electric Discharges . . . . . 66

4.4 Laser Ablation Ion Source . . . . . . . . . . . . . . . . . . . 72

4.4.1 Laser Beam Size and Intensity . . . . . . . . . . . . 75

4.4 .2 Ablation Rate Decay . . . . . . . . . . . . . . 75

4.5 Time of Flight of Ions from Laser Ablation . . . . . . . . . . 77

4.6 Time of Flight Theory . . . . . . . . . . . . . . . 78

4.6 .1 Ion Energy Spectrum . . . . . . . . . . . . . . 80

4.6.2 Charge Density Effect . . . . . . . . . . . . . . 81

4.6.3 Barium Mass Spectrum Analysis _. . . . . . . . . . 83

4.6.4 Chamber Drift Constant . . . . . . . . . . . . . . . . . 83

4.7 Laser Ablation Ion Fluorescence . . . . . . . . . . . . . . . 87

4.7 .1 Ion Drift . . . . . . . . . . . . . . . . . 92

4.7.2 Linewidth and Position . . . . . . . . . . . . . . . 93

5 Barium Extraction and Conversion $\quad 96$

5.1 Triple Quadrupole Mass Spectrometer . . . . . . . . . . . . 97

5.1 .1 Electrospray Ion Source . . . . . . . . . . . . . . . . . 99

5.1 .2 Quadrupole Ion Guide . . . . . . . . . . . . . . . . . 104

5.1 .3 Collision Cell . . . . . . . . . . . . . . . . . . . . . 113

5.1.4 Mass Spectrometer Operation Methods . . . . . . . . . . . 113

5.1.5 Mass Spectrometer Calibration . . . . . . . . . . . . . 114

5.2 Charge Transfer Probability . . . . . . . . . . . . . . . . . 114

6 Conclusion 121

A Analysis of the hep neutrino flux $\quad 124$

A.1 Systematic uncertainties . . . . . . . . . . . . . . . . . 129

A.1.1 Energy resolution and energy scale . . . . . . . . . . 129

A.1.2 Oscillation parameters . . . . . . . . . . . . . . 129

A.1.3 Neutrino cross section . . . . . . . . . . . . . 130

A.1.4 Other uncertainties . . . . . . . . . . . . . . . 133

A.2 Results . . . . . . . . . . . . . . . . . . . . 134

$\begin{array}{ll}\text { References } & 136\end{array}$ 


\section{List of Tables}

2.1 Neutrino mixing angle experimental measurements $\ldots \ldots \ldots$

$2.2 \Delta m^{2}$ experimental measurements . . . . . . . . . . . 7

2.3 List of $0 \nu \beta \beta$ isotopes with their energy endpoints and their natural abundances . . . . . . . . . . . . . . . . 12

2.4 Calculated values of $T_{1 / 2}^{0 \nu \beta \beta}$ for ${ }^{136} \mathrm{Xe} \ldots \ldots \ldots \ldots \ldots$

2.5 Calculated values of $T_{1 / 2}^{2 \nu \beta \beta}$ for ${ }^{136} \mathrm{Xe} \ldots \ldots \ldots \ldots \ldots$

2.6 Current experimental limits on $T_{1 / 2}^{0 \nu \beta \beta} \ldots \ldots \ldots \ldots \ldots 20$

2.7 Value of the parameters used in the angular correlation analysis . . 25

4.1 List of broadening causes of the $P_{1 / 2}-S_{1 / 2}$ transition $\ldots \ldots \ldots 71$

5.1 Parameters of the electrospray source and mass spectrometer . . . 102

5.2 Barium isotope content of the electrospray source . . . . . . . . 104

5.3 Peaks used for the calibration of the mass spectrometer . . . . . . 116

5.4 List of peaks measured after the instrument calibration . . . . . 116

A.1 Description of the two reactions producing neutrinos above the SNO energy threshold . . . . . . . . . . . . . 126 


\section{List of Figures}

2.1 Standard Model of Particle Physics . . . . . . . . . . . . 6

2.2 Mass spectrum of fundamental particles of the Standard Model . . 6

2.3 Different mass hierarchies possible for neutrinos . . . . . . . . 9

$2.4{ }^{136}$ Xe decay scheme and energy levels . . . . . . . . . . . . 13

2.5 Tree-level Feynman diagrams for the $2 \nu \beta \beta$ and the $0 \nu \beta \beta$ decays . . 14

2.6 Energy spectrum of the $0 \nu \beta \beta$ and $2 \nu \beta \beta$ decays . . . . . . . 17

2.7 Energy spectrum of the $0 \nu \beta \beta$ and $2 \nu \beta \beta$ decays enlarged near the $0 \nu \beta \beta$ decay energy ................... 18

2.8 Allowed region from neutrino oscillation and cosmology experiments of the neutrino effective mass and lowest mass plane . . . . . . . 19

2.9 Generic diagram for $0 \nu \beta \beta$ decay . . . . . . . . . . . . . 22

2.10 Rearranged $0 \nu \beta \beta$ diagram to show the Majorana nature of the neu-

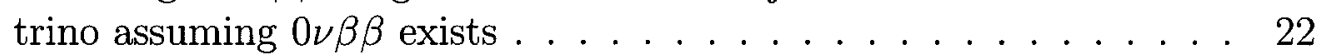

2.11 Probability density function of the electron angular correlation distribution for $0 \nu \beta \beta$ decays . . . . . . . . . . . . . . 23

2.12 Projection of the expected number of years required to reach the LEP limit on the $W_{R}$ mass $\ldots \ldots \ldots \ldots \ldots \ldots$

3.1 Schematics of the EXO-200 experiment . . . . . . . . . . 30

3.2 Schematics of the EXO-200 TPC . . . . . . . . . . 30

3.3 Schematics of the XEP chamber . . . . . . . . . . . . . 32

3.4 A typical electron track in xenon . . . . . . . . . . . 34

3.5 Maximum track distance from the origin distribution . . . . . . . 35

3.6 Average maximum distance of tracks as a function of pressure . . . 37

3.7 Track density and reconstructed energy for $1 \mathrm{MeV}$ electrons in $10 \mathrm{~atm}$ xenon . . . . . . . . . . . . . . 38

3.8 Fraction of events having $99 \%$ of their energy contained as a function

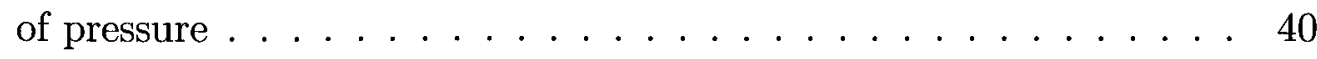


3.9 Track density and reconstructed energy for $1 \mathrm{MeV}$ electrons in $10 \mathrm{~atm}$ xenon with an improved source . . . . . . . . . . . 41

3.10 Number of large angle collisions as a function of pressure . . . . . . 43

3.11 Fraction of events with a bremsstrahlung gamma as a function of

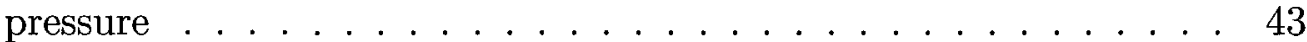

$4.1 \mathrm{Ba}^{+}$energy levels . . . . . . . . . . . . . . . . 47

$4.2 \mathrm{Ba}^{+}$and $\mathrm{Ar}$ potential shape $\ldots \ldots \ldots \ldots \ldots \ldots$

4.3 Collision cross-section schematic . . . . . . . . . . . . . 56

4.4 Calculated quenching rate of barium ions in noble gases . . . . . . . 58

4.5 Absorption and fluorescence spectra of the Coumarin 480 and DCM Special dyes . . . . . . . . . . . . . . . . . 6 63

4.6 Dye laser spectra . . . . . . . . . . . . . . . . . . . . . 64

4.7 Arcing chamber schematic . . . . . . . . . . . . . 65

4.8 Emission spectrum of the arc using barium metal as a source . . . . 66

4.9 Photographs of the LIF of $\mathrm{Ba}^{+}$ions generated by the electric discharge ion source . . . . . . . . . . . . . . . 68

4.10 Braching fraction of the $P_{1 / 2}$ state decay to the $S_{1 / 2}$ and $D_{3 / 2}$ states 69

4.11 Line profile of the $S_{1 / 2}-P_{1 / 2}$ transition at 30 mbar of argon . . . 71

4.12 Target damage traces observed through a microscope . . . . . . 76

4.13 Decay of the ion signal with time . . . . . . . . . . . 76

4.14 Copper mount for the barium target . . . . . . . . . . . . . 77

4.15 ToF chamber schematic . . . . . . . . . . . . . . . . . 79

4.16 Simulation of ion paths through a cut of the ToF chamber . . . . 79

4.17 Time of flight of $\mathrm{Ba}$ ions through a model of the chamber as a function of their kinetic energy _. . . . . . . . . . 80

4.18 Kinetic energy spectrum from laser ablation . . . . . . . . . . . 82

4.19 Simulated ToF spectra showing the effect of interactions in the ion cloud on the ToF spectrum resolution . . . . . . . . . . . . 84

4.20 Mean and RMS of the ToF distribution as a function of the number of ions in the cloud . . . . . . . . . . . . . . . 84

4.21 Mass spectrum from laser ablation of a pure Ba target $\ldots . . . .85$

4.22 Barium line position as a function of the drift voltage . . . . . . 86

4.23 Charge collection intensity spectrum for a typical run in vacuum . . 87

4.24 Fluorescence chamber internal schematic . . . . . . . . . . . . . 89

4.25 Average trace recorded of ions emitted from laser ablation . . . . . 90

4.26 Fluorescence time spectrum. . . . . . . . . . . . . . . . . 91

4.27 Correlation between blue laser intensity and background counting rates . . . . . . . . . . . . . . . . . . . 94

4.28 Line profile of the $S_{1 / 2}-P_{1 / 2}$ transition in vacuum $\ldots \ldots \ldots 95$ 
5.1 Mass spectrometer schematic $\ldots \ldots \ldots \ldots$

5.2 Electrospray source schematic . . . . . . . . . . . . . . 100

5.3 Typical barium acetate mass spectrum from the electrospray source 101

5.4 Electrospray source spectrum with optimized parameters . . . . . 103

5.5 SIMION simulations of a $100 \mathrm{Da}$ ion trough a $50 \mathrm{~mm}$ quadrupole. . 105

5.6 SIMION simulations of a $120 \mathrm{Da}$ ion trough a $50 \mathrm{~mm}$ quadrupole . 105

5.7 Mathieu function stability regions . . . . . . . . . . . . 108

5.8 Mathieu function stability regions for the Quattro II mass spectrometers . . . . . . . . . . . . . . . . . . . . 109

5.9 FWHM of the $\mathrm{Ba}^{++}$peak for different LM Res and HM Res values 111

5.10 Intensity of the $\mathrm{Ba}^{++}$peak for different LM Res and HM Res values 112

5.11 Fragmentation mass spectra . . . . . . . . . . . . 115

5.12 Examples of charge exchange spectra using TEA as the collision gas 118

5.13 Conversion probability as a function of TEA pressure and collision energy . . . . . . . . . . . . . . . . . . . . 119

A.1 Spectra for ${ }^{8} \mathrm{~B}$ and hep neutrinos $\ldots \ldots \ldots \ldots \ldots \ldots$

A.2 Radial distribution of the production of ${ }^{8} \mathrm{~B}$ and hep neutrinos in the Sun . . . . . . . . . . . . . . . . . . 127

A.3 Simulated event number as a function of energy . . . . . . . . 128

A.4 Oscillation parameter sampling used to extract survival probabilities shown in Figure A.5 . . . . . . . . . . . . . . . . 130

A.5 Variation of the survival probability for ${ }^{8} \mathrm{~B}$ and hep neutrinos . . . 131

A.6 Root mean square value of the survival probability as a function of energy, sampling the allowed region in the oscillation plane for both hep and ${ }^{8} \mathrm{~B}$ neutrinos . . . . . . . . . . . . . . . . . . 132

A.7 hep data and background estimation spectra . . . . . . . . 135 


\section{Chapter 1}

\section{Introduction}

Neutrinos are a major component of the Universe. Learning about them is an essential endeavour to understand the subatomic world, but also the large scale structure of the Universe. They have been studied for the last 70 years with many great successes despite their very weak interaction with matter. One of neutrino's fundamental properties that remains a mystery is their mass. Scientists invest time and resources to measure it and understand the underlying mechanism that allows neutrinos to have a mass. The measurement of neutrinoless double beta decay $(0 \nu \beta \beta)$ is a natural continuation of the quest to find out more vital information about neutrinos which would lead to a better understanding of many aspects of the Standard Model of particle physics (SM), with its extensions, and the dynamics of the Universe.

The Enriched Xenon Observatory (EXO) is one of many experiments trying to measure the $0 \nu \beta \beta$ decay rate. The EXO collaboration counts more than $60 \mathrm{mem}$ bers based at 13 different institutions spread around the world. It is a collaboration that grows quickly and although the main effort is directed toward research and development, physics results are expected in the near future. The EXO experiment is unique in many ways, but its main advantage is the possibility of tagging the daughter of the reaction, eliminating background events coming from natural and 
cosmogenic radioactivity. Any future observation claims of $0 \nu \beta \beta$ decay will require a strong understanding of background events in the vicinity of the signal. Tagging the daughter of the reaction, in this case a barium ion, is performed by exciting the ion with a laser and then observing photons created by its de-excitation. The transition occurs at a very specific energy and can be considered as a fingerprint of the element. This technique is very powerful. It is sensitive at the single atom level and discriminates against any decay that does not produce a barium ion.

The concept of barium tagging is still being investigated and questions about the technique need to be answered. Does the ion need to be extracted from the xenon volume and is there a way to do it efficiently? Is it possible to convert efficiently the doubly charged barium ion into the singly charged one required for tagging? Is the barium ion stable in gas or does it form molecules? What is the drift velocity of ions in xenon? This thesis will answer some of these questions. It is divided in four main chapters.

Chapter 2 describes the current knowledge about neutrinos and what information the neutrinoless double beta decay can unveil about neutrinos. It lists and explains the different experimental techniques that allow for a neutrino mass measurement. It concludes on an analysis performed on the angular resolution of the two electron tracks of the decay required to probe a new type of physics.

Chapter 3 describes two EXO prototypes built to perform the energy measurement of the decay. The first one, EXO-200, uses xenon in its liquid phase while the second one, XEP, is a gas phase experiment. Simulated ionizing track topologies in gas will be quickly described. Finally, detailed work carried out to specify the physical size of the prototype will be explained.

Chapter 4 relates the efforts made in tagging barium in gas. It describes two barium ion sources. The first one uses electrical discharges between two electrodes in low pressure gas. The second one uses a pulsed ultraviolet laser focused on a solid target. The characterization of each ion source is also included. This chapter also lists some of the spectroscopic measurements of the barium ion transitions 
probed during the study.

Chapter 5 answers a fundamental question related to barium tagging. It outlines measurements that prove that the doubly charged barium ion can acquire an electron from a donor gas without forming a molecule and shows that $\mathrm{Ba}^{++}$might be more stable that previously thought. It also describes the instrument used to perform the measurements.

The Appendix describes the analysis of some of the Sudbury Neutrino Observatory data as an attempt to measure the hep neutrino flux from the Sun. Taking into account the uncertainty in the ${ }^{8} \mathrm{~B}$ flux and in the neutrino oscillation parameters, an upper limit on the flux has been calculated. 


\section{Chapter 2}

\section{Neutrinos}

The Standard Model of Particle Physics (SM) describes fundamental properties of subatomic particles. Figure 2.1 shows the particle content of the model. Only a few pieces are missing from the puzzle and many of them are related to neutrinos. These subatomic particles are unique in many ways. They are the only chargeless fermions, not interacting via the electromagnetic or the strong force, and thus making them very precise probes to measure the weak force fundamental constants. The SM requires them to have no mass while experiments have shown the contrary, opening a window for physics beyond the SM. They might be the only particles having a Majorana mass term, which is forbidden to the other fermions, explaining the very different mass scale of neutrinos compared to other fundamental particles shown in Figure 2.2. The $0 \nu \beta \beta$ decay also violates the lepton number conservation by two units. The observation of the decay would be an enormous discovery, especially given its ability to explain the asymmetry between matter and antimatter, as well as to understand the first moments of the Universe after the Big Bang. The physics community has identified the neutrinoless double beta $(0 \nu \beta \beta)$ decay reaction as an excellent mechanism to answer the questions above. The simple existence of $0 \nu \beta \beta$ decay would prove that neutrinos are Majorana particles and that lepton number is not always conserved, while the decay rate would 
measure the neutrino mass.

This chapter introduces different neutrino characteristics and describes the techniques used to study them. Section 2.1 explains neutrino oscillations and how it solved fundamental problems of particle physics and astrophysics. Section 2.2 describes the many ways scientists are trying to measure the neutrino mass. Section 2.3 describes the core of this thesis, the measurement of the $0 \nu \beta \beta$ decay rate and its implications for the SM. Finally, Section 2.3.2 presents some phenomenological work on a $0 \nu \beta \beta$ model, adapted and extended exclusively for this thesis to justify a tracking detector for a $0 \nu \beta \beta$ measurement.

\section{$2.1 \quad$ Neutrino Oscillations}

The first hint of a non-zero neutrino mass was observed with the Ray Davis experiment [2] in the early 1970's when it measured a shortage of electron neutrinos coming from the Sun. At the time, few believed that the low rate was due to the neutrino mass. The first proof that neutrinos had a non-zero mass was unveiled 25 years later, when Super-Kamiokande [3] measured oscillations of atmospheric neutrinos. Then, a series of experiments (SNO, KamLAND, LSND, OPERA, ...) [4-7] confirmed the observation with neutrinos from the Earth's atmosphere, the Sun, nuclear reactors and particle accelerators.

Neutrino oscillations is due to a difference between the neutrino flavour eigenstates and their mass eigenstates. The flavour that is measured in an experiment is in fact a combination of the three mass eigenstates and the relationship between them is parametrized by the Pontecorvo-Maki-Nakagawa-Sakata (PMNS) [8] lepton mixing matrix:

$$
U=\left(\begin{array}{ccc}
U_{e 1} & U_{e 2} & U_{e 3} \\
U_{\mu 1} & U_{\mu 2} & U_{\mu 3} \\
U_{\tau 1} & U_{\tau 2} & U_{\tau 3}
\end{array}\right)
$$




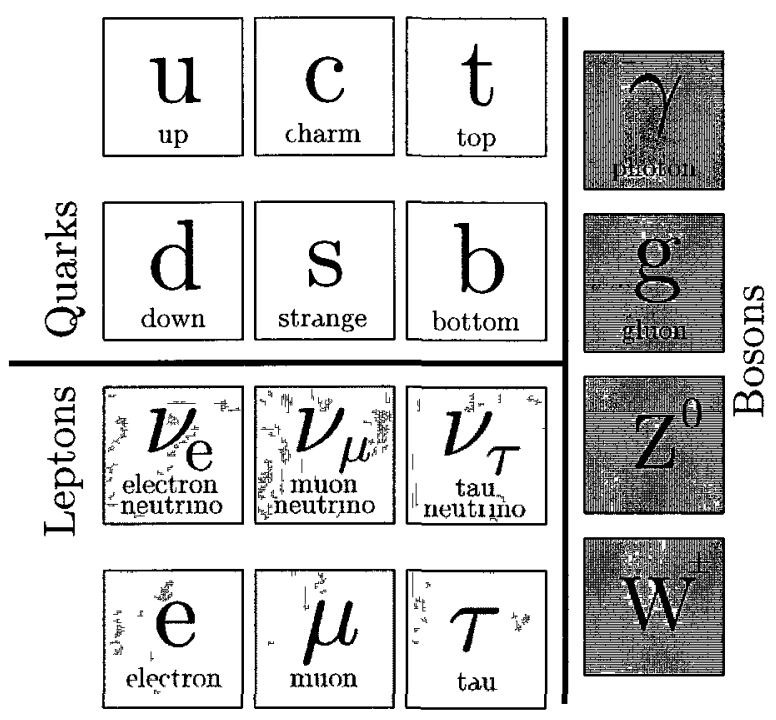

Figure 2.1: Standard Model of Particle Physics content and details about fundamental particles.

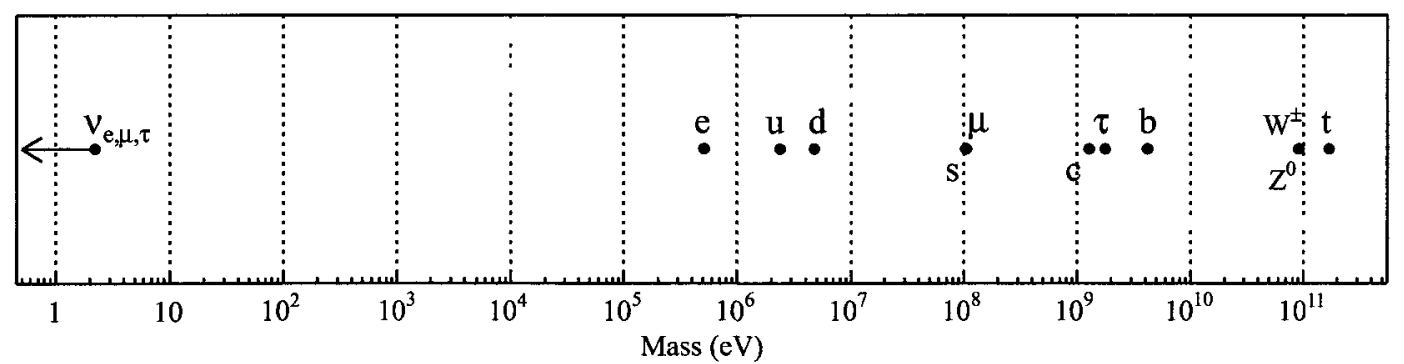

Figure 2.2: Mass spectrum of fundamental particles of the Standard Model. Note the logarithmic mass scale and the very low mass of neutrinos. Mass values are taken from the Particle Data Group (PDG) review [1]. 


$$
U=\left(\begin{array}{ccc}
c_{12} c_{13} e^{\imath \alpha_{1} / 2} & s_{12} c_{13} & s_{13} e^{-\imath \delta} \\
-s_{12} c_{23}-c_{12} s_{23} s_{13} e^{\imath \delta} & \left(c_{12} c_{23}-s_{12} s_{23} s_{13} e^{\imath \delta}\right) e^{\imath \alpha_{2} / 2} & s_{23} c_{13} \\
s_{12} s_{23}-c_{12} c_{23} e^{\imath \delta} & -c_{12} s_{23}-s_{12} c_{23} s_{13} e^{\imath \delta} & c_{23} c_{13}
\end{array}\right)
$$

In these matrices, $c_{\imath \jmath}$ means $\cos \theta_{\imath \jmath}, s_{\imath \jmath}$ means $\sin \theta_{\imath \jmath}, \delta$ is the $\mathrm{CP}$ violating phase, and $\alpha_{\imath}$ are the two Majorana phases that exist only if neutrinos have a Majorana nature. Each $\theta_{\imath \jmath}$ represents the mixing angle between two of the three neutrino flavours. The current experimental measurements of the angles are listed in Table 2.1.

The mixing angles are not the only parameters involved in the neutrino oscillations. The other parameters are the differences between the mass squared of the eigenstates $\left(\Delta m_{\imath \jmath}^{2}=m_{\imath}^{2}-m_{\jmath}^{2}\right)$. There are three $\Delta m_{\imath \jmath}^{2}$ for three neutrino flavours, but only two are independent. The current experimental measurements of the mass differences are listed in Table 2.2 .

\begin{tabular}{|c|c|c|c|}
\hline Parameter & $\begin{array}{c}\text { Experimental } \\
\text { measurement }\end{array}$ & $\begin{array}{c}\text { Confidence } \\
\text { Level (\%) }\end{array}$ & Reference \\
\hline \hline $\sin ^{2}\left(2 \theta_{12}\right)$ & $0.86_{-004}^{+003}$ & 68 & KamLAND + solar [9] \\
\hline $\sin ^{2}\left(2 \theta_{23}\right)$ & $>0.92$ & 90 & Super-Kamiokande [10] \\
\hline $\sin ^{2}\left(2 \theta_{13}\right)$ & $<0.19$ & 90 & CHOOZ [11] \\
\hline
\end{tabular}

Table 2.1: Neutrino mixing angle experimental measurements.

\begin{tabular}{|c|c|c|c|}
\hline Parameter & $\begin{array}{c}\text { Experimental measurement } \\
\left(\mathrm{eV}^{2}\right)\end{array}$ & $\begin{array}{c}\text { Confidence } \\
\text { Level (\%) }\end{array}$ & Reference \\
\hline \hline$\Delta m_{21}^{2}$ & $8.0_{-03}^{+04} \times 10^{-5}$ & 68 & KamLAND + solar [9] \\
\hline$\Delta m_{32}^{2}$ & $\pm 2.4_{-05}^{+06} \times 10^{-3}$ & 68 & MINOS [12] \\
\hline$\Delta m_{31}^{2}$ & $=\Delta m_{32}^{2}+\Delta m_{21}^{2} \simeq \Delta m_{32}^{2}$ & - & - \\
\hline
\end{tabular}

Table 2.2: $\Delta m^{2}$ experimental measurements. Note that the sign of $\Delta \mathrm{m}_{32}^{2}$ is not known. 


\subsubsection{Survival Probability}

A useful quantity when dealing with oscillations is the survival probability. For oscillations in vacuum, assuming only two neutrino flavours, the survival probability is given by:

$$
P_{\alpha \alpha}=1-\sin ^{2} 2 \theta \sin ^{2}\left(1.27 \frac{L}{E} \Delta m^{2}\right)
$$

where $\mathrm{P}_{\alpha \alpha}$ is the probability of a flavour $\alpha$ neutrino of staying in the $\alpha$ flavour, $L$ is the propagation distance in $\mathrm{km}, E$ is the neutrino energy in $\mathrm{GeV}, \Delta m^{2}$ is the mass squared difference between the two eigenstates in $\mathrm{eV}^{2}$ and $\theta$ the mixing angle between those two states. Therefore, knowing the number of neutrinos of each flavour generated at the source and the number reaching the detector, it is possible to measure $\theta$ and $\Delta m^{2}$.

\subsubsection{Mass Hierarchy}

The sign of $\Delta m_{32}^{2}$ is unknown making it impossible to know the hierarchy of neutrino masses. If the mass eigenstate $m_{1}$, which is the main part of $\nu_{e}$, is the lightest mass eigenstate, then it is a normal hierarchy. The other possibility is to have $m_{3}$, which has almost no $\nu_{e}$ component, as the lightest eigenstate. Then it is called an inverted hierarchy because of the inverse ordering compared to the charged lepton hierarchy. If the lightest eigenstate is heavy $(>0.1 \mathrm{eV})$, then the mass difference between states is negligible compared to their total mass. It is then called a degenerate hierarchy. Figure 2.3 shows the two mass orders with the flavour components of each eigenstate. A drawback of measuring the difference of masses is the missing mass scale. The scientific community has to rely on other techniques to know the absolute mass scale. 


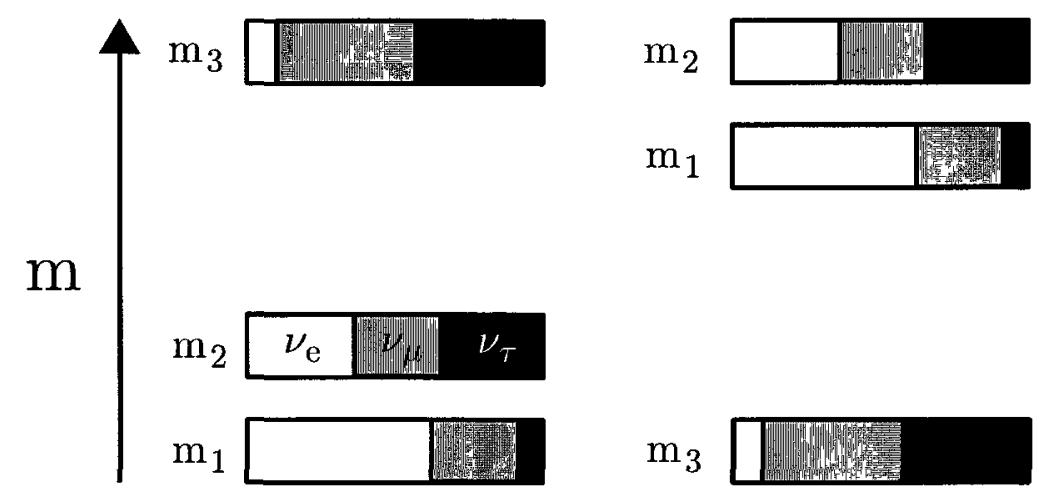

Figure 2.3: The normal mass hierarchy is depicted on the left and the inverted one is on the right. The white section of the bar corresponds to the electron flavour proportion of the mass eigenstate, the gray corresponds to the muon flavour and the black, the tau flavour. Proportions are approximate.

\subsection{Experimental mass measurements}

\subsection{1 $\beta$ decay}

In principle, the simplest way to measure the neutrino mass is to look at the energy of the electron emitted by a $\beta$ decay, on what is called a Kurie plot. By looking at the highest energy events and comparing with the total energy available one can extract the electron neutrino mass by considering the neutrino emitted at rest. In reality, this measurement is very challenging due to the small mass of the neutrino. Most of the $\beta$ decay experiments use tritium as the source, which has an energy output of $18570 \mathrm{eV}$ compared to a missing energy smaller than $1 \mathrm{eV}$.

The upper limit for the electron neutrino mass extracted from the Troitsk [13] and Mainz [14] experiments were $2.5 \mathrm{eV}$ and $2.3 \mathrm{eV}$ respectively. The Katrin experiment is expected to achieve an upper limit of $0.2 \mathrm{eV}$ in the near future. It is interesting to note that the best fitted values for the electron neutrino mass squared $m_{\nu_{e}}^{2}$ from $\beta$ decay experiments have all been smaller than zero. It has been recognized that this was probably due to systematic errors. 


\subsubsection{Astrophysics}

\section{Cosmology}

The large scale structure of the Universe brings very important information about the neutrino mass. The shape of the Universe provides a measurement of its total mass. Since neutrinos are the second most common particle in the Universe after photons, a tiny neutrino mass would produce a measurable effect on a large scale. Then, one can look at the distribution of galaxies in the Universe and the cosmic microwave background (CMB) and extract an upper limit for the sum of all neutrino flavour masses $\left(\sum_{\imath=0}^{n} m_{\imath}\right)$. Using the Wilkinson Microwave Anisotropy Probe (WMAP) and the 2dFGRS data [15] the upper limit was established at $\sum_{\imath=0}^{n} m_{\imath}<0.63 \mathrm{eV}$. The CMB probe called Planck used in conjunction with the Sloan Digital Sky Survey (SDSS) should reduce the limit to $0.1 \mathrm{eV}$. Unfortunately, the measurement involves many astrophysics models and might not be as convincing as a $\beta$ decay experiment. Nevertheless, the cosmology measurement involves all neutrino flavours which would complement a measurement from beta decay.

\section{Supernova}

During a supernova explosion the largest portion of the energy is carried away by neutrinos. The kinetics of massive particles tells us that for the same kinetic energy the lightest particles travel faster. Therefore, on an astronomic scale, a small mass difference results in a measurable arrival time difference:

$$
\Delta t=0.0514 D\left(m_{\nu} / E\right)^{2}
$$

where $\Delta t$ is the time delay in seconds with respect to the arrival time of a massless particle, $D$ is the distance travelled in kpc, $E$ the energy of the neutrino in $\mathrm{MeV}$ and $m_{\nu}$ the mass of the neutrino in $\mathrm{eV}$. 
Unfortunately, supernova explosion are very complex and models are primitive. Furthermore, the explosion rate of supernovae in our galaxy is very low, about 1 per $50 \mathrm{yr}$. The only supernova for which neutrinos have been detected happened in 1987. Three detectors (Kamiokande II, IMB and Baksan) detected a total of 29 candidate events from supernova SN1987A from which an upper limit of $5.7 \mathrm{eV}$ for the electron neutrino mass has been recently extracted at a confidence level of 95\% [16], The limit found is not as sensitive as other experiments, but the technique is an independent one. A large improvement will be possible with the larger and more sensitive neutrino detectors under construction measuring neutrinos from nearby supernovae.

\subsection{Double beta decay}

Single $\beta$ decay is the transformation of an up quark into a down quark with the emission of an electron and a $\overline{\nu_{e}}$ via the weak interaction. Double beta decay $(\beta \beta)$ is simply two $\beta$ decays occurring at the same time. It has been postulated by M. Goeppert-Mayer in 1935 [17] and has been observed in more than ten isotopes. The lifetime of the reaction is of the order of $10^{18}$ to $10^{24} \mathrm{yr}$. The $\beta \beta$ reaction occurs naturally for every isotope where two successive $\beta$ decays exist. Unfortunately, in most cases it is impossible to differentiate between two coincident $\beta$ decay events that occurs in the same region and the $\beta \beta$ decay. For some isotopes, the first $\beta$ decay energetically is forbidden but allows the $\beta \beta$ decay. The ${ }^{136} \mathrm{Xe}$ atom is an example of this and its decay scheme and energy levels are shown in Figure 2.4. The ${ }^{136} \mathrm{Xe}$ to ${ }^{136} \mathrm{Cs} \beta$ decay is energetically forbidden but the $\beta \beta$ decay to ${ }^{136} \mathrm{Ba}$ is possible. Table 2.3 lists properties of the most common $\beta \beta$ decay isotopes.

If neutrinos are their own antiparticles and if they have a mass, a special phenomenon occurs and $0 \nu \beta \beta$ decay could be observed. In the $0 \nu \beta \beta$ decay, the neutrino created from one $\beta$ decay changes helicity and is absorbed at the second $\beta$ decay vertex. No neutrinos are emitted, it is a virtual exchange. Figure 2.5 


\begin{tabular}{|c|c|c|}
\hline Reaction & Energy endpoint (keV) & Natural abundance (\%) \\
\hline \hline${ }^{46} \mathrm{Ca} \rightarrow{ }^{46} \mathrm{Ti}$ & $990.4 \pm 2.4$ & 0.004 \\
\hline${ }^{70} \mathrm{Zn} \rightarrow{ }^{70} \mathrm{Ge}$ & $1000.9 \pm 3.4$ & 0.6 \\
\hline${ }^{76} \mathrm{Ge} \rightarrow{ }^{76} \mathrm{Se}$ & $2039.006 \pm 0.050$ & 7.44 \\
\hline${ }^{82} \mathrm{Se} \rightarrow{ }^{82} \mathrm{Kr}$ & $2995.1 \pm 2.0$ & 8.73 \\
\hline${ }^{86} \mathrm{Kr} \rightarrow{ }^{86} \mathrm{Sr}$ & $1255.6 \pm 2.4$ & 17.3 \\
\hline${ }^{94} \mathrm{Zr} \rightarrow{ }^{94} \mathrm{Mo}$ & $1144.1 \pm 2.0$ & 17.38 \\
\hline${ }^{100} \mathrm{Mo} \rightarrow{ }^{100} \mathrm{Ru}$ & $3034 \pm 6$ & 9.63 \\
\hline${ }^{104} \mathrm{Ru} \rightarrow{ }^{104} \mathrm{Pd}$ & $1300 \pm 4$ & 18.7 \\
\hline${ }^{110} \mathrm{Pd} \rightarrow{ }^{110} \mathrm{Cd}$ & $2000 \pm 11$ & 11.72 \\
\hline${ }^{114} \mathrm{Cd} \rightarrow{ }^{114} \mathrm{Sn}$ & $536.8 \pm 3.3$ & 28.73 \\
\hline${ }^{116} \mathrm{Cd} \rightarrow{ }^{116} \mathrm{Sn}$ & $2805.0 \pm 3.8$ & 7.49 \\
\hline${ }^{124} \mathrm{Sn} \rightarrow{ }^{124} \mathrm{Te}$ & $2287.0 \pm 1.5$ & 5.79 \\
\hline${ }^{128} \mathrm{Te} \rightarrow{ }^{128} \mathrm{Xe}$ & $867.2 \pm 1.0$ & 31.69 \\
\hline${ }^{130} \mathrm{Te} \rightarrow{ }^{130} \mathrm{Xe}$ & $2528.8 \pm 1.3$ & 33.80 \\
\hline${ }^{134} \mathrm{Xe} \rightarrow{ }^{134} \mathrm{Ba}$ & $830.1 \pm 3.0$ & 10.4 \\
\hline${ }^{136} \mathrm{Xe} \rightarrow{ }^{136} \mathrm{Ba}$ & $2468 \pm 7$ & 8.9 \\
\hline${ }^{142} \mathrm{Ce} \rightarrow{ }^{142} \mathrm{Nd}$ & $1416.9 \pm 2.2$ & 11.08 \\
\hline${ }^{148} \mathrm{Nd} \rightarrow{ }^{148} \mathrm{Sm}$ & $1928.9 \pm 1.9$ & 5.76 \\
\hline${ }^{150} \mathrm{Nd} \rightarrow{ }^{150} \mathrm{Sm}$ & $3367.5 \pm 2.2$ & 5.64 \\
\hline${ }^{154} \mathrm{Sm} \rightarrow{ }^{154} \mathrm{Gd}$ & $1251.0 \pm 1.3$ & 22.7 \\
\hline${ }^{160} \mathrm{Gd} \rightarrow{ }^{160} \mathrm{Dy}$ & $1729.7 \pm 1.3$ & 21.86 \\
\hline${ }^{170} \mathrm{Er} \rightarrow{ }^{170} \mathrm{Yb}$ & $653.6 \pm 1.7$ & 14.9 \\
\hline${ }^{176} \mathrm{Yb} \rightarrow{ }^{176} \mathrm{Hf}$ & $1086.7 \pm 1.9$ & 12.7 \\
\hline${ }^{198} \mathrm{Pt} \rightarrow{ }^{198} \mathrm{Hg}$ & $1047 \pm 3$ & 7.2 \\
\hline
\end{tabular}

Table 2.3: List of $0 \nu \beta \beta$ isotopes with their energy endpoint and their natural abundance. The list excludes isotopes having other decay channels or an endpoint value lower than $500 \mathrm{keV}$. 


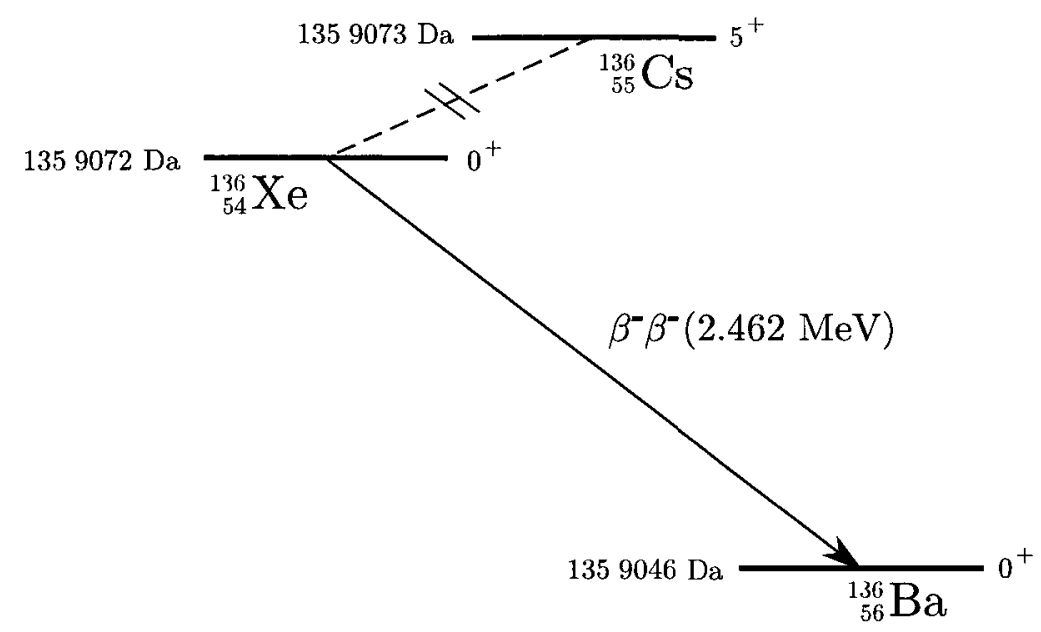

Figure 2.4: Energy levels of the ${ }^{136} \mathrm{Xe}$ and its decay scheme. The diagram shows the mass, the atomic number and the number of protons of each isotope, the nuclear spin of each state and the energy of the decay.

summarizes the two different $\beta \beta$ decay reactions. The $0 \nu \beta \beta$ decay was postulated by W.H. Furry in 1939 [18] and has not been observed yet. The standard model forbids the $0 \nu \beta \beta$ decay, but many extensions of the SM allow it. Two conditions have to be fulfilled to allow for a $0 \nu \beta \beta$ decay. The first one requires that neutrinos have a mass to allow for the helicity flip. It is now accepted that at least two of the neutrino eigenstates have a non-zero mass because of a non-zero $\Delta \mathrm{m}^{2}$, as discussed in Section 2.1. The second one requires that the neutrino is its own antiparticle. It is reasonable to think that since neutrinos are chargeless, they could be Majorana particles and their own antiparticles. If $0 \nu \beta \beta$ is possible, it would not replace the two neutrino double beta $(2 \nu \beta \beta)$ decay; both would occur.

\subsection{1 $0 \nu \beta \beta$ Neutrino Mass Measurement}

The $0 \nu \beta \beta$ decay is linked to the neutrino mass by the helicity flip. The more mass a neutrino has, the easier it would be to produce the change in helicity. Therefore, a measurement of the $0 \nu \beta \beta$ decay rate is also a measurement of the 


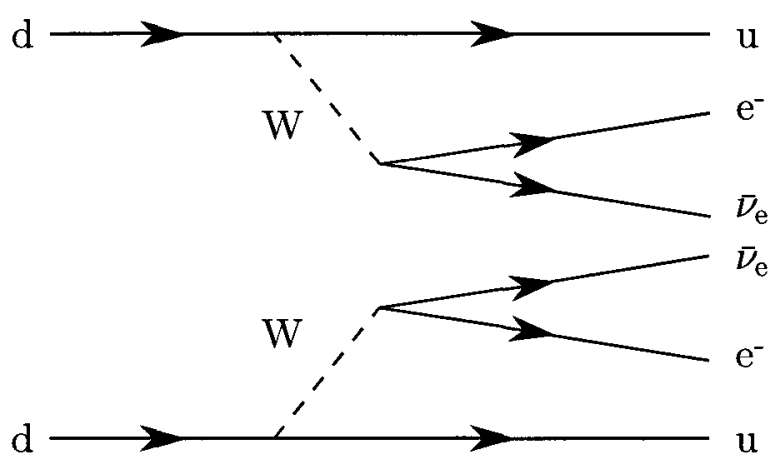

(a)

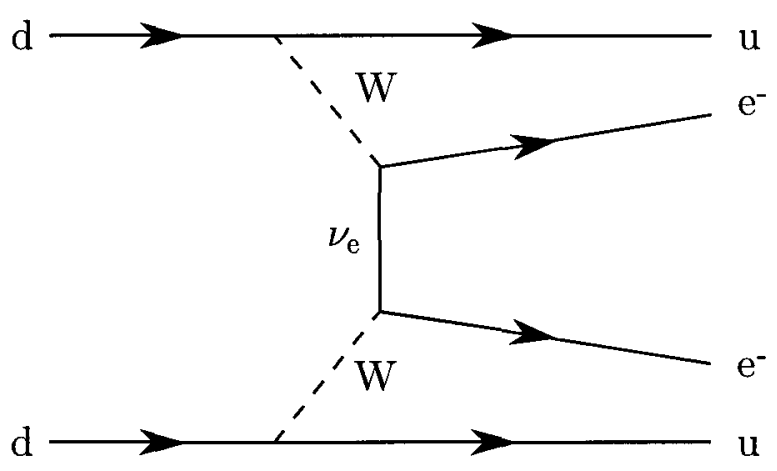

(b)

Figure 2.5: Tree-level Feynman diagrams for the $2 \nu \beta \beta$ (a) and the $0 \nu \beta \beta$ (b) decays. 


\begin{tabular}{|c|c|}
\hline Reference & $T_{12}^{0 \nu \beta \beta}(\mathrm{yr})$ \\
\hline \hline E. Caurier et al. [19] & $48.4 \times 10^{26}$ \\
\hline J. Engel et al. [20] & $13.2 \times 10^{26}$ \\
\hline A. Staudt et al. [21] & $8.8 \times 10^{26}$ \\
\hline A. Faessler et al. [22] and J. Toivanen et al. [23] & $21.2 \times 10^{26}$ \\
\hline G. Pantis et al. [24] & $7.2 \times 10^{26}$ \\
\hline
\end{tabular}

Table 2.4: Calculated values of $T_{1 / 2}^{0 \nu \beta \beta}$ for ${ }^{136} \mathrm{Xe}$ assuming $\left\langle m_{\beta \beta}\right\rangle=50 \mathrm{meV}$ for different matrix element models.

neutrino mass. While working out the amplitude of the $0 \nu \beta \beta$ decay, it is useful to define a quantity called the effective neutrino mass:

$$
\left\langle m_{\beta \beta}\right\rangle=\sum_{\imath=0}^{n} U_{e \imath}^{2} m_{\imath}
$$

The $0 \nu \beta \beta$ decay half-life $\left(T_{1 / 2}\right)$ is then given by:

$$
\left[T_{1 / 2}^{0 \nu \beta \beta}\right]^{-1}=G_{0 \nu \beta \beta}\left|M_{0 \nu \beta \beta}\right|^{2} \frac{\left\langle m_{\beta \beta}\right\rangle^{2}}{m_{e}^{2}}
$$

where $G_{0 \nu \beta \beta}$ is the phase space factor, $M_{0 \nu \beta \beta}$ is the nuclear matrix element, $\left\langle m_{\beta \beta}\right\rangle$ is the effective neutrino mass and $m_{e}$ is the electron mass. Calculation of $G_{0 \nu \beta \beta}$ are relatively accurate, but for $M_{0 \nu \beta \beta}$, calculations are more tedious and large uncertainties are associated with them. Table 2.4 summarizes some of the calculated values of $T_{1 / 2}^{0 \nu \beta \beta}$ for ${ }^{136} \mathrm{Xe}$. These values can be compared to the half-life of $2 \nu \beta \beta$, as listed in Table 2.5 .

Recently, a claim has been made by Klapdor-Kleingrothaus et al. [26] of having measured a $0 \nu \beta \beta$ half-life of $1.19_{-023}^{+037} \times 10^{25}$ years for ${ }^{76} \mathrm{Ge}$, corresponding to a mass between 0.2 and $0.6 \mathrm{eV}$ at $99.73 \%$ confidence level. Unfortunately, only a small fraction of the whole collaboration signed the paper and many people of the scientific community criticized the way the analysis was performed. The main 


\begin{tabular}{|c|c|}
\hline Reference & $T_{1 / 2}^{2 \nu \beta \beta}$ \\
\hline \hline J. Engel et al. [20] & $0.82 \times 10^{21}$ \\
\hline O. Rumyantsev and M. Urin [25] & $1.0 \times 10^{21}$ \\
\hline A. Staudt et al. [21] & $4.64 \times 10^{21}$ \\
\hline
\end{tabular}

Table 2.5: Calculated values of $T_{1 / 2}^{2 \nu \beta \beta}$ for ${ }^{136} \mathrm{Xe}$.

concern is the presence of unidentified background events making the source of the signal as uncertain as the background events. This remains the biggest challenge of any $0 \nu \beta \beta$ experiment. Current experiments will have to show that they fully understand their backgrounds if they want to perform a convincing measurement. On the other hand, many experiments measured lower limits for the half-life of the reaction as depicted in Table 2.6.

With ${ }^{136} \mathrm{Xe}$, neither the $0 \nu \beta \beta$ nor the $2 \nu \beta \beta$ decay rate have been measured. Using average values of Tables 2.4 and 2.5, the expected half-life for $0 \nu \beta \beta$ would be $2 \times 10^{27} \mathrm{yr}$, while for $2 \nu \beta \beta$ it would be $2.2 \times 10^{21} \mathrm{yr}$. The $0 \nu \beta \beta$ rate assumes a $\left\langle m_{\beta \beta}\right\rangle$ of $50 \mathrm{meV}$. Then, for 1 ton of ${ }^{136} \mathrm{Xe}\left(4.43 \times 10^{27}\right.$ atoms $)$, the decay rate would be $1.1 \mathrm{yr}^{-1}$ for $0 \nu \beta \beta$ and $1 \times 10^{6} \mathrm{yr}^{-1}$ for $2 \nu \beta \beta$.

In $0 \nu \beta \beta$ decays, the sum of the two electron energies should equal $2.479 \mathrm{MeV}$ while in $2 \nu \beta \beta$ decays the two electrons have to share the energy with the two anti-neutrinos produced. Figure 2.6 shows the energy spectrum of both types of decays for a one year exposure of one ton of ${ }^{136} \mathrm{Xe}$, using the rate calculated above, but using a $\left\langle m_{\beta \beta}\right\rangle$ of $1 \mathrm{eV}$ instead of $50 \mathrm{meV}$ to show a reasonable amount of $0 \nu \beta \beta$ events. Figure 2.7 shows similar decay spectra, but enlarged around the $0 \nu \beta \beta$ decay energy.

Even without any $0 \nu \beta \beta$ experimental results, it is possible to constrain the parameters $\left\langle m_{\beta \beta}\right\rangle$ and the value of the lowest mass eigenstate $\left(\min \left[m_{1}, m_{3}\right]\right)$ based on neutrino oscillation and cosmology data. Figure 2.8 shows the allowed region of these two parameters, for both the normal and the inverted hierarchy. Measuring the mixing angle $\theta_{13}$ would further constrain the allowed region. 


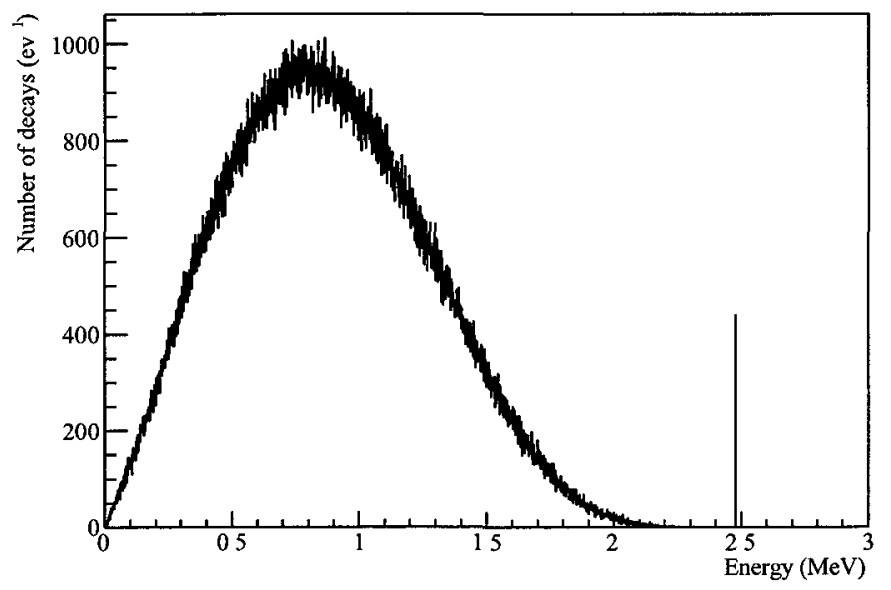

(a)

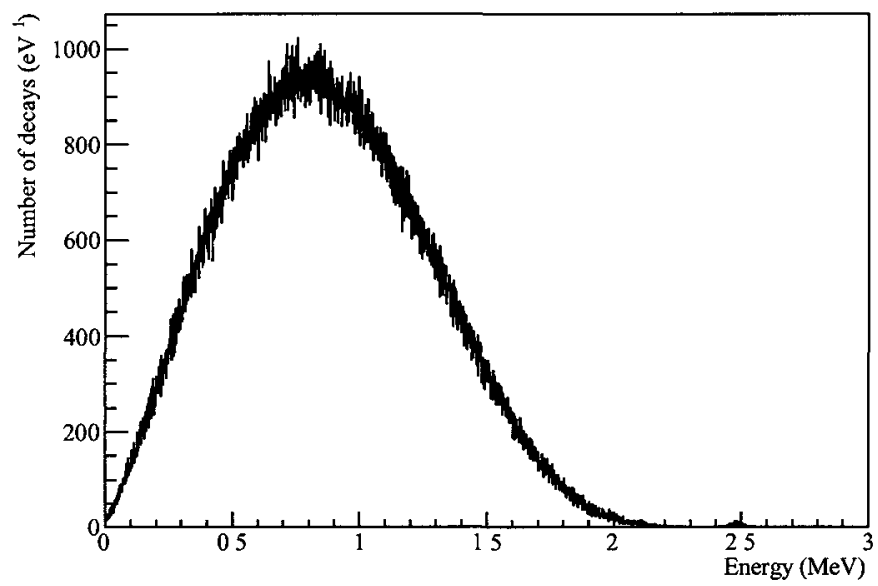

(b)

Figure 2.6: Simulated energy spectra of the $0 \nu \beta \beta$ and $2 \nu \beta \beta$ decays for a one year exposure of one ton of ${ }^{136} \mathrm{Xe}$. Rates are based on the average theoretical half-life values, assuming a $\left\langle m_{\beta \beta}\right\rangle$ of $1 \mathrm{eV}$. The continuum is the $2 \nu \beta \beta$ decay spectrum and the small peak on the right at $2.479 \mathrm{MeV}$ is the $0 \nu \beta \beta$ decay peak. An energy spectrum from a detector with a perfect energy resolution is shown on (a) while a $1 \%$ energy resolution is shown on (b). Notice the difficulty to see the $0 \nu \beta \beta$ peak on (b). An enlarged version of (b) is shown in Figure 2.7a. 


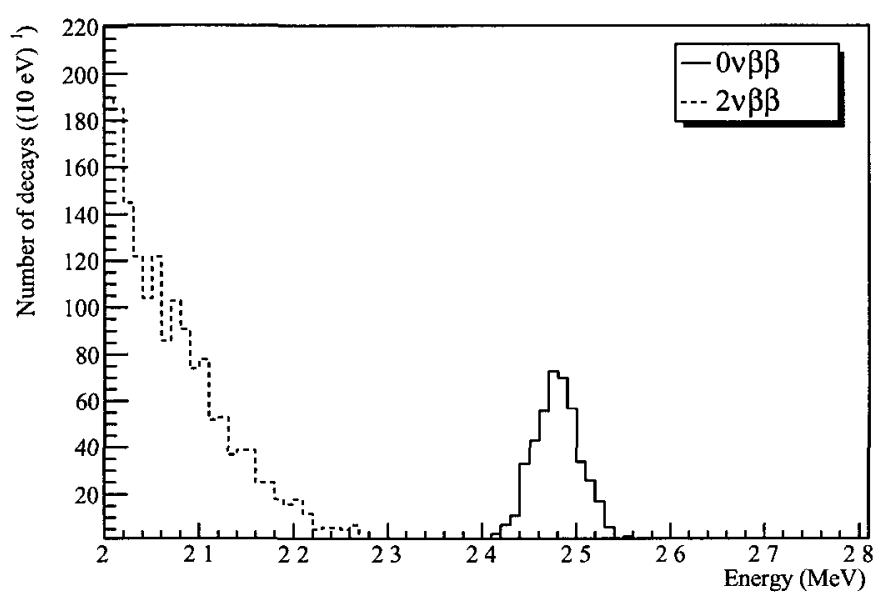

(a)

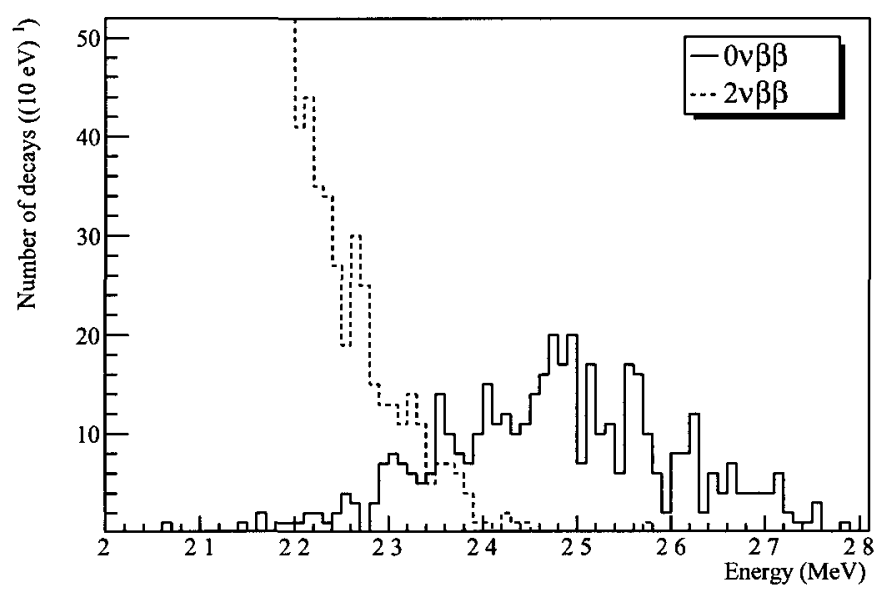

(b)

Figure 2.7: Simulated energy spectra of the $0 \nu \beta \beta$ and $2 \nu \beta \beta$ decays for a one year exposure of one ton of ${ }^{136} \mathrm{Xe}$ enlarged near the $0 \nu \beta \beta$ energy. Rates are based on the average theoretical half-life values, assuming a $\left\langle m_{\beta \beta}\right\rangle$ of $1 \mathrm{eV}$. The solid line distribution is the $0 \nu \beta \beta$ peak and the broken one on the left is the $2 \nu \beta \beta$ distribution. An energy spectrum from a detector with a $1 \%$ energy resolution is shown on (a) while a $5 \%$ energy resolution is shown on (b). 


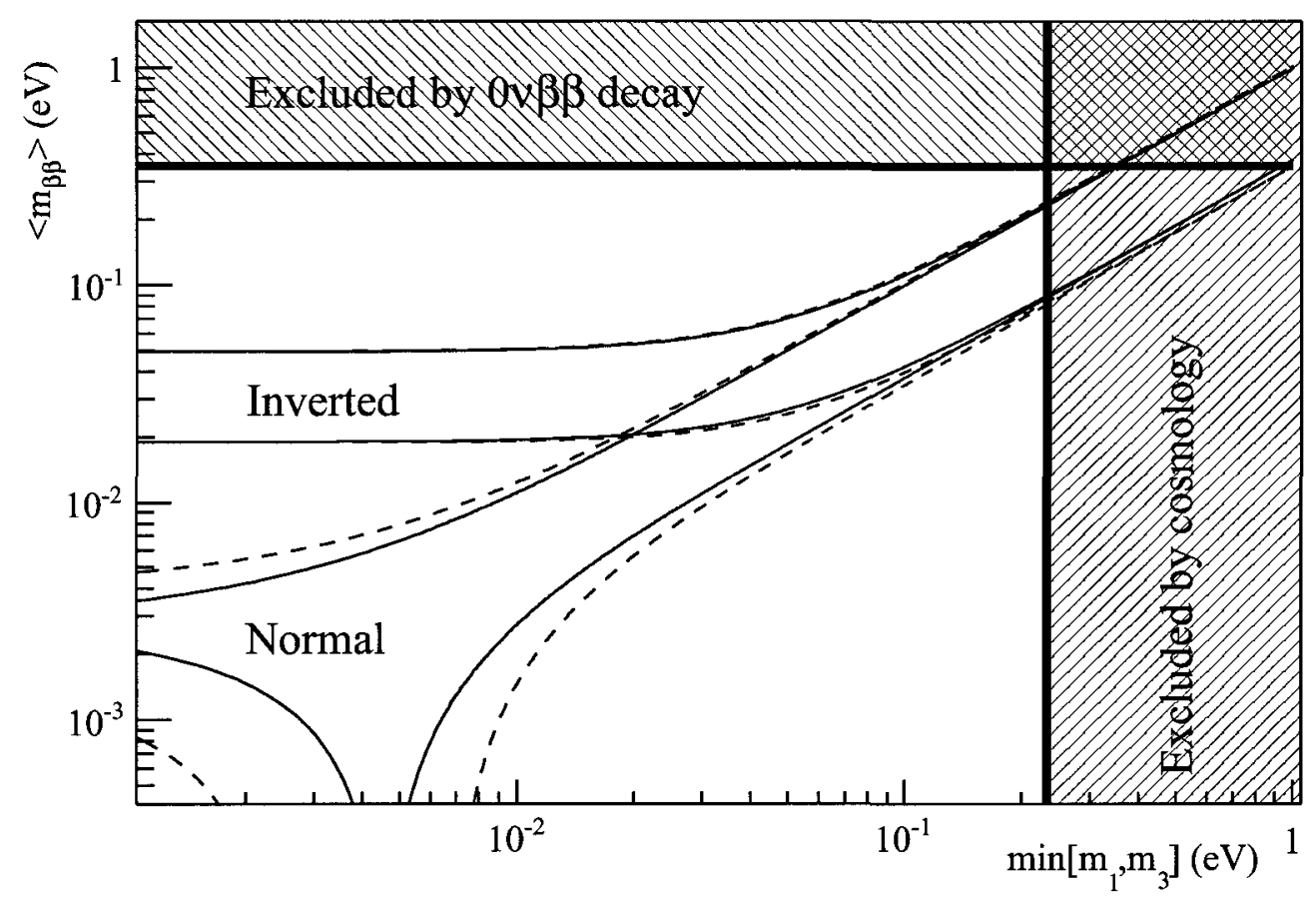

Figure 2.8: Allowed region from neutrino oscillation and cosmology experiments of the $\left\langle m_{\beta \beta}\right\rangle$ and the $\min \left[m_{1}, m_{3}\right]$ parameters. The solid lines correspond to the allowed region for $0 \nu \beta \beta$ assuming a $\sin ^{2}\left(2 \theta_{13}\right)=0$, while the broken lines are contours defined by using the current upper limit of $\sin ^{2}\left(2 \theta_{13}\right)<0.19$. 


\begin{tabular}{|c|c|c|c|}
\hline Isotope & Reference & $\begin{array}{c}\text { Lower limit } \\
\text { on } T_{1 / 2}^{0 \nu \beta \beta}(\mathrm{yr})\end{array}$ & $\begin{array}{c}\text { Upper limit } \\
\text { on }\left\langle m_{\beta \beta}\right\rangle(\mathrm{eV})\end{array}$ \\
\hline \hline${ }^{48} \mathrm{Ca}$ & K. You et al. [27] & $9.5 \times 10^{21}(76 \%)$ & 8.3 \\
\hline${ }^{76} \mathrm{Ge}$ & $\begin{array}{c}\text { H. V. Klapdor-Kleingrothaus } \\
\text { et al. [28] }\end{array}$ & $1.9 \times 10^{25}$ & 0.35 \\
\hline${ }^{76} \mathrm{Ge}$ & C. E. Aalseth et al. [29] & $1.6 \times 10^{25}$ & $0.33-1.35$ \\
\hline${ }^{82} \mathrm{Se}$ & S. R. Elliott et al. [30] & $2.7 \times 10^{22}(68 \%)$ & 5 \\
\hline${ }^{100} \mathrm{Mo}$ & H. Ejiri et al. [31] & $5.5 \times 10^{22}$ & 2.1 \\
\hline${ }^{116} \mathrm{Cd}$ & F. A. Davevich et al. [32] & $7 \times 10^{22}$ & 2.6 \\
\hline${ }^{128} \mathrm{Te}$ & T. Bernatowicz et al. [33] & $7.7 \times 10^{24}$ & $1.1-1.5$ \\
\hline${ }^{130} \mathrm{Te}$ & A. Alessandrello et al. [34] & $1.4 \times 10^{23}$ & $1.1-2.6$ \\
\hline${ }^{136} \mathrm{Xe}$ & R. Luescher et al. [35] & $4.4 \times 10^{23}$ & $1.8-5.2$ \\
\hline${ }^{150} \mathrm{Nd}$ & A. De Silva et al. [36] & $1.2 \times 10^{21}$ & 3 \\
\hline
\end{tabular}

Table 2.6: Current experimental limits on $T_{1 / 2}^{0 \nu \beta \beta}$ as summarized by Elliott and Vogel [37]. Values were calculated with a $90 \%$ confidence level unless explicitly written. A range in $\left\langle m_{\beta \beta}\right\rangle$ means that the authors have been using different matrix elements.

\subsubsection{Other physics}

The light Majorana neutrino propagator might not be the only channel for a $0 \nu \beta \beta$ decay. Other models (left-right symmetric models, extra-dimensions, supersymmetry, etc...) could allow it. If a light neutrino propagator is not the only decay channel of $0 \nu \beta \beta$ decay, Equation 2.5 is no longer useful to measure $\left\langle m_{\beta \beta}\right\rangle$. Nevertheless, the Schechter-Valle theorem [38] shows that no matter what the main $0 \nu \beta \beta$ decay channel is, a positive $0 \nu \beta \beta$ signal automatically implies a Majorana type neutrino. The generic diagram of a $0 \nu \beta \beta$ decay depicted in Figure 2.9 can be rearranged into Figure 2.10. This shows that a $\bar{\nu}$ can go to $\nu$ by such a reaction. Therefore, the knowledge of the mechanism producing $0 \nu \beta \beta$ decay is not necessary to claim the Majorana nature of the neutrino.

The underlying physics of the $0 \nu \beta \beta$ reaction will likely be unveiled by a multidimensional analysis of many experiments, including those described in previous sections and collider experiments, by measuring coupling constants and masses of 
beyond SM particles. Meanwhile, some basic information can be obtained from the $0 \nu \beta \beta$ decay topology. It is possible to differentiate $0 \nu \beta \beta$ decay models from the angular correlation and the energy distribution of the two electrons of the $0 \nu \beta \beta$ decay.

\section{Angular Correlation Analysis}

The angular correlation study has been performed in this thesis framework to justify the use of a tracking detector, like a gas phase EXO experiment, for a $0 \nu \beta \beta$ decay measurement and its possibility to observe physics beyond the SM. A gas phase detector such as described in Section 3.2 would record the electron tracks and allow the electron angular correlation measurement. The analysis uses the theoretical developments of Ali et al. [39], by applying it to a tracking detector for a specific $0 \nu \beta \beta$ decay model.

Discriminating based on the topology of the $0 \nu \beta \beta$ decay will reduce the number of background events by a large amount. A tracking detector could be able to differentiate between two $1 \mathrm{MeV}$ electrons and one $2 \mathrm{MeV}$ electron by their track shape and the energy loss along it. It could also determine if two electrons have the same origin. But there is another incentive to measure the track topology and energy of $0 \nu \beta \beta$ decays.

As stated previously, the light Majorana neutrino propagator might not be the main decay channel for $0 \nu \beta \beta$ decay. Therefore, it is difficult to justify the neutrino mass calculation from a $0 \nu \beta \beta$ decay rate without knowing the main decay channel. The decay rate is a function of the angle between the two electrons and can be written as:

$$
\frac{d \Gamma}{d \cos \theta}=\left|M_{G T}\right|^{2} \frac{\ln 2}{2}(A+B)(1-K \cos \theta) .
$$

$M_{G T}$ is the Gamow-Teller matrix element, $A$ and $B$ are parameters that depend on the $0 \nu \beta \beta$ decay model considered and $\theta$ is the angle between the two electron 


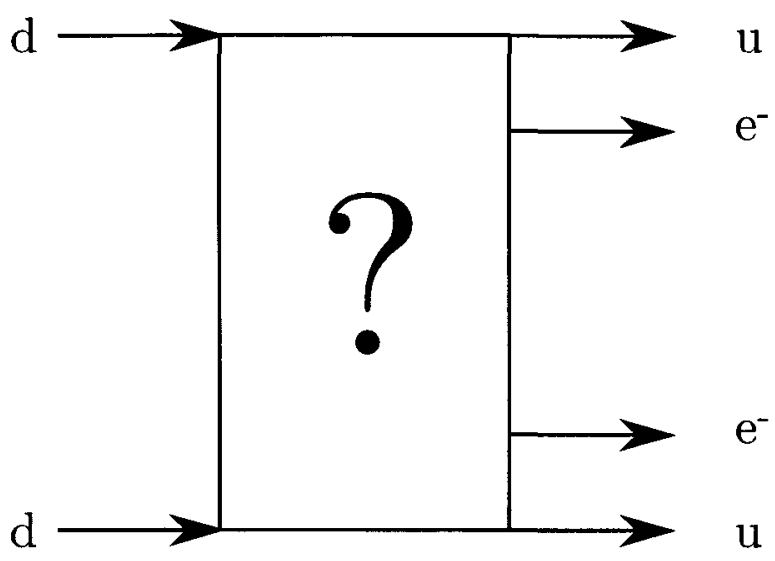

Figure 2.9: Generic diagram for $0 \nu \beta \beta$.

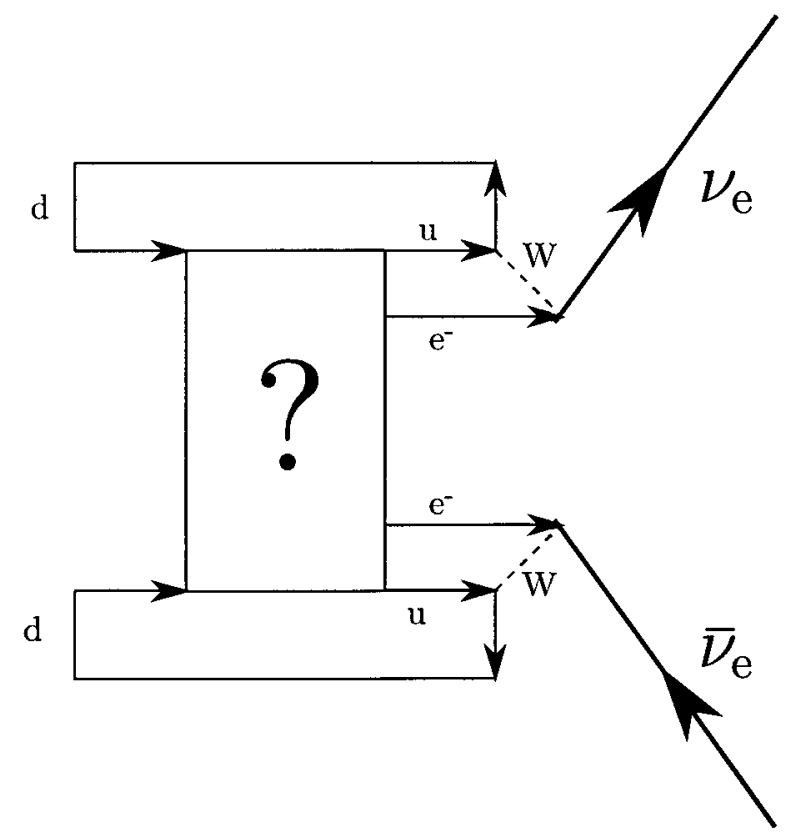

Figure 2.10: Figure 2.9 rearranged to show the Majorana nature of the neutrino assuming $0 \nu \beta \beta$ exists, no matter the type of physics involved in the box. 


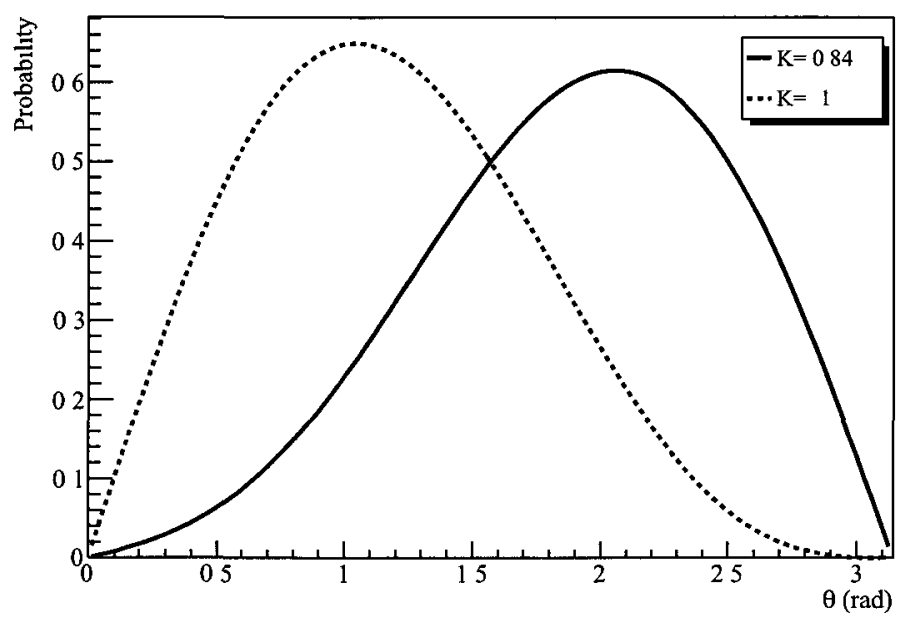

Figure 2.11: Probability density function of the electron angular correlation distribution for $0 \nu \beta \beta$ decays for a light neutrino propagator, with $K=0.84$, and a very different hypothetical model where $K=-1$.

tracks. The parameter $K$ is called the angular coefficient and different $0 \nu \beta \beta$ models will give different values for $K$, with $-1<K<1$. Ali et al. [39] have calculated $K$ for the regular $0 \nu \beta \beta$ decay with a light Majorana neutrino as the propagator and they obtained $K=0.84$ for ${ }^{136} \mathrm{Xe}$. Figure 2.11 shows the angular distribution for two very different values of $K$.

\section{Left-Right Symmetric model}

A model that is likely to give an angular correlation coefficient different from the one from a light Majorana neutrino is the left-right symmetric model. This is due to one of the two weak interactions involved in the decay being right-handed. Both electrons produced would have opposite handedness and their trajectories would be preferably in the same direction instead of back to back like for the light Majorana neutrino propagator. For the left-right symmetric model, the corresponding $K$ is calculated using: 


$$
K=\frac{y-1}{y+1}
$$

where

$$
y=\frac{G_{01}}{G_{02}}\left(\frac{1-\chi_{F}}{\chi_{2-} \epsilon} \frac{|\langle m\rangle|}{m_{e}}\right)^{2}\left(\frac{m_{W_{R}}}{m_{W_{L}}}\right)^{4} .
$$

In Equation 2.8, $G_{0 i}$ are phase space factors, $\chi_{F}$ and $\chi_{2-}$ are parameters of the nuclear matrix element calculations and $\epsilon$ is related to the Dirac mass scale divided by the massive $\nu_{R}$ scale. $m_{e}, m_{W_{R}}$ and $m_{W_{L}}$ are the mass of the electron and the right and left handed $W$ bosons. Current measured or estimated values of those parameters are listed in Table 2.7.

\section{Sensitivity to $m_{W_{R}}$ with a tracking experiment}

One can ask how sensitive a tracking detector will be to the mass of a $W_{R}$ in a left-right symmetric model. The current lower limit on the $W_{R}$ mass of $715 \mathrm{GeV}$ at $90 \%$ C.L. comes from the LEP electroweak fit [40]. A good angular resolution is important but not critical for a measurement of $K$ because of the smooth appearance of the angular distribution as shown by Figure 2.11. This analysis has the goal to calculate the amount of data needed to reach the limit of $715 \mathrm{GeV}$ for $M_{W_{R}}$, as a function of $\left\langle m_{\beta \beta}\right\rangle$. The procedure was very simple. The mean of the correlation angle distribution $(\bar{\theta})$ was found to be a good estimator of the parameter $K$ using the Monte Carlo method. For a deterctor with a perfect resolution, $K$ can be calculated from $\bar{\theta}$ with:

$$
K=(\bar{\theta}-1.57) / 0.39
$$

Then, 10,000 experiments were generated for a given angular correlation, again using the Monte Carlo method. For each experiment, $K$ was reconstructed and the standard deviation of the $K$ distribution $\left(\sigma_{K}\right)$ was calculated. The 10,000 


\begin{tabular}{|c|c|}
\hline Parameter & Value \\
\hline \hline$G_{01}$ & $7.928 \times 10^{-15} \mathrm{yr}^{-1}$ \\
\hline$G_{02}$ & $12.96 \times 10^{-15} \mathrm{yr}^{-1}$ \\
\hline$\chi_{F}$ & 0.274 \\
\hline$\chi_{2-}$ & 0.551 \\
\hline$\epsilon$ & $10^{-6}$ \\
\hline$m_{e}$ & $511 \mathrm{keV}$ \\
\hline$m_{W_{L}}$ & $80.4 \mathrm{GeV}$ \\
\hline
\end{tabular}

Table 2.7: Value of the parameters used in the angular correlation analysis.

experiments were repeated with a different number of events or a different angular resolution and every $\sigma_{K}$ was recorded. Then, an analytical equation has been fitted allowing the calculation of $\sigma_{K}$ from the angular resolution and the number of events. The equation obtained is:

$$
\log _{10} \sigma_{K}=-\log _{10}(\sqrt{n})+0.13\left(\sigma_{\theta}\right)^{2}-0.61\left(\sigma_{\theta}\right)+0.81
$$

where $n$ is the number of $0 \nu \beta \beta$ events and $\sigma_{\theta}$ is the angular resolution of the detector in radians. Finally, the number of years required to reach the $M_{W_{R}}$ limit has been calculated using values in Table 2.7. It assumes a decay rate of 1.1 ton $^{-1} \mathrm{yr}^{-1}$ at $50 \mathrm{meV}$, but was scaled for different values of $\left\langle m_{\beta \beta}\right\rangle$.

The result is shown in Figure 2.12 for four different angular resolution scenarios. It is clear that it will be very challenging to perform a measurement of a $W_{R}$ mass. Two processes compete to make this measurement difficult. At low $\left\langle m_{\beta \beta}\right\rangle$ the event rate is too low to reach the statistical significance in the measurement and at high $\left\langle m_{\beta \beta}\right\rangle$ the decay looks too much like a light neutrino propagator $0 \nu \beta \beta$ decay. Nevertheless, other theories can be tuned to give a very different $K$ value, even at high $\left\langle m_{\beta \beta}\right\rangle$, so measuring the angular correlation coefficient is a real advantage of tracking detectors, for the discovery of new physics. 


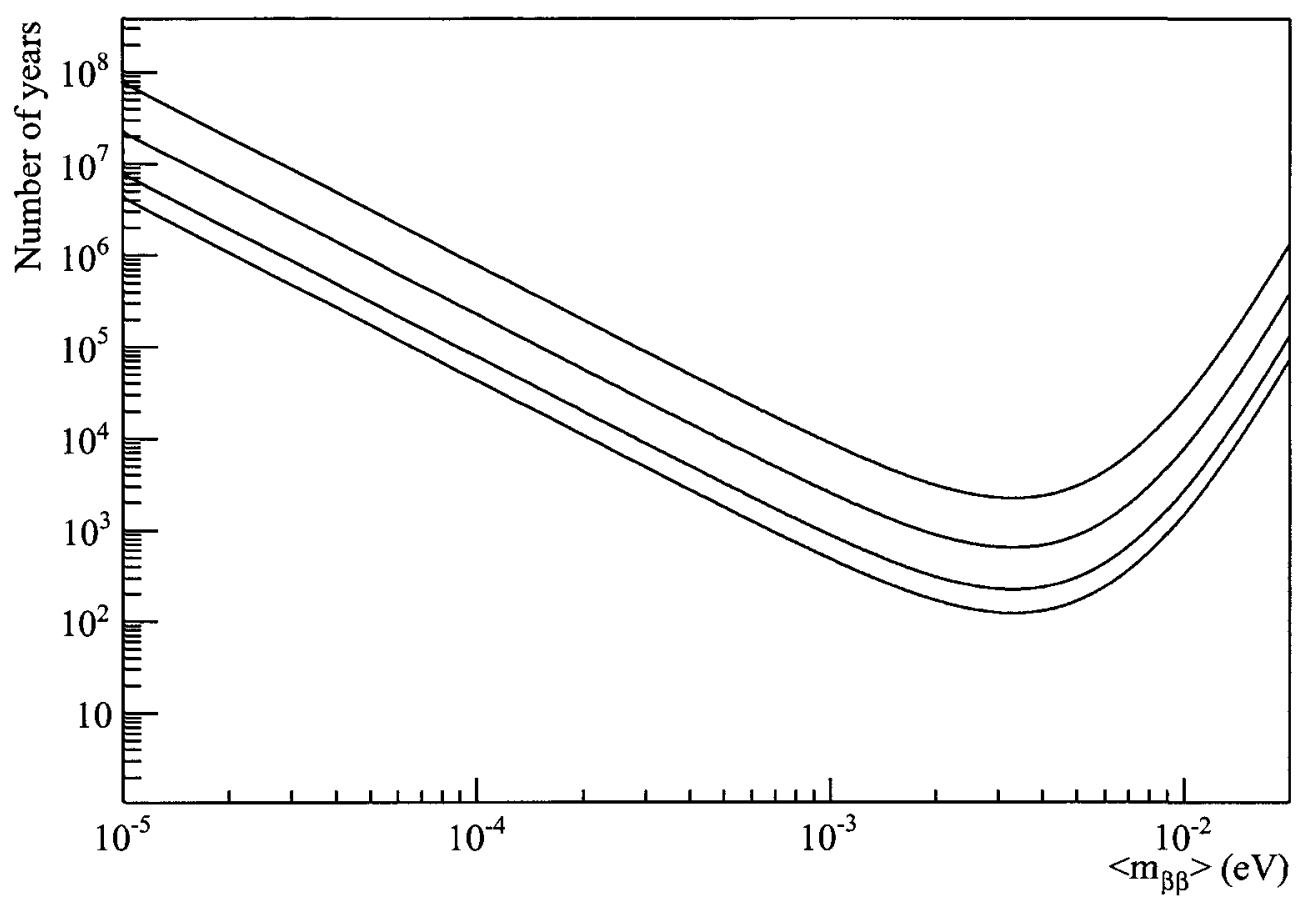

Figure 2.12: Projection of the expected number of years required to reach the LEP limit on the $W_{R}$ mass. Calculations have been done with 1 ton of enriched xenon. The only uncertainty is the angular resolution of the detector. The top curve shows a $90^{\circ}$ uncertainty, the next one $60^{\circ}$, then $30^{\circ}$ and finally the bottom curve shows the number of years required to reach the LEP limit on the mass of the $W_{R}$ for a perfect detector. 


\section{Chapter 3}

\section{Enriched Xenon Observatory}

One of the major experiments designed to measure the $0 \nu \beta \beta$ rate is the Enriched Xenon Observatory (EXO) experiment. The concept is a time projection chamber (TPC) filled with ${ }^{136} \mathrm{Xe}$. The two electrons created by $0 \nu \beta \beta$ will ionize xenon and the number of secondary electrons will indicate the total decay energy. Xenon has several advantages over other isotopes for a $0 \nu \beta \beta$ measurement. It can be easily filtered to remove impurities. Both chemical traps and cryogenic traps can be used since xenon is an inert gas and has a condensation temperature of $165 \mathrm{~K}$ significantly different than common contaminants. Xenon can also be enriched to increase the amount of active isotope. It can be used as the $0 \nu \beta \beta$ source and as the active medium of the TPC to perform the energy measurement. The decay also releases a large amount of energy, making the energy measurement more accurate and much larger than the more numerous lower energy background events. And finally, a large reduction of the background is possible if barium tagging is performed.

EXO is not the first group attempting to measure the $0 \nu \beta \beta$ decay rate. In the 90 's, the Gotthard collaboration built a gas TPC for this purpose [35]. They used $3.3 \mathrm{~kg}$ of Xe enriched at $62.5 \%$, filling a $180 \mathrm{~L}$ vessel at $5 \mathrm{~atm}$. The ionization signal was recorded on an $\mathrm{x}-\mathrm{y}$ grid and allowed for the reconstruction of the ionization 
tracks. The experiment collected a total of 12843 hours of data and placed a limit on $T_{1 / 2}^{0 \nu \beta \beta}>4.4 \times 10^{23} \mathrm{yr}$. For the $2 \nu \beta \beta$ decay, they analyzed the 1750 to $2000 \mathrm{keV}$ energy window and were able to place a limit of $T_{1 / 2}^{2 \nu \beta \beta}>3.6 \times 10^{20} \mathrm{yr}$, within one order of magnitude of the theoretical prediction. The energy resolution measured by the full width at half maximum (FWHM) was $8 \%$ at $1592 \mathrm{keV}$, or $6.6 \%$ at the $0 \nu \beta \beta$ energy if scaled linearly.

For the EXO experiment, two options are currently studied, one using xenon in its liquid phase and the second in its gas phase. The advantage of using liquid is the size of the experiment. For example, $200 \mathrm{~kg}$ of liquid xenon occupies $0.07 \mathrm{~m}^{3}$ while in its gas phase, the same mass fills a volume of $38 \mathrm{~m}^{3}$ at atmospheric pressure and room temperature. On the other hand, a gas phase TPC would present other advantages. First, the energy resolution of the ionization yield is believed to be better than in the liquid phase, an important parameter in the reduction of the $2 \nu \beta \beta$ events in the $0 \nu \beta \beta$ energy window. Second, the two electron track topology can be recorded, which yields a clear advantage in background event reduction and allows the physics analysis of the nature of the virtual particles exchanged in $0 \nu \beta \beta$. Finally, there is no need to cool the detector which reduces the amount of material in the vicinity of the fiducial volume which decreases the background event rate.

An initial gas prototype has been described in an early EXO publication [41]. The design proposed was a $5 \mathrm{~m}$ long cylinder with a diameter of $3.2 \mathrm{~m}$. At $5 \mathrm{~atm}$, the volume would have contained 1 ton of xenon. The ionization signal was supposed to be amplified with Gas Electron Multiplier (GEM) plates and the xenon scintillation was collected by photomultiplier tubes (PMT) at the periphery of the xenon volume. Barium tagging was planned to be performed in situ, by aiming a laser beam with galvanometer driven mirrors or acousto-optic beam deflectors and the fluorescence signal would have been collected by the PMTs. The exact tagging procedure was not selected, although some techniques were outlined. Chapter 4 of this thesis reviews these techniques and, after some research, shows that most of 
them are not practical in high pressure gas. The current design of a gas prototype, described in Section 3.2, is very different than the original one.

\subsection{Liquid Xenon Prototype}

To determine which option is the most capable, two prototypes are being developed. The most mature one is the liquid phase detector, called EXO-200. It is currently in its commissioning phase and should start taking data this year using $200 \mathrm{~kg}$ of enriched xenon. The main goals of the prototype are, beside testing the technology, to measure the $2 \nu \beta \beta$ decay rate and to test the measurement of the Klapdor-Kleingrothaus group of the $0 \nu \beta \beta$ decay rate.

The detector was built underground at the Waste Isolation Pilot Plant (WIPP) in New Mexico. A model of the experiment is shown in Figure 3.1 and Figure 3.2 shows details of the actual TPC. The detector records the ionization by drifting the secondary electrons toward conductive grids, and the charge induced and collected by them indicates the energy of the ionizing particles. The detector also records the scintillation light, generated by the excitation of the xenon atoms by the primary electrons, using avalanche photodiodes (APD). The EXO collaboration has measured an anti-correlation between the charge and the light measurements [42]. If this effect is taken into account during the analysis, it increases the energy resolution of the detector. Barium tagging is not included in the EXO-200 prototype. Nevertheless, research and development is ongoing to build a probe that grabs the barium ion, extracts it from the xenon volume and releases it in a trap. Another option investigated would perform the tagging in the liquid xenon with an optical fibre. It would capture the ion by freezing it, carry the excitation laser light and returns fluorescence signal from the ion. 


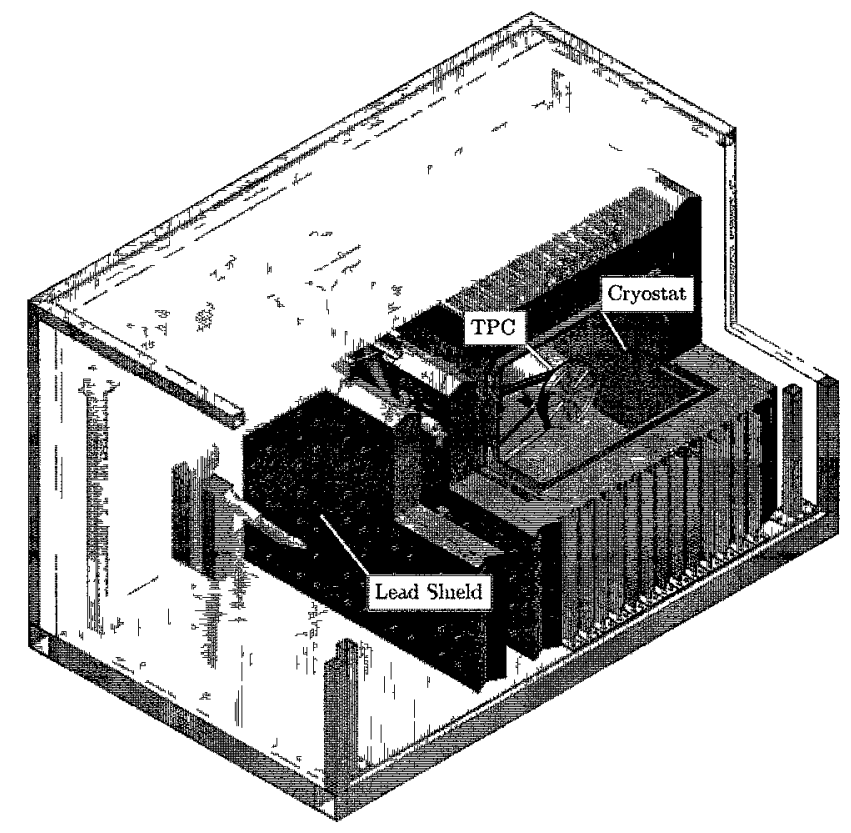

Figure 3.1: Schematics of the EXO-200 experiment. The cylindrical TPC is in the centre of a large cryostat, itself embedded in a lead structure to reduce the background event rate.
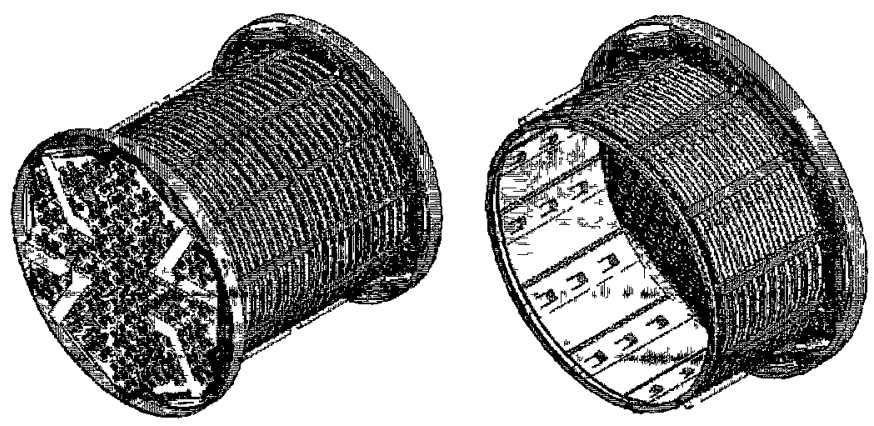

Figure 3.2: Schematics of the EXO-200 experiment TPC. The full cylindrical TPC is on the left and a cut version is displayed on the right. Field shaping rings are displayed along the cylinder length. Some APDs are visible as circles inside the cut view. 


\subsection{Gas Xenon Prototype}

The second prototype uses xenon in its gas phase. Part of the EXO collaboration is currently building the Xenon Electroluminescent Prototype (XEP) at Carleton University. It is a $1 \mathrm{~m}^{3}$ gas TPC operated at pressures between 1 and 10 bar. Figure 3.3 illustrates the TPC. The plan is to use $20 \mathrm{~kg}$ of non-enriched pure xenon. It is possible that argon or neon could be mixed with xenon to determine the conditions for the best energy resolution and smoother electron tracks. The current concept requires to measure the electroluminescence (EL) light from secondary electrons, created when accelerated by a very large electric field (> $6000 \mathrm{~V} / \mathrm{cm}$ at $1 \mathrm{~atm})$. A relocation to an underground laboratory and an upgrade to enriched xenon are also envisioned.

After the ionizing particle goes through the gas, the secondary electrons from the ionization are drifted to the intense electric field region, on the left of Figure 3.3. The drift field is generated by 27 electrode rings and can reach $1000 \mathrm{~V} / \mathrm{cm}$. The EL region is $1 \mathrm{~cm}$ wide and the electric field can be as high as $70,000 \mathrm{~V} / \mathrm{cm}$. Once in the EL region, electrons will create ultraviolet (UV) photons, up to an intensity of 1000 photons/electron. Two caesium iodide photon detectors, one at each end of the drift region, record the light intensity. Each light detector has a specific function. The one next to the EL region will be able to record the spacial distribution of the secondary electrons, enabling a primary track reconstruction. The second light detector, at the other end of the TPC, will record a smooth light distribution, less prone to track topology biases or detector non-uniformities. This will allow to perform an energy measurement of ionizing events with a greater accuracy. To increase the amount of photons reaching the second light detector, a Teflon cylinder will be surrounding the electrode rings. Teflon is known to reflect UV light.

The amount of ${ }^{136} \mathrm{Xe}$ in XEP will not be large enough to measure the $0 \nu \beta \beta$ decay rate. In fact, even a $2 \nu \beta \beta$ decay rate measurement will be a challenge since 


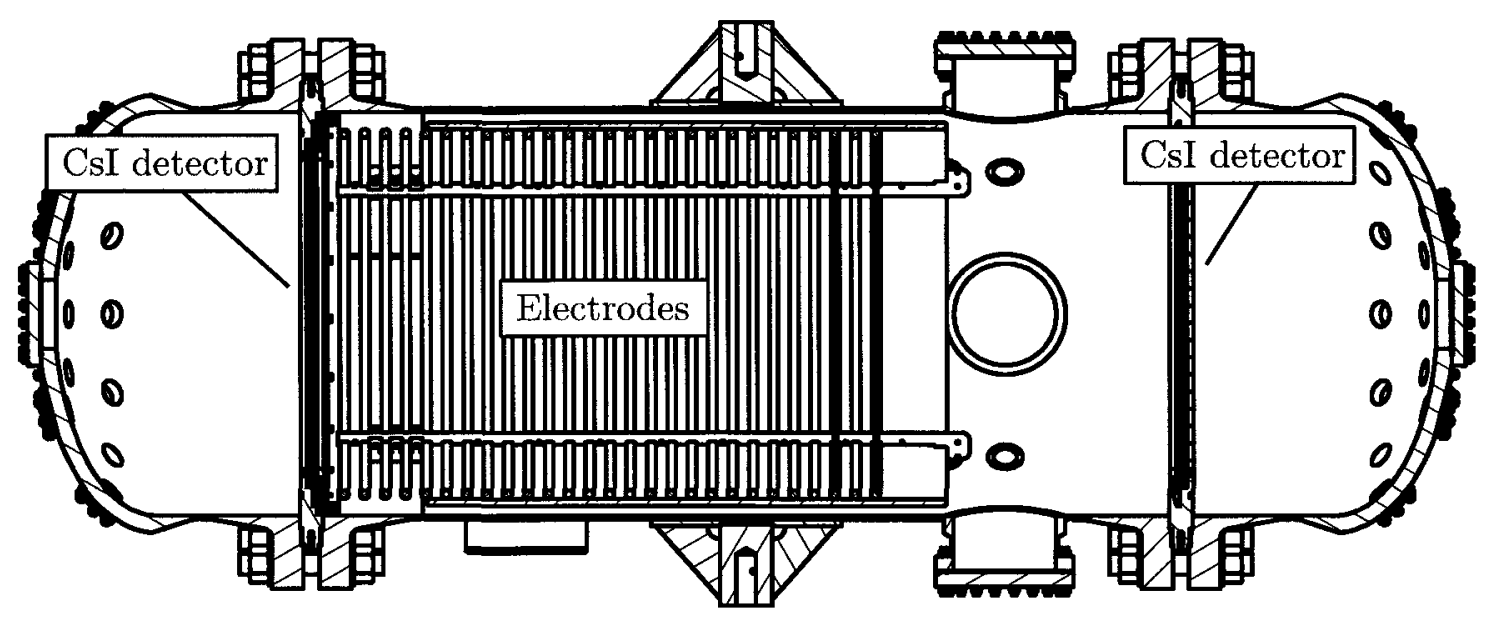

Figure 3.3: Schematics of the XEP chamber. The cylindrical TPC is $210.8 \mathrm{~cm}$ long and has a diameter of $83.1 \mathrm{~cm}$. The central vertical lines are the 27 electrode rings and both CsI photodetectors are located at the extremities of the TPC where the main cylinder is joined with the two bell shaped flanges. The EL region is just next to the CsI photocathode on the left.

only 1000 decays are expected per year of data taking, and only a small fraction of them will occur in the centre of the detector, the location required for a full energy contained event. The background rate should also be high. The detector will be operated on the surface, exposed to muons and other high energy charged particles. In addition, the prototype materials were not selected for low radioactivity content. Nevertheless, the goal of this prototype is to test the concept of EL in xenon and define the operating conditions for a precise energy measurement. It could also be used to test the extraction efficiency of barium ions out of the xenon volume in a trap, through an aperture.

\subsubsection{Track topology}

Important considerations in the design of a gas phase EXO experiment are the topology and the containment of tracks. A track that exits the instrumented 
volume will systematically get a lower reconstructed energy, depending on the fraction of electrons that will drift outside the volume. Since the reconstructed energy is essential to discriminate between $0 \nu \beta \beta$ and $2 \nu \beta \beta$ decays, great care has to be taken in the design of the detector. The track length depends on the gas content, the gas pressure, the energy of the ionizing particle and its type. GEANT4 is the perfect framework to simulate beta events and decide on the optimal shape of the detector. GEANT4 is a program in $\mathrm{C}++$ that allows to create particles and study their interactions in matter through Monte Carlo simulations. The following work has been performed as a first step in the design of the XEP chamber, seen on Figure 3.3.

The simulations were very rudimentary. The detector was created from an infinite volume filled with gas, without any boundaries. Electron tracks were generated at various energies and were allowed to propagate until they stopped. No other processes were simulated, only the primary track and significant secondaries like gamma rays and high energy secondary electrons. Then, the tracks were saved in a file. Different chamber geometries were tested a posteriori for containment. A typical track is shown in Figure 3.4. It was simulated using an electron with an energy of $1 \mathrm{MeV}$ in $10 \mathrm{~atm}$ of xenon. The darkness of the rectangles shows the energy deposited in the gas, mainly through ionization. The electron origin is at $(0,0,0)$.

From the simulated tracks, four tests were performed to determine the TPC dimensions. Those tests are the track propagation distance, the percentage of tracks having $99 \%$ of their energy contained in the detector volume, the number of collisions changing the direction of the track by more than 45 degrees and the number of high energy gammas leaving the detector volume. These were recorded for different gas types, pressures and electron energies. 


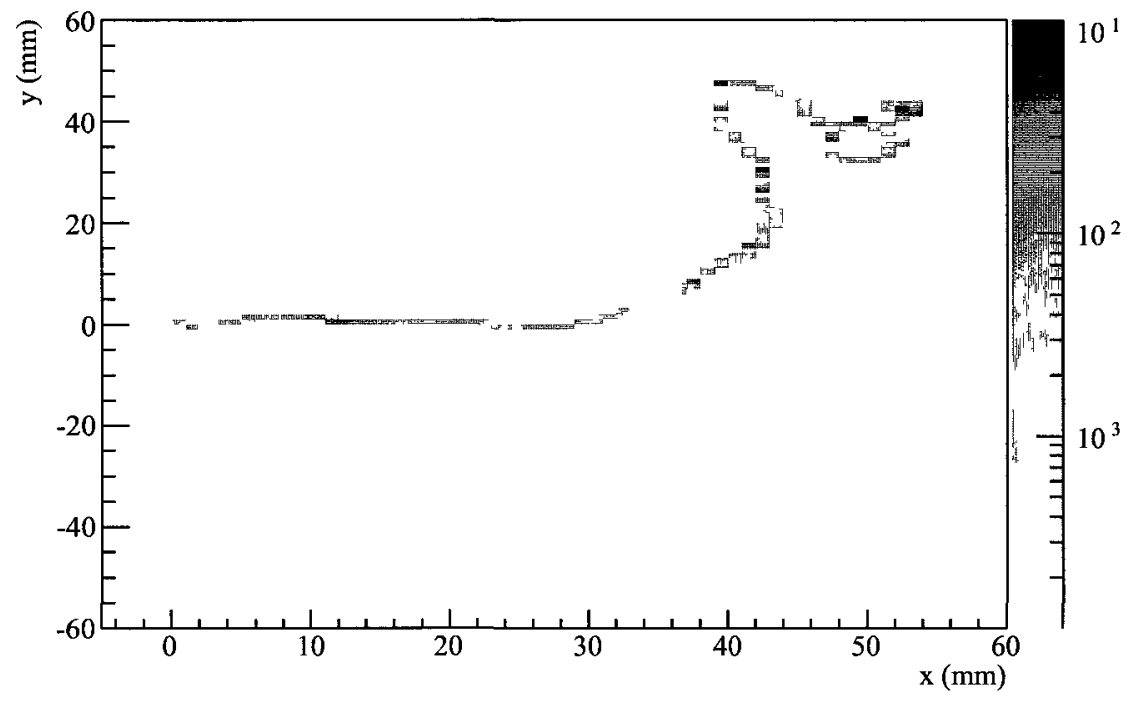

(a)

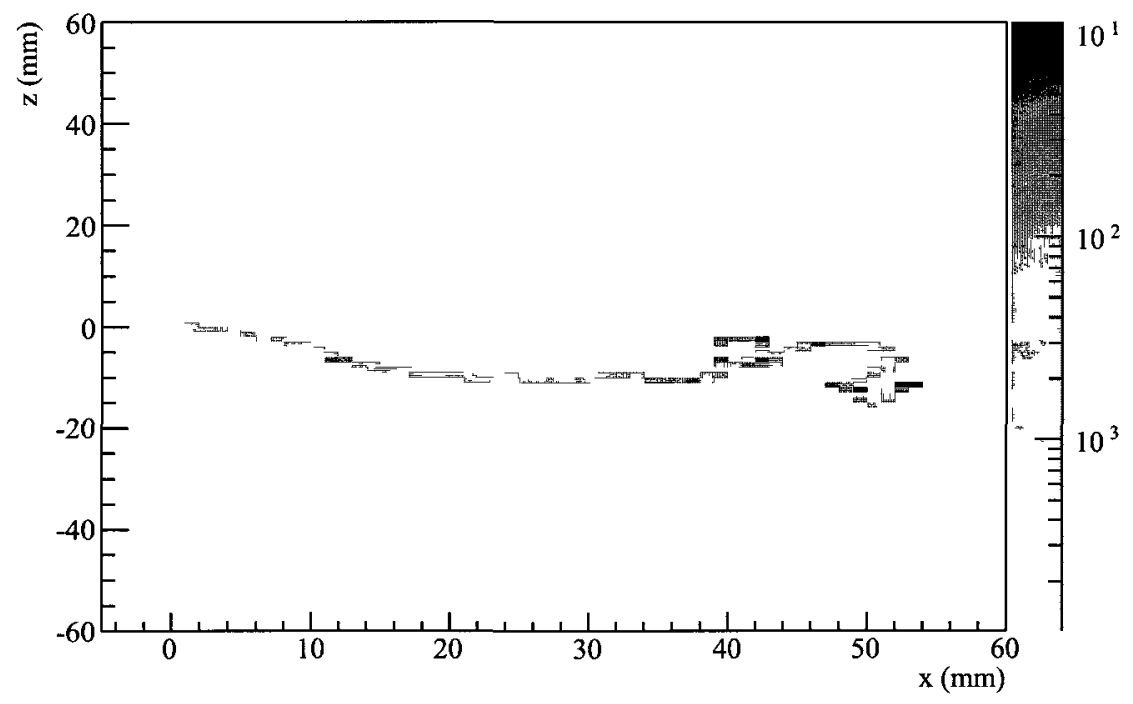

(b)

Figure 3.4: A typical track for a $1 \mathrm{MeV}$ electron in $10 \mathrm{~atm}$ of xenon as projected in the (a) $x-y$ plane and (b) the $x-z$ plane. The darkness of the bin represents the energy loss in $\mathrm{MeV} / \mathrm{mm}^{2}$ for $1 \mathrm{~mm} \times 1 \mathrm{~mm}$ bins. The origin of the electron is at $(0,0,0)$. 


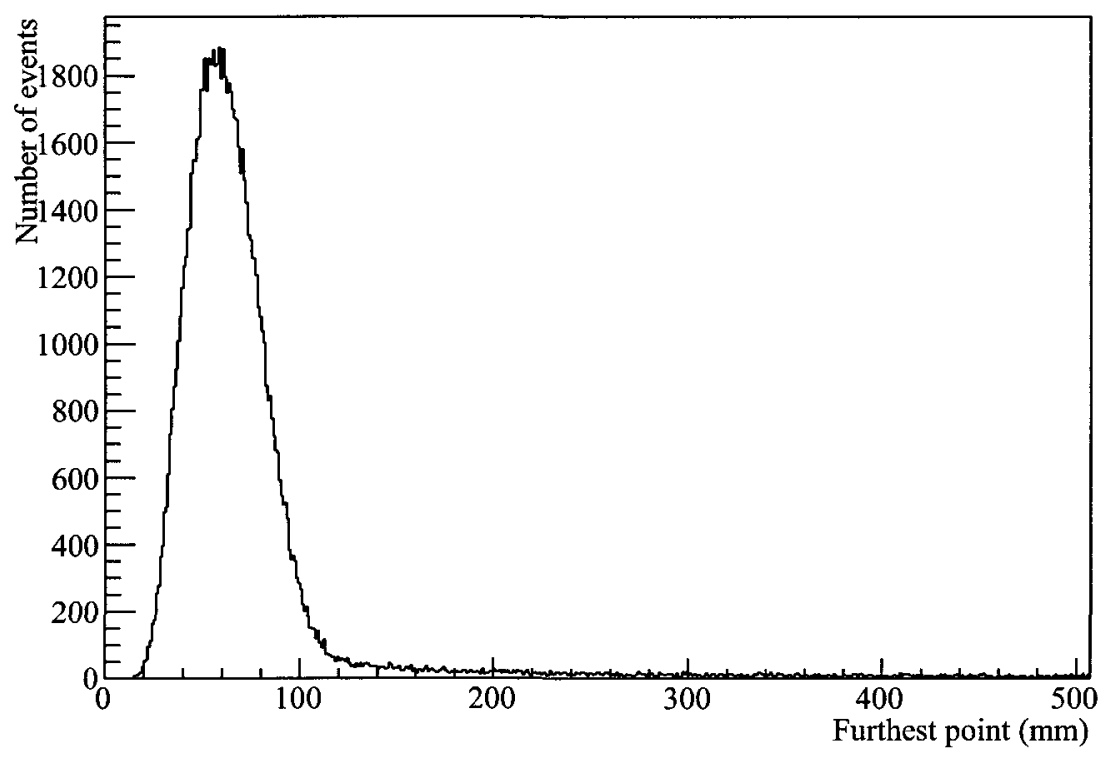

Figure 3.5: Maximum track distance from the origin for $1 \mathrm{MeV}$ electrons in $10 \mathrm{~atm}$ of xenon.

\section{Track propagation distance}

An easy way to measure the efficiency of the containment is to measure the distance of the furthest energy deposition with respect to the source. Most $1 \mathrm{MeV}$ events will be contained by a meter size vessel at a xenon pressure above $1 \mathrm{~atm}$. The only culprits are events where a bremsstrahlung gamma is created and travels for many meters outside the xenon volume. An histogram of the furthest distance of energy deposition for 10,000 electrons with an individual energy of $1 \mathrm{MeV}$ in $10 \mathrm{~atm}$ of xenon is shown as an example in Figure 3.5.

Figure 3.6a shows the mean of the maximum track distance from the origin for xenon, argon and neon at different gas pressures. Figure 3.6b shows the same thing for different electron energies in pure xenon. As expected, the heaviest gas has the best stopping power, because of the larger electron density. Similarly, an 
ionizing electron will take more time to stop if it has a higher energy.

\section{Energy containment}

A more important test is to measure how much energy is contained in the instrumented region. If some of the ionization is lost, the $0 \nu \beta \beta$ decay signal will be burried under the $2 \nu \beta \beta$ continuum and no measurement will be possible. The detector was modelled as a $400 \times 400 \times 600 \mathrm{~mm}$ box, with the electron source at the centre of the $400 \times 400 \mathrm{~mm}$ square and at one end of the $600 \mathrm{~mm}$ side. Electrons were monoenergetic and their direction was fixed to be perpendicular to the $400 \times 400 \mathrm{~mm}$ plane and into the box. Figure 3.7a shows the projection of several tracks of $1 \mathrm{MeV}$ electrons in $10 \mathrm{~atm}$ of xenon. Then, the energy was reconstructed by including only the energy deposited in the box. It is important to note that the simulated data does not consider diffusion, electron amplification, electron attachment or any other experimental effects. An arbitrary $2 \%$ energy Gaussian smearing was introduced as a simple way to incorporate these effects. The resulting distribution is shown in Figure 3.7b.

There are two main causes for the missing energy. The first one is from gamma radiation that leaves the fiducial volume carrying energy with it. The second one is from the primary electron that leaves the source, collides and moves backward, hitting the source holder. Note that the tracks are generally directed forward but a non-negligible number of tracks are scattered and deposit energy behind the source.

From the same simulation, Figure 3.8 shows the fraction of electron events that has $99 \%$ of their energy deposited in the xenon volume for different gas types and electron energies. For Figure 3.8a, three characteristics are displayed. First, the detector will not contain the total amount of energy unless the pressure is large enough. Then, a heavier gas will stop the electrons more efficiently. And finally, a lighter gas can provide a higher containment fraction at large pressures because of the shape of the electrons tracks. As the next section will show, a light gas 


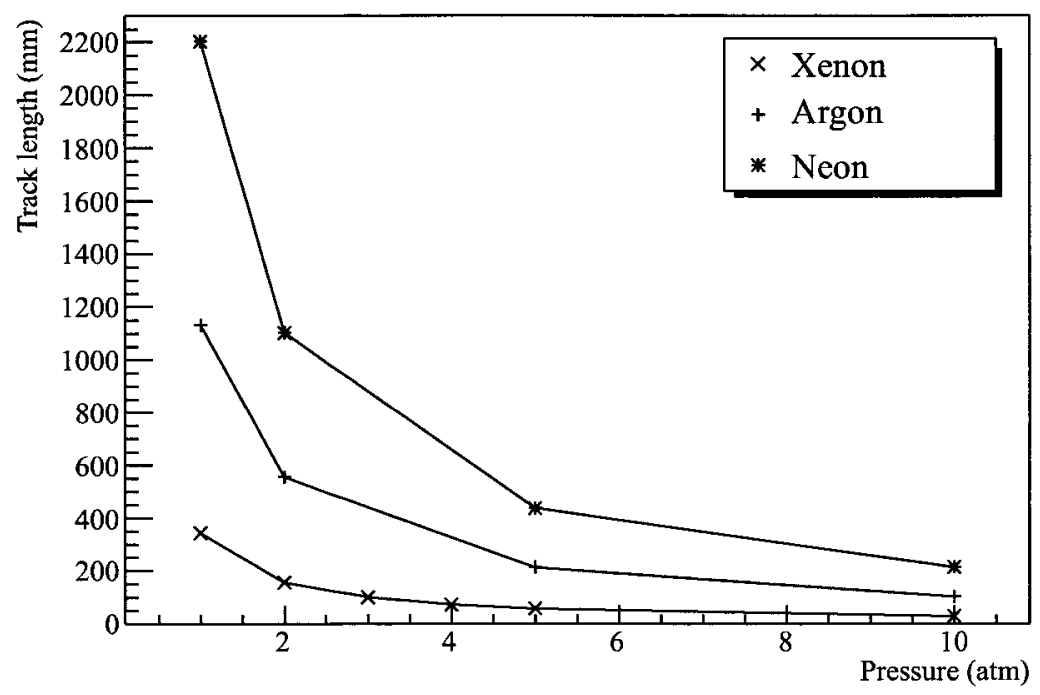

(a)

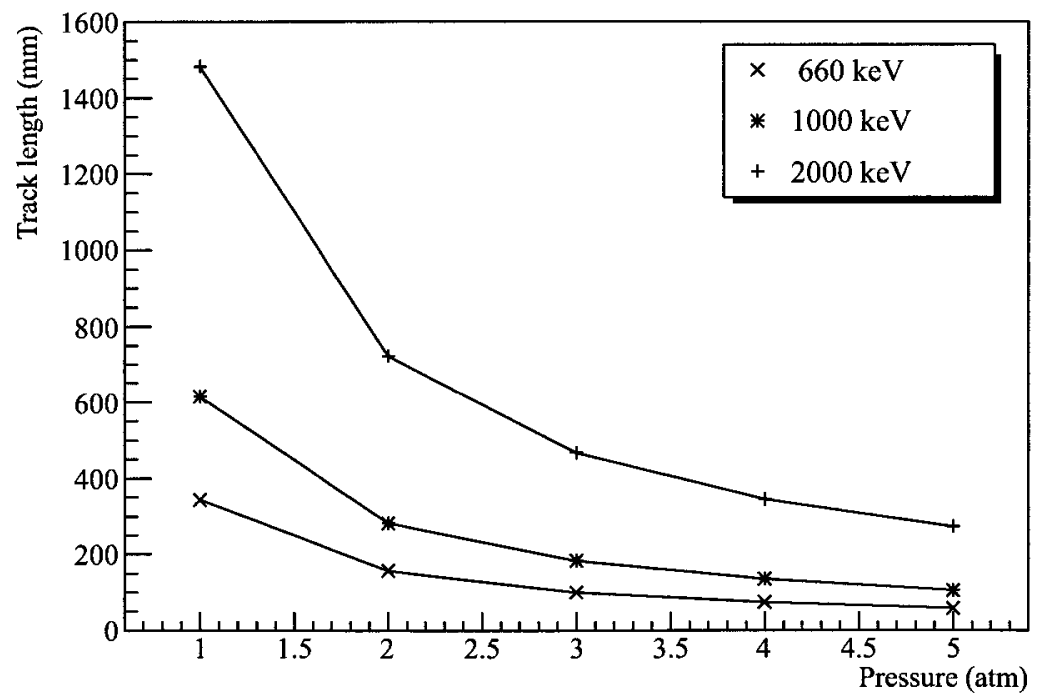

(b)

Figure 3.6: Average maximum distance of tracks for different gas type and electron energies as a function of pressure. Simulation for (a) comes from $660 \mathrm{keV}$ electrons and simulations for (b) comes from pure xenon. 


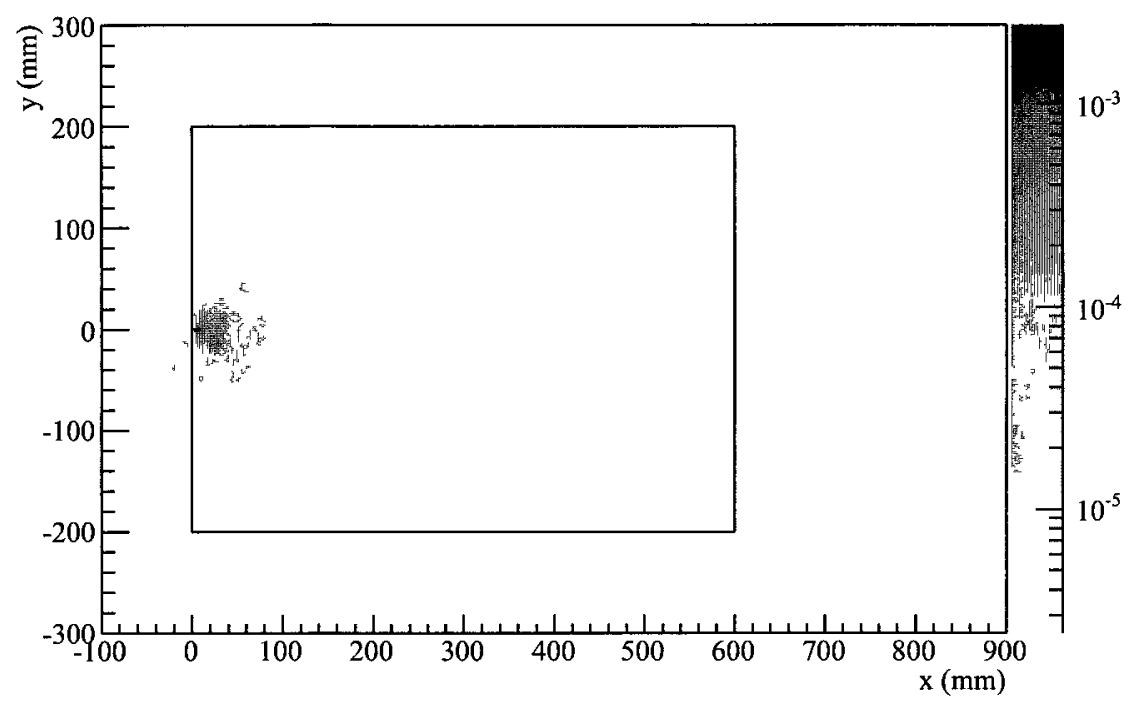

(a)

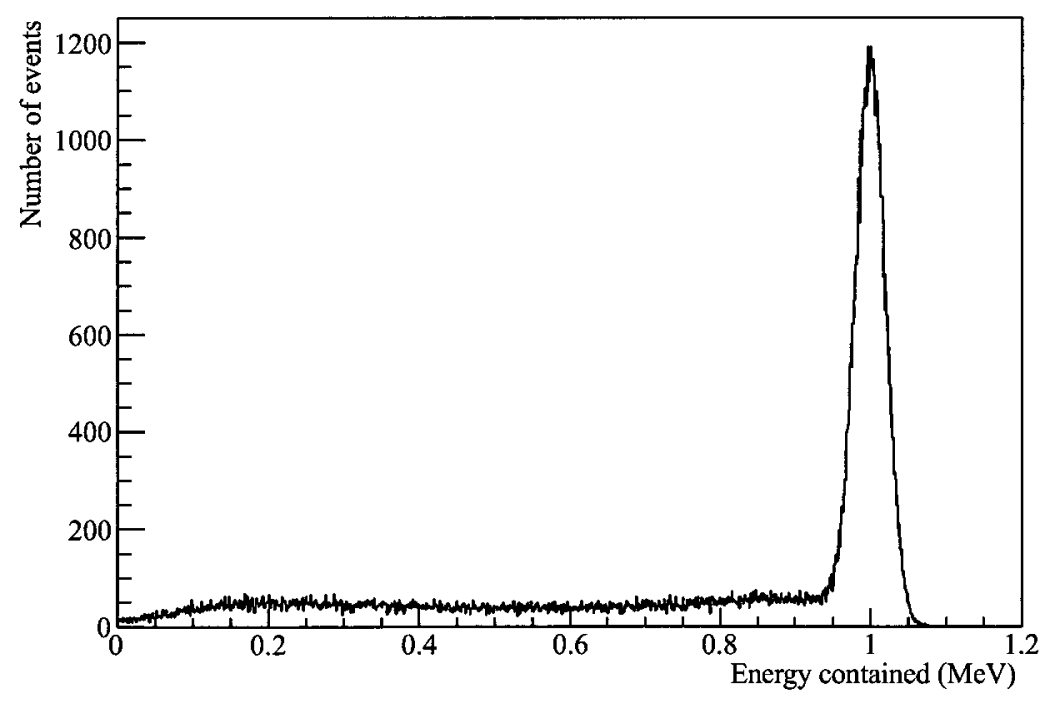

(b)

Figure 3.7: Track density (a) and reconstructed energy (b) for $1 \mathrm{MeV}$ electrons in $10 \mathrm{~atm}$ xenon. 
has fewer hard collisions. In xenon, the primary electron has a greater chance to collide in the first few $\mu \mathrm{m}$ and moves backwards than in neon.

The logical improvement to the design of the TPC would be to move the source inside the volume, with a thin post for example. By shifting the source by $100 \mathrm{~mm}$, the energy containment can be enhanced by a large amount. Figure 3.9 shows the same information as in Figure 3.7, but with the shifted source. The low energy tail of the energy distribution almost disappears and there is a gain of almost $50 \%$ in the number of events contained.

\section{Number of Large Angle Collisions}

In order to determine the vertex of the $0 \nu \beta \beta$ or the $2 \nu \beta \beta$ decay, it is preferable to have a smooth track with a low number of large angle collisions. A large angle collision is defined here as a collision that results in a change in direction larger than 45 degrees, with the energy of the primary electron larger than $10 \mathrm{keV}$. The vertex of the $0 \nu \beta \beta$ decay can be found by looking at the variation in the energy loss along the track. A sharp angle in the track could also be used to locate the origin of the decay as the two primary electrons might not be generated back to back.

The heavier the surrounding gas is, the more large angle collisions the primary electrons will undergo. This effect is shown in Figure 3.10. Xenon is obviously required to observe $0 \nu \beta \beta$ decay, but if mixed with a lighter gas, the conditions for track reconstruction could be improved.

\section{Number of Bremsstrahlung Gammas Produced}

When a high energy photon is generated by the primary electron, mainly from the bremsstrahlung effect, there is a non negligible chance that it will leave the detector volume undetected, carrying with it some of the energy. The resulting reconstructed energy is lower and, in a $0 \nu \beta \beta$ decay experiment, such an event would be outside the energy window analyzed and would be buried under the 


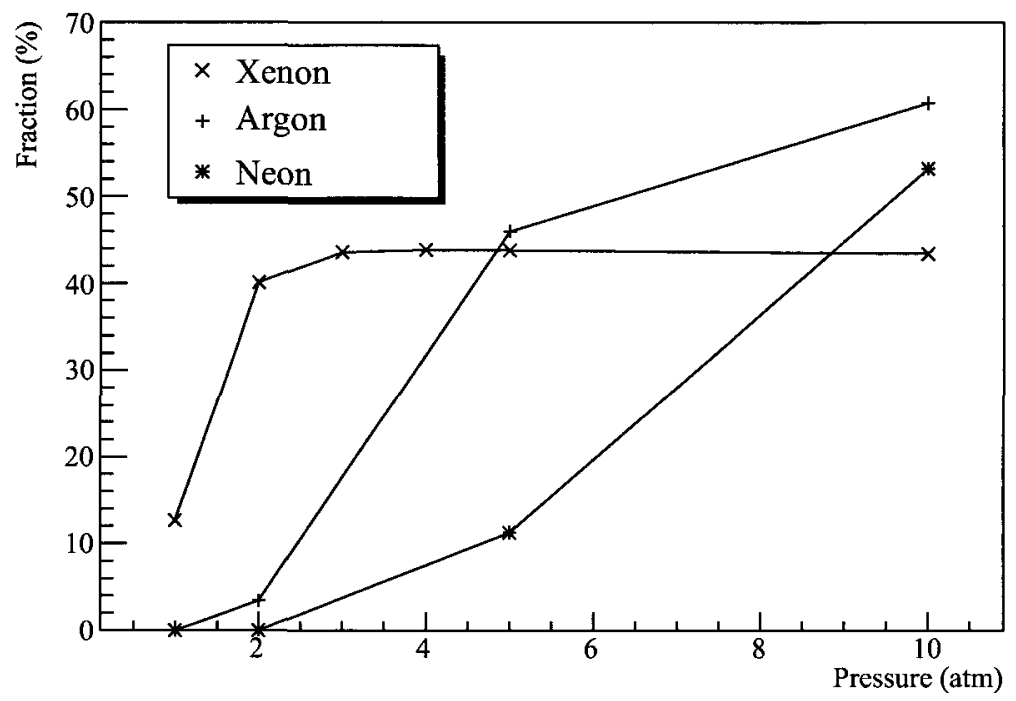

(a)

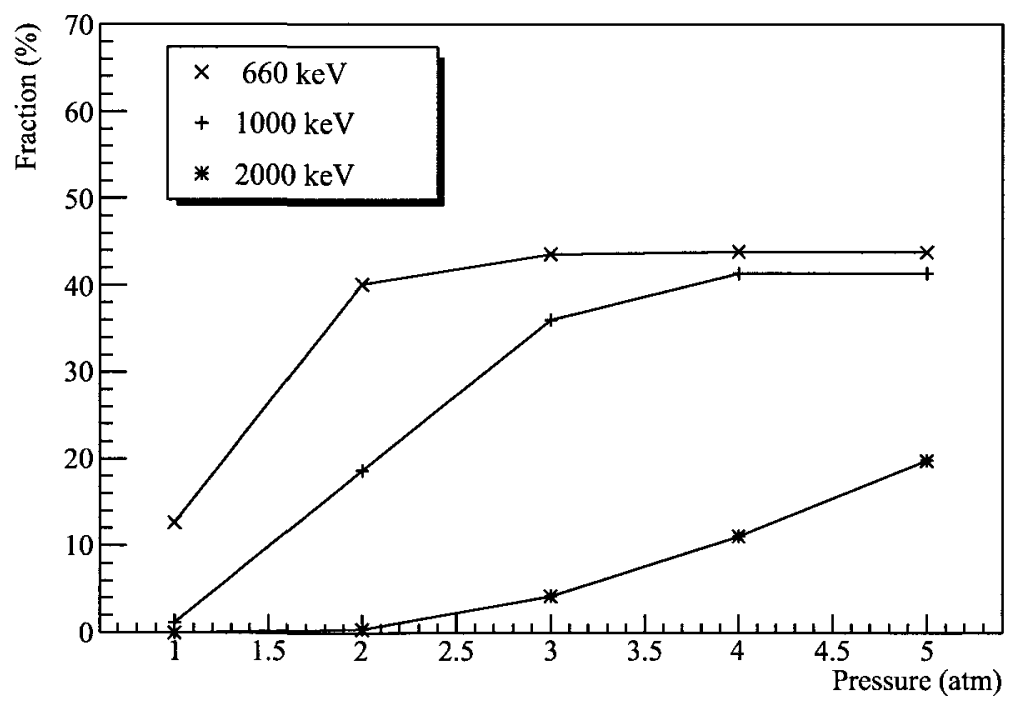

(b)

Figure 3.8: Fraction of events having $99 \%$ of their energy contained as a function of pressure for different electron energies and gas types. An electron energy of $660 \mathrm{keV}$ was used for (a), while for (b) the gas used was pure xenon. 


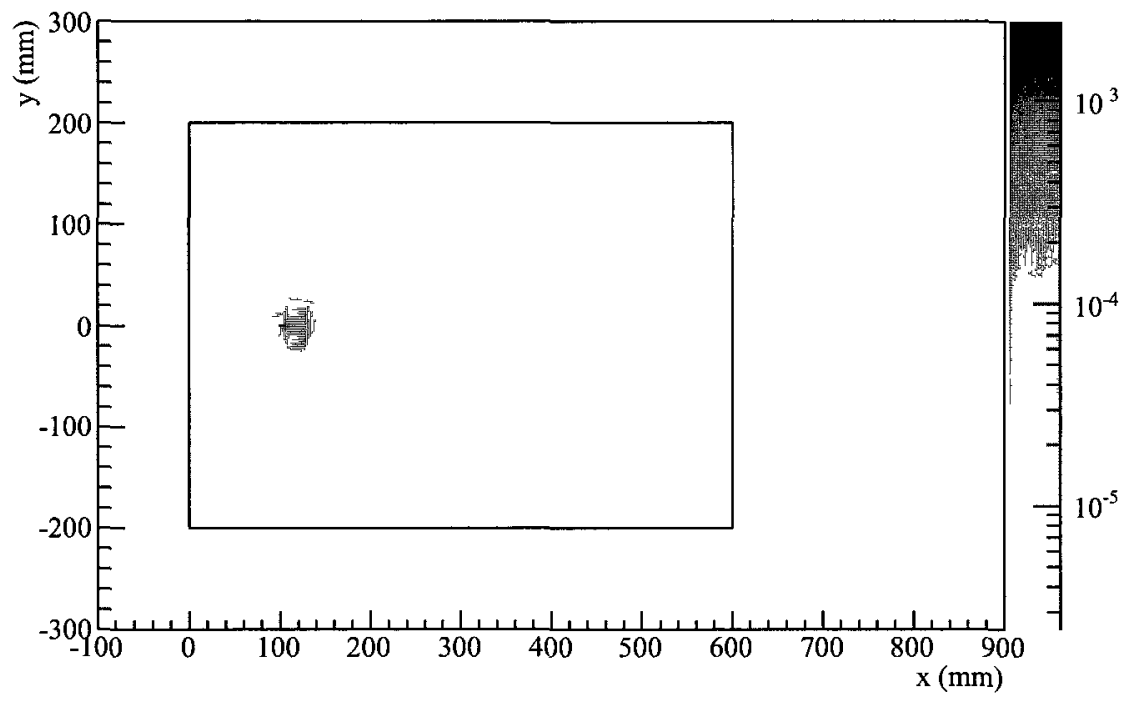

(a)

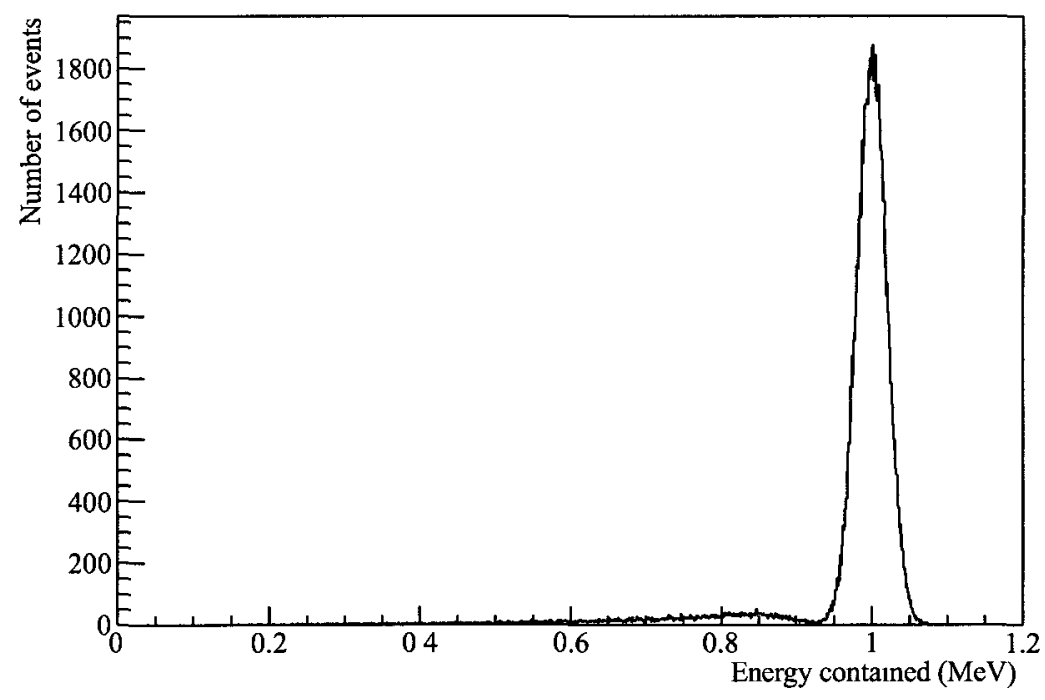

(b)

Figure 3.9: Track density (a) and reconstructed energy (b) for $1 \mathrm{MeV}$ electrons in $10 \mathrm{~atm}$ xenon. The source is shifted $100 \mathrm{~mm}$ toward the centre of the detector. Note the increased number of contained events compared to Figure 3.7. 
$2 \nu \beta \beta$ decay signal. The fraction of events with one or more gammas having more than $10 \mathrm{keV}$ leaving the volume is shown in Figure 3.11 for different gases. A larger gas mass means a larger number of gamma rays created. This effect is expected since the radiation to ionization energy loss ratio depends linearly on the number of protons in the atom. Again, it would be advantageous to mix xenon with neon or argon to limit the number of gamma rays produced. 


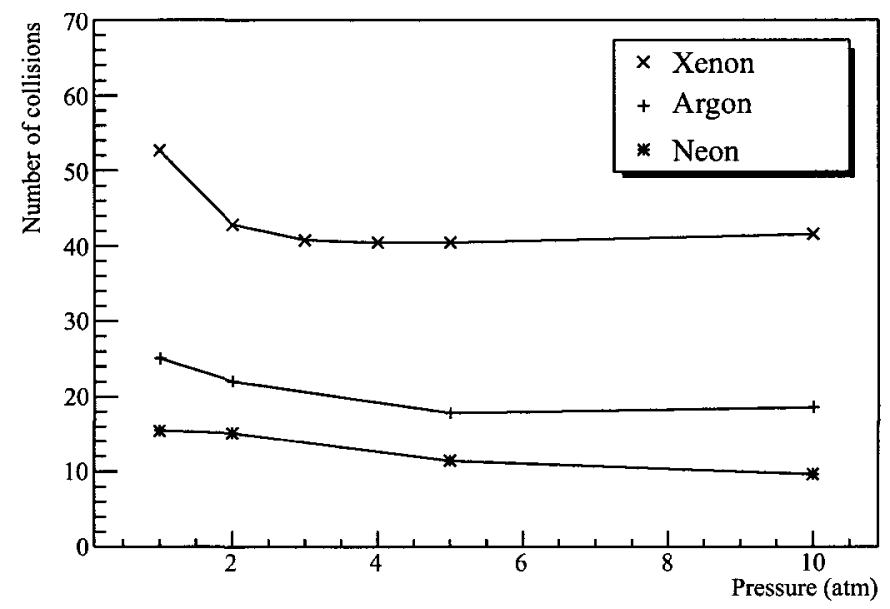

Figure 3.10: Number of large angle collisions per event as a function of pressure for $660 \mathrm{keV}$ electrons and different gas types. A large angle collision occurs when the trajectory changes by more than 45 degrees and if the energy of the primary electron is higher than $10 \mathrm{keV}$.

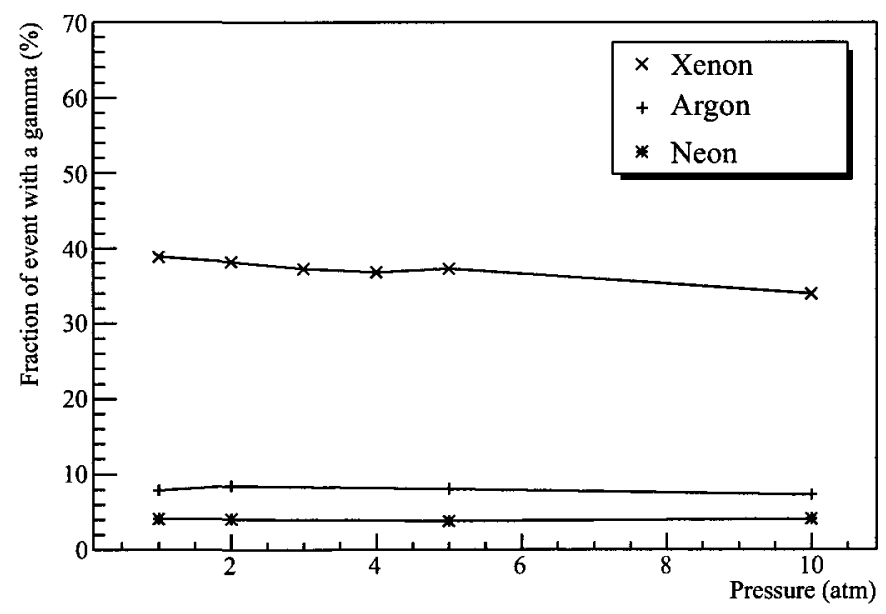

Figure 3.11: Fraction of events with a gamma having an energy greater than $10 \mathrm{keV}$ leaving the detector volume as a function of pressure for $660 \mathrm{keV}$ electrons and different gas types. 


\section{Chapter 4}

\section{Barium Ion Laser Induced}

\section{Fluorescence}

A measurement of $\left\langle m_{\beta \beta}\right\rangle$ from $0 \nu \beta \beta$ decay requires a very precise control of the background events. An experiment needs to carefully select and clean its material to limit the radioactivity in the vicinity of the sensitive volume. It also needs to be shielded from the natural radioactivity of the environment. Finally, it needs be buried underground to reduce the amount of cosmogenic radioactivity. Unfortunately, due to the very low signal rate of $0 \nu \beta \beta$ decay, extreme brackground reduction efforts are required in order to produce a convincing measurement.

The identification (tagging) of the barium daughter in the $\beta \beta$ decay of xenon would dramatically suppress the background events and Laser Induced Fluorescence (LIF) has been studied for this purpose. This chapter will describe the theory of LIF applied to barium ions in vacuum and in gas. Then, it will describe the setup used for the fluorescence study. Finally, two techniques to produce barium ions will be described, one using an electrical arc and the second using pulsed laser light, with the details of the two associated fluorescence chambers built to investigate these tagging techniques.

During the research program, it was decided that tagging in high pressure gas 
is not the most efficient technique. It might be more effective to extract ions into a trap before performing the tagging. Nevertheless, this chapter shows the successes and failures of producing and detecting $\mathrm{Ba}^{+}$in both vacuum and gas.

\subsection{Laser Induced Fluorescence Theory}

LIF is a very popular spectroscopy technique and is used in many applications. It is performed by aiming a laser at a medium. If the wavelength of the laser is tuned to one of the resonances of the medium, fluorescence will occur and an analysis of its content can be done. This technique has many advantages for barium tagging. First, the analysis is non-destructive. Second, it can be done remotely if required. Third, the identification of any atom or molecule is made very accurately since two species having the exact same resonance energy is improbable. Fourth, the identification of the position or the distribution in space can be done since the scattered photons are isotropic. Fifth, the analysis is done in real time, which allows a measurement of a reaction rate, or to signal the occurrence of an event. Finally, if the atom is placed in a trap, the sensitivity has been demonstrated to be at the single atom level [43], which is what is required for barium tagging.

\subsubsection{Ionic Species of Barium}

Although the $\beta \beta$ decay of xenon produces $\mathrm{Ba}^{++}$, it would be nearly impossible to detect this ion via LIF. $\mathrm{Ba}^{++}$is basically a $\mathrm{Ba}$ nucleus with a very tight $\mathrm{Xe}$ electron shell around it which requires a far UV light to excite. The first ionization energy of $\mathrm{Xe}$ is $12.13 \mathrm{eV}$ which is larger than the electron affinity of $\mathrm{Ba}^{++}$of $10.00 \mathrm{eV}$, so the rate of electron transfer should be relatively slow in pure xenon, unless the barium ion is given a high kinetic energy. Various molecules can be used as electron donors. For example, $\mathrm{Ba}^{++}$in triethylamine (TEA) should have a fast electron capture rate since the TEA ionization potential is much smaller at 
$7.5 \mathrm{eV}$ than the $\mathrm{Ba}^{++}$electron affinity. Once the $\mathrm{Ba}$ ion is in the first ionized state, its electron affinity becomes $5.21 \mathrm{eV}$, much lower than any other molecules in the volume. Therefore, the $\mathrm{Ba}^{+}$should stay in this state for a long period of time. Any electron donor additive to the xenon gas will likely reduce or even cancel all the EL signal required for an energy measurement. Therefore, the most recent concept for a gas time projection chamber (TPC) with barium tagging allows the $\mathrm{Ba}^{++}$to be extracted from the xenon volume through a nozzle. The ion will then be allowed to acquire an electron from a low ionization potential gas.

One could also wonder about tagging the neutral barium atom. Indeed, it is possible to neutralize the barium ion and excite its very fast and strong transition between the ground state and the 6s6p state. But there are three problems, one is the decay of the excited state which can go back to the ground state, but also to three other states which are metastable. The second problem is the control over the atom once it is neutralized. In the ionized state, an electric field can be used to force it to drift through a region to perform the tagging. The third problem is the accumulation of all the barium atoms in the volume. Every time a $2 \nu \beta \beta$ decay would occur, a barium atom would wander in the volume that could be wrongly tagged after a $0 \nu \beta \beta$ decay. If the ion is tagged instead of the neutral atom, it is possible to drift it out of the tagging volume until it gets neutralized. That is why tagging the $\mathrm{Ba}^{+}$is the only option considered in this study.

\subsubsection{Energy Levels of $\mathrm{Ba}^{+}$}

The spectroscopy of $\mathrm{Ba}^{+}$is well known since it behaves more or less like an alkali atom, having only one electron outside a closed shell making the energy levels easy to calculate. Its energy levels are shown in Figure 4.1. $\mathrm{Ba}^{+}$has been studied in depth as early as 1975 by Dehmelt [44] since the $6^{2} S_{1 / 2}-5^{2} D_{3 / 2}$ transition was expected to be one of the sharpest lines known. In 1978, the same group achieved a very important milestone by being able to detect a group of 10 to 20 ions visually, 


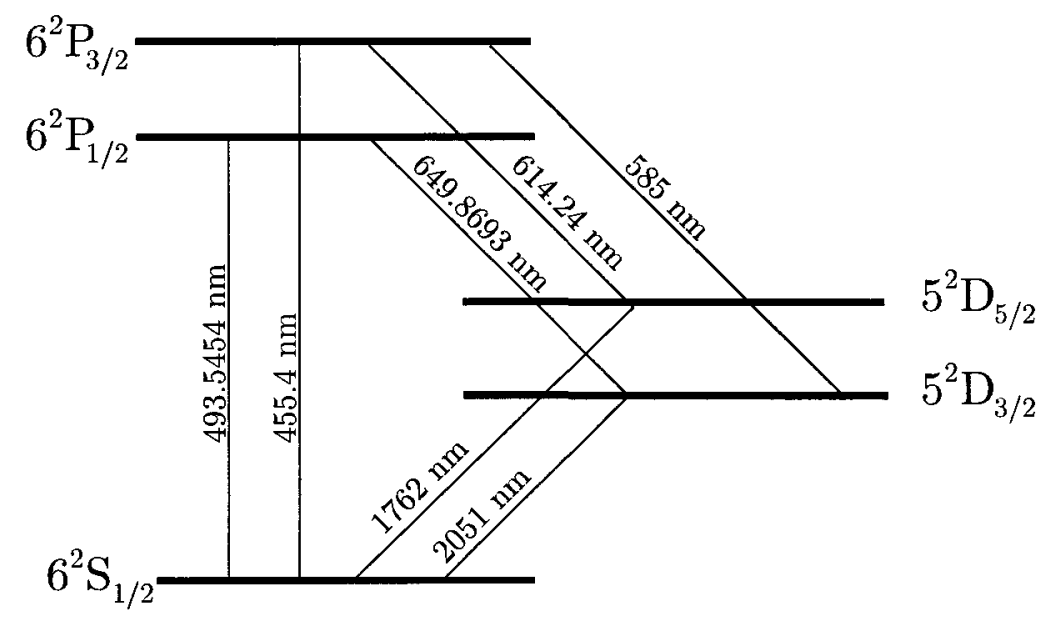

Figure 4.1: Energy levels of the barium ion and the wavelength in vacuum for the different transitions. Values taken from Curry et al. [47].

photographically and photoelectrically [45]. It took only two years to refine their technique to localize single barium ions [46].

Later, between 1978 and 1982, barium spectroscopy was used to understand the nuclear structure of the atom by measuring the resonance line shift for different isotopes which translates into a nucleus radius. With one of the longest metastable states known, the $5^{2} D_{3 / 2}$ state of $\mathrm{Ba}^{+}$, scientists had high expectations of using this to increase the accuracy of atomic clocks.

\subsubsection{Different Procedures Investigated}

Many procedures are described in the EXO proposal paper of 1999 [41]. Below is a list of four of them, with the addition of a new technique investigated by this research. Some of them are not sensitive enough, others are not practical and some are not possible in high pressure gas. 


\section{Continuous Excitation}

Most of the spectroscopic measurements of $\mathrm{Ba}^{+}$were done in vacuum with helium as buffer gas at a level around $10^{-5}$ Torr. In that case the procedure is simple. A $493 \mathrm{~nm}$ laser is used to excite the ground state to the first excited state $\left(6^{2} S_{1 / 2}-6^{2} P_{1 / 2}\right)$. Then, the decay back to the ground state takes $7.74 \pm 0.4 \mathrm{~ns}$ [48] and the fluorescence photon can be detected. By saturating this transition, about $10^{8}$ photons per second can be produced. Unfortunately, $26.5 \pm 2 \%$ of the time [48] the excited state decays into the metastable state $\left(6^{2} P_{1 / 2}-5^{2} D_{3 / 2}\right)$, which stops the fluorescence. Therefore, a second laser at $650 \mathrm{~nm}$ is used to unshelve the ion from the $D$ state back to the $P$ state. Both lasers are run continuously. This procedure maximizes the number of photons produced and is very simple to implement. On the other hand, this technique becomes useless at higher pressures because of Rayleigh scattering. When the pressure of the gas increases, the number of photons scattered increases as well. Following the theory in Jackson's book [49], the attenuation length of a photon in a gas can be calculated with:

$$
\alpha \simeq \frac{4(2 \pi)^{3}}{3 N \lambda^{4}}|n-1|^{2}
$$

where $\alpha$ is the attenuation length, $\lambda$ the wavelength, $n$ the refractive index and $N$ the number density. At $1 \mathrm{~atm}$ and $23^{\circ} \mathrm{C}$, the number density of xenon is:

$$
N=\frac{P}{k_{B} T}=2.48 \times 10^{25} \mathrm{~m}^{-3}
$$

With $n=1.0008,1 / \alpha$ becomes $6810 \mathrm{~m}$. Therefore, over a distance of $0.05 \mathrm{~m}$, $1-e^{-\alpha x}=7.3 \times 10^{-6}$ of the photons will be scattered. At $493 \mathrm{~nm}$, the energy carried per photon is $E=h c / \lambda=4.03 \times 10^{-19} \mathrm{~J}$, where $h$ is the Planck constant and $c$ the speed of light. For $100 \mathrm{~mW}$ of power, the laser beam carries $0.1 \mathrm{~J} \mathrm{~s}^{-1}$, or $2.48 \times 10^{17}$ photons per second. Therefore, $1.8 \times 10^{12}$ photons will be scattered per second. If the observation is made perpendicular to the beam and in the direction 
of the polarization of the beam, no Rayleigh scattering is expected to be observed directly. However, even with a chamber wall coated to absorb $99 \%$ of the scattered light and a PMT with a $2 \%$ solid angle, $3.6 \times 10^{8}$ photons per second scattered will hit the photomultiplier tube (PMT) which is still higher than the fluorescence signal from the ion.

Rayleigh scattering is not the only process that can create background photons. Any other scattering process, reflections from apertures and windows, Mie scattering from larger particles, or even diffraction from parts of the pressure vessel would create photons that would be detected the same way as those coming from the ion.

To add to the problem, as the pressure in the chamber increases, the linewidth of the transition increases. Therefore, one is required to increase the laser power to reach saturation, increasing both Rayleigh and other type of scattering. Other types of tagging techniques are more adapted to tagging in high pressure gas.

\section{Frequency Modulation}

This technique is a variation of the continuous excitation. To increase the sensitivity of the test, it has been proposed to use an electro-optic modulator (EOM) to change the frequency of the blue light, away from resonance, but keeping the same lasing power. Both the blue and the red lasers would be on, but modulation of the frequency of the blue laser would translate into a modulation in the count rate. The broadening of the line due to pressure makes this technique impractical in gas. The shift of wavelength would be too large. This technique has not been considered in this study.

\section{High Speed Excitation}

This technique requires a very fast pulsed laser system. If the $493 \mathrm{~nm}$ laser is pulsed with a duration in the picosecond regime, one would be able to quickly excite the $S$ state to the $P$ state and observe the decay of it. The time distribution 
of those photons would be a exponential decay with a lifetime of 8 ns. Since the $493 \mathrm{~nm}$ laser would be off during the measurement period, Rayleigh scattering would be limited to late, multiply reflected light and possibly not an issue. The procedure could be repeated every 25-50 ns. One possible problem to consider is the relaxation time of the PMT. While the blue laser is on, many photons are detected by the PMT, heating it, making it noisier and easier to trigger. If the current in the PMT is high enough, the noise rate can increase significantly and fake counts can be included in the signal. This technique has never been considered in this study.

\section{Intermodulation}

This fourth technique exploits the fact that the ion can be shelved for a very long period of time in the $D$ state $(89.4 \pm 15.6 \mathrm{~s}$ in vacuum [50]). By exciting the $493 \mathrm{~nm}$ transition $n$ times, one knows with a $1-\left(\Gamma_{P S}\right)^{n}$ probability that the ion will be in the $D$ state, $\Gamma_{P S}=26.5 \%$ being the branching ratio from the $P$ to the $S$ state. Then, one can turn off the $493 \mathrm{~nm}$ laser, turn on the $650 \mathrm{~nm}$ laser to unshelve the ion from the $D$ to the $P$ state and observe the $493 \mathrm{~nm}$ photon from the decay to the ground state. An optical filter placed over the PMT filters out photons outside a narrow window around $493 \mathrm{~nm}$. Consequently, $650 \mathrm{~nm}$ photons are not detected and, since the $493 \mathrm{~nm}$ laser is off during this period, the signal comes only from the ion.

The same problem as with the previous technique can occur. While the blue laser is on, the PMT heats up and is noisier. This problem has an easy solution. The PMT can be given a few $\mu$ s to cool down, but this would slow down the whole procedure. The laser light could be controlled with a set of two Acousto-Optic Modulators (AOM) arranged in series to steer the beam through a slit when light is required. A typical tagging protocol could be the following. The $493 \mathrm{~nm}$ laser would be on for a period of $5 \mu \mathrm{s}$, then turned off for the next $5 \mu \mathrm{s}$ to let the PMT cool down. Then, the $650 \mathrm{~nm}$ laser would be turned on for another $5 \mu$ s and blue 
photons would be counted during this period. After the $650 \mathrm{~nm}$ laser would be turned off, the $493 \mathrm{~nm}$ laser would start again for another $15 \mu$ s cycle.

This technique has been considered early in the research program, although no fluorescence has ever been measured that way. The problem is likely due to the depopulation of the $D$ state in gas. Collisions with the neighbouring atoms can induce a preemptive radiationless decay of the $D$ state. After a careful review of the literature a new technique, called pressure depopulation tagging, has been developed that makes use of the depopulation phenomenon.

\section{Pressure Depopulation}

A high gas pressure would increase the number of collisions and therefore increase the probability of depopulating the $D$ state. The LIF technique requires the $493 \mathrm{~nm}$ laser to excite the ion. Then, $30 \%$ of the time a red photon would be observed and instead of deshelving the ion with a laser light, the ion would go back to the ground state, giving its energy by colliding with the surrounding gas. Ruster et al. [51] were the first to measure fluorescence from $\mathrm{Ba}^{+}$using this technique, using instead the $455 \mathrm{~nm}\left(P_{3 / 2}-S_{1 / 2}\right)$ transition. They used $\mathrm{Ba}^{+}$in a Paul trap (radio frequency quadrupole trap) at modest pressures of hydrogen as the buffer and quencher gas. In order to contain ions in the trap, a low pressure of a light gas is required. Since molecular hydrogen has a depopulation cross-section many orders of magnitude larger than noble gases, it was the appropriate choice for them. One can assume that it is possible to use a noble quencher gas at much higher pressures.

Madej et al. [52] measured a quenching rate for a combination of the $D_{3 / 2}$ and $D_{5 / 2}$ states of $\mathrm{Ba}^{+}$in argon 52.5 times lower than for $\mathrm{H}_{2}$. Extrapolating toward higher pressures, around $1 \mathrm{~atm}$, the $D$ state becomes unstable with a lifetime of a few ns. Such a short lifetime would limit the efficiency of the intermodulation procedure since the $D$ state would decay into the ground state by energy transfer to neighbouring atoms. Fortunately, the pressure depopulation tagging procedure 
can become almost as efficient as the continuous excitation technique if the tagging is done at the right pressure. Using only the $493 \mathrm{~nm}$ laser, it is possible to saturate the $6^{2} S_{1 / 2}-6^{2} P_{1 / 2}$ and $26.5 \%$ of the time the $P$ state decays into the $D$ state emitting a $650 \mathrm{~nm}$ photon that is detected. Later, the $D$ state decays into the ground state that is ready to be excited again. This way, the whole cycle takes about 100 ns and by using a filter to absorb blue photons, it is possible to have a background free measurement.

Unfortunately, tagging has not been demonstrated using this technique. Inefficiencies, a lack of a reliable $\mathrm{Ba}^{+}$source at high pressures and repetitive instrument breakdowns plagued the study.

\subsubsection{Classical Ion-Atom Collision Theory}

As discussed previously, the gas pressure modifies the lifetime of the $D$ state. It is possible that, because of collisions, the energy is no longer emitted via the E2 radiative transition, but is transfered to neighbours. The collision rate and the quenching ratio are the two quantities needed to calculate the lifetime.

The collision rate is the number of times the ion enters a region around a polarized particle where the ion is considered captured. To go through the algebra, books by Bowers [53] and McDaniel [54] are very helpful. As the ion approaches an atom, the electron cloud is pulled toward the ion, an effective charge appears, attracts the ion and possibly captures it. At close distances, the force will become repulsive and the ion will be repelled from the atom.

\section{Classical Ion-Dipole Potential}

Taking the atom as a spherical capacitor with a medium of electric permittivity of $\chi$, the dipole moment $\mu$ can be written as a function of the electric field $E$ and the density of atoms $N$ as 


$$
\mu=\frac{\chi E}{4 \pi N} .
$$

Knowing that the force $F$ of a dipole on a charge $q$ over a distance $r$ is

$$
F=\frac{2 \mu q}{r^{3}} \cos \beta
$$

with the angle between the dipole moment and the direction to the charge $q$ being $\beta$, the force on the ion as a function of distance is calculated, allowing the calculation of the potential created by the system.

Since the dipole is induced by the ion, the angle $\beta$ will always be 0 , and if the electric field $E$ is substituted by the known Coulomb field, $E=e / r^{2}$, the force becomes

$$
F=\frac{\chi q^{2}}{2 \pi N r^{5}}
$$

Then, the potential energy of the system is obtained by integrating the force,

$$
V(r)=-\int_{r}^{\infty} \frac{\chi q^{2}}{2 \pi N r^{5}} d r
$$

or,

$$
V(r)=-\frac{\chi q^{2}}{8 \pi N r^{4}}
$$

It is possible to write the potential as a function of the polarizability $\alpha$, instead of the susceptibility, with $\alpha=\frac{\chi}{4 \pi N}$ :

$$
V(r)=-\frac{\alpha q^{2}}{2 r^{4}} .
$$

It is very important to note that this potential is the dipole approximation and if one wants to be more precise, the quadrupole moment and higher orders have to 


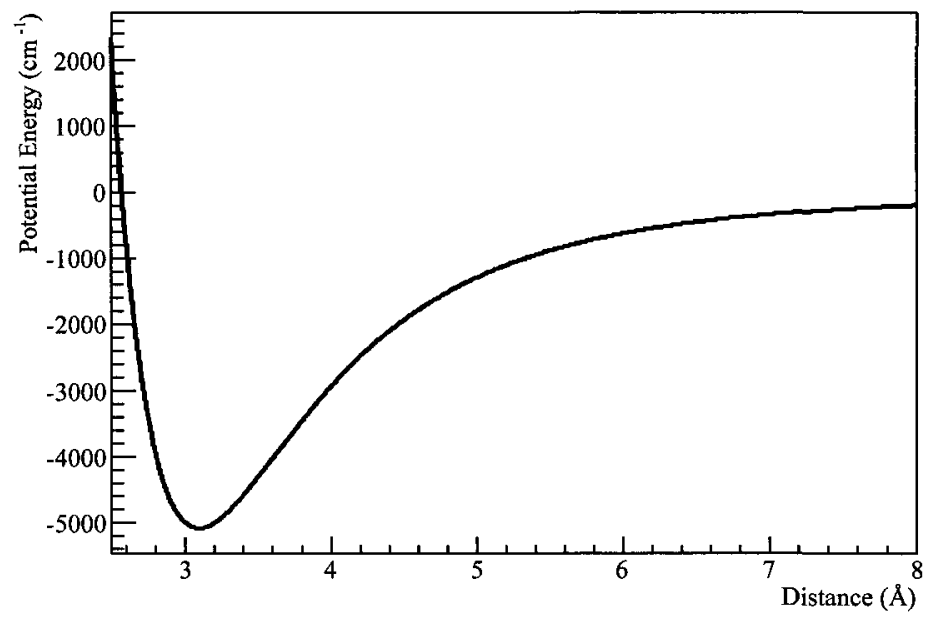

Figure 4.2: Potential energy shape of $\mathrm{Ba}^{+}$and Ar. Note the binding energy of $5000 \mathrm{~cm}^{-1}(\simeq 0.6 \mathrm{eV})$ at a distance of $3.1 \AA$.

be included. Also, if the neighbouring molecules have a permanent dipole moment, the potential is different and needs to be calculated with one of the many average dipole orientation (ADO) theories.

Equation 4.8 is the main attraction potential at large distances, but at close distances a repulsive term becomes dominant taking the form of:

$$
V_{R}(r)=+A e^{-b r}
$$

with $A$ and $b$ two empirical constants. Bellert and Breckenridge [55] summarize these values for many ions and rare gases, and give $A=4.57 \times 10^{7} \mathrm{~cm}^{-1}$ and $b=3.032 \AA^{-1}$ for $\mathrm{Ba}^{+}$and Ar. Combining Equations 4.8 and 4.9 and inserting the calculated values for $\mathrm{Ba}^{+}$and $\mathrm{Ar}$ gives the potential shape depicted in Figure 4.2.

The two-body mechanics that follows is quite simple and will be reviewed quickly. In polar coordinates, the kinetic energy in the centre of mass frame $\left(K_{C M}\right)$ of a two-body system is: 


$$
K_{C M}=\frac{1}{2} \mu\left(\dot{r}^{2}+r^{2} \dot{\phi}\right)
$$

with $r$ the distance between the particles, $\mu$ their reduced mass and $\phi$ the angle in the plane. When interactions are small $(r \rightarrow \infty)$ the energy $(E)$ of the system should be kinetic only, but later the energy is the sum of the kinetic energy and the potential energy. And since energy is conserved in the process:

$$
E=\frac{1}{2} \mu v_{0}^{2}=\frac{1}{2} \mu\left(\dot{r}^{2}+r^{2} \dot{\phi}\right)+V(r),
$$

with $v_{0}$ being the initial velocity.

A similar reasoning can be applied to the angular momentum in the centre of mass $\left(L_{C M}\right)$ with the impact parameter $b$, at large distance and along the path, which are equal by conservation of angular momentum.

$$
L_{C M}=\mu b v_{0}=\mu r^{2} \dot{\phi}
$$

If the $\dot{\phi}$ in the Equation 4.11 is replaced by $b v_{o} / r^{2}$ from Equation 4.12 , the relation becomes a one dimension one,

$$
\frac{1}{2} \mu v_{0}^{2}=\frac{1}{2} \mu \dot{r}^{2}+\frac{\mu b^{2} v_{0}^{2}}{2 r^{2}}+V(r) .
$$

The term in $1 / r^{2}$ can be considered as a centrifugal potential, appearing when the dependence of the angle $\phi$ is removed. Then the closest point between the ion and the atom is found by minimizing $r$ :

$$
\frac{d r}{d \phi}=\frac{d r}{d t} / \frac{d t}{d \phi}= \pm \frac{r^{2}}{b}\left[1+\frac{\alpha q^{2}}{\mu v_{0}^{2} r^{4}}-\frac{b^{2}}{r^{2}}\right]^{1 / 2}=0 .
$$

The only non-trivial way to make Equation 4.14 a true statement is to have the square bracket expression equal to zero, which has a solution for: 


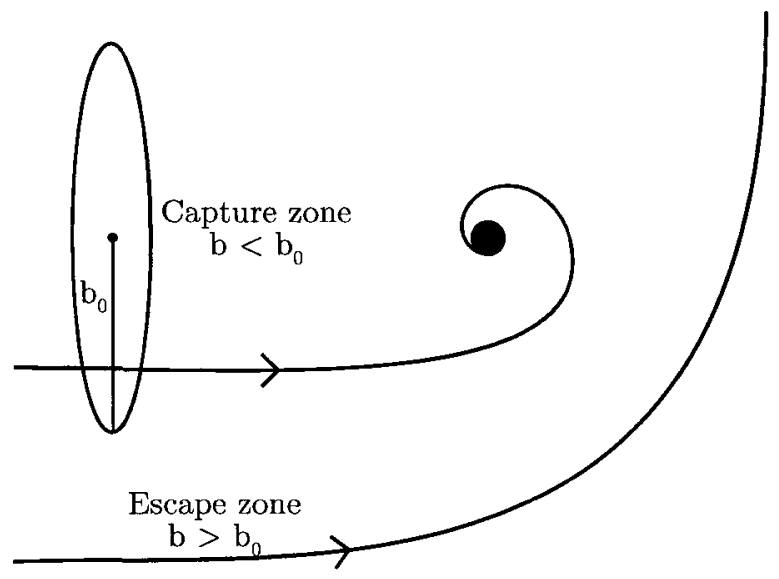

Figure 4.3: For a given $v_{0}$, the cross-section can be calculated by the area of the circle through which all captured ions go through.

$$
b_{0}^{2}=\sqrt{\frac{4 q^{2} \alpha}{\mu v_{0}^{2}}} .
$$

This means that every particle going through a circle of radius $b_{0}$ will be either captured by the dipole or collide if a repulsive force exists at small distances. Figure 4.3 illustrates this example. Therefore, the cross-section of a collision between an ion of charge $q=1$ and a non-polar atom is:

$$
\sigma\left(v_{0}\right)=\pi b_{0}^{2}=2 \pi \sqrt{\frac{\alpha}{\mu v_{0}^{2}}}
$$

Finally, the rate constant $k$ is:

$$
k=2 \pi \sqrt{\frac{\alpha}{\mu}}
$$

which is independent of the velocity. The collision rate will be constant regardless of the distribution of the ion. Therefore, one can use the rate constant measured near vacuum in a trap and apply it in atmospheric pressure gas. 
According to the last equation, the rate constant for a barium ion in xenon gas is $5.787 \times 10^{-16} \mathrm{~m}^{3} / \mathrm{s}$. The density $N$ of xenon at 1 atm at $23^{\circ} \mathrm{C}$ is $2.48 \times$ $10^{25}$ atoms $/ \mathrm{m}^{3}$, which translates into a collision rate $\left(R_{\text {coll }}\right)$ of:

$$
R_{\text {coll }}=k N=1.43 \times 10^{10} \mathrm{~s}^{-1} .
$$

It is worthwhile to note that, using a hard sphere collision model as described in McDaniel's book [54], one obtains a collision rate of $5.8 \times 10^{9} \mathrm{~s}^{-1}$, which is a factor 2 too small, showing the influence of the attractive potential of the induced dipole in Xe.

\subsubsection{Quenching Ratio}

The theory developed in Section 4.1.4 tells nothing about the probability of transferring energy from the ion to the atom or the inverse. The quenching ratio is the probability that a collision will lead to a decay of the excited state to the ground state. According to Madej and Sankey [52], this ratio for the $D_{5 / 2}$ of $\mathrm{Ba}^{+}$is around 0.3 for simple molecules $\left(\mathrm{H}_{2} \mathrm{O}, \mathrm{CO}\right.$ or $\left.\mathrm{N}_{2}\right)$, and can go as high as 0.6 for more complex molecules like $\mathrm{CH}_{4}$. On the other hand, for a noble gas like $\mathrm{Ar}$, the ratio is very low. By using an indirect measurement, they estimate the quenching rate to be $1600 \pm 1300 \mathrm{~s}^{-1} \mathrm{~Pa}^{-1}$ or a quenching ratio of $0.010 \pm 0.007$. This can be explained by the high energy needed to excite Ar, which is around $14 \mathrm{eV}$. For Xe, the energy is about $10 \mathrm{eV}$, therefore the 0.01 ratio should be regarded as a lower limit. Those ratios were calculated from data taken in a trap with a larger kinetic energy than in high pressure gas. But according to Madej [56], it would not be surprising if the quenching rate was in the $\mathrm{ns}^{-1}$ regime at atmospheric pressure. He also noted that, as the pressure increases, the quenching ratio should also increase since the number of three body collisions becomes important. A three body collision would offer more degrees of freedom for the distribution of the energy of the ion. Figure 4.4 shows the lifetime of the $D_{3 / 2}$ state of $\mathrm{Ba}^{+}$in $\mathrm{Ar}, \mathrm{Ne}$ and $\mathrm{He}$ as a function 


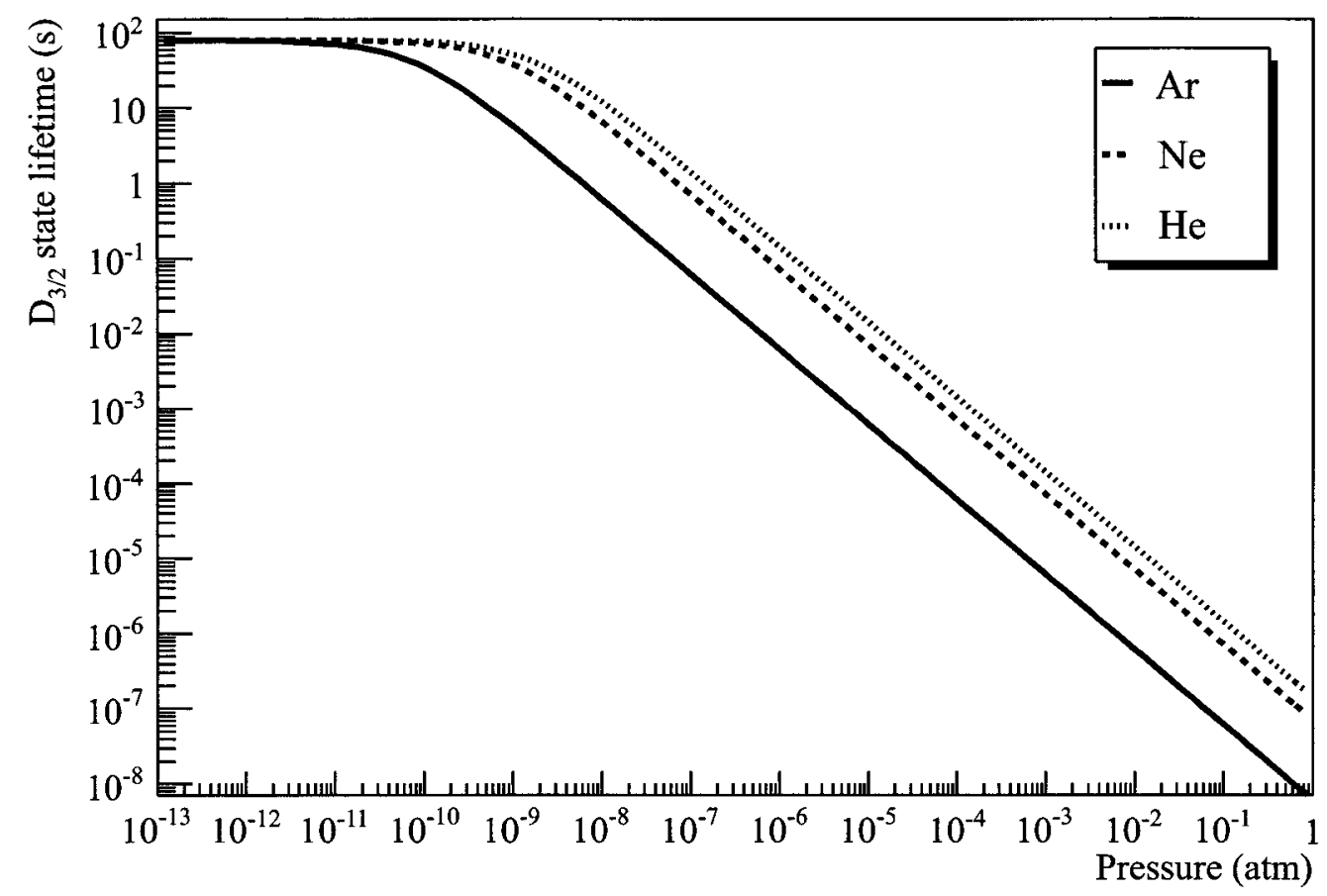

Figure 4.4: Calculated quenching rate of barium ions in $\mathrm{Ar}, \mathrm{Ne}$ and $\mathrm{He}$ as a function of pressure, using the experimental measurements of Madej and Sankey [52] and Hermanni and Werth [57].

of pressure, assuming it is the same as the $D_{5 / 2}$ state, an assumption supported by Madej and Sankey [52] and Hermanni and Werth [57], from whom the data has been taken. All measurements were made at very low pressure, between $10^{-11}$ and $10^{-6} \mathrm{~atm}$, so a large part of the curve is an extrapolation. The three body quenching enhancement is not taken into account.

\subsubsection{Linewidth}

One of the drawbacks of tagging the ion in gas is the pressure broadening of the transition. As the pressure increases, the potential between molecules is different 
and the energy levels change. For this reason, the intensity needed to saturate the transition needs to be increased to balance the lower cross-section. Pressure is not the only factor that broadens the line and other processes will be reviewed in this section. A more complete development can be found in Demtröder's book [58].

\section{Natural Linewidth}

The natural linewidth can be calculated easily using the uncertainty principle but the classical calculation is more descriptive and will help to understand other types of broadening. The natural width of a transition is usually small and can be neglected most of the time.

An excited atom can be described by a weakly damped harmonic oscillator with its amplitude expressed as:

$$
\ddot{x}+\gamma \dot{x}+\omega_{0}^{2} x=0,
$$

with the solution for a very small damping $\gamma$ being:

$$
x(t)=x_{0} e^{(\gamma / 2) t} \cos \omega t .
$$

$x_{0}$ is the position at $t=0$ and $\omega=\sqrt{\omega_{0}^{2}-\gamma^{2} / 4} \simeq \omega_{0}$

The Fourier transform of the last equation gives the amplitude distribution as a function of frequency:

$$
A(\omega)=1 / \sqrt{2 \pi} \int_{-\infty}^{\infty} x(t) e^{-\imath \omega t} d t
$$

or

$$
A(\omega)=x_{0} / \sqrt{8 \pi}\left(\frac{1}{i\left(\omega-\omega_{0}\right)+\gamma / 2}+\frac{1}{i\left(\omega+\omega_{0}\right)+\gamma / 2}\right) .
$$

Then, taking the intensity $I(\omega)=A(\omega) A^{*}(\omega)$ and normalizing, it becomes: 


$$
I(\omega)=\frac{1}{2 \pi} \frac{\gamma}{\left(\omega-\omega_{0}\right)^{2}+(\gamma / 2)^{2}},
$$

which is a Lorentzian distribution. The FWHM is equal to $\delta \omega=\gamma$, or using the lifetime of the decay $\tau=1 / \gamma$ and the frequency $\nu=\omega / 2 \pi, \delta \nu=1 / 2 \pi \tau$. Therefore, the $6^{2} P_{1 / 2}$ transition to the ground state with a lifetime of $7.7 \mathrm{~ns}$ has a natural width of $20.67 \mathrm{MHz}$.

\section{Doppler Broadening}

Another source of broadening is the Doppler shift of the line. At equilibrium the ion will have a velocity along the direction perpendicular to the observer following a Maxwell distribution:

$$
P\left(v_{z}\right) d v_{z}=\sqrt{\frac{m}{2 \pi k_{B} T}} e^{m v_{z}^{2} / 2 k_{B} T} d v_{z} .
$$

Doppler's law is written as:

$$
\omega=\omega_{0}\left(1+v_{z} / c\right),
$$

which says that a shift $d v_{z}$ in velocity will result in a shift in frequency of $d \omega c / \omega_{0}$. Therefore, Equation 4.24 becomes:

$$
P(\omega) d \omega=\sqrt{\frac{m c^{2}}{2 \pi \omega_{0} k_{B} T}} e^{m c^{2} / 2 k_{B} T\left(\frac{\omega-\omega_{0}}{\omega_{0}}\right)^{2}} d \omega
$$

which is a Gaussian distribution. Once normalized, the FWHM becomes

$$
\delta \omega=\left(\omega_{0} / c\right) \sqrt{8 \ln 2 k_{B} T / m},
$$

or 


$$
\delta \nu=7.16 \times 10^{-7} \nu_{0} \sqrt{T / M}
$$

For a barium ion at room temperature, the $6^{2} P_{1 / 2}$ transition to the ground state should be $639.66 \mathrm{MHz}$ wide.

\section{Collision Broadening}

Also referred to as pressure broadening, collision broadening involves a modification of the potential due to the neighbouring atoms. Atoms with a strong polarization will tend to induce a larger modification to the linewidth. This is difficult to calculate and easier to measure. Zokai et al. [59] have measured the pressure broadening for the $P_{1 / 2}-S_{1 / 2}$ line at $493 \mathrm{~nm}$ of $\mathrm{Ba}^{+}$in $\mathrm{Ar}$ and He. Using a flow lamp and a high resolution pressure-scanned etalon they measured the width of the line between number densities of about 0.2 to $7.2 \times 10^{24} \mathrm{~m}^{-3}$ at $1300 \mathrm{~K}$ (between about 36 to $130 \mathrm{mbar}$ ). They measure a broadening of $1.89(9) \times 10^{-26} \mathrm{~cm}^{-1} \mathrm{~m}^{-3}$ in $\mathrm{Ar}$ and $1.31(6) \times 10^{-26} \mathrm{~cm}^{-1} \mathrm{~m}^{-3}$ in He. Therefore, at atmospheric pressure, the linewidth of the $493 \mathrm{~nm}$ transition should be $15 \mathrm{GHz}$, or a broadening of $19 \mathrm{MHz} /$ Torr.

\subsection{Laser Induced Fluorescence Apparatus}

As noted in Section 4.1.3, two lasers are required to induce continuous fluorescence in the first two levels of the barium ion. To probe the two interesting transitions, the $S_{1 / 2}-P_{1 / 2}$ and the $P_{1 / 2}-D_{3 / 2}$, dye lasers were selected for their ability to provide a wavelength with a high accuracy over a broad range of wavelength. Both of them are Coherent 899-01 ring lasers with the 895 etalon assembly installed to reduce the linewidth of the beam. One uses the Coumarin 480 dye and the other one uses a DCM special dye. To monitor the quality of the dyes, two spectrometers are used. A Varian Cary Eclipse measures the fluorescence 
spectra and a Perkin Elmer Lambda 800 UV/vis measures the absorption spectra. Typical fresh absorption and fluorescence spectra of both dyes used are plotted in Figure 4.5 .

A krypton ion Sabre laser from Coherent Innova is used to pump the Coumarin 480 dye laser. It is a $2 \mathrm{~W}$ ultraviolet laser used in a multi-line configuration. The DCM Special dye is pumped by a Verdi 8 solid state laser, producing a maximum of $8 \mathrm{~W}$ at $532 \mathrm{~nm}$. Both ring lasers give a typical energy output of 100 to $200 \mathrm{~mW}$. A Newport 1830-C digital power meter monitors the laser power. The beam quality is measured with a Newport LBP-2-USB beam profiler which shows a digital image of the geometry and intensity of the laser beam. To monitor the wavelength of the beams, a flat transparent glass disk is used to sample a portion of the beam. Then, a collector focuses the beam on an optical fibre which carries the light to an EXFO wavemeter WA-1500 spectrometer which sends the spectrum information to an EXFO WA-650 Fourier analyzer. It finally sends the analyzed spectrum to a computer. A typical snapshot of laser spectra with and without the etalon assembly using the Fourier analyzer can be seen in Figure 4.6.

\subsection{Electrical Discharge Ion Source}

In the first part of the experiment, an arc was used to evaporate the barium and to ionize it. The arc was produced between two copper electrodes by an ECO-10 electronic fluorescent dimming ballast from Lutron. The source was pure barium granules from Sigma-Aldrich and one was placed inside a cavity in the lower electrode as seen in Figure 4.7. The distance between the electrodes was about $10 \mathrm{~mm}$ and the potential difference between the two electrodes was less than $200 \mathrm{~V}$. Pressures of a few mbar was the only regime where arcing was possible due to the limitations of the ballast. Figure 4.7 also shows electric field shaping rings that could drift away from the arc before performing the LIF. Due to the technical difficulty of applying a uniform drift voltage, LIF was performed at the location 


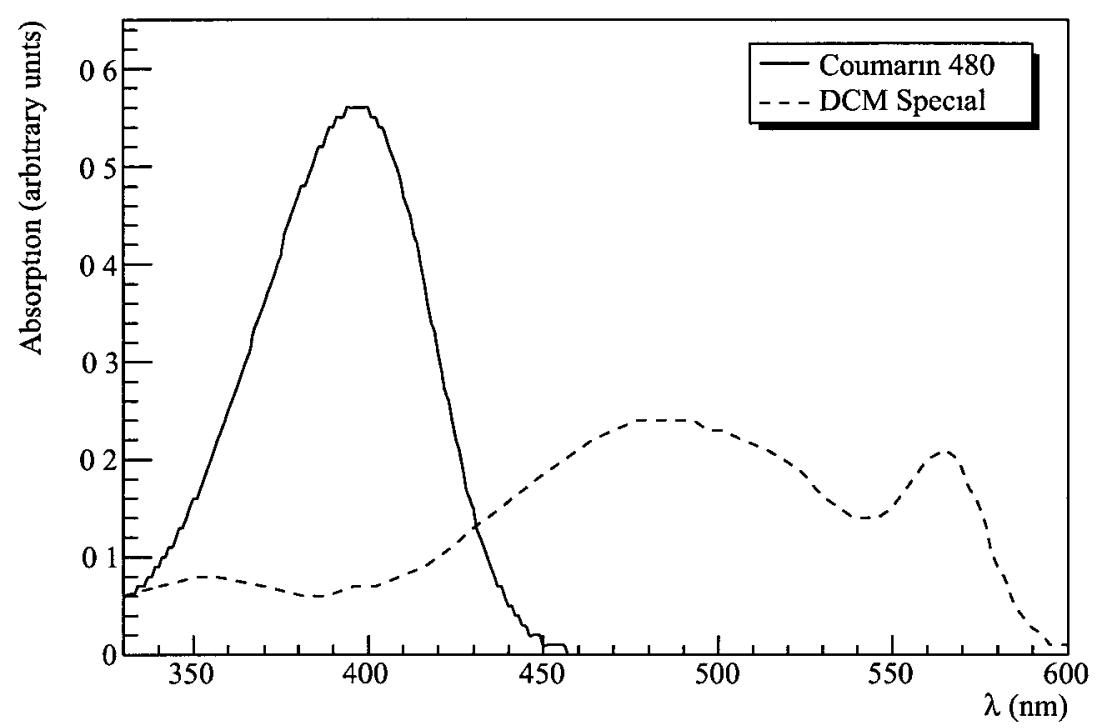

(a)

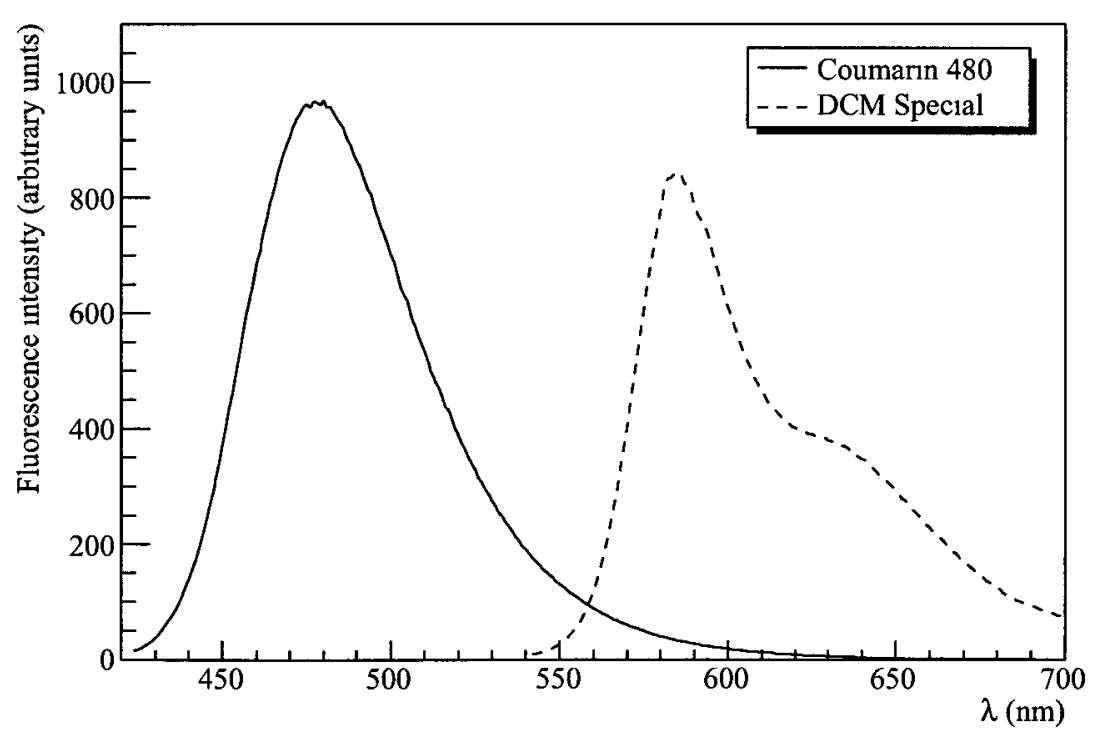

(b)

Figure 4.5: (a) Absorption and (b) fluorescence spectra of the Coumarin 480 and the DCM Special dyes in arbitrary units as a function of the wavelength. 


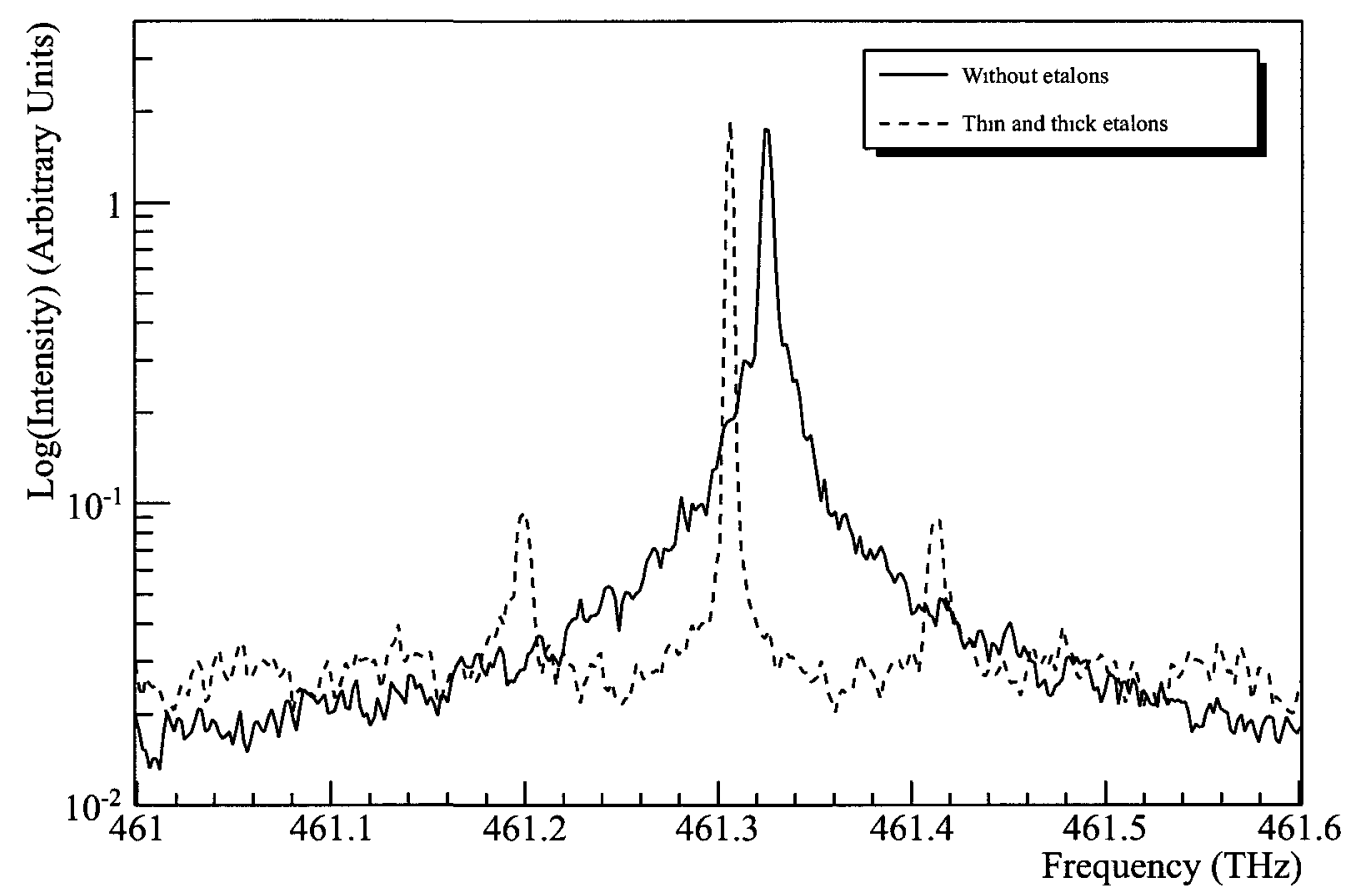

Figure 4.6: Typical dye laser spectra with and without the etalon assembly. 


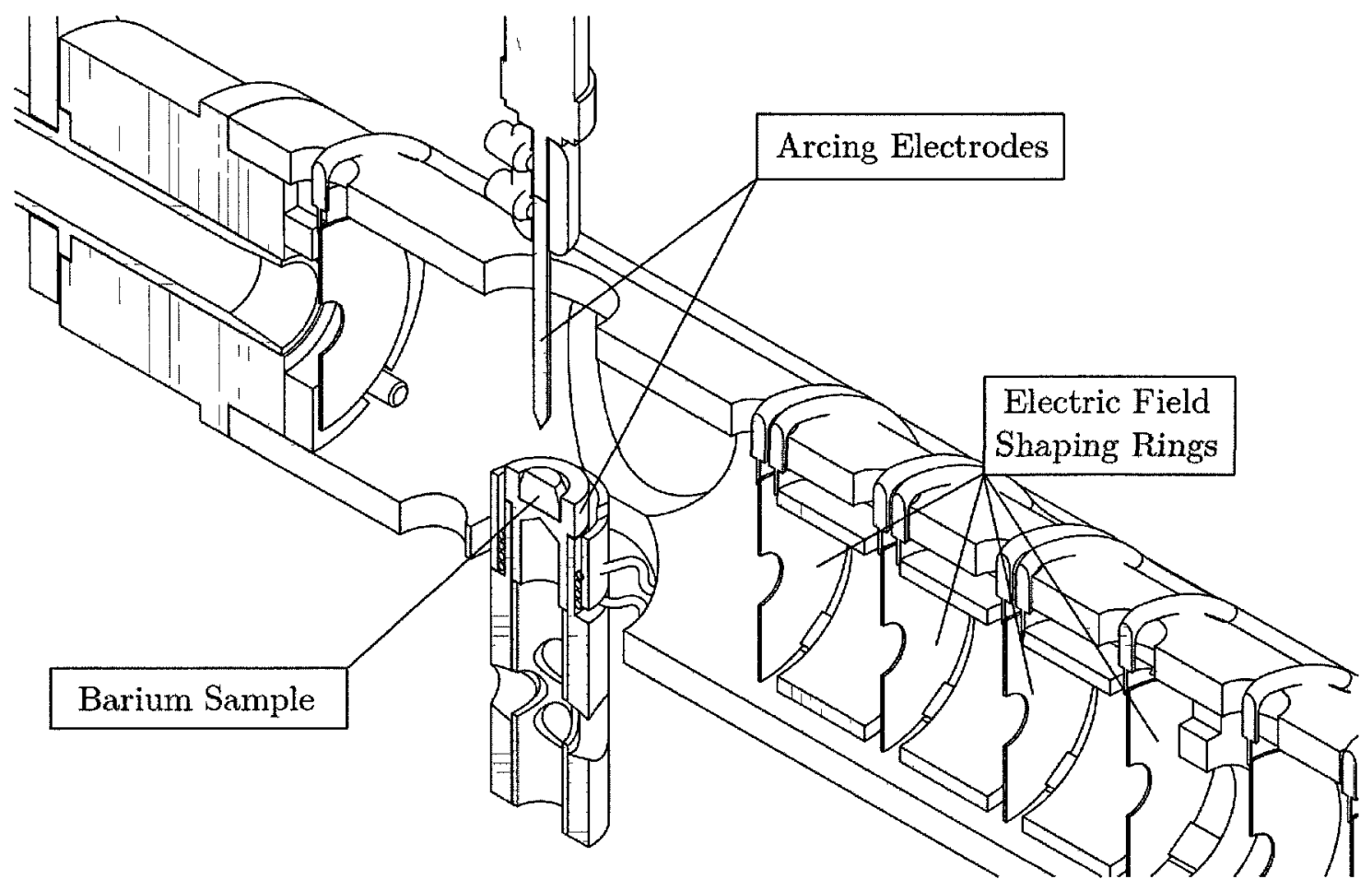

Figure 4.7: Schematic of the arcing chamber used in the study of barium fluorescence from electrical discharges.

of the electrical discharge.

The assembly was placed in a vacuum chamber made of stainless steel components. The chamber was built from a 6 -way cube accommodating $23 / 4$ " ConFlat flanges, a 6-way cross and various nipples and flanges. The vacuum level was monitored by a $943 \mathrm{MKS}$ cold cathode ionization gauge while the gas pressure was measured with a MKS Baratron capacitance manometer. A gas system was built from stainless steal tubing. It handled two gas bottles, both gas paths going through Agilent moisture traps. One of them passes through a Chromatographic Specialities oxygen trap followed by an Oxyclear oxygen trap. The pumping was done by a Varian Task-V70LP pump which consists of a turbomolecular pump backed up by a scroll pump in the same package. 


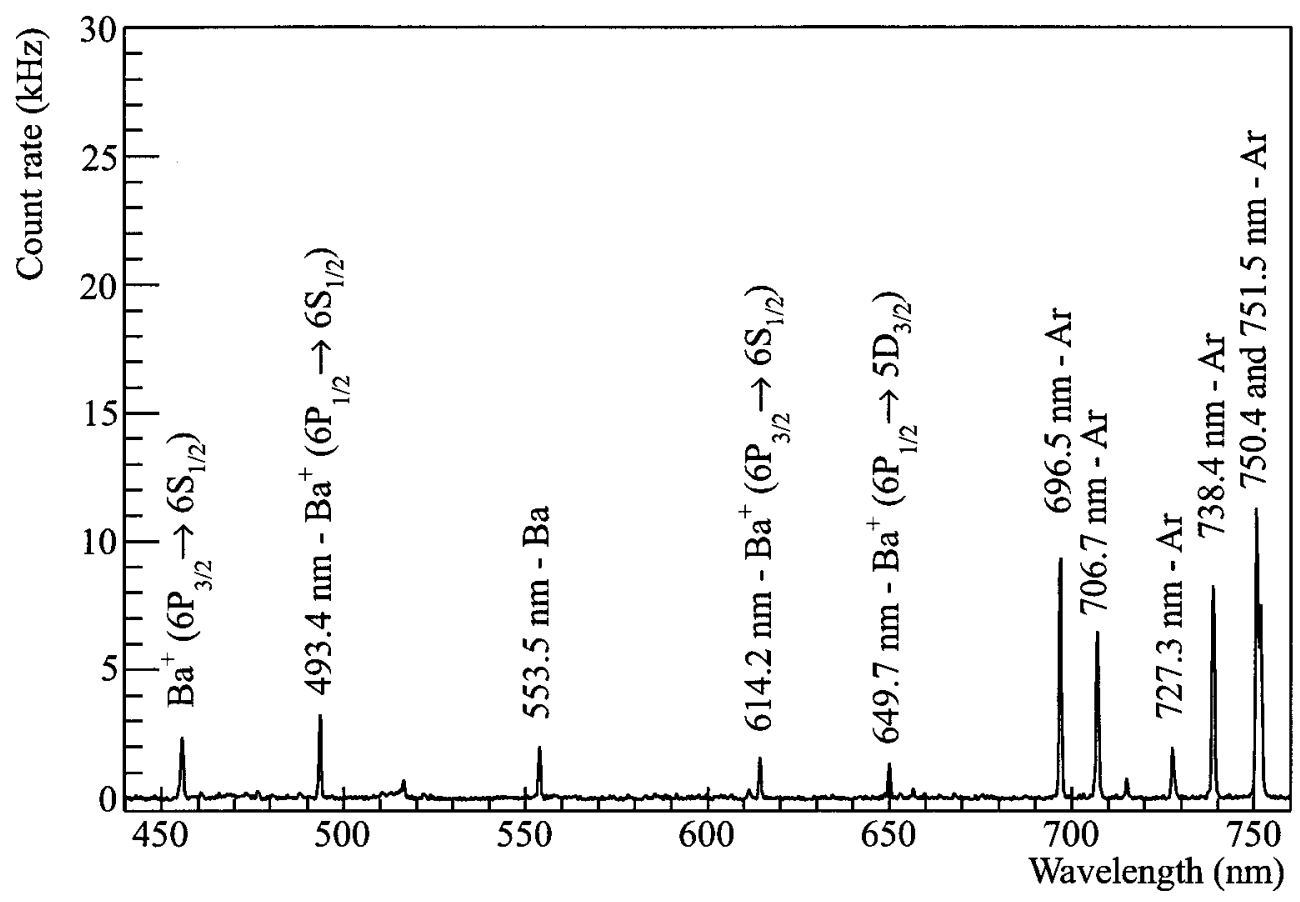

Figure 4.8: Emission spectrum of the arc using barium metal as a source.

\subsubsection{Barium Fluorescence Data from Electric Discharges}

Barium atoms and ions are produced in the ground and excited states from the heat of the arc and the collisions against high energy electrons. In order to sustain a well defined arc, argon gas was added to the chamber with a pressure of about 30 mbar. The light from the arc was analyzed with an Ocean Optics HR4000CG-UV-NIR spectrometer and a typical emission spectrum is depicted in Figure 4.8 .

Besides barium ion lines, neutral barium and argon lines were also visible. To reduce the noise level, the average of ten spectra was recorded in the same condition. The background signal was removed by fitting a fifth order polynomial on $70 \%$ of the spectra, away from visible emission lines and then subtracting it. The 
dataset is composed of 101 averaged spectra, each having slightly different operating conditions (pressure, voltage, fluorescence laser intensity and wavelength).

Pictures of the LIF is shown on Figure 4.9. The two electrodes and the vertical arc in between are visible. The horizontal fluorescence laser beam is also visible. In Figure 4.9a, the fluorescence laser is tuned to $493 \mathrm{~nm}$ and fluorescence is visible where the laser beam interacts with the $\mathrm{Ba}^{+}$cloud. Fine dust generated by the arc scatters the blue laser and is visible at the far left and right of the picture. In Figure $4.9 \mathrm{~b}$, the $650 \mathrm{~nm}$ laser is used to excite the $\mathrm{Ba}^{+}$ions produced in the $D_{3 / 2}$ state by the arc. Then, the decay of the $P_{1 / 2}$ state occurs emitting predominantly blue light. Note how the $650 \mathrm{~nm}$ light is shifted to $493 \mathrm{~nm}$ light where $\mathrm{Ba}^{+}$is produced.

\section{Branching Fraction Measurement}

The first measurement possible from the processed spectra is the branching fraction (BF) of the decay of the $P_{1 / 2}$ state into the $\mathrm{T} S_{1 / 2}$.his fraction is calculated from the intensity of the $493 \mathrm{~nm}$ line compared to the $650 \mathrm{~nm}$ line. Light collected comprises that generated by the arc, the scattered laser light as well as the laser induced fluorescence. The branching fraction distribution for the 101 spectra recorded is shown in Figure 4.10.

The BF found was $0.74 \pm 0.04$ which agrees within the uncertainty of the measurement with the value calculated by Gallagher [48] of 0.735 . The large standard deviation of the measurement could be due to a dependence of the BF on the pressure. The data recorded did not show any significant pressure dependence but the pressure reading was uncertain. The MKS Baratron manometer was not available at the time, so the pressure was measured with a Bourdon gauge which had lower accuracy. Another possible explanation for the broad distribution is the presence of some $493 \mathrm{~nm}$ and $650 \mathrm{~nm}$ scattered laser light in spectra. Great care was taken in selecting configurations for which the lasers had very low intensity, much lower than the actual fluorescence light, but it is a possibility that fluctuation 


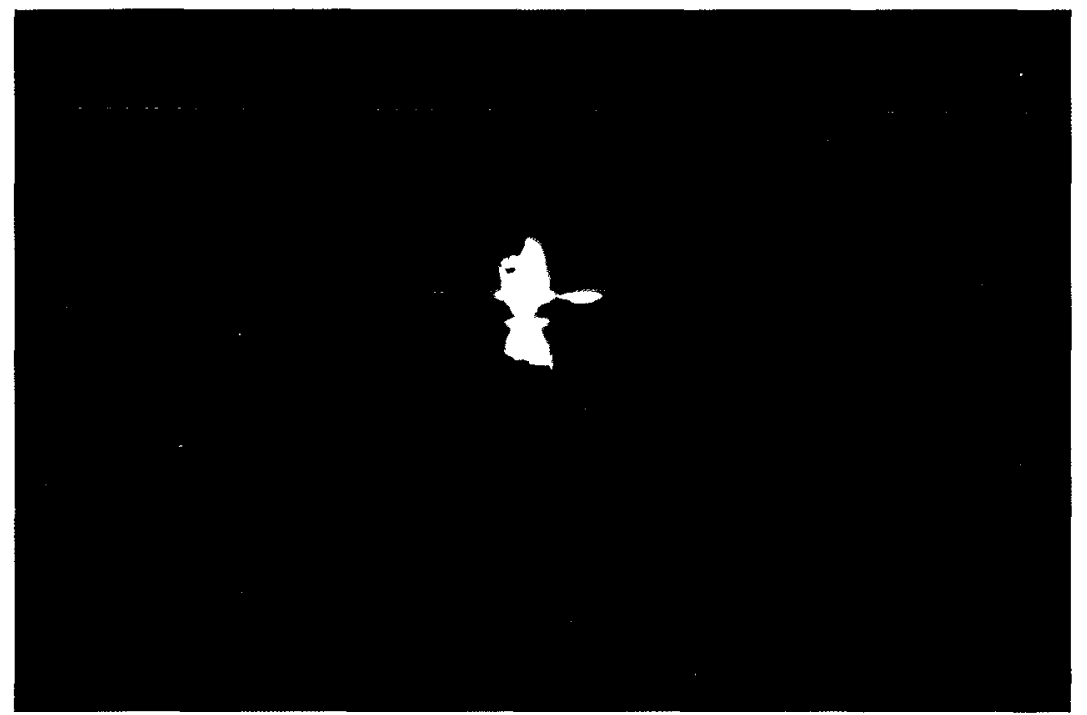

(a)

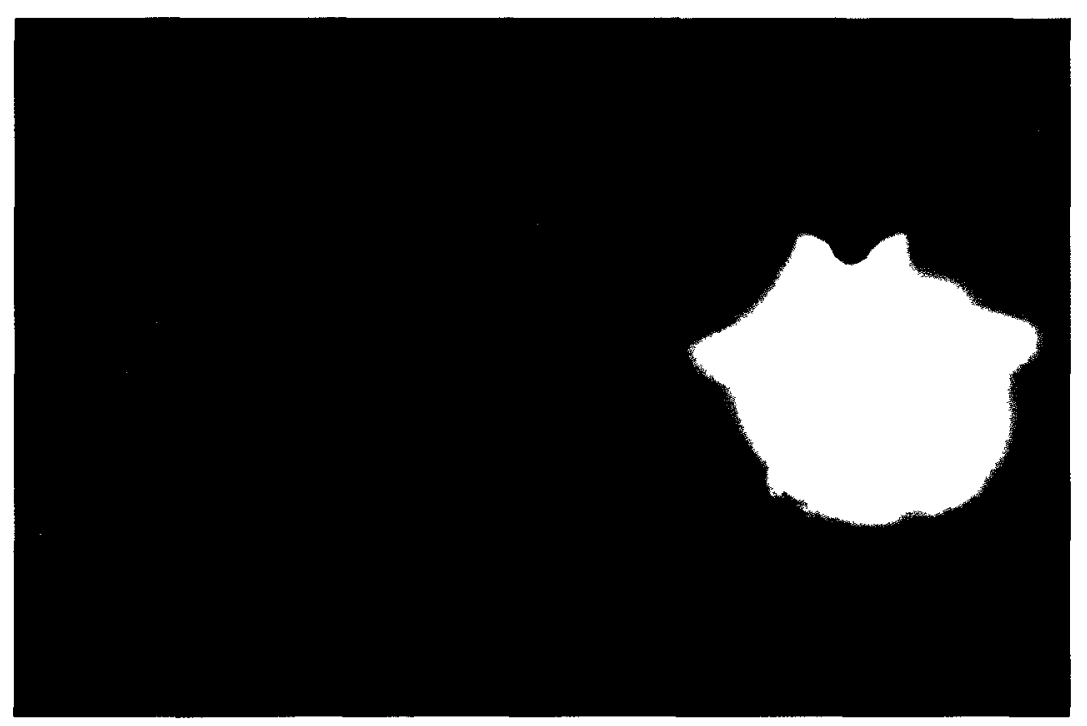

(b)

Figure 4.9: Photographs of the LIF of $\mathrm{Ba}^{+}$ions generated by the electric discharge ion source. (a) shows the excitation of the $S_{1 / 2}$ state to the $P_{1 / 2}$ state by the blue laser and its decay. The horizontal line near the electrodes is actual fluorescence. (b) shows the excitation of the metastable $D_{3 / 2}$ state to the $P_{1 / 2}$ state by the red laser and then its fluorescence. 


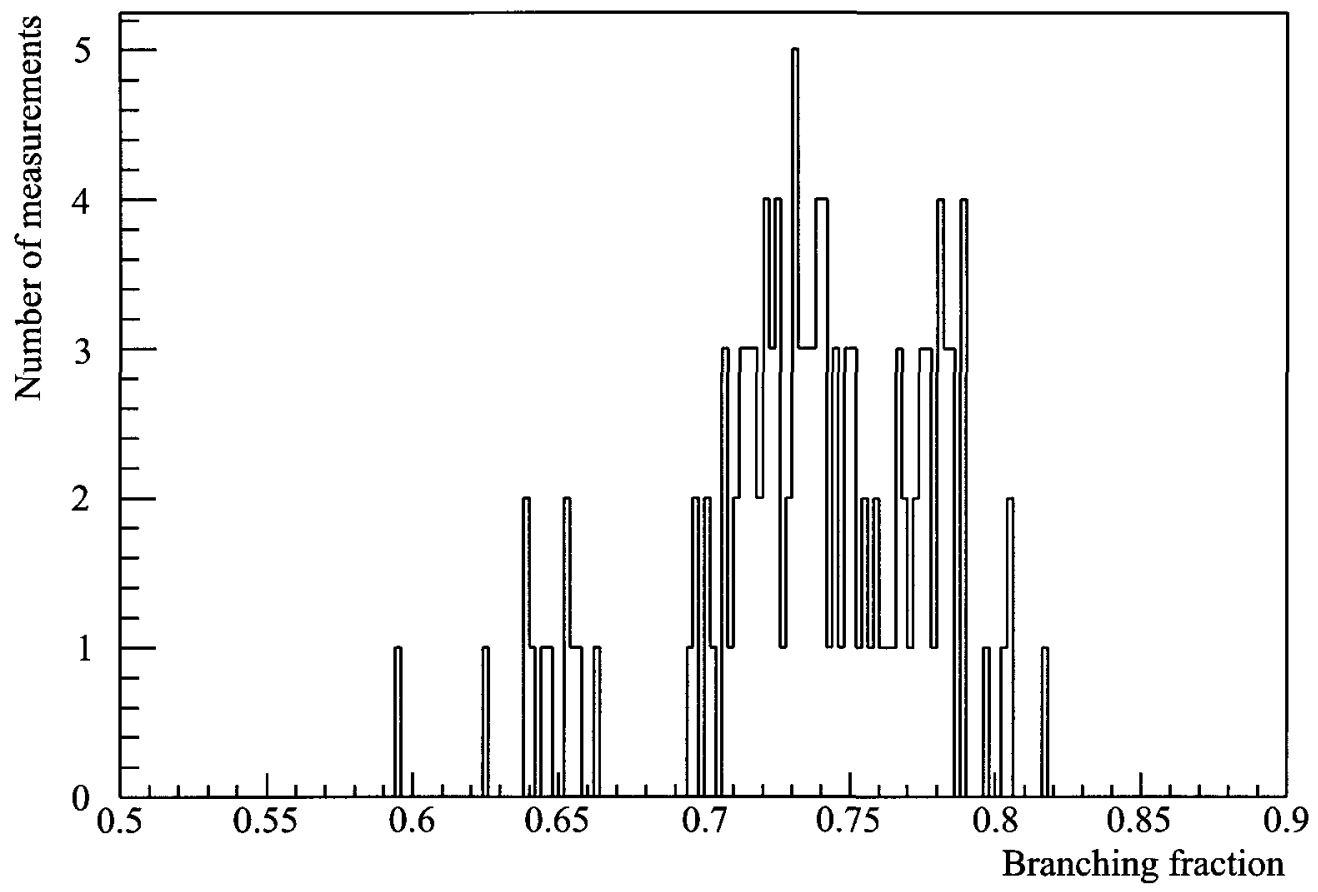

Figure 4.10: Branching fraction of the $P_{1 / 2}$ state decay to the $S_{1 / 2}$ and $D_{3 / 2}$ states. 
in the laser light intensity occurred and shifted the BF up or down. Nevertheless, this measurement confirmed the viability of the chamber.

\section{Linewidth and Position Measurements}

The second measurement related to $\mathrm{Ba}^{+}$spectroscopy is the line position and width of the $S_{1 / 2}-P_{1 / 2}$ transition. This was measured by slowly scanning the wavelength of the $493 \mathrm{~nm}$ laser and by looking at the fluorescence intensity of the $650 \mathrm{~nm}$ line. Since this intensity is proportional to the amount of $\mathrm{Ba}^{+}$produced and the source was very unstable, the $650 \mathrm{~nm}$ line intensity had to be normalized with the two $\mathrm{Ba}^{+}$lines from the decay of the $P_{3 / 2}$ state, at $455 \mathrm{~nm}$ and $614 \mathrm{~nm}$, which are present in the spectra and are a good indicator of the amount of barium produced. The normalization ratio used is:

$$
\text { Ratio }=\frac{\mathrm{I}(650)}{\mathrm{I}(455)+\mathrm{I}(614)}
$$

Then, the constant background was subtracted and the line was fitted with the empirical approximation of a Voigt function from Whiting [60]. That way, the Gaussian and the Lorentzian components of the line have been extracted. The data points and the fitted line are shown in Figure 4.11.

The fitted position was $493.5470 \mathrm{~nm}$ where the expected position in vacuum was $493.5454 \mathrm{~nm}$. The $+0.0016 \mathrm{~nm}$, or $-2094 \mathrm{MHz}$ shift, shows the large attractive force between the $\mathrm{Ba}^{+}$and the surrounding $\mathrm{Ar}$ atoms. Knowing that the data was taken at $30 \mathrm{mbar}$, this translates into a $-132 \mathrm{MHz} /$ Torr shift. Error bars have been

omitted since the estimation of their value is difficult. Instead, the scattering of the individual data points displays the size of the total uncertainty.

The measured FWHM of the line was $1.27 \pm 0.12 \times 10^{-3} \mathrm{~nm}$, where the uncertainty is only due to the fit and not the instruments. Table 4.1 enumerates the broadening sources.

Unfortunately, the dominant source for the width and its uncertainty is the 


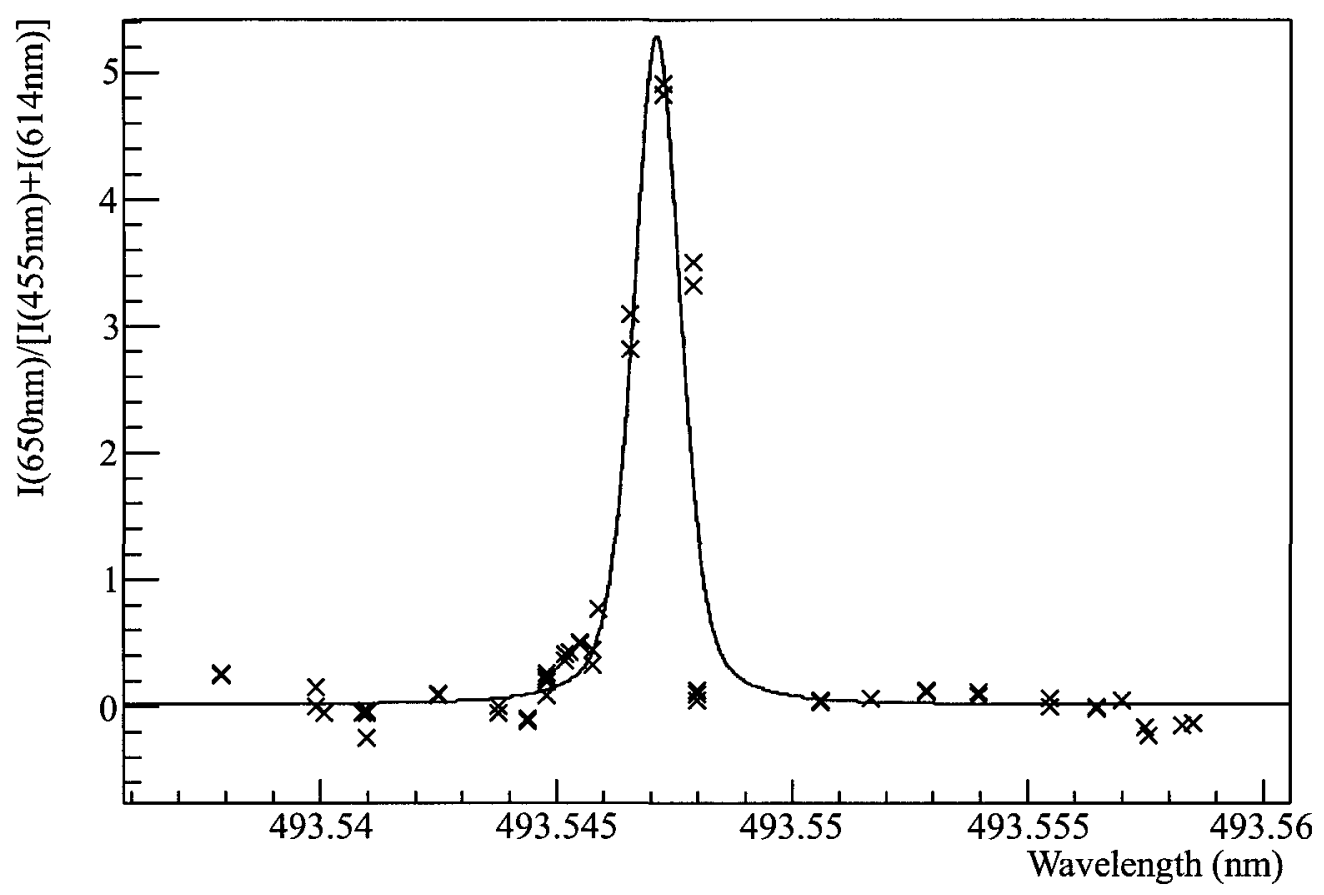

Figure 4.11: Line profile of the $S_{1 / 2}-P_{1 / 2}$ transition at 30 mbar of argon.

\begin{tabular}{|c|c|}
\hline Source & Linewidth $\left(\times 10^{-3} \mathrm{~nm}\right)$ \\
\hline \hline Natural & $0.05 \pm 0.02$ \\
\hline Doppler & $0.68 \pm 0.46$ \\
\hline Instrumental & $0.15 \pm 0.03$ \\
\hline \hline Total & $0.70 \pm 0.47$ \\
\hline Measured value & $1.27 \pm 0.12$ \\
\hline
\end{tabular}

Table 4.1: List of known broadening causes of the $P_{1 / 2}-S_{1 / 2}$ transition. Collision broadening is assumed to be the difference between the measured value and the total expected value. 
temperature of the source. A typical arc temperature is between 3000 and $8000 \mathrm{~K}$, but the temperature where the fluorescence is obtained can be as low as the room temperature of $297 \mathrm{~K}$.

Using the total estimated value for the linewidth, $0.57 \pm 0.48 \times 10^{-3} \mathrm{~nm}$ is unaccounted for and can be assigned to collisional broadening. This can be converted to $702 \pm 591 \mathrm{MHz}$, or $23 \pm 20 \mathrm{MHz} /$ Torr, very similar to the measured value by Zokai et al. [59] of $19 \mathrm{MHz} /$ Torr, despite the large uncertainty.

The electrical discharge ion source was too unstable and difficult to control for the barium tagging investigation. The source was also incapable of generating ions at higher pressures. Therefore, another option to create $\mathrm{Ba}$ ions was considered.

\subsection{Laser Ablation Ion Source}

Ions can be produced by a high energy density laser beam focused on a solid target. When the fluence is large enough, the electromagnetic field excites the electrons of the conduction band of the target and phonons are produced within the first $1 \mathrm{ps}$. The heat ejects material from the surface in neutral and ionized states in a semitransparent plume. Then, photons of the laser beam heat up the plasma by giving energy to electrons via the inverse bremsstrahlung effect. Accelerated electrons can collide with neutral atoms and ionize even more of the plume making the cloud progressively opaque. Finally, the plume expands asymptotically in vacuum or collides with the gas and is thermalizes within several $\mu \mathrm{s}$. When the laser beam stops, the target is still hot and can generate ions by desorption. These ions tend to be lower in number than the ones produced by the collision of the electrons and the vapourized material.

Such ablation begins at the energy densities required for the vapourization of the material. Above this threshold, the amount of material extracted increases more or less linearly as a function of the energy density of the laser beam and the duration of the pulse. The time at which vapourization occurs $\left(t_{v}\right)$ can be 
calculated with Equation 4.30, taken from Andreev et al. [61]:

$$
t_{v}=\frac{\chi t_{p}^{2} \rho C_{p} T^{2}}{[(1-R) F]^{2}}
$$

where $\chi$ is the thermal conductivity, $t_{p}$ is the pulse duration, $\rho$ is the target density, $C_{p}$ is the specific heat, $T$ is the temperature reached, $R$ is the reflectivity and $F$ is the laser fluence. Vapourization occurs quickly for low reflectivity material with a low thermal conductivity, low density and low specific heat. The fluence at threshold $\left(F_{t h}\right)$ can be calculated using the temperature of the boiling point of the material and by setting the vapourization time to be the pulse duration $\left(t_{v}=t_{p}\right)$ giving:

$$
F_{t h}=\frac{\sqrt{\chi t_{p} \rho C_{p} T_{v}^{2}}}{(1-R)}
$$

For copper, the threshold is around $2.2 \mathrm{~J} / \mathrm{cm}^{2}$. For barium, since the vapourization temperature, the heat capacity and the thermal conductivity are much lower, the threshold is around $0.15 \mathrm{~J} / \mathrm{cm}^{2}$.

The ablation laser used is a Explorer Scientific pulsed UV laser producing $349 \mathrm{~nm}$ light. It can generate a maximum energy of $120 \mu \mathrm{J}$ per pulse within $5 \mathrm{~ns}$. Therefore, the beam has to be focused to roughly $300 \mu \mathrm{m}$ or smaller to produce $\mathrm{Ba}^{+}$. The lens used is expected to focus to a diameter of 5 to $10 \mu \mathrm{m}$, creating a pulse beyond the threshold density.

The plume, when created in vacuum, is similar to a nozzle source. Its angular profile follows a $\cos \theta$ distribution if the density is low and as the density increases, the plume becomes more forward peaked and tends toward a $\cos ^{4} \theta$ distribution. According to Amoruso et al. [62], the energy distribution follows a Maxwell-Boltzmann distribution with a superimposed hydrodynamic flow velocity. The number of ions per unit of energy can be described as: 


$$
N(E) d E=C \sqrt{\frac{m v_{x}^{2}}{2}} \exp \left[-\frac{m\left(v_{x}-v_{c m}\right)^{2}}{2 k_{B} T}\right] d E,
$$

where $C$ is a dimensional constant, $v_{x}$ the velocity of the ion perpendicular to the surface, $v_{c m}$ is the center of mass velocity of the cloud, $m$ is the mass of the ion, $k_{B}$ the Boltzmann constant and $T$ the temperature of the surface of the target.

At $3 \mathrm{~J} / \mathrm{cm}^{2}$ over a surface of $7 \times 10^{-4} \mathrm{~cm}^{2}$ and using a copper target, Kools et al. [63] estimated that they produced $8 \times 10^{6}$ ions per laser pulse while the total amount of material ablated was about $5 \times 10^{14}$ atoms per pulse. Therefore, the ionized fraction of $\mathrm{Cu}$ at macroscopic distances from the target is of the order of $10^{-8}$. This number is highly dependant on the laser fluence. They also report a considerable fraction of Rydberg atoms. Therefore, generating an electric field in the vicinity of the plume increases the ion yield.

In metals, the laser light is absorbed within the first $10 \mathrm{~nm}$. In fact, the amount of mass a laser ablates ( $\dot{m}$ in $\mathrm{g} \mathrm{cm}^{-2} \mathrm{~s}$ ) is derived by Phipps et al. [64]:

$$
\dot{m}=2.66 \times 10^{-6} A^{-1 / 4}\left(\frac{A}{2\left[Z^{2}(Z+1)\right]^{1 / 3}}\right)^{9 / 8}\left(\frac{I}{\lambda \sqrt{\tau}}\right)^{1 / 2}
$$

where $A$ is the mass number, $Z$ is the atomic number, $I$ is the intensity of the laser beam in $\mathrm{W} / \mathrm{cm}^{2}, \lambda$ is the wavelength in $\mathrm{cm}$ and $\tau$ is the duration of the pulse in s. For barium with $A=137$ and $Z=56$ and for the laser used, $\tau=5 \times 10^{-9} \mathrm{~s}$, $\lambda=3.49 \times 10^{-5} \mathrm{~cm}$, Equation 4.33 becomes:

$$
\dot{m}_{\mathrm{Ba}}=170 \sqrt{I} \text {. }
$$

Therefore, at an energy density of $2 \mathrm{~J} / \mathrm{cm}^{2}$ and a spot size of $3.1 \times 10^{-6} \mathrm{~cm}^{2}$, the mass ablated should be around $3 \times 10^{-12} \mathrm{~g} /$ pulse, or $2 \times 10^{12} \mathrm{Ba}$ atoms per pulse. Using the charge induced on the cathode, the typical number of ions detected was between $10^{4}$ and $10^{7}$ ions. Therefore, the ionization fraction is roughly $10^{-8}$ to $10^{-5}$, similar to other experiments. 


\subsubsection{Laser Beam Size and Intensity}

To measure the size of the beam at the focus point, a razor blade was swept across the beam and the intensity was measured as a function of the position. Then, the width of the Gaussian function was found by fitting an error function on the intensity curve:

$$
\operatorname{erf}(x)=A \int_{0}^{x} e^{t^{2}} d t
$$

The size of the beam was found to be typically $10 \mu \mathrm{m}$ which is much smaller than the size of the damage seen through a microscope (Figure 4.12). This can be explained by vibrations of the laser or of the target, some backlash in the micrometre screw and the laser beam not being focused perfectly but still creating some damage on the target. It is important to note that $10 \mu \mathrm{m}$ is an upper limit. Even at the lowest intensity, when the laser beam was perfectly focused on the razor blade, it was slowly damaging the edge of the blade. This had the effect of increasing the measured size of the beam.

\subsubsection{Ablation Rate Decay}

A problem of using a laser ablation ion source is the quick decay in the signal. This has been observed by many scientists. If the laser is kept focused on the same point for a long period of time, the intensity of the signal decreases. A typical decay curve using pure barium is plotted in Figure 4.13.

The higher the power density of the laser, the faster the signal decays. Presence of gas also increased the rate of the decay. The production rate can be brought back by moving the location of the focus point by a small amount. The cause of this decay is not clear. It is possible that the slow oxidation of the surface in air makes the first few atomic layers easier to ablate or that the heat of the laser transforms the surface of the target so that it is more difficult to ablate. 


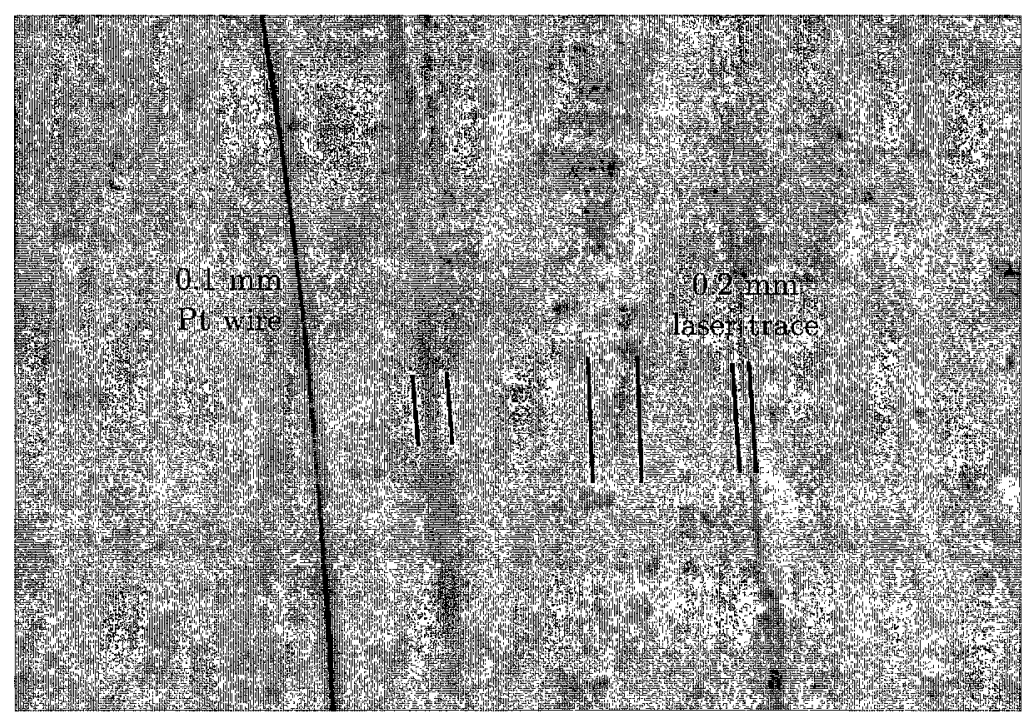

Figure 4.12: A view of a $0.1 \mathrm{~mm} \mathrm{Pt}$ wire, on the left, juxtaposed to three different damage traces left by the ionization laser on the target, on the right.

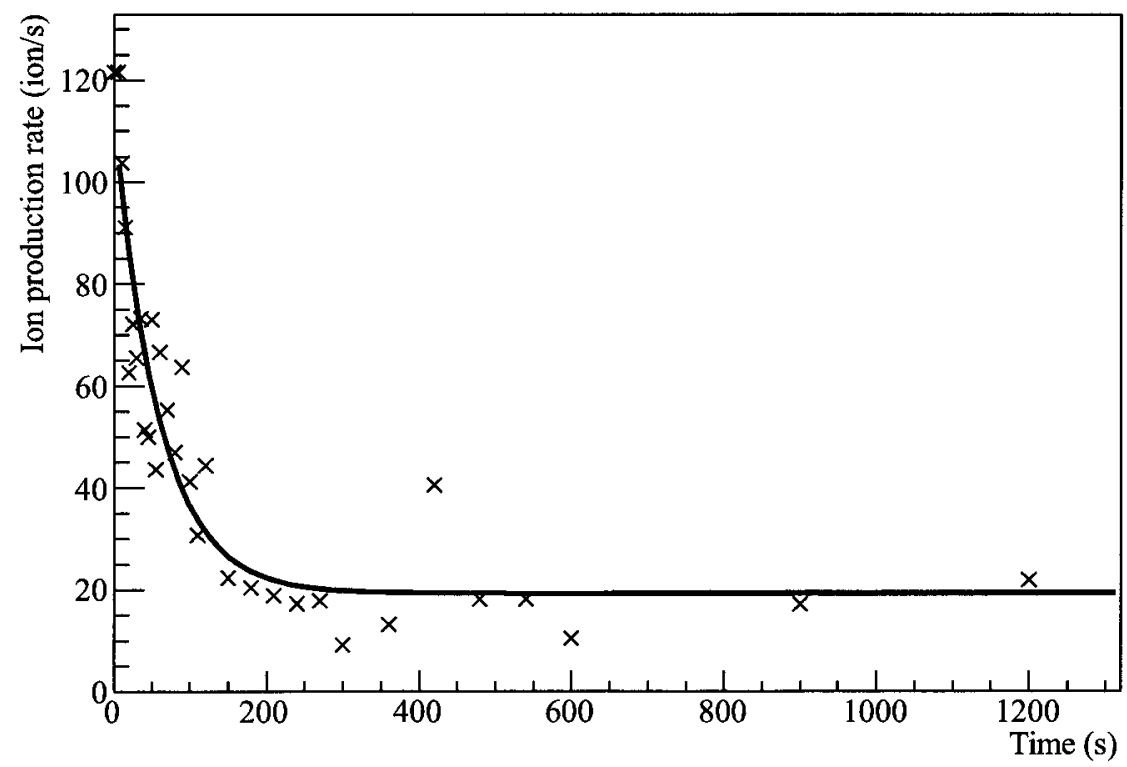

Figure 4.13: Typical decay of the ion signal with time. 


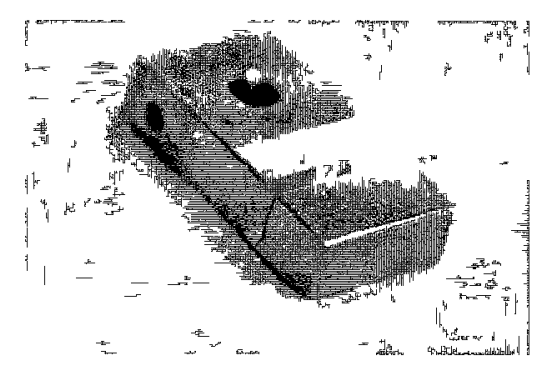

Figure 4.14: Copper mount for the barium target. A barium shard goes in the groove. The larger hole in the top of the target is used to affix the mount to the manipulator and the smaller hole on the side of the target is used to establish an electrical connection. The mount is $1 \mathrm{~cm}$ wide.

\subsection{Time of Flight of Ions from Laser Ablation}

The target used was a pure barium metal needle placed at an angle on a flat copper mount. The mount was placed at a positive potential, typically $200 \mathrm{~V}$, and its surface was perpendicular to the drift direction. The focused laser comes parallel to the surface of the mount and perpendicular to the drift direction. A small groove was cut in the mount at an angle, to offer a surface for the laser to hit. Barium metal was then cut into a small shard and placed in the groove. That way, the laser could hit the barium sample and the copper mount could create its electric drift field in the right direction. Figure 4.14 shows a picture of the bare copper mount, with the barium target absent from the groove. Focus of the laser beam was obtained by moving the target with a 3-axis manipulator at the internal lens focus point. The lens used was a Newport reversed Cassegrain microscope objective.

After leaving the target, ions are focused by an Einzel lens assembly and $200 \mathrm{~mm}$ further down the drift direction, go through a $3 \mathrm{~mm}$ aperture. They are deflected by a charged plate, onto a channel electron multiplier (CEM). The CEM is from Burle, model number 4502 which can detect ions. The signal is then amplified by a Amp-Tek A121 preamplifier-discriminator. An Ortec Multi-Channel 
Scaler (MCS) measures the time spectrum.

The design of the chamber is shown in Figure 4.15. The apparatus was modelled in the SIMION software. This software simulate the interaction of charged particles with the electric field generated by elements set at different potentials. It also allows interactions between a charged particle and the surrounding gas, or even in between multiple charged particles. Examples of ion paths in the apparatus are shown in Figure 4.16.

\subsection{Time of Flight Theory}

The time of flight (ToF) chamber was built to determine the composition of the ejected material. The arrival time of ions is proportional to the square root of the mass to charge ratio. The working principle is simple, ions are produced in an electric field and their potential energy $q U$ is converted into kinetic energy $K$ :

$$
\begin{gathered}
q U=K \\
q U=1 / 2 m(d / t)^{2}, \\
\mathrm{ToF}=\sqrt{\mathrm{md}^{2} / 2 \mathrm{qU}} .
\end{gathered}
$$

By defining $A=\sqrt{d^{2} / 2}$ and setting $U$ as the voltage of the target $V$, the ToF becomes as simple as:

$$
\mathrm{ToF}=\mathrm{A} \sqrt{\mathrm{m} / \mathrm{qV}}
$$




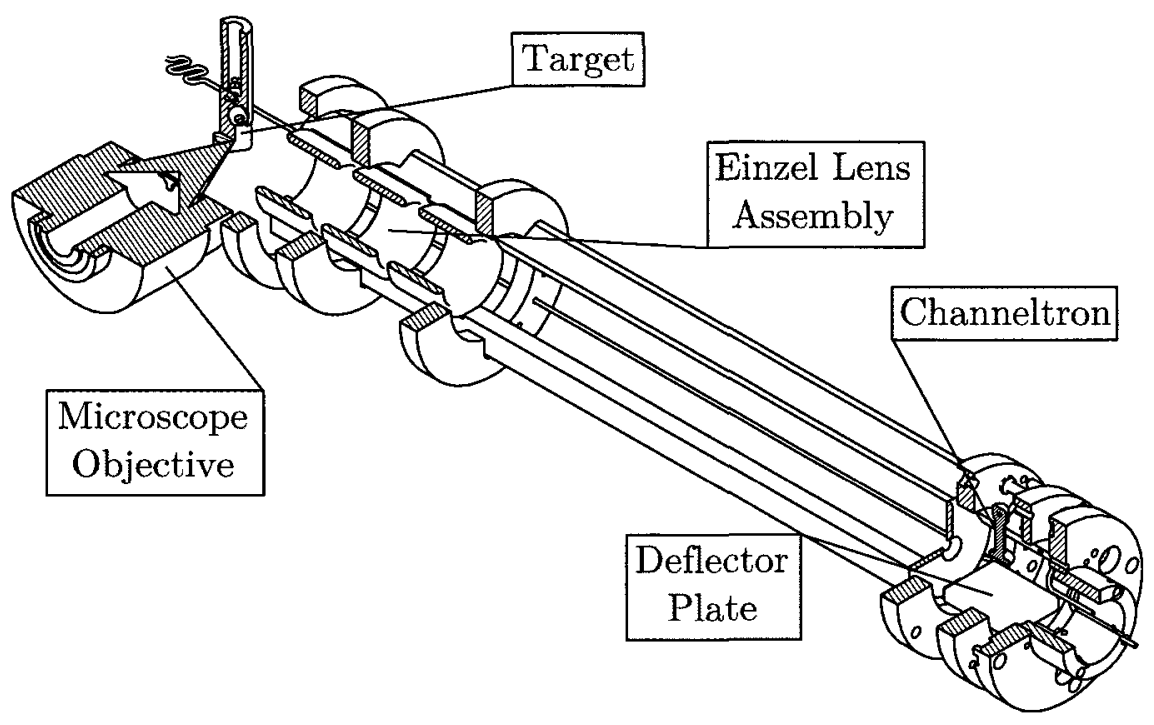

Figure 4.15: ToF Chamber schematic.

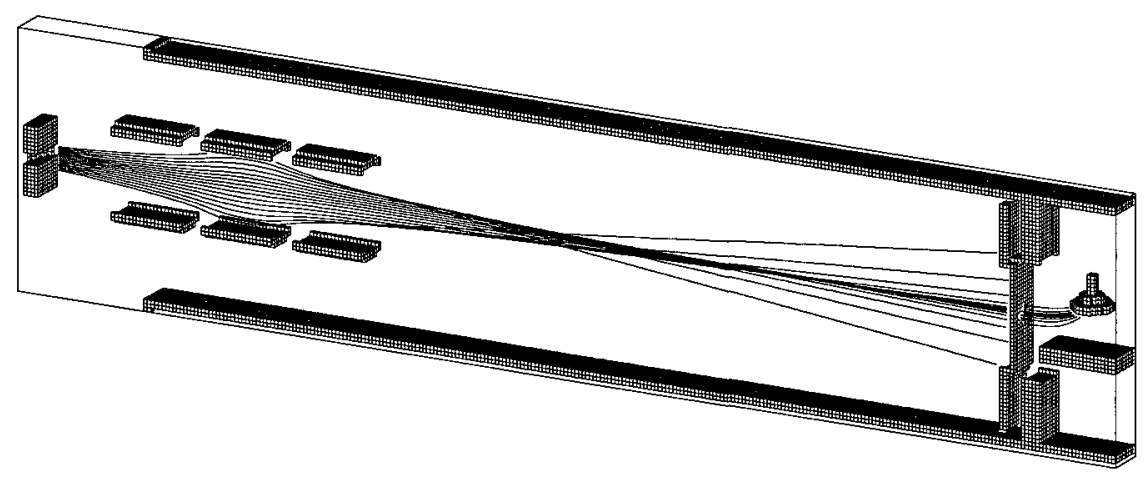

Figure 4.16: Simulation of ion paths through a cut of the ToF chamber using SIMION. 


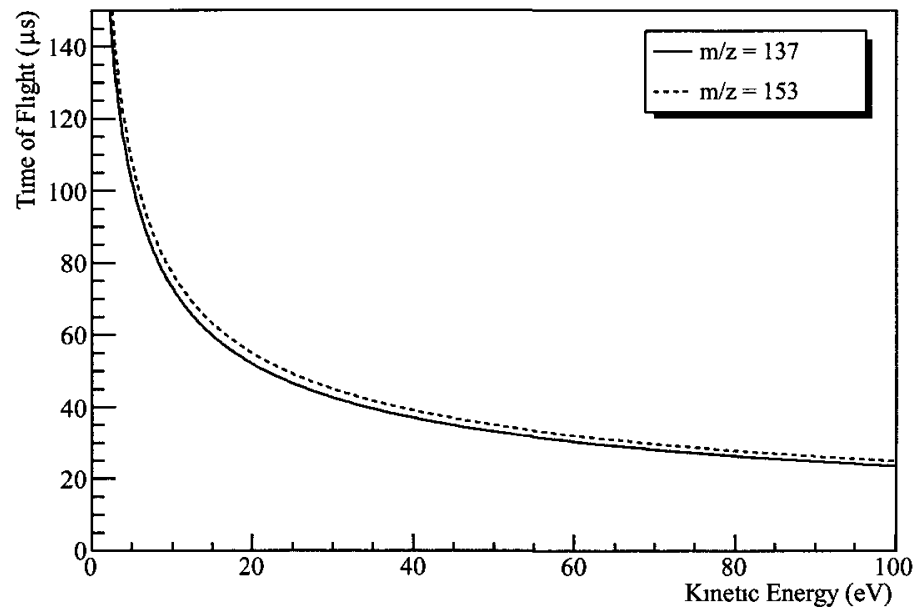

Figure 4.17: Simulated time of flight of $\mathrm{Ba}^{+}(\mathrm{m} / \mathrm{z}=137)$ and $\mathrm{BaO}^{+}(\mathrm{m} / \mathrm{z}=153)$ in a model of the chamber as a function of their initial kinetic energy and no drift voltage.

\subsubsection{Ion Energy Spectrum}

By applying only a voltage on the CEM to collect ions and keeping other elements grounded, it is possible to estimate the kinetic energy of ions leaving the target. Their ToF can be calculated with Equation 4.39, by replacing the potential energy by their initial kinetic energy $\left(E_{k}\right)$, the mass of the particle $(m)$ and the distance travelled $(D)$.

$$
\mathrm{ToF}=\mathrm{A} \sqrt{\frac{\mathrm{m}}{\mathrm{E}_{\mathrm{k}}}}
$$

The constant $A$ can be found using SIMION to simulate ions in a model of the chamber. Figure 4.17 shows the ToF calculated for two different ion masses.

The fitted curve gives:

$$
\mathrm{ToF}=19.53 \sqrt{\frac{\mathrm{m}}{\mathrm{E}_{\mathrm{k}}}}
$$


with the mass in $\mathrm{Da}$, the energy in $\mathrm{eV}$ and the ToF in $\mu \mathrm{s}$. The energy distribution of ions should follow a Maxwellian distribution described by:

$$
N d E=2 \sqrt{\frac{E}{\pi\left(k_{B} T\right)^{3}}} \exp \left[\frac{-E}{k_{B} T}\right] d E
$$

Figure 4.18 shows the ToF spectrum of barium in the chamber without applying any drift voltage. The large peak is composed of two main resolvable peaks. Unfortunately, the Maxwellian distribution described by Equation 4.42 does not fit the peaks. A Maxwellian distribution has a long tail at large values of time of flight. The absence of such tails can be explained by a forward velocity caused by a stray electric field or some cloud repulsion effect, boosting ions giving an energy distribution more Gaussian than Maxwellian. Another explanation could be the inefficiency of detecting low energy ions. Tails would be composed of the slowest particles, which would be more easily diverted by stray electric fields or residual gas. In any case, the bulk of the distribution is the important one. Gaussian distributions can be fitted without losing the ability to calculate the mean kinetic energy from the ToF.

A fit of the two main peaks gives a mean of $61.2 \mu \mathrm{s}$ and a sigma of $8.8 \mu \mathrm{s}$ for the first peak and a mean of $69.3 \mu \mathrm{s}$ with a sigma of $7.2 \mu \mathrm{s}$ for the second one. As Section 4.6.3 will show, it is reasonable to assign the first peak to $\mathrm{Ba}^{+}$and the second one to $\mathrm{BaO}^{+}$. Other components could be present, although for a first order measurement, these are good assumptions. The initial kinetic energy of ions are therefore $14.0_{-3.3}^{+5.1} \mathrm{eV}$ for $\mathrm{Ba}^{+}$and $12.2_{-2.2}^{+3.0} \mathrm{eV}$ for $\mathrm{BaO}^{+}$. These values are well within the 0 to $100 \mathrm{eV}$ range usually observed.

\subsubsection{Charge Density Effect}

As the ion cloud grows in size, the ToF spectrum is expected to be smeared due to the high charge density of the cloud. One can expect ions in front of the 


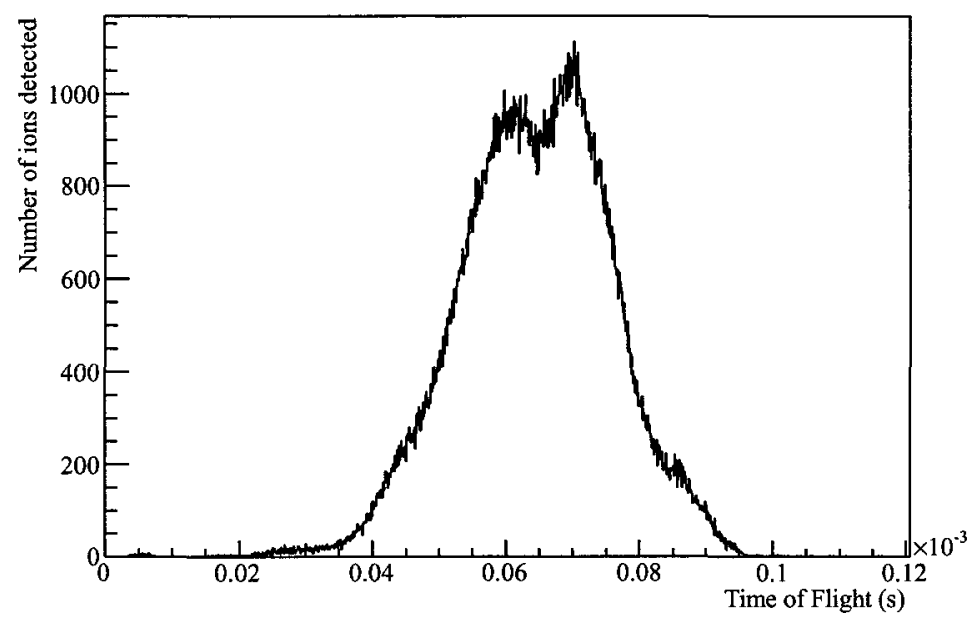

Figure 4.18: Time of Flight spectrum from kinetic energy only by laser ablation of barium metal.

cloud to be pushed forward and arrive earlier than if they were accelerated only by the drift field. Similarly, an ion at the back of the cloud would be slowed down, making a single mass peak broader than expected. Simulations of an ion cloud is computationally demanding, considering that for each time step one needs to calculate the force on each individual ion. For $n$ ions, there are $\frac{n^{2}}{2}$ calculations to be made per time step. Therefore, for ion populations in the $10^{4}-10^{8}$ range, one has to use charge renormalization to reduce the time of simulations. The one used in this study was to simulate an ion cloud interacting and multiplying their charge by a factor. That way, ions are bunched in smaller groups and an approximate simulation can be performed.

Using SIMION, the ToF spectra of $10^{7} \mathrm{Ba}^{+}$ions was simulated with and without electrical interaction in the cloud. The resulting ToF spectra are depicted in Figure 4.19. The mean of the distribution seems to shift to longer ToF and the width is definitely larger when interactions are activated. Figure 4.20 shows the location and the width of the mass peak from ion clouds made of 1 to $10^{8}$ ions. 
There is a clear threshold at $10^{6}$ ions when interactions seem to change the distribution. The time of arrival of ions is retarded and smeared as the number of ions is increased. Therefore, the laser power should be kept at a minimum to reduce any smearing of the mass spectra. This is only an approximation since the ion kinematics strongly depends on the geometry of the plume of ions produced in the first few nanoseconds which is not modelled in SIMION. A very tight plume would have a more explosive behaviour than a looser one. Using the measurement of the charge induced on the cathode, it has been estimated that between $10^{4}$ and $10^{7}$ ions per pulse were produced from the laser ablation source. In order to keep the CEM from saturating, the lower number of ions were used. It is therefore safe to assume that the high charge density effect is negligible in the data.

\subsubsection{Barium Mass Spectrum Analysis}

Several barium spectra were taken for different drift voltages. A spectrum example is shown in Figure 4.21. The source was a pure barium metal shard and the drift voltage was $1000 \mathrm{~V}$. Different lines are visible in the spectrum. Masses 137 and $153 \mathrm{Da}$ are the dominant peaks and represent $\mathrm{Ba}^{+}$and $\mathrm{BaO}^{+}$. Other unidentified lines are masses 44, 88, 106 and a large distribution around 310 Da.

\subsubsection{Chamber Drift Constant}

The drift constant $A$ for the chamber can be found by looking at the position of a known mass line as a function of the drift voltage. Figure 4.22 shows the ToF of barium ions for different voltages and a linear fit is used to give the constant $A$. For $\mathrm{Ba}^{+}$, from 1000 to $2000 \mathrm{~V}$, this constant is $24.1 \pm 1.5 \mu \mathrm{s}(\mathrm{qV} / \mathrm{m})^{1 / 2}$. The $A$ constant found from SIMION simulations is $22.8 \mu \mathrm{s}(\mathrm{qV} / \mathrm{m})^{1 / 2}$, within the uncertainty of the experimental measurement. This constant is different than the one calculated from simulations in Section 4.6.1 of $19.53 \mu \mathrm{s}\left(\mathrm{E}_{K} / \mathrm{m}\right)^{1 / 2}$, for equivalent energies. This can easily be explained by irregularities in the electric field or by a slow acceleration 


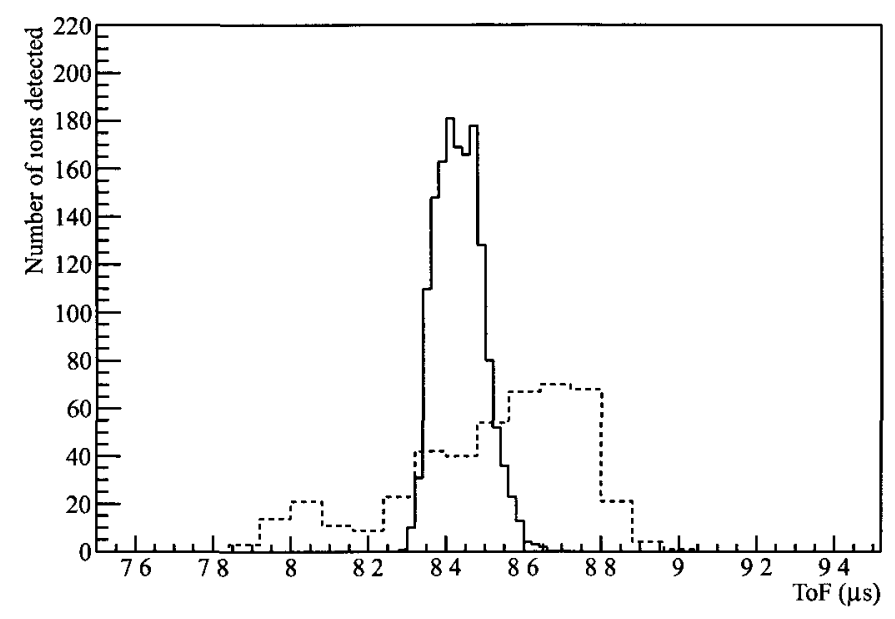

Figure 4.19: Simulated ToF spectra showing the effect of interactions in the ion cloud on the ToF spectrum resolution. The solid line shows the ToF spectrum of $\mathrm{Ba}^{+}$ions propagated without electrical repulsion and the broken one shows the ToF for a cloud of $10^{7}$ ions with the repulsion activated.

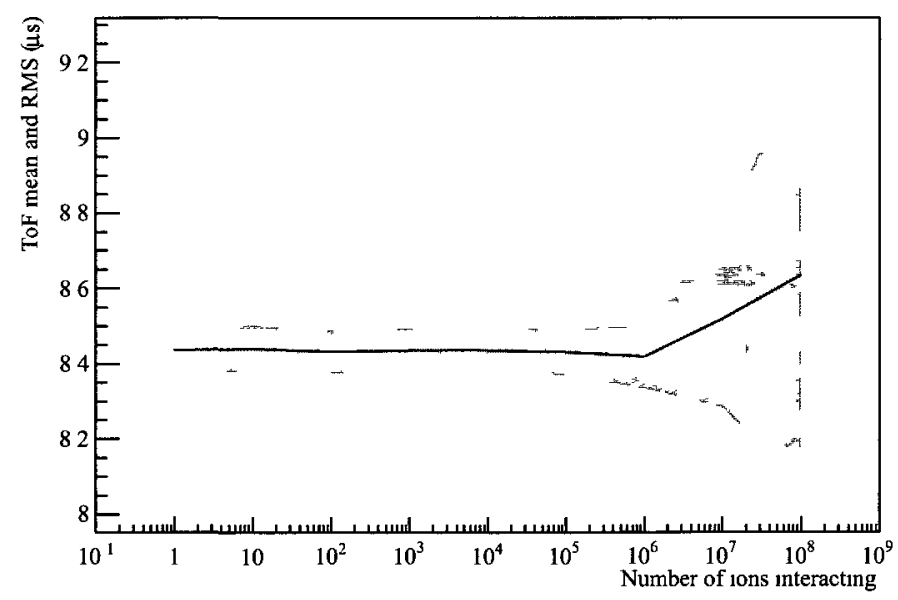

Figure 4.20: Mean and RMS of the simulated ToF distribution as a function of the number of ions in the cloud. The mean is represented by the line and the RMS of the distribution is shown by the gray area. 


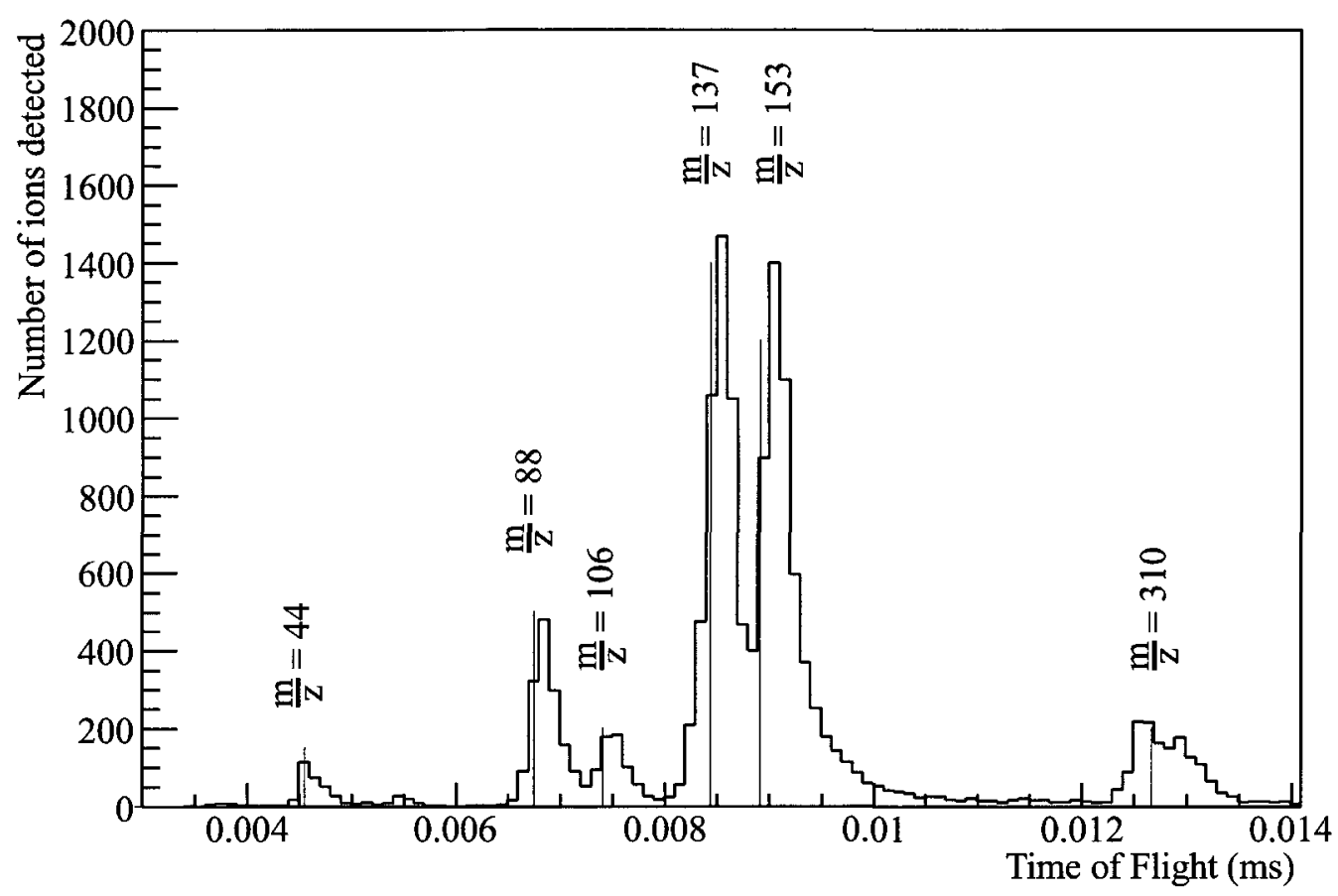

Figure 4.21: Mass spectrum from laser ablation of a pure Ba target. The histogram is the data recorded and the thin lines are the simulated masses of 44, 88, 106, 137, 153 and $310 \mathrm{Da}$. The intensity of the simulated peak is arbitrary. Masses of 137 and $153 \mathrm{Da}$ are from $\mathrm{Ba}^{+}$and $\mathrm{BaO}^{+}$respectively, while others are not known. 


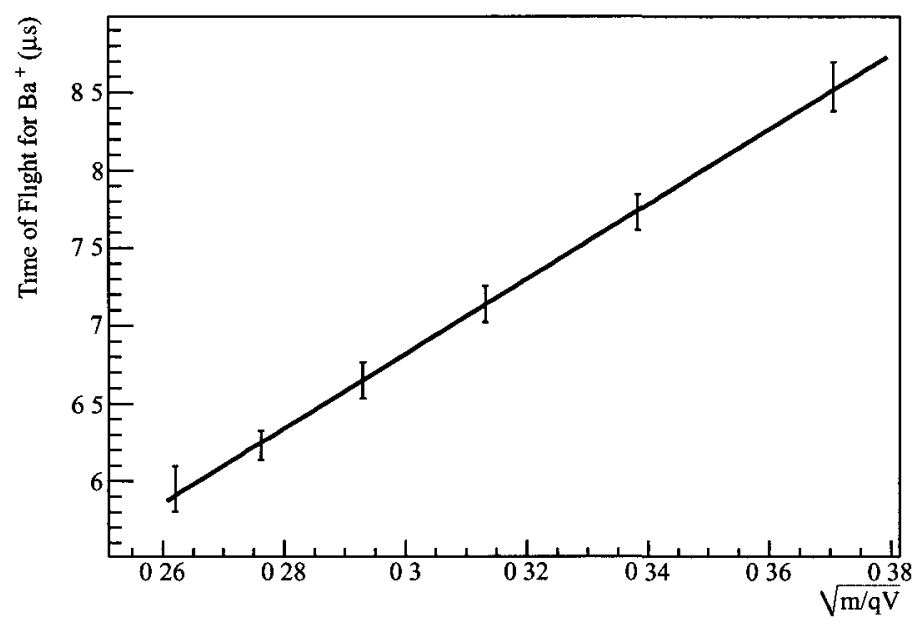

Figure 4.22: Barium line position as a function of the drift voltage. The error bars are the RMS of the $\mathrm{Ba}^{+}$peak.

of the cloud in the gas. If only $73 \%$ of the electric field serves to accelerate the ion both values would be equal.

Using the constant $A$ from Figure 4.22 , the time of flight for ${ }^{137} \mathrm{Ba}^{+}$is $8.920 \mu \mathrm{s}$ while for ${ }^{138} \mathrm{Ba}^{+}$it would $8.953 \mu \mathrm{s}$, for a difference of $32 \mathrm{~ns}$. The RMS of the time distribution is of the order of $100 \mathrm{~ns}$. This width is only due to the initial kinetic distribution and the initial trajectory angles. This means that the chamber is unable to discriminate individual isotopes of barium, but has a great discrimination efficiency for other atoms or molecules produced. The idea behind this simple mass spectrometer was to identify the products of the laser ablation technique. It is obvious that the laser ablation technique generates $\mathrm{Ba}^{+}$in large quantities which can be used for the fluorescence study. Some other lines were unidentified, but there was no sign of $\mathrm{Ba}^{++}$at $\mathrm{m} / \mathrm{z}=68.5 \mathrm{Da}$. 


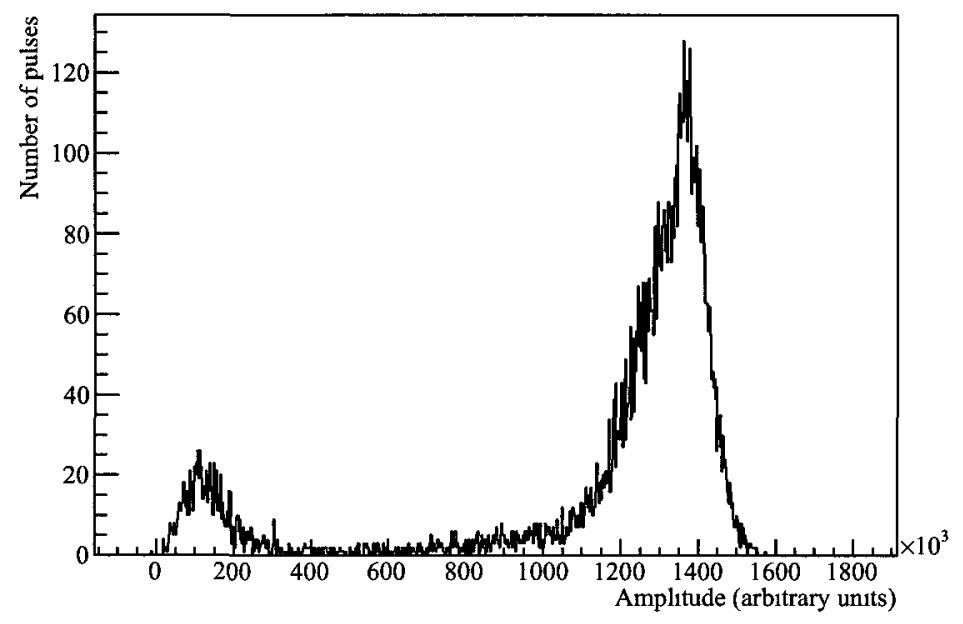

Figure 4.23: Charge collection intensity spectrum for a typical run in vacuum from ions generated from laser ablation.

\subsection{Laser Ablation Ion Fluorescence}

The chamber required some modifications to enable a laser fluorescence measurement. Electric field shaping rings were added and ports were installed to allow a laser beam to enter and exit the chamber. A lens was also installed to focus the fluorescence light onto an external PMT. The ion fluorescence apparatus is depicted in Figure 4.24. Detection of fluorescence from ions generated from laser ablation was more difficult than expected. The number of ions detected from one run to the other varied greatly, mainly due to the decay problem described in Section 4.4.2, but also due to the pulse to pulse variation. This variation is extreme as can be seen in Figure 4.23. One laser pulse can produce no ions while the next one could produce more than $10^{6}$.

A charge collection measurement was required to normalize the fluorescence signal. To record the signal, a computer sound card was used. It was a cheap and high performance option due to the long arrival time of ions in gas (few ms) and 
the fast repetition rate of the laser $(10$ to $100 \mathrm{~Hz}$ ). The ASUS Xonar D1 sound card can record 2 channels at a $192 \mathrm{kHz}$ sampling rate and a 24 bit resolution with a maximum range of $3 \mathrm{~V}$ peak to peak. To match the impedance of the sound card and to give more flexibility to record the signal, the charge induced was amplified by an amplifier with a gain of between 0.1 and $10 \times$. The charge signal was recorded as an uncompressed .wav file with two channels, one for the charge collected and the other for the trigger pulse coming from the laser. Besides the charge collected information, the laser wavelength was also recorded every second in a file since the laser wavelength could vary within a few minutes going from on resonance to off resonance. The fluorescence signal was also detected with a photomultiplier tube (PMT), the Hamamatsu photosensor module H7422P-40, and recorded with the MCS described in Section 4.5. The PMT had three filters to reduce the intensity of scattered light. The first one was a UV filter to reduce the light from the ablation laser, the second was a bandpass filter to eliminate the scattered $493 \mathrm{~nm}$ laser light and the last one is a line filter to let only $650 \mathrm{~nm}$ light reach the PMT. The PMT was operated in counting mode, with a discriminator set for single photon sensitivity. Finally, other pertinent information was written by hand in the logbook, then included in a single file with all the information discussed above, including the charge collection signal. The data was then read and analyzed with the ROOT software. This software provides a framework in $\mathrm{C}++$ allowing to store, analyze and display data.

Figure 4.25 shows the charge induced in the cathode by ions propagating in vacuum and in a small amount of gas. Figure 4.25a and Figure 4.25b should, in theory, be very similar. The amount of gas does not explain the broad distribution in arrival time in Figure 4.25b, unless the gas helps to form a large cluster of ions. This could also explain the difficulty to see fluorescence when gas is added in the chamber. A large gas leak was found afterwards in the gas handling system, making it probable that nitrogen, oxygen and water vapour was introduced in the chamber with the argon. 


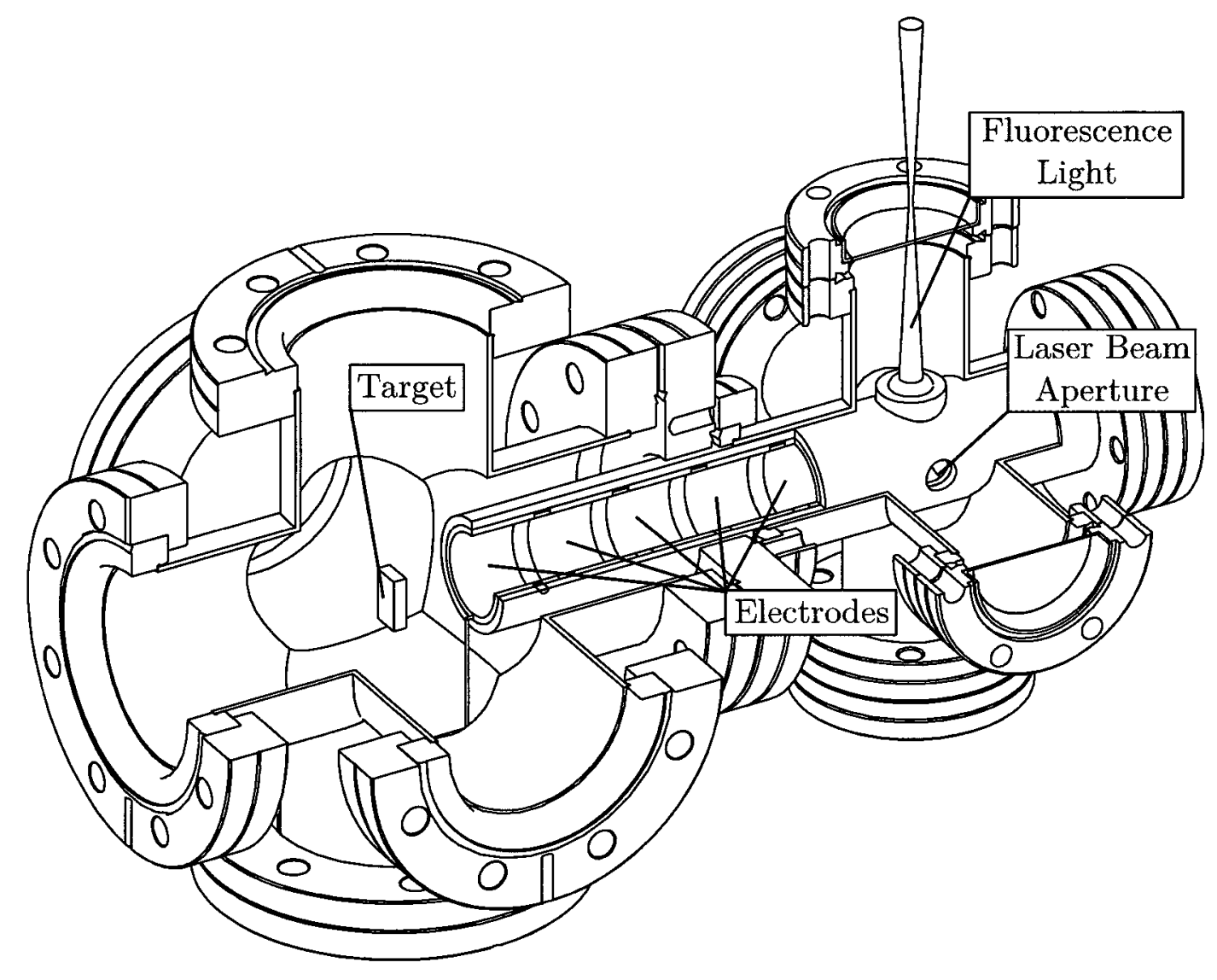

Figure 4.24: Fluorescence chamber internal schematic. 


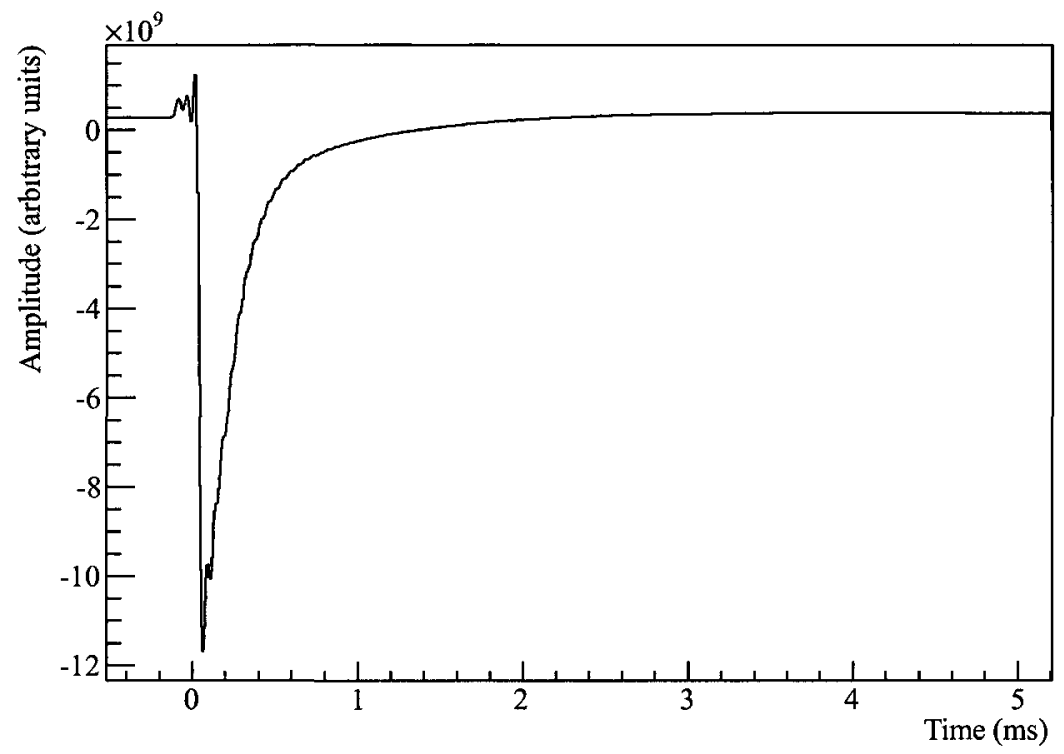

(a)

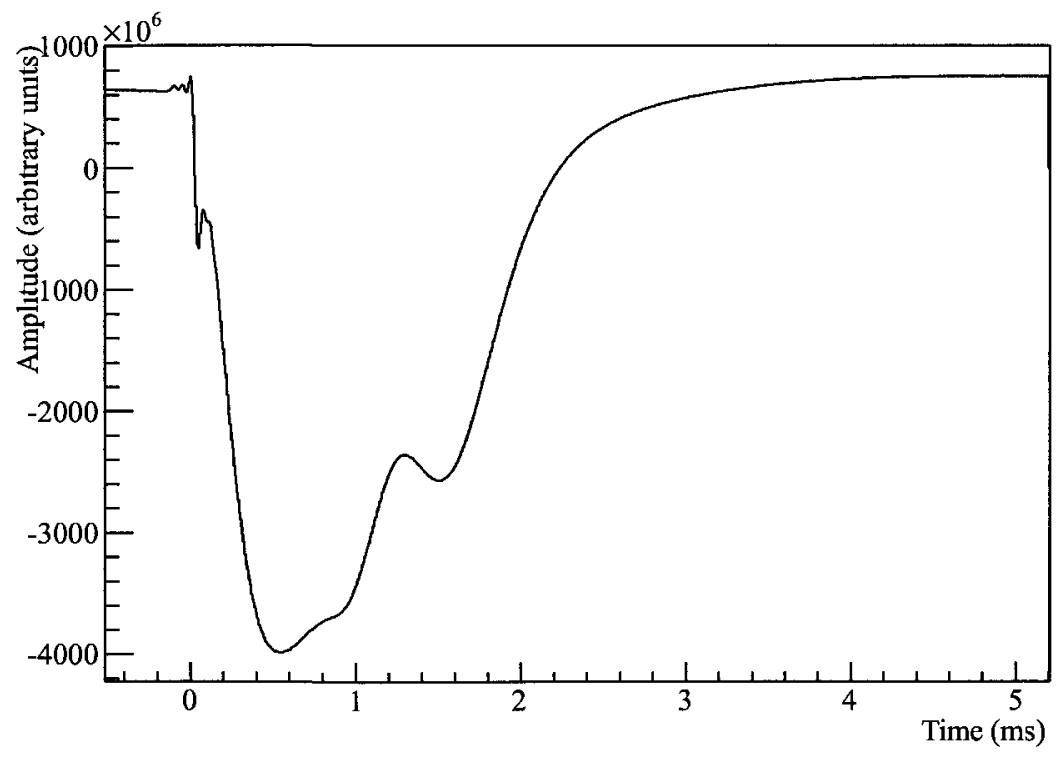

(b)

Figure 4.25: Average trace recorded on the cathode of ions emitted from laser ablation. (a) was taken in vacuum and (b) was recorded at pressure around 0.1 mbar of argon. 


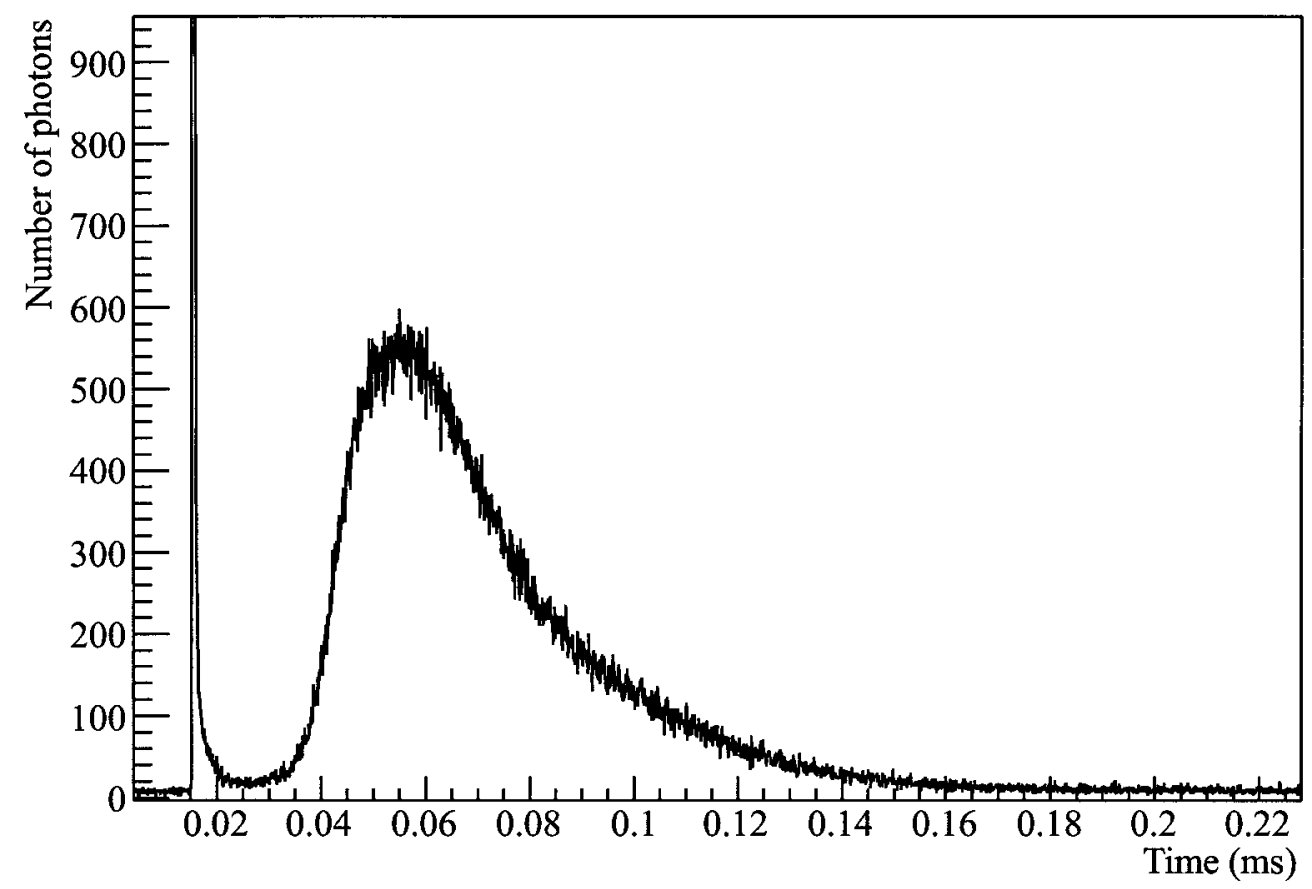

Figure 4.26: Fluorescence time spectrum at a pressure of $0.8 \mathrm{mbar}$ of Ar. The narrow peak on the left is due to the light from the ablation laser and the large distribution at $0.06 \mathrm{~ms}$ is the fluorescence. About 235,000 photons were detected for 60,494 laser pulses. Assuming a quantum efficiency of $30 \%$ for the PMT and a coverage of $2 \%$, the number of generated $650 \mathrm{~nm}$ photons per ion cloud was about 650 .

A fluorescence time spectrum is shown in Figure 4.26. It shows the number of photons detected by the PMT in vacuum, after 60,494 laser ablation pulses. The first large and narrow peak is the light detected from the ablation laser. The broader distribution is the $650 \mathrm{~nm}$ fluorescence from $\mathrm{Ba}^{+}$when probed by $493 \mathrm{~nm}$ laser light. When the laser wavelength was shifted by a tiny amount, this fluorescence disappeared. 


\subsubsection{Ion Drift}

At a pressure of $1 \mathrm{~atm}$ of $\mathrm{Xe}$, the $\mathrm{Ba}^{+}$diffuses very slowly. The diffusion length $(D)$ is calculated with:

$$
D=L \sqrt{t / \tau}
$$

with $t$ the time elapsed, $\tau$ the time between collisions and $L$ the mean free path of the ion. $L$ is given by:

$$
L=\frac{1}{k\langle v\rangle N}
$$

with $k$ the rate constant from Equation 4.17, $\langle v\rangle$ the mean velocity of ions and $N$ the number density. The mean velocity is taken from a Maxwell-Boltzmann distribution:

$$
\langle v\rangle=\sqrt{\frac{8 R T}{\pi M}}
$$

where $R$ is the gas constant, $T$ is the temperature and $M$ is the molar mass. For $\mathrm{Ba}^{+}$at $300 \mathrm{~K},\langle v\rangle=216 \mathrm{~m} / \mathrm{s}$. Going back to Equation 4.44, the mean free path of a barium ion becomes $15.0 \mathrm{~nm}$ at $1 \mathrm{~atm}$. As for the collision rate, Section 4.1.4 shows that at $1 \mathrm{~atm}$ it is $1.43 \times 10^{10} \mathrm{~s}^{-1}$, which gives a time of $70 \mathrm{ps}$ between collisions. Finally, using Equation 4.43, the diffusion distance is $1.8 \mathrm{~mm}$ for a duration of $1 \mathrm{~s}$. Assuming a $1 \mathrm{~m}$ TPC with an electric field giving a drift velocity for electrons of $0.1 \mathrm{~cm} / \mu \mathrm{s}$, electrons would drift for a maximum of $1 \mathrm{~ms}$ before the topology of the tracks could be measured. Meanwhile, the $\mathrm{Ba}^{+}$would have diffused for $56.7 \mu \mathrm{m}$ and simulations with SIMION showed that it would have drifted in the opposite direction by $35 \mathrm{~mm}$.

The drift velocity was recorded using the fluorescence signal in $0.8 \mathrm{mbar}$ and 2.5 mbar of argon with a field of $500 \mathrm{~V}$ over $200 \mathrm{~mm}$. The ToF at $0.8 \mathrm{mbar}$ was 
$0.44 \mathrm{~ms}$ and at $2.5 \mathrm{mbar}$ it was $1.40 \mathrm{~ms}$. So, this translates into a drift velocity of 14.4 and $14.3 \mathrm{mbar}(\mathrm{cm} / \mathrm{s})(\mathrm{V} / \mathrm{cm})^{-1}$. An uncertainty of $3.9 \mathrm{mbar}(\mathrm{cm} / \mathrm{s})(\mathrm{V} / \mathrm{cm})^{-1}$ can be assigned to this number due to the uncertainty in the electric field. This value is taken from the discrepancy between the two $A$ constants described in Section 4.6.4.

\subsubsection{Linewidth and Position}

The linewidth and position of the $493 \mathrm{~nm}$ barium line was measured again using a procedure different from than in Section 4.3.1. The other barium lines were not probed to record the amount of $\mathrm{Ba}^{+}$produced, so the intensity was taken from the charge collection using the sound card. The blue laser wavelength was scanned and the intensity of the $650 \mathrm{~nm}$ line was recorded as the fluorescence signal. The signal was normalized to the amount of ions collected and to the intensity of the blue laser. The blue laser intensity was measured in two ways. One with an external power meter and the second one, using the background trigger rate of the PMT when no ions were produced. Both ways were equivalent as shown in Figure 4.27.

The fitted position was $493.5462 \mathrm{~nm}$ while the expected position is $493.5454 \mathrm{~nm}$. The difference of $+0.0008 \mathrm{~nm}$ is half that measured in $30 \mathrm{mbar}$ of gas, so this difference is a good estimation of the uncertainty on the laser wavelength. The width can be measured by fitting a Gaussian, since most of the linewidth should be related to the Doppler broadening. This time, no uncertainty was assigned to this type of broadening. The measurement was initially performed in vacuum, so there was no gas to cool down ions and the temperature could have been as high as 50,000 K. Subsequently, the ion cloud should cool by expelling ions with large transverse velocities. The width was found to be $2.13 \pm 0.06 \times 10^{-3} \mathrm{~nm}$, much broader than the width measured in 30 mbar of gas. Assuming that most of the

width comes from the Doppler broadening, the temperature of the cloud at the fluorescence location can be inferred to be $5000 \mathrm{~K}$. 


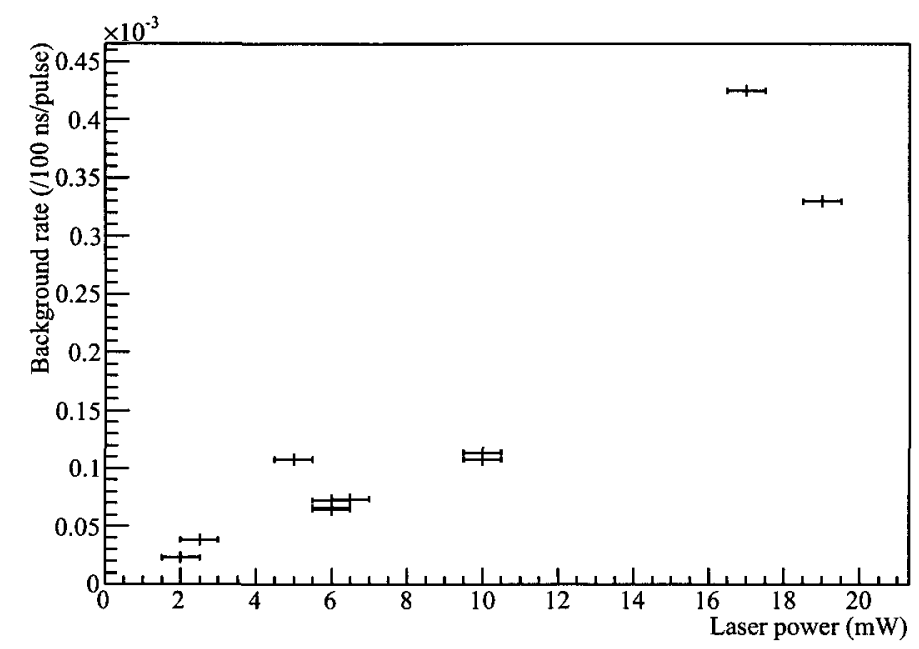

Figure 4.27: Correlation between blue laser intensity and background counting rates.

Again, the number of ions generated by the laser ablation ion source was too unstable for the barium tagging study. Moreover, the Coherent Sabre Laser that was used to pump the $493 \mathrm{~nm}$ dye laser suffered from repetitive failures, yielding very slow progress in the barium tagging procedure in high pressure gas. The idea of adding the electron donor gas to the xenon to perform the barium charge conversion was a problem if the EL signal was to be measured. Therefore, the effort for barium tagging was diverted to extracting and converting the ion outside the xenon volume. Further work on barium tagging in high pressure gas is no longer a priority and has been removed from the research program. Meanwhile the laser has been repaired and may be used for other purposes. 


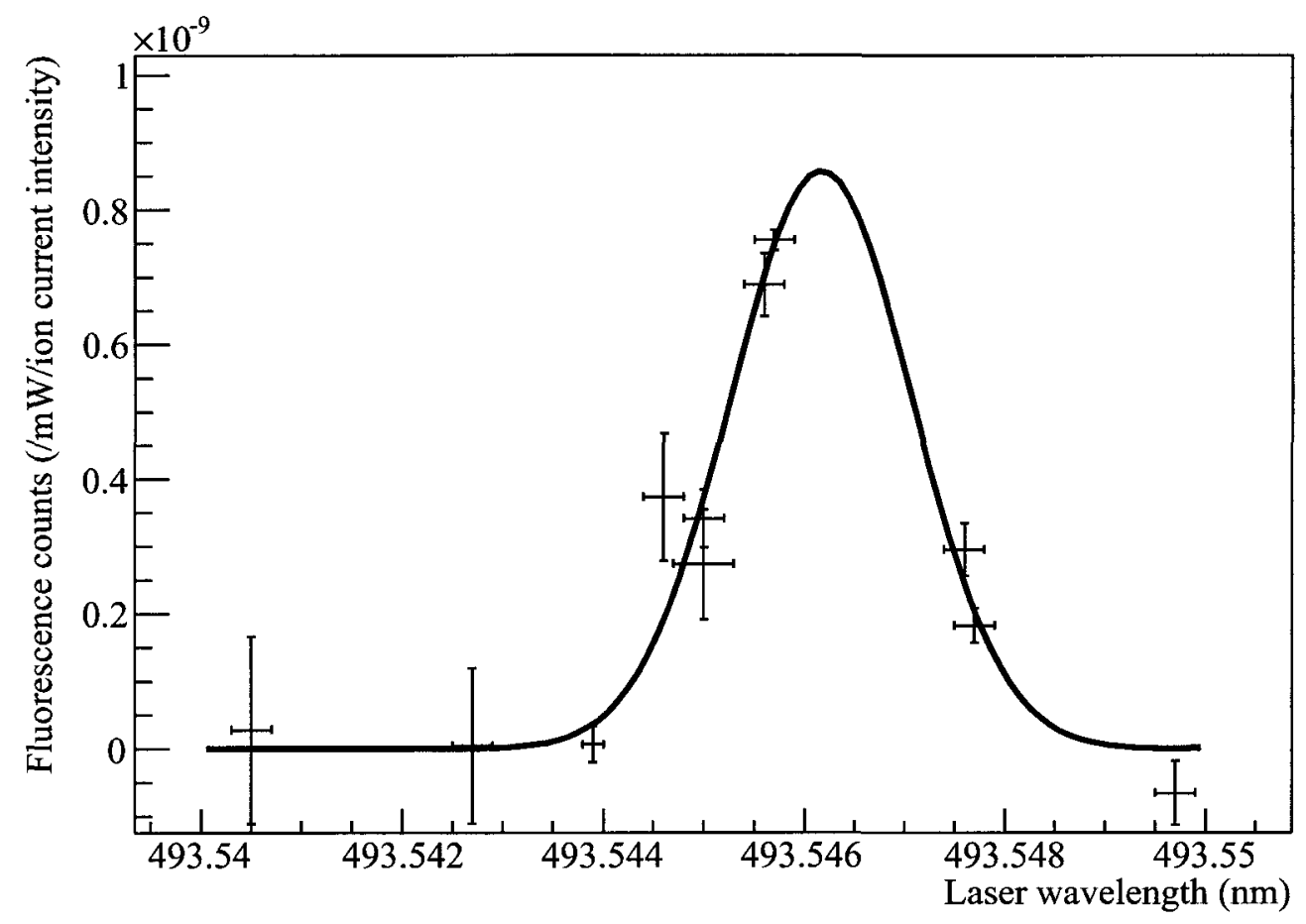

Figure 4.28: Line profile of the $S_{1 / 2}-P_{1 / 2}$ transition in vacuum with ions from the laser ablation apparatus. 


\section{Chapter 5}

\section{Doubly Charged Barium Ion Extraction and Charge Conversion}

The $0 \nu \beta \beta$ decay investigated by EXO offers the unique possibility of detecting the barium daughter of the reaction. The LIF technique relies on the fact that $\mathrm{Ba}^{++}$is converted into $\mathrm{Ba}^{+}$by acquiring an electron from a surrounding molecule. The electron exchange could be performed inside the $0 \nu \beta \beta$ volume, but doing it outside would be preferable for two reasons. First, the main $0 \nu \beta \beta$ volume would be less prone to contamination by the electron donor gas. Secondly, the parameters could be more precisely controlled to optimize the transfer between the gas and the ion. By carefully adjusting the pressure and the electric field in the vicinity of the $\mathrm{Ba}^{++}$, one can control the number of collisions and the collision energy.

In a xenon gas $0 \nu \beta \beta$ decay experiment, the tagging procedure could be as follow. Since a $\mathrm{Ba}$ ion is 27,000 times heavier than an electron, its drift velocity is considerably slower and there is no problem to wait until the tracks are reconstructed before extracting the ion. The two primary electron tracks would locate the $\mathrm{Ba}^{++}$in the $\mathrm{x}-\mathrm{y}$ plane and the drift time would locate it in the $\mathrm{z}$ direction. 
The ion could be drifted toward an orifice and then extracted to an intermediate pressure region, inside a hexapole ion guide (SPIG) where the ion is confined in the centre and the uncharged gas is pumped out of the system. The ion would then collide with an electron donor gas to convert it to $\mathrm{Ba}^{+}$. Finally the ion could be stored in a linear quadrupole trap and the fluorescence measurement could be performed.

Some concepts have to be demonstrated before implementing the tagging procedure in a gas phase experiment. First of all, to efficiently extract the $\mathrm{Ba}^{++}$using an electric field, its mobility has to be measured since it is currently unknown. This thesis does not cover this aspect, but an apparatus is being built at Carleton University to measure it. It is also important to measure the efficiency to extract the ion from high pressure gas to vacuum and to confine it in a trap. Part of this procedure is a proven technology. The extraction from gas to vacuum in a hexapole ion guide is readily done, as will be shown in Section 5.1.1, although the efficiency is unknown. Also, loading an ion trap from an ion guide is also a proven technology and the concept is used in some modern mass spectrometers. The total efficiency measurement is planned and will be performed at a later time. It is imperative that $\mathrm{Ba}^{++}$does not form a molecule with the surrounding gas, otherwise LIF is impossible. Section 5.1.1 covers this concept. Finally, tagging in a trap has already been demonstrated by the EXO collaboration [43].

\subsection{Triple Quadrupole Mass Spectrometer}

Tandem mass spectrometry (MS/MS) provides a means to test some of these concepts. In a MS/MS instrument, ions go through a mass spectrometer, a collision cell and a second mass spectrometer. Both mass spectrometers can be set as nonfiltering ion guides or to act like mass filters or to scan the mass spectrometer range to record a mass spectrum. The collision cell can be filled with gas and an electric field can be applied to accelerate the ions. Comparing the compounds 


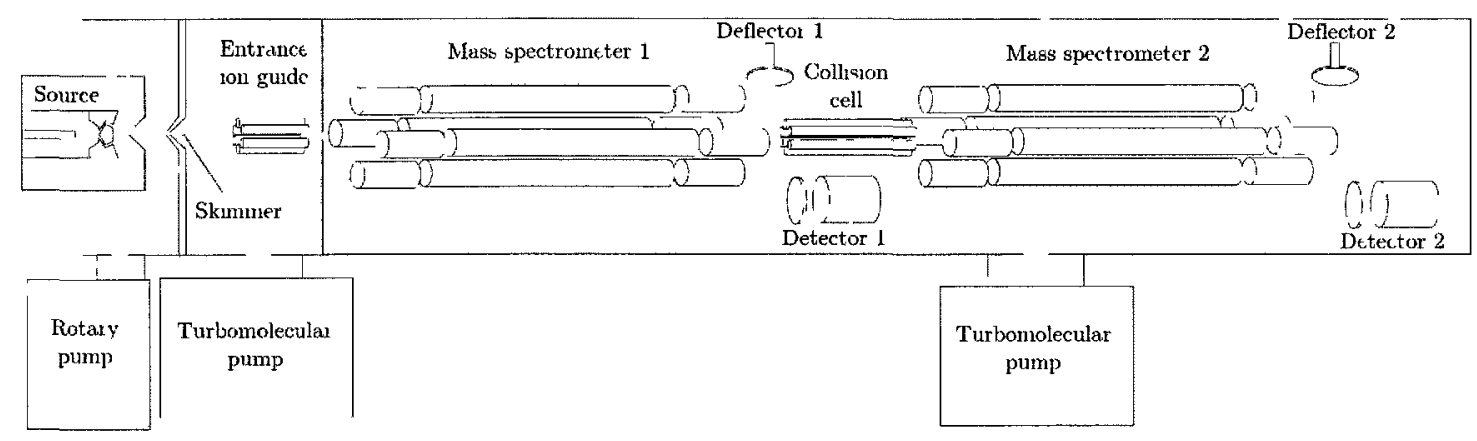

Figure 5.1: Quattro mass spectrometer schematic.

before and after the collision cell as a function of the gas type, its pressure and the kinetic energy of ions, it is possible to measure the rate and the cross-section of the reactions.

The instrument used is a Quattro II quadrupole-hexapole-quadrupole mass spectrometer (QhQ/MS) sold by Waters. Ions enter the instrument through a $0.2 \mathrm{~mm}$ aperture, and then are confined in a SPIG to allow the neutral gas to be pumped away. The ion continues through the first quadrupole guide that can be used as a mass filter and the ion signal is measured by a Dynolite ion detector. The hexapole is located inside a $16 \mathrm{~cm}$ long collision cell that can be filled with gas at a pressure between $10^{-4}$ to $10^{-2}$ mbar. Ions entering the collision cell can be accelerated by an electric field generated by a potential between 0 and $250 \mathrm{~V}$ at the entrance of the cell. The second quadrupole ion guide is placed after the collision cell and monitors the fragments generated inside the collision cell. Ions can be diverted to the ion detector right after the first mass spectrometer using a deflector plate. If the deflector is not activated, ions continue their way to the rest of the unit. The instrument is outfitted with two turbomolecular pumps backed by a rotary vane pump. The different parameters are controlled from a computer. Several ion sources can be mounted, but only the electrospray source has been used in this study. Figure 5.1 shows the schematic of the instrument. 


\subsubsection{Electrospray Ion Source}

$\mathrm{Ba}^{++}$can be produced using an electrospray source. The mechanism to produce ions from an electrolyte solution is elegant. A solution containing barium salt is pushed through a capillary tube by a syringe pump. The capillary is inside a second small tube carrying a gas, typically nitrogen, to nebulize the liquid and produce small droplets. A high voltage at the tip of the capillary charges the droplets and a drying gas, nitrogen again, flows to direct them through an aperture. The drying gas also scatters large droplets and helps to evaporate some of the solvent. As solvent evaporates, the charge density increases and when the Coulomb field inside the droplets is sufficient, ions inside the droplets are desorbed from it. Figure 5.2 shows schematic of the electrospray source.

The liquid used is a solution of barium acetate, $\mathrm{Ba}\left(\mathrm{CH}_{3} \mathrm{COO}\right)_{2}$, at a $1 \mathrm{mM}$ concentration in a 50:50 mixture of water and methanol. It is pushed in the system at a rate of $1 \mathrm{~mL} / \mathrm{h}$ using a syringe pump. Different parameters can be tuned to get different ion types. For example, a high sample cone voltage is known to be very efficient to break long molecules. A typical barium acetate spectrum is shown in Figure 5.3.

The parameters of the source and mass spectrometer were optimized to increase the production of $\mathrm{Ba}^{++}$and reduce other components of the spectrum. Optimal parameters are listed in Table 5.1. The nomenclature of the Quattro II control software differs from the instrument user manual. The capillary voltage is applied on the sample inlet, in which the solution flows, in order to charge the droplets. On the electrospray source side, the HV lens is applied on the counter electrode and the cone voltage is applied on the sample cone. On the mass spectrometer side, the skimmer voltage is applied on the skimmer element and the RF Lens is in fact the entrance ion guide. All Lens voltages are related to pre-filters before and after the two mass spectrometers to help to discard clusters of molecules. IEnergy voltage bias the voltage at the entrance of each mass spectrometer to supply ions with some energy. Finally, Mult voltage is the voltage applied on the two PMTs 


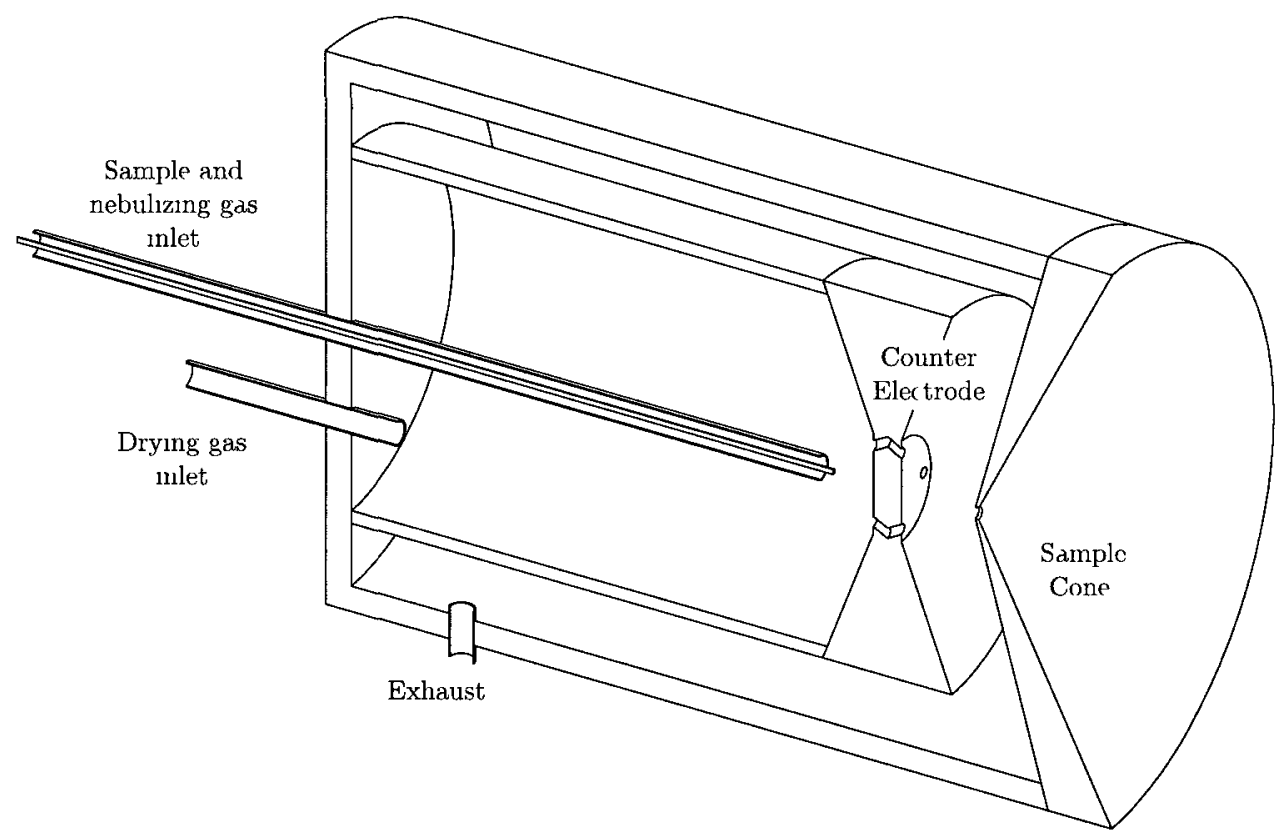

Figure 5.2: Electrospray source schematic. The liquid in inserted in the small capillary tube, in the centre. Nebulizing gas flows in a larger tube around the capillary and heated drying gas flows inside the source. Ions go across the counter electrode through one of four elbow shaped apertures. They leave the source through the sample cone aperture. The whole system is about $30 \mathrm{~cm}$ long. 


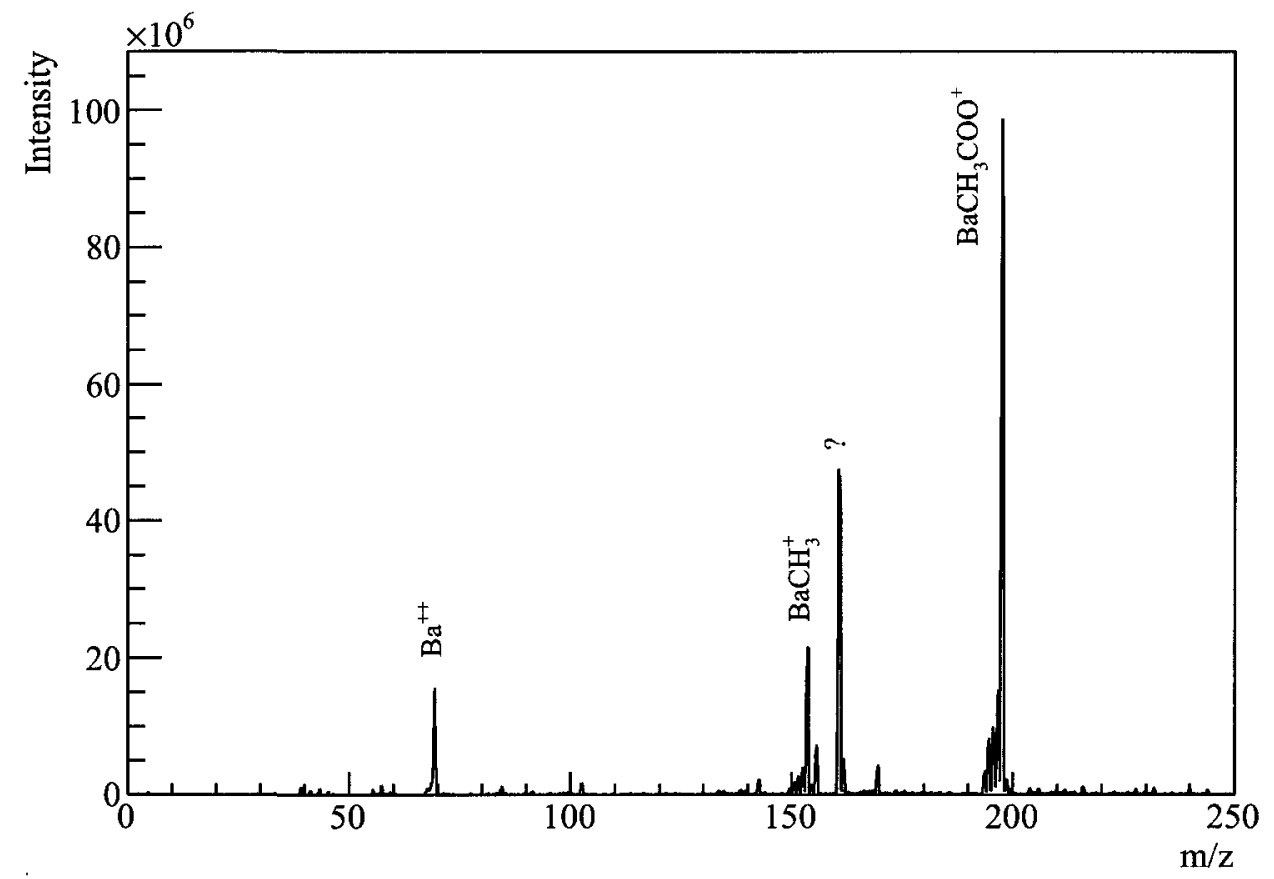

Figure 5.3: Typical barium acetate mass spectrum from the electrospray source. 
Source

\begin{tabular}{|c|c|}
\hline Capillary & $2.73 \mathrm{kV}$ \\
\hline HV Lens & $0.08 \mathrm{kV}$ \\
\hline Cone & $60 \mathrm{~V}$ \\
\hline Skimmer & $1.6 \mathrm{~V}$ \\
\hline RF Lens & 0.5 \\
\hline
\end{tabular}

MS1

\begin{tabular}{|c|c|}
\hline LM Res & 14 \\
\hline HM Res & 14 \\
\hline IEnergy 1 & $1.8 \mathrm{~V}$ \\
\hline Lens 6 & 10 \\
\hline Mult 1 & $750 \mathrm{~V}$ \\
\hline
\end{tabular}

MS2

\begin{tabular}{|c|c|}
\hline LM Res & 14 \\
\hline HM Res & 14 \\
\hline IEnergy 2 & $1.1 \mathrm{~V}$ \\
\hline Lens 8 & 345 \\
\hline Lens 9 & 50 \\
\hline Mult 2 & $750 \mathrm{~V}$ \\
\hline
\end{tabular}

Table 5.1: Parameters for the electrospray source and mass spectrometer to optimize the production of $\mathrm{Ba}^{++}$.

measuring the scintillation light given by the ion detectors.

The liquid is nebulized using nitrogen gas flowing at a rate of $10 \mathrm{~L} / \mathrm{h}$ around the capillary where the liquid enters the source. The flow rate of the drying gas is $250 \mathrm{~L} / \mathrm{h}$. The source is heated up at $80^{\circ} \mathrm{C}$ to facilitate the evaporation.

A mass spectrum from the barium acetate electrospray source with parameters adjusted for a high $\mathrm{Ba}^{++}$output and a high mass resolution is shown in Figure 5.4. The main component of the spectrum is $\mathrm{Ba}^{++}$and other components are minor, at levels below $10 \%$. Table 5.2 shows the intensity of the different isotopes of $\mathrm{Ba}^{++}$ compared with their natural abundance. From that, and the fact that peaks are spaced by $1 / 2$ unit of $m / z$, there is no doubt about the origin of the $m / z=69$ peak.

Many surprising features are present in the source spectrum. First, $\mathrm{Ba}^{++}$is the main component of the spectrum, with $\mathrm{Ba}^{+}$at about $1 \%$ of the $\mathrm{Ba}^{++}$peak. This makes the electrospray source a perfect ion source to characterize the doubly charged barium ions produced by the $0 \nu \beta \beta$ decay. Second, even in the presence of water vapour, methanol and nitrogen, $\mathrm{Ba}^{++}$is still produced in large amounts. At an injection rate of $1 \mathrm{~mL} / \mathrm{h}$ of water and methanol and a drying gas flow rate of $250 \mathrm{~L} / \mathrm{h}$, it means that $1 \mathrm{~g}$ of liquid is evaporated in about $300 \mathrm{~g}$ of nitrogen gas each minute. Each $\mathrm{Ba}^{++}$has to go through nitrogen and a $0.3 \%$ mix of water and methanol vapour. Peaks of $\mathrm{BaCH}_{3} \mathrm{O}^{+}$and $\mathrm{BaOH}^{+}$are visible in the spectrum, at $5 \%$ of the intensity of $\mathrm{Ba}^{++}$, but these are not necessarily the products of the 


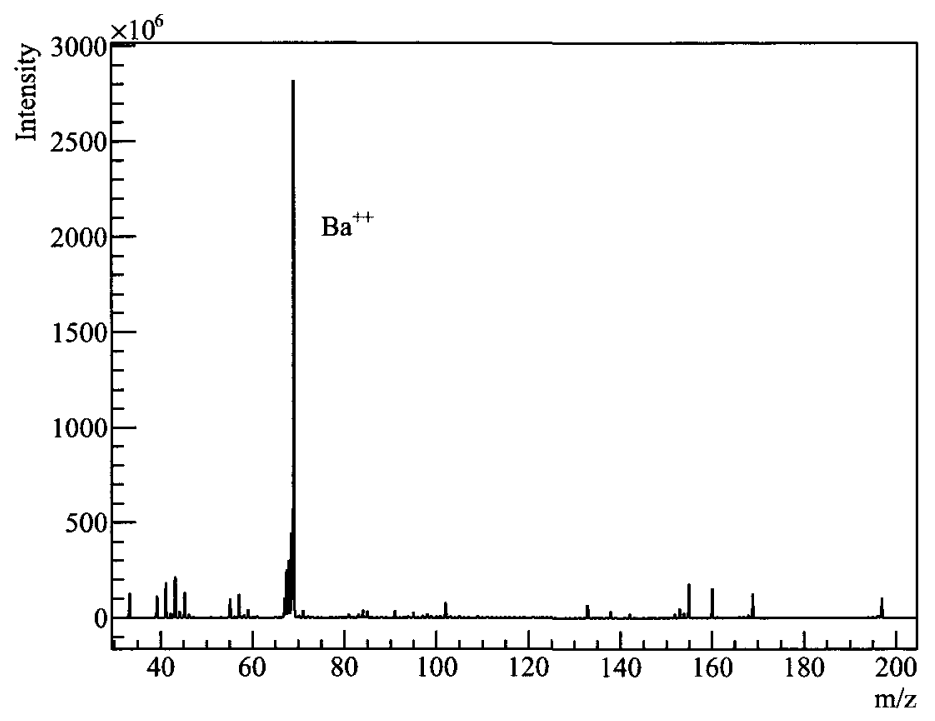

(a)

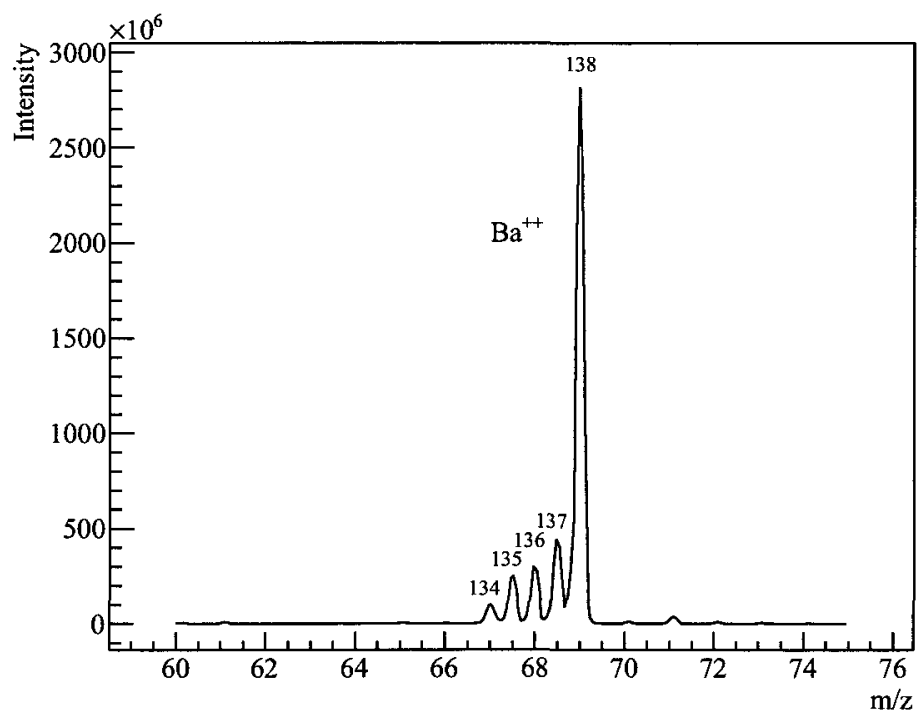

(b)

Figure 5.4: Electrospray source mass spectrum using barium acetate at a $1 \mathrm{mM}$ concentration in water and methanol 50:50. Parameters were optimized for an excellent mass resolution and to increase the $\mathrm{Ba}^{++}$detection. 


\begin{tabular}{|c|c|c|}
\hline Mass (Da) & $\begin{array}{c}\text { Natural Abundance } \\
(\%)\end{array}$ & $\begin{array}{c}\text { Measured Abundance } \\
(\%)\end{array}$ \\
\hline \hline 130 & 0.1 & 0.18 \\
\hline 132 & 0.1 & 0.07 \\
\hline 134 & 2.4 & 2.6 \\
\hline 135 & 6.6 & 6.4 \\
\hline 136 & 7.8 & 7.6 \\
\hline 137 & 11.2 & 11.3 \\
\hline 138 & 71.7 & 71.8 \\
\hline
\end{tabular}

Table 5.2: Barium isotope content in the $\mathrm{Ba}^{++}$peaks from the electrospray source. Intensities were measured from Figure 5.4b.

interaction between $\mathrm{Ba}^{++}$and water or methanol. They could come from the source itself when the droplets create ions. The fact that several $\mathrm{Ba}^{++}$ions survive means that they are relatively stable even in the presence of very reactive molecules. For $\mathrm{Ba}^{++}$drifting in pure xenon, a noble gas, the lifetime should be long enough to be able to drift it and extract it in a trap. Then, the charge exchange could occur and the tagging could be performed.

\subsubsection{Quadrupole Ion Guide}

Each mass spectrometer is built from four rods, on which both a direct current (DC) and a radio-frequency (RF) voltage are applied. Depending on the frequency and amplitude of the voltage applied on the rods, different mass to charge ratio $(\mathrm{m} / \mathrm{z})$ molecules will have different trajectories, some of them stable. These ions will be guided through the instrument while others will be discarded. Examples of SIMION simulations of stable and unstable trajectories are shown in Figures 5.5 and 5.6.

The rods are usually cylindrical, although to obtain a perfect quadrupole field, the cross-section of the rod should be hyperbolic. They are placed parallel to each other, at the four corners of a square. Rods are coupled in pairs, with opposing 

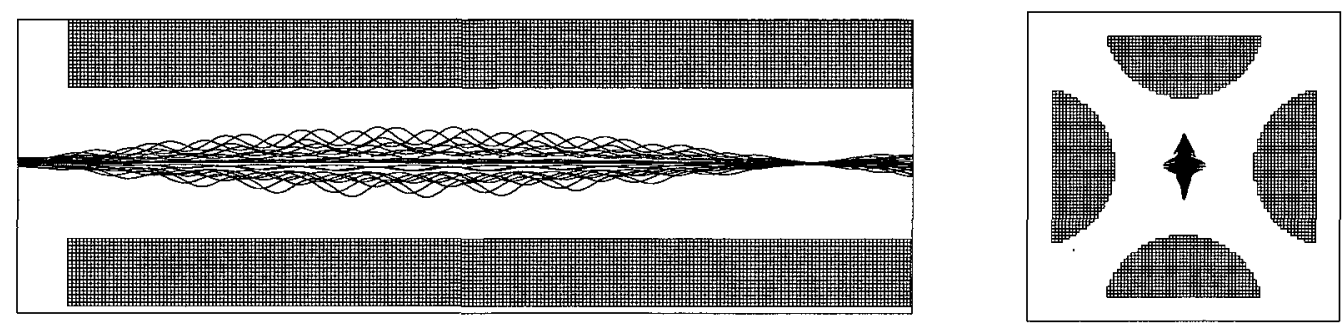

Figure 5.5: A SIMION simulation of $25 \mathrm{~m} / \mathrm{z}=100$ ions through a $50 \mathrm{~mm}$ long quadrupole. The frequency and amplitude of the RF and DC voltages were set to allow the transport of $m / z=100$ ions. The left diagram is a $\mathrm{x}-\mathrm{z}$ projection while the one on the right is the $\mathrm{x}-\mathrm{y}$ projection.
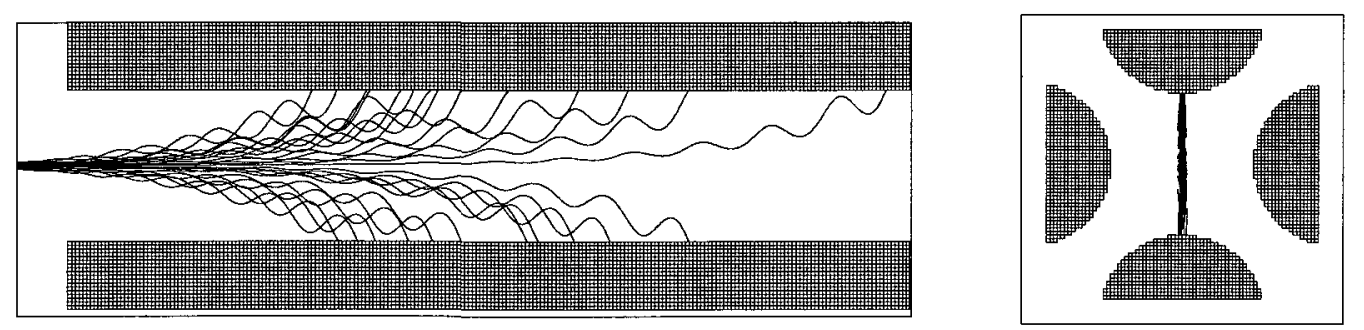

Figure 5.6: A SIMION simulation of $25 \mathrm{~m} / \mathrm{z}=120$ ions through the same quadrupole described by Figure 5.5 and under the same conditions. 
rods being at the same potential, while the other pair is of the inverse polarity.

The electromagnetic force that a charged particle feels within the quadrupole, perpendicular to the length of the guide and aligned with the centre of the guide, is:

$$
F_{u}=m \frac{d^{2} u}{d t^{2}}=-e \frac{\partial \phi}{\partial u},
$$

where $u$ is either the $x$ or $y$ direction, $z$ being the propagation direction and $m$ the mass of the particle. The electric potential $(\phi)$ is given by

$$
\phi=\frac{\phi_{0}}{r_{0}^{2}}\left(x^{2}-y^{2}\right) .
$$

Here, $\phi_{0}$ is the electric field applied to the rods and $r_{0}$ the half distance between the electrodes. The minus sign between the $x$ and the $y$ directions is due to the opposite polarity of the horizontal and vertical rods. As mentioned previously, the electric field can be a combination of $\mathrm{DC}$ and RF voltages. Therefore, $\phi_{0}$ is represented by

$$
\phi_{0}=U+V \cos (2 \pi f t)
$$

with $U$ and $V$ the $\mathrm{DC}$ and $\mathrm{RF}$ amplitude applied and $f$ the frequency in $\mathrm{Hz}$ of the RF signal. Now, taking the derivative of the potential and inserting it in Equation 5.1, the equation of motion in the $x$ direction becomes

$$
m \frac{d^{2} x}{d t^{2}}=\frac{-2 e}{r_{0}^{2}}(U+V \cos (2 \pi f t))
$$

The trapping parameters can be defined as

$$
a_{x}=\frac{8 e U}{m\left(2 \pi f r_{0}\right)^{2}}
$$


and

$$
q_{x}=\frac{-4 e V}{m\left(2 \pi f r_{0}\right)^{2}}
$$

with $a_{y}=-a_{x}$ and $q_{y}=-q_{x}$. Finally, substituting $\xi=\pi f t$, the Mathieu equation is obtained, again using $u$ for either the $x$ or $y$ direction, as

$$
\frac{d^{2} u}{d \xi}+\left(a_{u}-2 q_{u} \cos 2 \xi\right) u=0
$$

This equation is very difficult to solve but the Handbook of Mathematical Functions book by Abramowitz and Stegun [65] lists functions that define stability regions in the $a-q$ plane. Four of those regions exists, but usually the first, at low $a$ and $q$, is the most practical one to use. Its boundaries are defined by two functions:

$$
\begin{aligned}
& a_{0}=-\frac{q^{2}}{2}+\frac{7 q^{4}}{128}-\frac{29 q^{6}}{2304}+\frac{68687 q^{8}}{18874368}+\ldots \\
& a_{1}=1-q-\frac{q^{2}}{8}+\frac{q^{3}}{64}-\frac{q^{4}}{1536}-\frac{11 q^{5}}{36864}+\frac{49 q^{6}}{589824}+\ldots
\end{aligned}
$$

Both functions are symmetric around the $a$ axis since $x$ and $y$ electrodes have opposite electric fields. Figure 5.7 shows the first stability region.

For the Quattro II mass spectrometer quadrupoles, $a$ and $q$ can be calculated and a similar diagram can be obtained, this time as a function of the DC and RF voltages for different ion $\mathrm{m} / \mathrm{z}$. Figure 5.8 shows the stability region in the DC-RF plane for two ion species, ${ }^{138} \mathrm{Ba}^{+}$and ${ }^{138} \mathrm{Ba}^{++}$. Coincidentally, the value of the $R F$ voltage in volts is very similar to the $\mathrm{m} / \mathrm{z}$ value that has a stable trajectory. A mass scan gradually increases the RF and the $\mathrm{DC}$ voltages and goes through successive stability regions. Depending on the slope of the scan, a lower or higher $\mathrm{m} / \mathrm{z}$ resolution can be obtained. The two straight lines shown in Figure 5.8 represent 


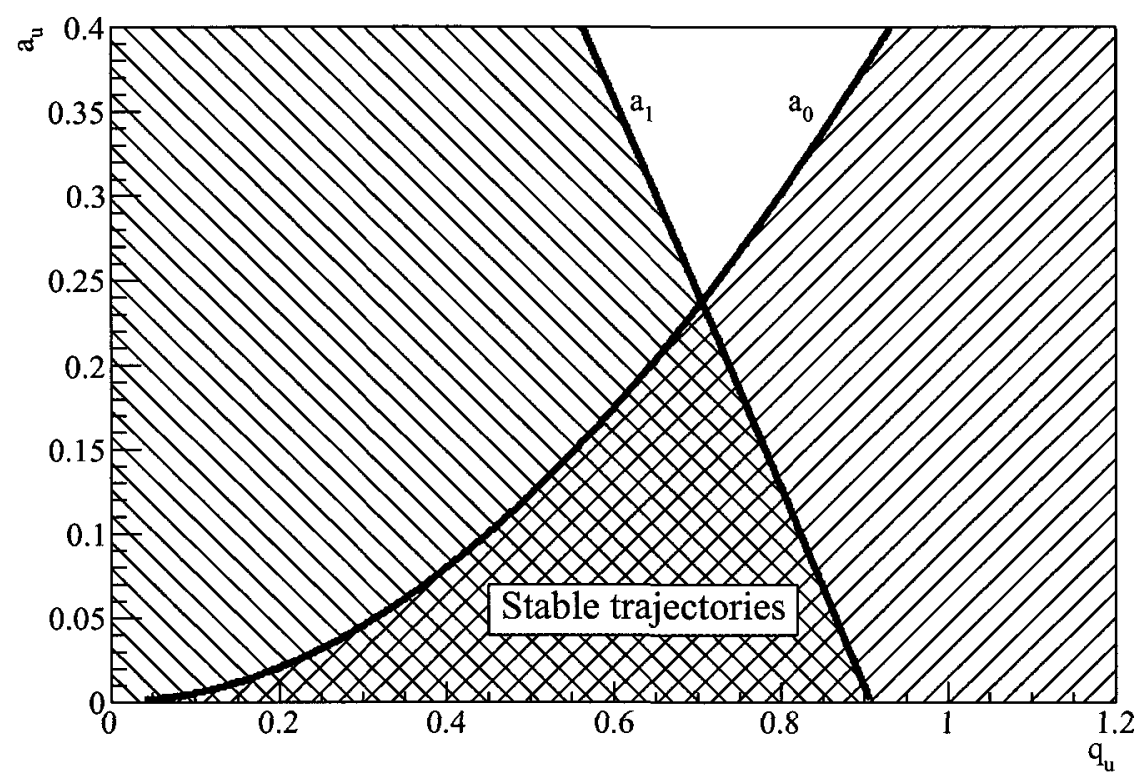

Figure 5.7: Mathieu function stability regions in the $a-q$ plane.

two arbitrary $\mathrm{DC} / \mathrm{RF}$ scan ratios. The ion signal yield can be found where the line passes through a stability region. A higher $\mathrm{DC} / \mathrm{RF}$ slope will result in a better resolution, as shown by the broken line. For a constant DC/RF ratio, the width of a mass peak will be wider at higher masses. A more constant resolution can be achieved by setting the intercept at the origin lower than 0. Modern instruments can even vary the $\mathrm{DC} / \mathrm{RF}$ ratio during the scan, resulting in a constant mass resolution across the whole mass range.

The Quattro II instrument allows setting two parameters to modify the resolution, LM Res and HM Res (low mass and high mass resolution). These are usually set between 5 and 25 , but no indication is given about the way the scan is performed. In order to try to understand how these parameters work, data has been collected varying both resolution parameters. The source used was the same barium acetate $1 \mathrm{mM}$ solution as described in the previous section. Figure $5.9 \mathrm{a}$ 

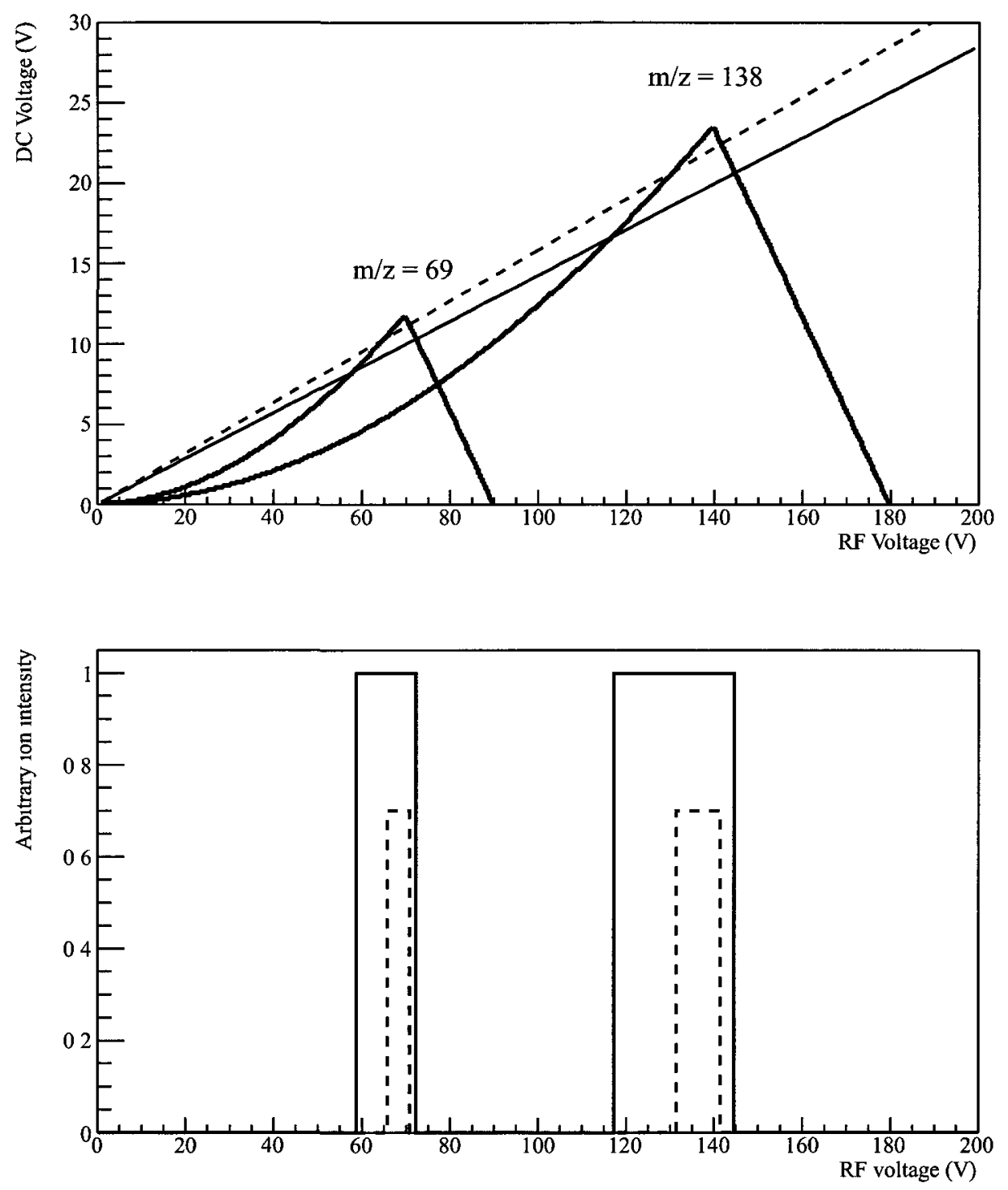

Figure 5.8: DC-RF stability regions for ${ }^{138} \mathrm{Ba}^{+}$and ${ }^{138} \mathrm{Ba}^{++}$of the Quattro II mass spectrometers. Two different $\mathrm{RF}$ to $\mathrm{DC}$ ratio mass scans give two different mass resolution spectra as indicated by the solid and the broken lines. The RF to DC ratios are arbitrary. 
shows the FWHM of the $\mathrm{Ba}^{++}$line for different LM Res and HM Res values. The best resolution seems to be around 0.15 Da. The same information is plotted in Figure 5.9b, but with the barium monoacetate ion peak at 197 Da.

Both peaks shows a linear decrease of the FWHM at a rate of $-0.05 \mathrm{Da} / \mathrm{LM}$ Res. Since the instrument can probe masses up to $4000 \mathrm{Da}$, both peaks at $\mathrm{m} / \mathrm{z}=69$ and $\mathrm{m} / \mathrm{z}=197$ are considered low masses, which explains similar behaviours with respect to the LM Res parameter. Is is also expected that the barium monoacetate peak would be more sensitive than the $\mathrm{Ba}^{++}$peak to the HM Res parameter. Each HM Res step increases the FWHM by a larger amount for barium acetate than for doubly charged barium. In addition, the FWHM is different for the barium monoacetate than for doubly charged barium at the same resolution parameters. This indicates that the mass spectrometer does not adjust the DC/RF ratio during a scan to get a constant mass resolution. Therefore, the LM Res parameter could control a constant bias of the DC voltage at the origin while HM Res would do a similar thing at the other end of the mass spectrum.

The peak intensity is very difficult to predict. The stability regions of the Mathieu function described are the product of many approximations. The most important one is the shape of the rods that does not create a perfect quadrupole field. The expansion of Equations 5.8a and 5.8b also includes some uncertainties. Finally, individual ions will have an initial velocity and position different from zero, which will lead to different trajectories. An ion that has the right mass might be on an unstable trajectory if the initial conditions are out of the ordinary. Instead of having a mass spectrum with sharp rectangles as shown in Figure 5.8, peaks are much smoother. Figures $5.10 \mathrm{a}$ and $5.10 \mathrm{~b}$ show the intensity of both the $\mathrm{Ba}^{++}$ and barium monoacetate ion peaks as a function of the LM Res and HM Res parameters. Again, the barium acetate peak depends much more strongly on the HM Res value than the doubly charge barium. 


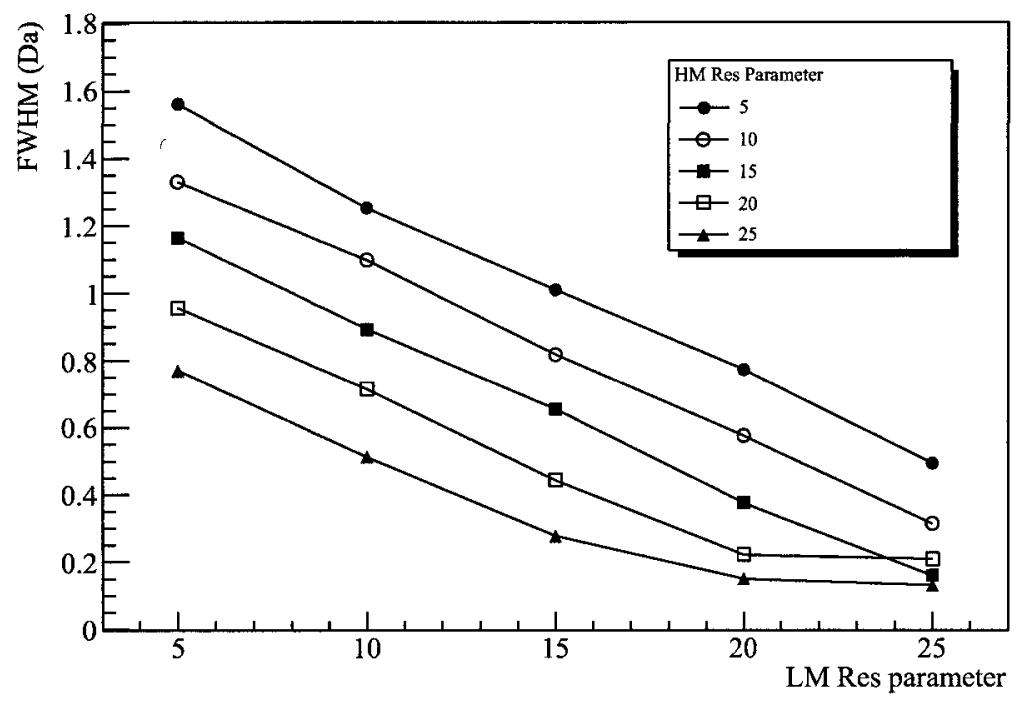

(a)

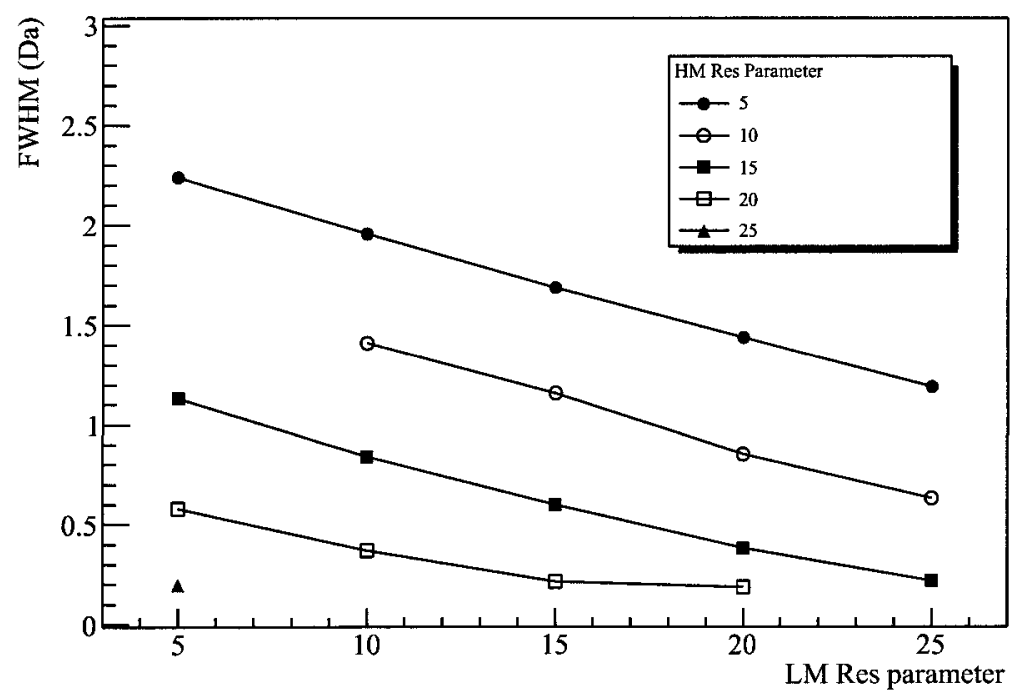

(b)

Figure 5.9: FWHM of the (a) $\mathrm{Ba}^{++}$and (b) the barium monoacetate ${ }^{+}$peaks for different LM Res and HM Res values. 


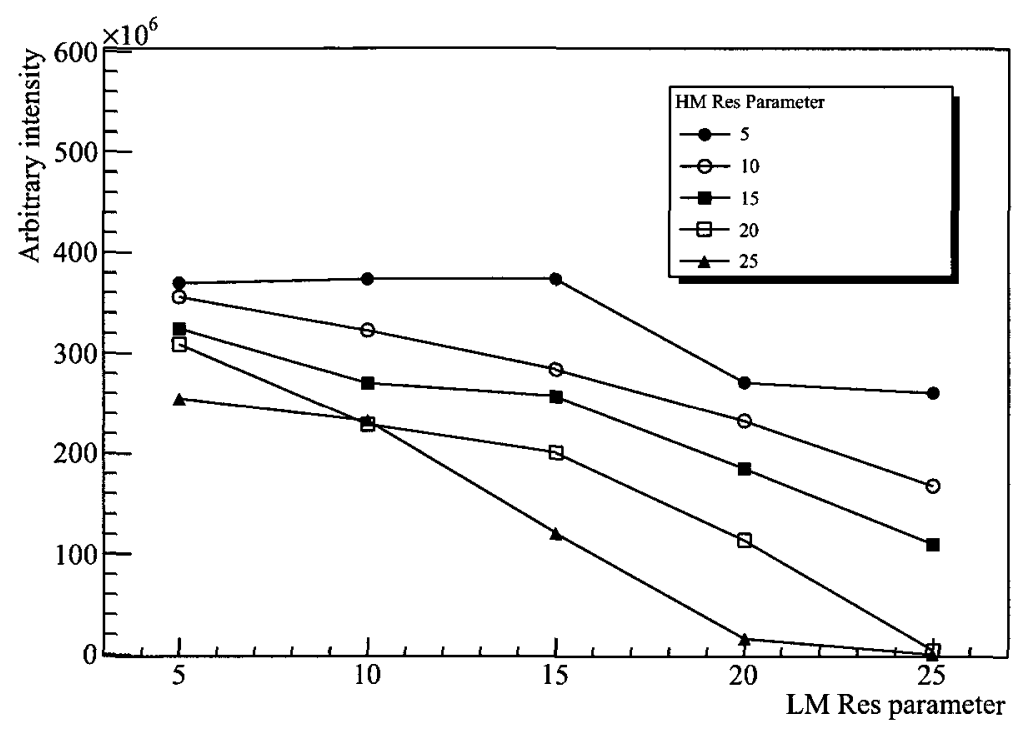

(a)

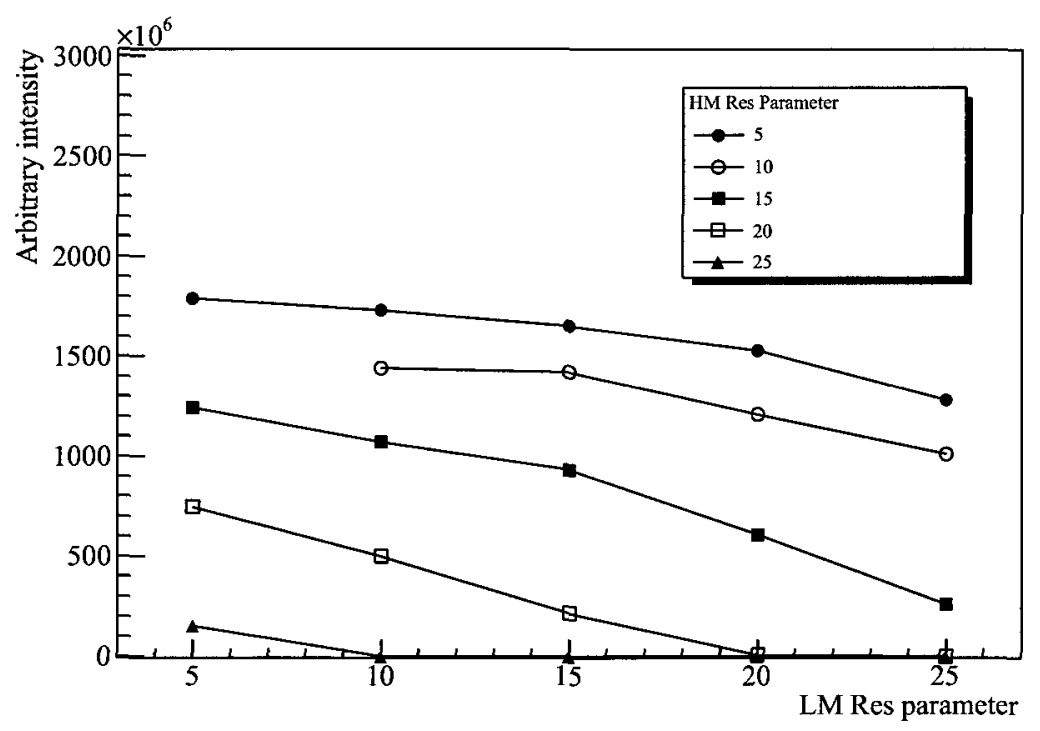

(b)

Figure 5.10: Intensity of the (a) $\mathrm{Ba}^{++}$and (b) barium monoacetate ${ }^{+}$peaks for different LM Res and HM Res values. 


\subsubsection{Collision Cell}

An important part of the mass spectrometer is the collision cell. It has two $2 \mathrm{~mm}$ diameter apertures to let ions enter and exit. It can be filled with gas, with a pressure from $10^{-4}$ to $10^{-2}$ mbar. It is about $16 \mathrm{~cm}$ long, so ions collide only a few times before leaving the cell. Ions are transported through the cell within the hexapole guide which should have an efficiency close to $100 \%$. The hexapole guide is not mass selective.

\subsubsection{Mass Spectrometer Operation Methods}

The mass spectrometer can be operated in many different modes; three of them were used for this study. The MS1 method activates the first mass spectrometer and disables the collision cell and the second mass spectrometer. It is the simplest way to measure a mass spectrum. The MS2 mode is the inverse. It disables the first mass spectrometer, but activates the collision cell and the second mass spectrometer. This mode can be used to monitor the effect of the collision cell but it is not very important since it does not control the ion type going in the collision cell. Finally, the most interesting mode is the Daughter mode. It uses the first mass spectrometer to filter out ions outside a mass window. Then, the remaining ions collide with the gas in the collision cell and the second mass spectrometer scans the mass range to find products created by the collisions. For example, if $\mathrm{m} / \mathrm{z}=197$ is selected to go through the collision cell filled with argon, it can collide with the argon atoms and fragment in smaller molecules. Figures 5.11a and 5.11b show this effect. The first one shows the Daughter spectrum with a collision voltage of $0 \mathrm{~V}$ while for the second one the collision voltage was increased to $100 \mathrm{~V}$. On the first one, $\mathrm{Ba}\left(\mathrm{CH}_{3} \mathrm{COO}\right)^{+}$at $\mathrm{m} / \mathrm{z}=197$ did not react with argon and it is the only product measured. At the higher collision energy, the molecule breaks up into fragments. $\mathrm{BaOH}^{+}$and $\mathrm{BaCH}_{3}^{+}$share a peak around $\mathrm{m} / \mathrm{z}=154$ and $\mathrm{Ba}^{+}$is the most intense peak at $m / z=138$. Some fragments are not measured, probably 
because they are neutral molecules or negative ions.

\subsubsection{Mass Spectrometer Calibration}

An accurate mass calibration of the instrument is useful to identify unknown peaks. After identifying known lines in a spectrum, the instrument fits a polynomial with a specified number of parameters to determine the scale of $\mathrm{m} / \mathrm{z}$. In the user manual, they suggest using a mix of $\mathrm{CsI}$ or $\mathrm{RbI}$ and $\mathrm{NaI}$ at low masses and horse myoglobin for masses above $1000 \mathrm{Da}$. After a few unsuccessful trials using $\mathrm{Cs}^{+}$and $\mathrm{Na}^{+}$, because the $\mathrm{Na}^{+}$line was not visible, it was decided to use barium acetate as the calibration source. Two masses were picked for the calibration, ${ }^{138} \mathrm{Ba}^{++}$and ${ }^{138} \mathrm{Ba}\left(\mathrm{CH}_{3} \mathrm{COO}\right)^{+}$. Both are very easy to identify because of the isotope content of barium that forms a very recognizable pattern as shown in Figure 5.4b. Then, a first order polynomial was fitted and calibration parameters were applied. Table 5.3 shows the uncalibrated measured mass, the expected mass and the difference between them. Once the calibration was applied to the data, the same exercise was performed on a different run to check the validity of the calibration. Table 5.4 shows the mass measurement of the ${ }^{138} \mathrm{Ba}^{+}$line, which was not used during the calibration and the ${ }^{133} \mathrm{Cs}^{+}$peak from a CsI solution. The accuracy of the mass measurement is better than $0.05 \%$. The stability of the instrument is also outstanding. The location of the $\mathrm{Ba}^{++}$peak was measured by the first mass spectrometer for different runs. Out of 54 runs taken on two different days, the variation of the peak was $0.014 \mathrm{Da}$, a tiny $0.02 \%$.

\subsection{Charge Transfer Probability}

The mass spectrometer can be programmed to carry out a set of automated measurements. For the following data, all three methods described in Section 5.1.4 were used. One second duration scans were recorded, alternating between MS1, 


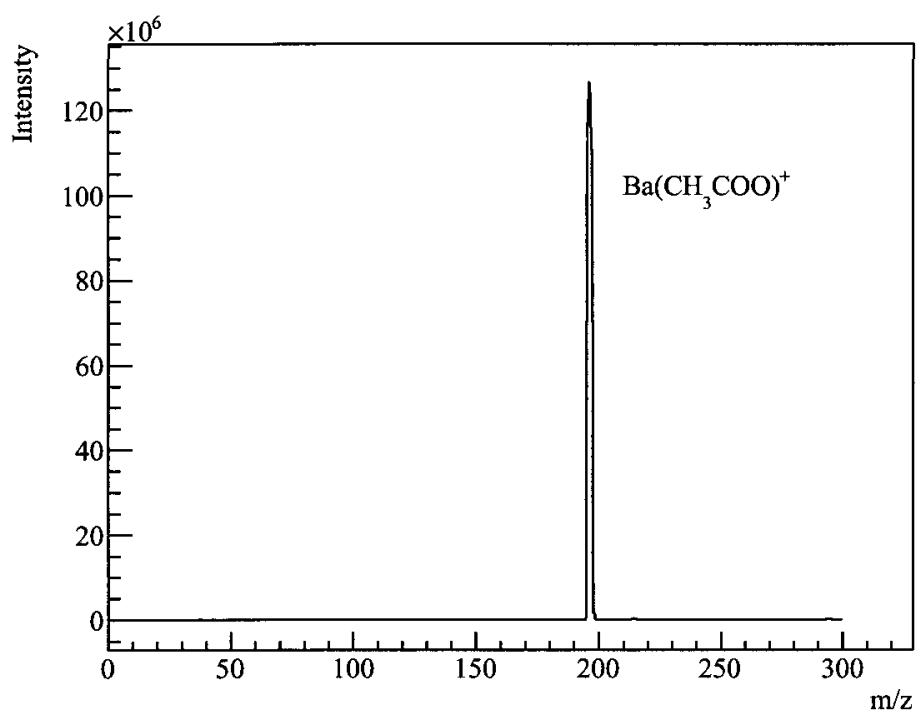

(a)

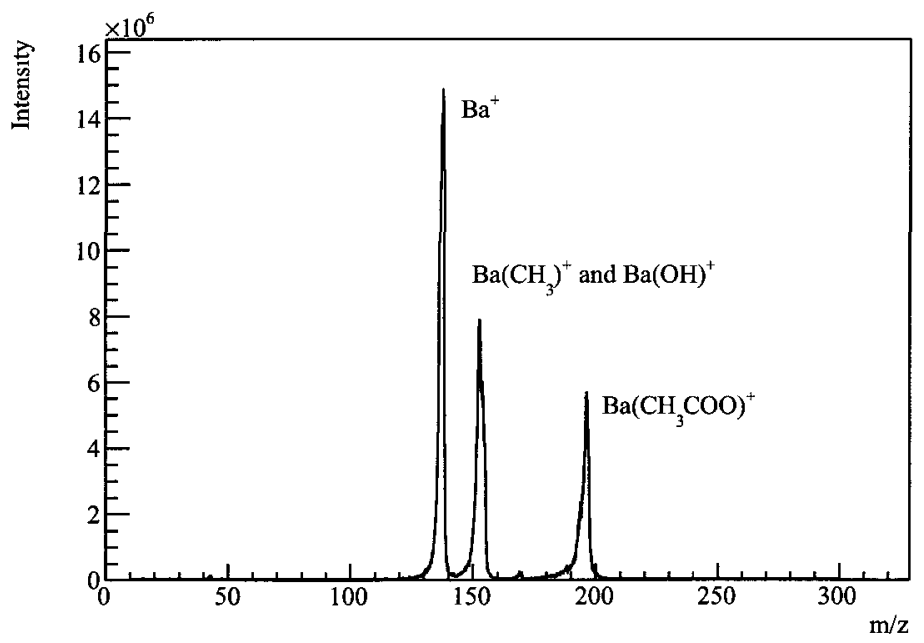

(b)

Figure 5.11: Fragmentation of barium mono-acetate at $0 \mathrm{~V}$ (a) and $100 \mathrm{~V}$ (b) in $3 \times 10^{-4}$ mbar of argon. The spectra were recorded with the Daughter method filtering a mass of $\mathrm{m} / \mathrm{z}=197$. 


\begin{tabular}{|c|c|c|c|}
\hline Molecule & Uncalibrated $\mathrm{m} / \mathrm{z}$ & Expected $\mathrm{m} / \mathrm{z}$ & Difference \\
\hline \hline${ }^{138} \mathrm{Ba}^{++}$ & 69.17 & 68.95 & 0.22 \\
\hline${ }^{138} \mathrm{Ba}\left(\mathrm{CH}_{3} \mathrm{COO}\right)^{+}$ & 197.22 & 196.92 & 0.30 \\
\hline
\end{tabular}

Table 5.3: Peaks used for the calibration of the mass spectrometer with the measured $\mathrm{m} / \mathrm{z}$ in the first mass spectrometer, the expected value and the difference between the two.

\begin{tabular}{|c|c|c|c|}
\hline Molecule & Calibrated m/z & Expected $\mathrm{m} / \mathrm{z}$ & Difference \\
\hline \hline${ }^{133} \mathrm{Cs}^{+}$ & 132.85 & 132.91 & 0.06 \\
\hline${ }^{138} \mathrm{Ba}^{+}$ & 137.91 & 137.87 & 0.04 \\
\hline
\end{tabular}

Table 5.4: List of peaks measured after the instrument calibration in the first mass spectrometer. Measured and expected values are listed with the differences between them.

MS2 and Daughter method, for a total of three minutes for each run.

The most important requirement for the barium tagging procedure is a high probability that a $\mathrm{Ba}^{++}$ion captures an electron. TEA was selected as the electron donor gas because of its low ionization potential of $7.5 \mathrm{eV}$. With $\mathrm{Ba}^{++}$having an electron affinity of $10 \mathrm{eV}$, the transfer should be rapid. Once converted into a $\mathrm{Ba}^{+}$ ion, further electron transfer would be unlikely since its electron affinity is reduced to $5.71 \mathrm{eV}$. TEA is used in TPCs as a quencher and can also act as a wavelength shifter. It was available, previously purchased for some prototype work for the EXO TPC. TEA is a liquid and its vapour pressure at room temperature is about 80 mbar [66], more than sufficient for the collision cell study.

The charge transfer measurement was performed using the Daughter method selecting $\mathrm{m} / \mathrm{z}=69$. This ensures that only $\mathrm{Ba}^{++}$ions enter the collision cell. The appearance of $\mathrm{Ba}^{+}$peak in the second mass spectrometer would show the transfer of an electron from a TEA molecule to a $\mathrm{Ba}^{++}$ion. The presence of $\mathrm{TEA}^{+}$is also 
expected. Figure 5.12 shows examples of the mass spectrum using the Daughter method on $\mathrm{m} / \mathrm{z}=69$, different TEA pressures and a collision voltage of $250 \mathrm{~V}$. Note that at $5.5 \times 10^{-3}$ mbar of TEA, there is no sign of $\mathrm{Ba}^{++}$.

Assuming that the response is constant across the mass range, the ratio between $\mathrm{Ba}^{+}$and the sum of $\mathrm{Ba}^{+}$and $\mathrm{Ba}^{++}$gives the conversion probability. Figure 5.13 shows this conversion probability as a function of TEA pressure and collision energy. The data shows a clear dependence on the pressure. As the pressure reaches $10^{-3}$ mbar, almost all barium ions are converted into $\mathrm{Ba}^{+}$. The conversion probability also depends on the collision voltage. A lower voltage means a higher conversion probability.

It seems that with only $10^{-4}$ mbar of TEA, the conversion probability $(P)$ reaches 65 to $85 \%$. The cross-section $(\sigma)$ of the reaction is calculated with

$$
\sigma=\frac{P}{n l}
$$

where $n$ is the TEA density and $l$ the length of the path in the gas. Assuming a TEA density of $2.4 \times 10^{18} \mathrm{~m}^{-3}$ within the $16 \mathrm{~cm}$ collision cell, the cross-section for the reaction is between $1.69 \times 10^{-18} \mathrm{~m}^{2}$ and $2.21 \times 10^{-18} \mathrm{~m}^{2}$. The measurement comes with the caveat that the pressure gauge of the collision cell is a Pirani gauge which depends on the gas type and no calibration for TEA was found. In addition, the trajectory of the ion in the cell is helicoidal, not straight. The $16 \mathrm{~cm}$ length is actually a lower limit and the cross-section of the reaction can be a bit lower.

Assuming that the TEA molecular radius is $3.2 \times 10^{-10} \mathrm{~m}$ [67] and the barium atomic radius is $2.2 \times 10^{-10} \mathrm{~m}$ [68], the hard sphere cross-section should be $9.2 \times$ $10^{-19} \mathrm{~m}^{2}$, similar to the reaction cross-section. The electron transfer seems to be occurring for low momentum transfer collisions since the TEA ${ }^{+}$mass peak does not suffer from a broadening as large as that for $\mathrm{Ba}^{+}$and $\mathrm{Ba}^{++}$as seen in Figure 5.12. Since the imparted energy is low, the electron transfer has to be made at large distances. So, it is not expected that the hard sphere cross-section would be a 

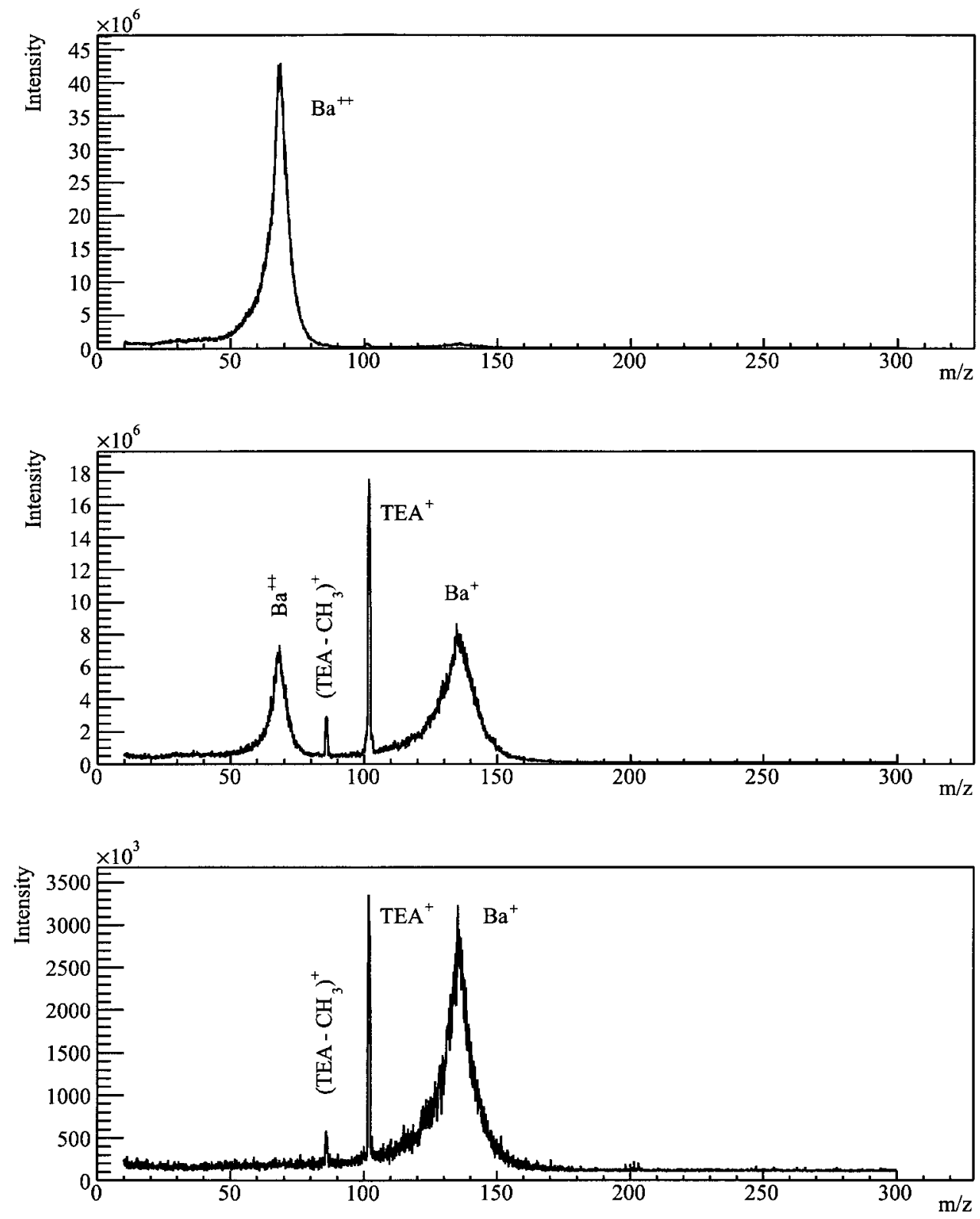

Figure 5.12: Examples of charge exchange spectra using TEA as the collision gas. The TEA pressure in the collision cell was less than $10^{-4}$ mbar for the upper plot, $3 \times 10^{-4}$ mbar for the central plot and $5.5 \times 10^{-3}$ mbar for the lower spectrum. The collision voltage was set at $250 \mathrm{~V}$. 


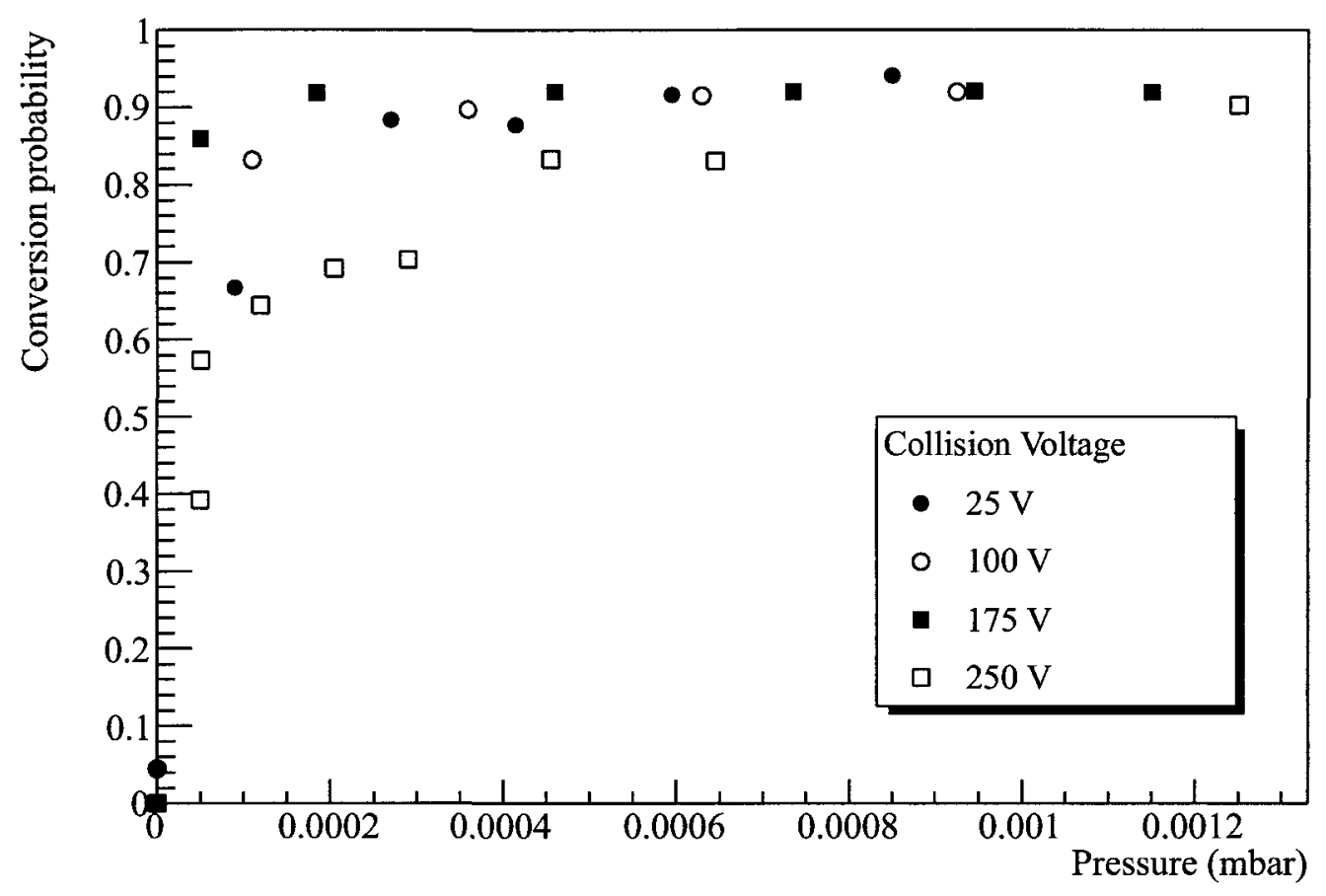

Figure 5.13: Conversion probability as a function of TEA pressure and collision energy.

good approximation of reality. Nevertheless, the transfer probability per collision is very likely to be close to $100 \%$. In fact, TEA was actually too effective. Even after removing the TEA source and pumping on the collision cell, conversion of the $\mathrm{Ba}^{++}$to $\mathrm{Ba}^{+}$was still occurring with the appearance of trace amounts of $\mathrm{TEA}^{+}$in the mass spectrum. Gas lines had to be purged and cleaned with isopropyl alcohol to remove TEA impurities.

From this study, two main questions are answered. $\mathrm{Ba}^{++}$can acquire an electron from TEA very easily and at pressures around $10^{-3}$ to $10^{-2}$ mbar the conversion is effectively $100 \%$. Furthermore, $\mathrm{Ba}^{++}$does not form molecules with TEA or any other contaminant present in the instrument. Nitrogen, water and methanol are introduced in the instrument from the source and the vacuum seals are sim- 
ple rubber o-rings, allowing even more contamination. In contrast, a $0 \nu \beta \beta$ decay experiment will be metal sealed and would have a part per billion contamination level. Therefore, $\mathrm{Ba}^{++}$to $\mathrm{Ba}^{+}$conversion for the laser fluorescence tagging in a gas phase xenon $0 \nu \beta \beta$ decay experiment should be very efficient. 


\section{Chapter 6}

\section{Conclusion}

The measurement of the neutrino mass is a very difficult task, but necessary to increase the knowledge of the Universe. This thesis has explained that observing the neutrinoless double beta decay is one of the most reasonable ways to experimentally measure it. It can also determine the nature of neutrinos and the underlying physics that allows neutrinos to acquire a mass. Even with an imprecise measurement of the angular correlation of the two electrons, valuable information can be extracted. Unfortunately, the sensitivity to new physics is too low for the physics model considered in this thesis. Nevertheless, this technique might be more sensitive to other models. Regardless of the potential for new physics, electron track reconstruction is a clear advantage to reduce the number of background events.

This thesis also included the description of two EXO prototypes that use xenon as the source of the decay. The liquid phase TPC (EXO-200) is in its commissioning phase. The gas phase TPC (XEP) is still under construction and will be used as a proof of principle experiment. No $0 \nu \beta \beta$ decay events will be observed with it. It has been shown, using simulations, that a meter size TPC would contain most of the $1 \mathrm{MeV}$ electrons if they are generated in the centre of the detector. In addition, adding a lighter gas like neon or argon would smooth the track shapes and reduce the number of high energy gammas. 
It has also been discussed that LIF is a very efficient technique for barium tagging in order to reduce the number of background events in the EXO experiment. Two barium ion sources were built to investigate tagging in gas. Unfortunately, the tagging in high pressure gas was not successful, although it was not determined if it is impossible. Repetitive failure of instrumentation and the development of new ideas to increase the efficiency of performing the tagging diverted the efforts to a different project. Nevertheless, some basic measurement of the barium ion spectroscopy were performed and valuable knowledge was acquired.

It has been determined during the study that extracting the ion from the high pressure xenon volume into an ion trap should be easier than performing the tagging in the xenon volume. Outside the TPC, the conversion of the doubly charged ion to the singly charged barium ion could be done without worrying about the contamination of the xenon volume by the electron donor gas. The main achievement of this thesis was to show that doubly charged barium ions can acquire an electron with nearly $100 \%$ efficiency when colliding with a TEA molecule. It has been demonstrated that barium ions do not form molecules with TEA. In fact, even in the presence of nitrogen and water vapour, doubly charged barium ion survives. Finally, it was shown that an electrospray source was capable of producing doubly charged barium ions.

Other research and development would be useful and will be included in future studies. For example, the measurement of the charge exchange using other gases, like xenon, could be even more appropriate for barium tagging in the context of the EXO experiment. Also, with a small modification of the electrospray source, one could measure the drift velocity of doubly charged barium in xenon at different pressures. It may also be possible to replace the electrospray source by a single barium ion source to proceed to an efficiency measurement. The mass spectrometer is a wonderful instrument, it already answered many questions about ion chemistry and should answer more in the near future.

In light of the results described in this thesis, a complete tagging procedure 
is likely to succeed, although the estimation of its efficiency is not possible yet. The current design of a barium tagging apparatus adapted to the XEP experiment would work as follow. When an ionizing event would be detected, a basic analysis of the track would provide the likelihood of the event to be a double beta decay event. It would also estimate the location of the barium daughter of the reaction, which would allow to initiate the extraction procedure through a small aperture by adjusting the electric field in the TPC. On the other side of the aperture, the xenon gas that exited the TPC would be pumped out of the tagging apparatus, but the barium ion would be kept in the centre of an hexapole ion trap. At the same time, a electron donor buffer gas like TEA would convert the doubly charge ion into a singly charged one, allowing the laser to initiate LIF of the barium ion while the ion would still be confined in the same trap. 


\section{Appendix A}

\section{Analysis of the hep neutrino flux with the Sudbury Neutrino Observatory}

This appendix describes the work performed at the beginning of my Ph.D. program. It involved skills previously acquired handling the SNO data and its simulation packages. The purpose of it was to investigate the hep neutrino flux, an analysis never performed by the SNO collaboration and it resulted in a published paper [69].

Understanding of the physics of the Sun has been expanded by the study of solar neutrinos which are constantly produced by the Sun. For more than three decades, scientists were puzzled by the discrepancy between the calculated and the measured neutrinos flux. Only a third of electron neutrinos produced by the Sun reach the Earth. SNO has been designed to solve the solar neutrino problem by measuring both the electron neutrino flux and the total active neutrino flux [4]. After a few years of data taking SNO had solved the problem by showing that neutrinos oscillate between all three flavours. Therefore, neutrinos produced by the Sun in the electron flavour could change into muon and tau flavours, which 
were not detected by previous experiments.

The SNO energy threshold of $5 \mathrm{MeV}$ only allows the detection of neutrinos produced by the ${ }^{8} \mathrm{~B}$ and the hep reactions (see Table A.1). The ${ }^{8} \mathrm{~B}$ process dominates over the hep by a factor close to 500, but part of the hep spectrum extends beyond the ${ }^{8} \mathrm{~B}$ energy endpoint as depicted in Figure A.1. Although the observation of hep neutrinos is challenging, there are advantages to a hep analysis. The calculation of the hep flux is very uncertain due to the unknown matrix elements involved in the hep reaction. John Bahcall wrote "I have been unable to find a reliable way of estimating an uncertainty in this production cross section, since the reaction is forbidden and the calculated value involves strong cancellation between terms from different sources" [70]. The current uncertainty is estimated to be $15.1 \%$ [71] and a hep flux measurement would be the only reasonable way to measure the cross section of the hep reaction. Also, since hep neutrinos are produced further away from the centre of the Sun than the ${ }^{8} \mathrm{~B}$ neutrinos, their path through the Sun is different. By measuring the difference in the survival probabilities of ${ }^{8} \mathrm{~B}$ and hep neutrinos (probability that $\nu_{e}$ stays a $\nu_{e}, P_{e e}$ ), it is possible to gain valuable information on the Sun temperature, composition and density profiles. Figure A.2 shows the radial distribution of the production of ${ }^{8} \mathrm{~B}$ and hep neutrinos in the Sun according to the Solar Model of Bahcall 2000 [72]. The production location of ${ }^{8} \mathrm{~B}$ neutrinos depends strongly on the temperature $\left(\Phi\left({ }^{8} \mathrm{~B}\right) \propto \mathrm{T}^{18}\right)$ explaining the central location of their origin. For hep neutrinos, they depend less strongly on the temperature $\left(\Phi(\right.$ hep $) \propto \mathrm{T}^{3}$ to $\left.{ }^{6}\right)$ and the ${ }^{3} \mathrm{He}$ density increases when going away from the centre, with a maximum or ${ }^{3} \mathrm{He}$ at $0.27 R_{\text {Sun }}$.

Two types of analysis were considered. If the number of events is large enough, one can fit the signal and background distributions. The second type of analysis is called a box analysis and it involves counting the number of events inside an energy window and comparing it with the expected number of background events. If the number of events is significantly higher than the background, one can extract a measurement, otherwise one calculates an upper limit. 


\begin{tabular}{|c|c|c|c|}
\hline Reaction & Description & $\begin{array}{c}\text { Calculated Flux } \\
\left(\times 10^{3} \mathrm{~cm}^{-2} \mathrm{~s}^{-1}\right)\end{array}$ & $\begin{array}{c}\text { Energy endpoint } \\
(\mathrm{MeV})\end{array}$ \\
\hline \hline${ }^{8} \mathrm{~B}$ & ${ }^{8} \mathrm{~B} \rightarrow{ }^{8} \mathrm{Be}+\mathrm{e}^{+}+\nu_{e}$ & $4500 \pm 720$ & 15 \\
\hline hep & ${ }^{3} \mathrm{He}+\mathrm{p} \rightarrow{ }^{4} \mathrm{He}+\mathrm{e}^{+}+\nu_{e}$ & $8.3 \pm 1.2$ & 18.8 \\
\hline
\end{tabular}

Table A.1: The two reactions producing neutrinos that are detected by SNO with a description of their reaction, flux and energy endpoint.

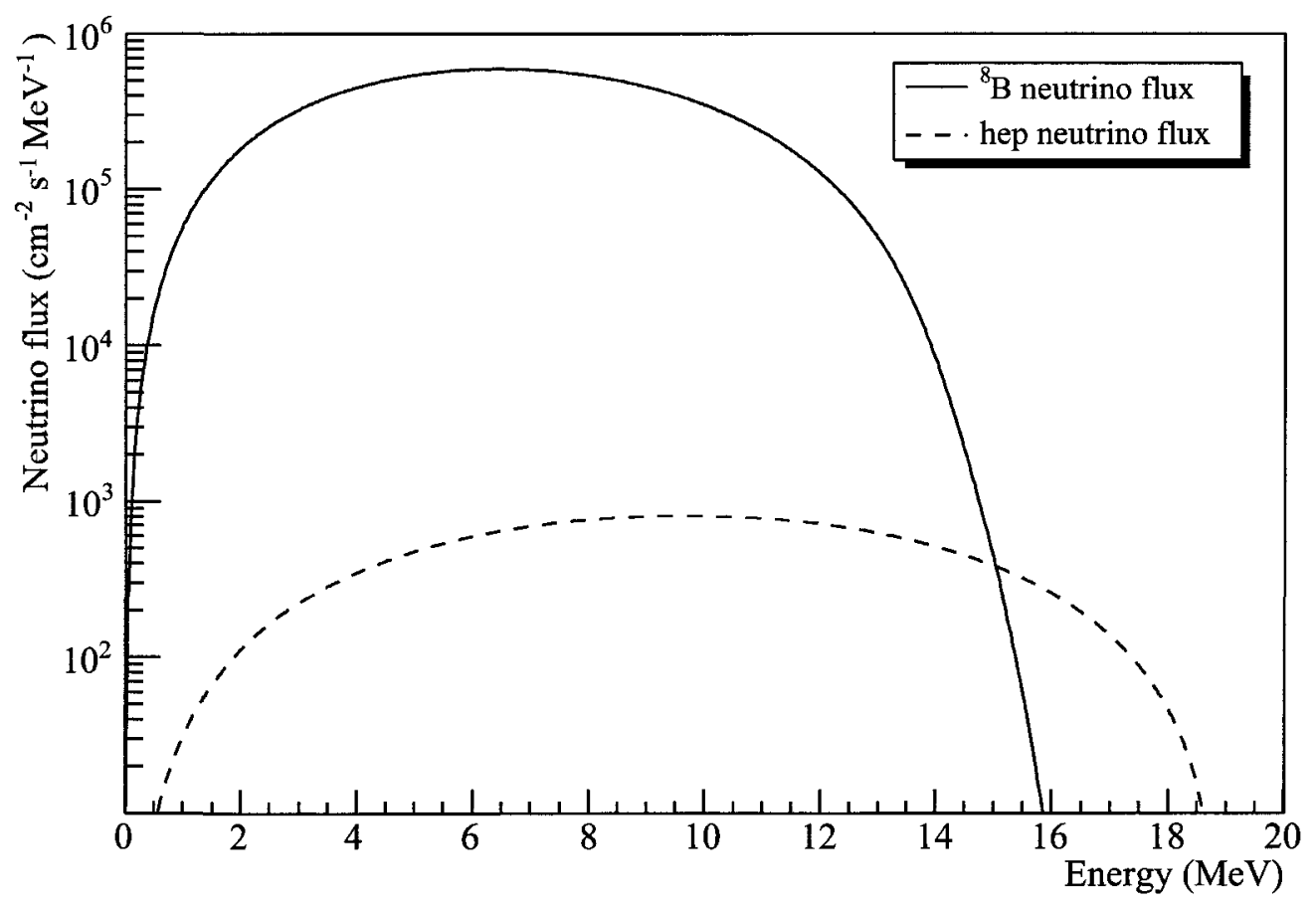

Figure A.1: ${ }^{8} \mathrm{~B}$ and hep neutrino fluxes at the surface of the Earth. 


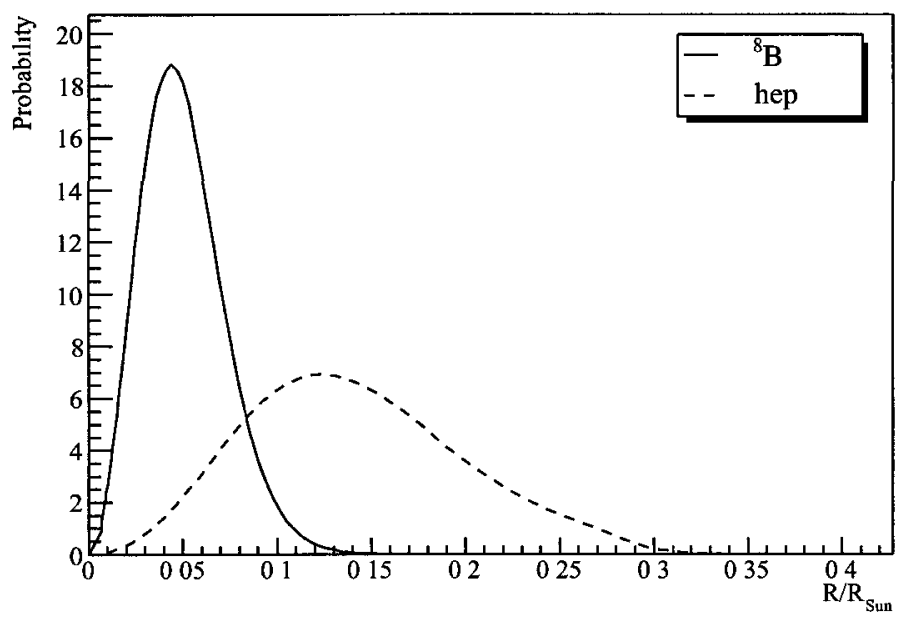

Figure A.2: Radial distribution of the production of ${ }^{8} \mathrm{~B}$ and hep neutrinos in the Sun.

After the first data taking phase of $\mathrm{SNO}\left(\mathrm{D}_{2} \mathrm{O}\right.$ phase), the collaboration decided to analyze the data to measure the hep neutrino flux. The box analysis which counts the number of events within an energy window was chosen. The details of the technique and the results are described in the SNO hep paper [69]. The data used for this analysis was the entire first phase of SNO data taking, which contains 306.4 live days or an exposure of 0.65 ktons yr.

The energy range selected, based on a Monte Carlo sensitivity study, was from $14.3 \mathrm{MeV}$ to $20 \mathrm{MeV}$. Figure A.3 shows the expected number of events and backgrounds as a function of energy in the data. Below the hep neutrino window, the main background is the ${ }^{8} \mathrm{~B}$ neutrinos and above $20 \mathrm{MeV}$, atmospheric neutrinos dominate the dataset. 


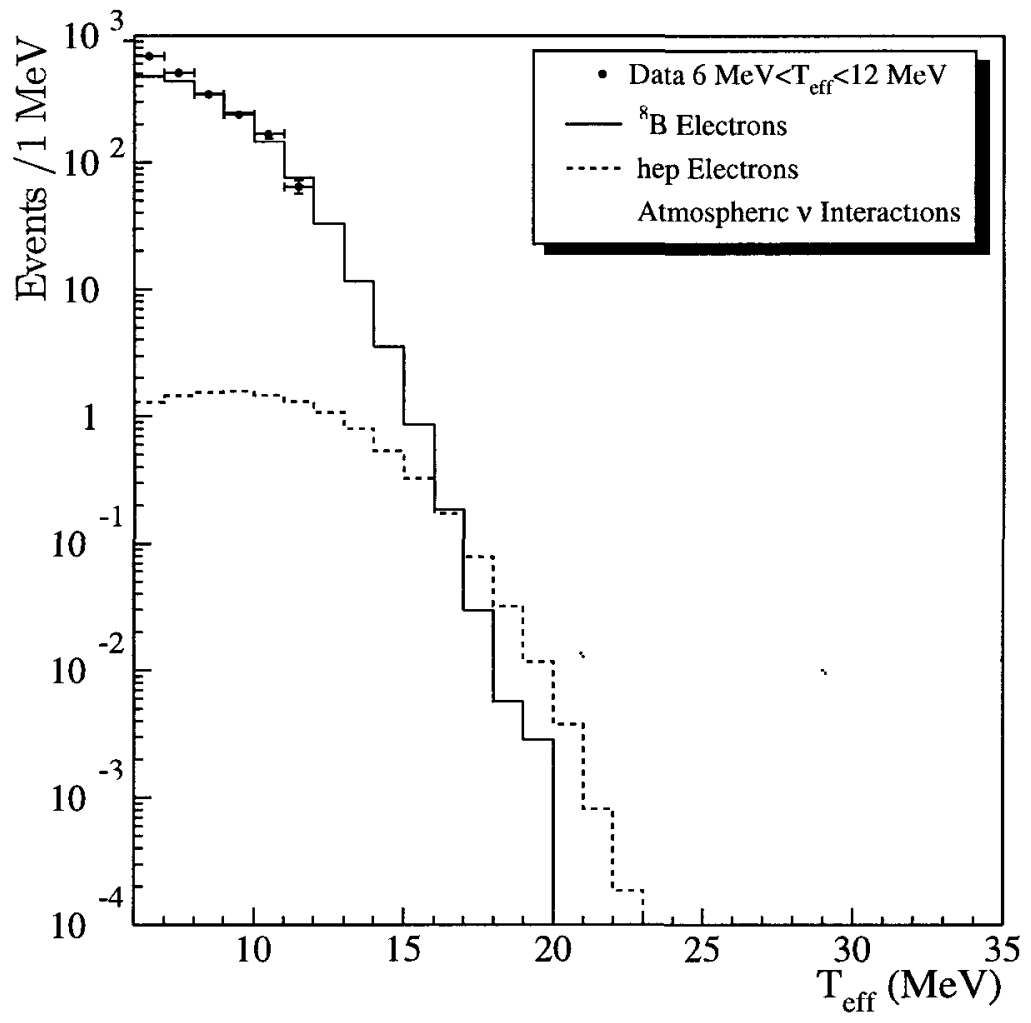

Figure A.3: Simulated spectra from different components of the signal and background for a hep neutrino study with the SNO data. The number of events has been calibrated to fit the data between 6 and $12 \mathrm{MeV}$, shown as reference only. 


\section{A.1 Systematic uncertainties}

\section{A.1.1 Energy resolution and energy scale}

Systematic errors in determining the hep flux are dominated by the energy scale and energy resolution of the SNO detector. The energy calibration was mainly performed with a ${ }^{16} \mathrm{~N}$ source, producing $6.13 \mathrm{MeV} \gamma$ rays. Extrapolating the calibration to 15 or $20 \mathrm{MeV}$ is a major source of uncertainty in the energy resolution, but fortunately a second $\gamma$ ray source was used. A pT source (tritium absorbing a proton) producing $19.8 \mathrm{MeV} \gamma$ was used to probe the high energy region and reduce the uncertainty related to the energy scale. Finally, a third energy scale measurement was made using Michel electrons (electrons from the decay of a muon stopping in the detector). Even with these three techniques, the major systematic uncertainties in the hep flux measurement are the energy resolution and the energy scale. A miscalibration of the energy scale of the detector would result in an incorrect estimation of the number of ${ }^{8} \mathrm{~B}$ events and also, at a lower level, the number of expected hep events.

\section{A.1.2 Oscillation parameters}

Another uncertainty in the number of events expected comes from the uncertainty in the two oscillation parameters $\Delta \mathrm{m}_{12}^{2}$ and $\tan ^{2} \theta_{12}$. They each influence oscillations differently for ${ }^{8} \mathrm{~B}$ and hep neutrinos. Taking two different points in the allowed region of the oscillation plane will give two different survival probability spectra. Therefore, sampling the allowed region in the oscillation plane will give different survival probabilities, and consequently give different fluxes for each neutrino flavour. Figure A.4 shows 7197 sample points of the Large Mixing Angle region of the neutrino oscillation plane according to the allowed region from a global solar neutrino analysis [69]. For each sampled point, a survival probability for the ${ }^{8} \mathrm{~B}$ and hep neutrinos was extracted and is shown in Figures A.5. 


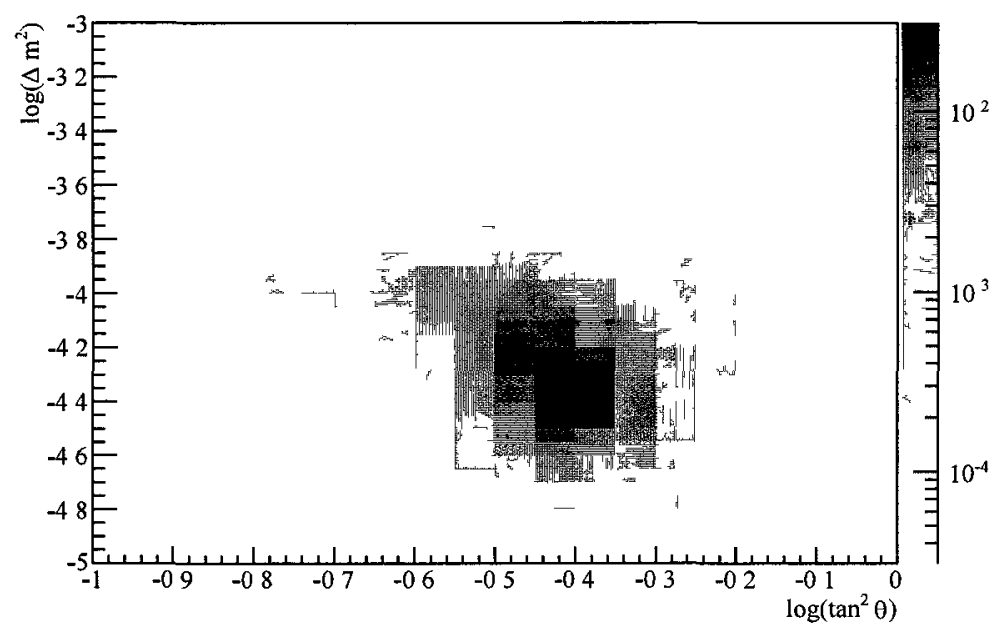

Figure A.4: Oscillation parameter sampling used to extract survival probabilities shown in Figure A.5.

The same analysis can be carried for ${ }^{8} \mathrm{~B}$ neutrinos and the width of the survival probability density plot can be measured for both hep and ${ }^{8} \mathrm{~B}$ neutrinos, and is shown in Figure A.6. In the hep window, the RMS of survival probabilities are of the order of $5 \%$ which has to be taken into account in the hep and background event estimation.

\section{A.1.3 Neutrino cross section}

In previous SNO analyses many parameters were integrated over the ${ }^{8} \mathrm{~B}$ spectrum. For the hep analysis these parameters had to be deconvoluted and considered for the whole energy range since the ${ }^{8} \mathrm{~B}$ spectrum is very different from than of the hep spectrum. One such parameter is the charged current (CC) to electron scattering (ES) cross section ratio, which is essential in the conversion of the number of events observed to the total neutrino flux.

The CC cross section uncertainty comes from many sources. The first one comes from the ratio of the axial to vector charge of the nucleon $\left(\mathrm{g}_{A} / \mathrm{g}_{V}\right)$. The 


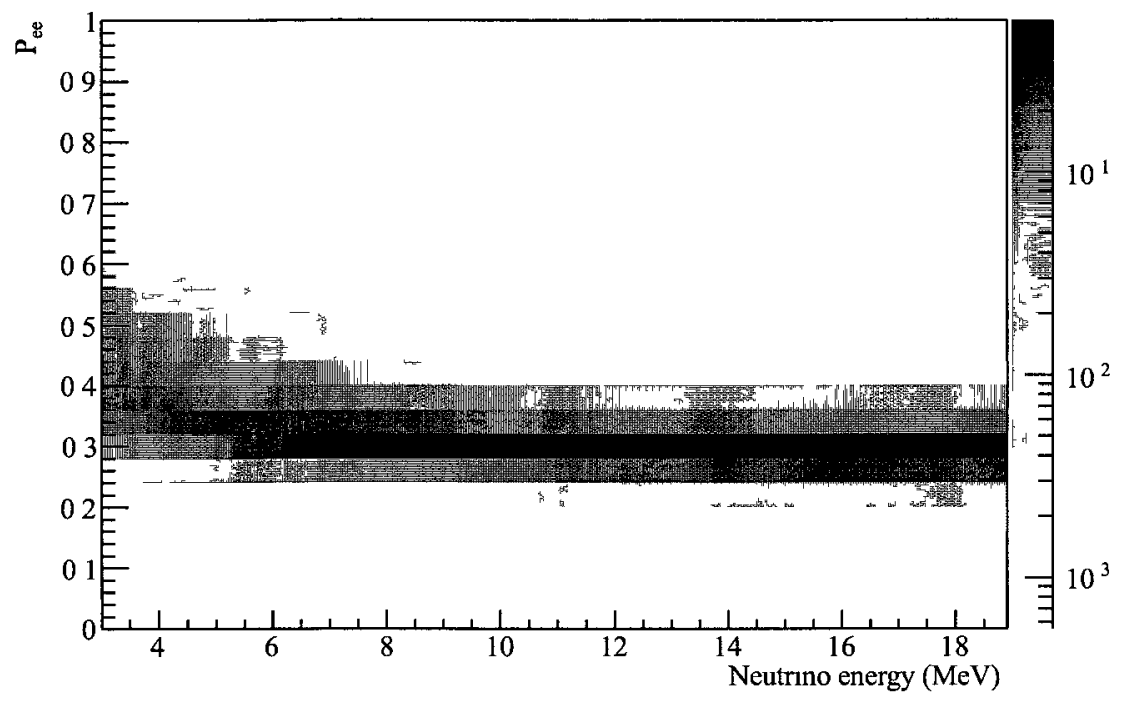

(a)

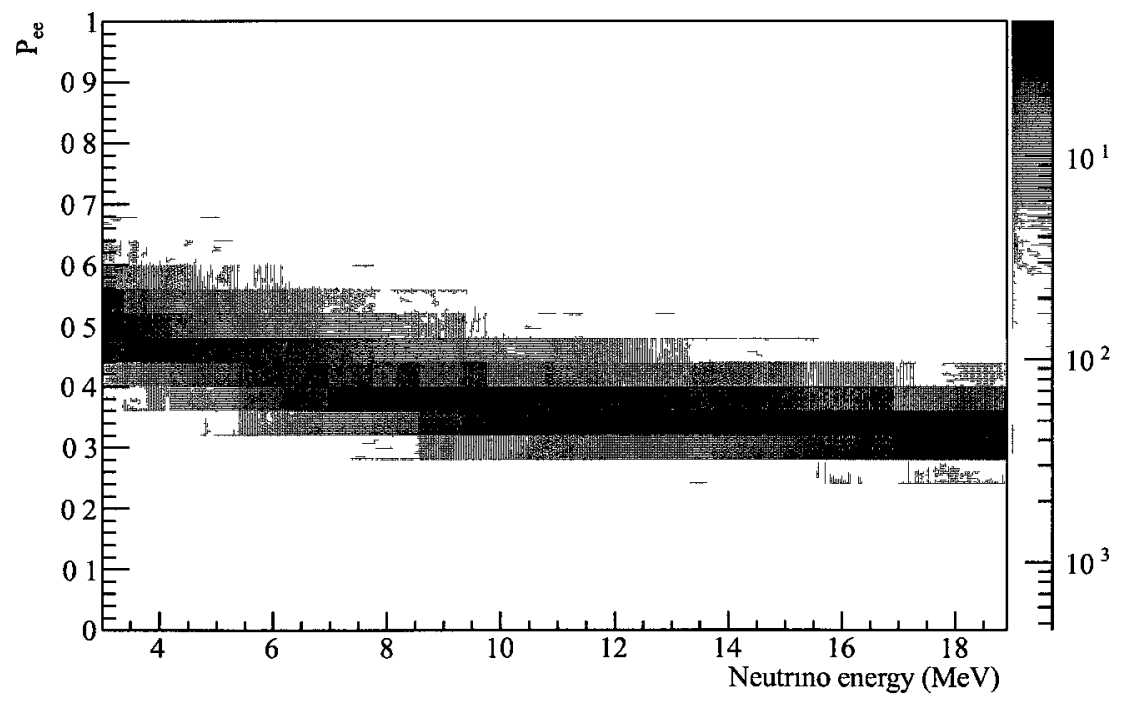

(b)

Figure A.5: Variation of the survival probability for ${ }^{8} \mathrm{~B}$ and hep neutrinos for 7197 oscillation parameter sets generated randomly according to the confidence region at the time of the publication of the SNO hep paper [69]. (a) is the survival probability for ${ }^{8} \mathrm{~B}$ and (b) is for hep neutrinos. 


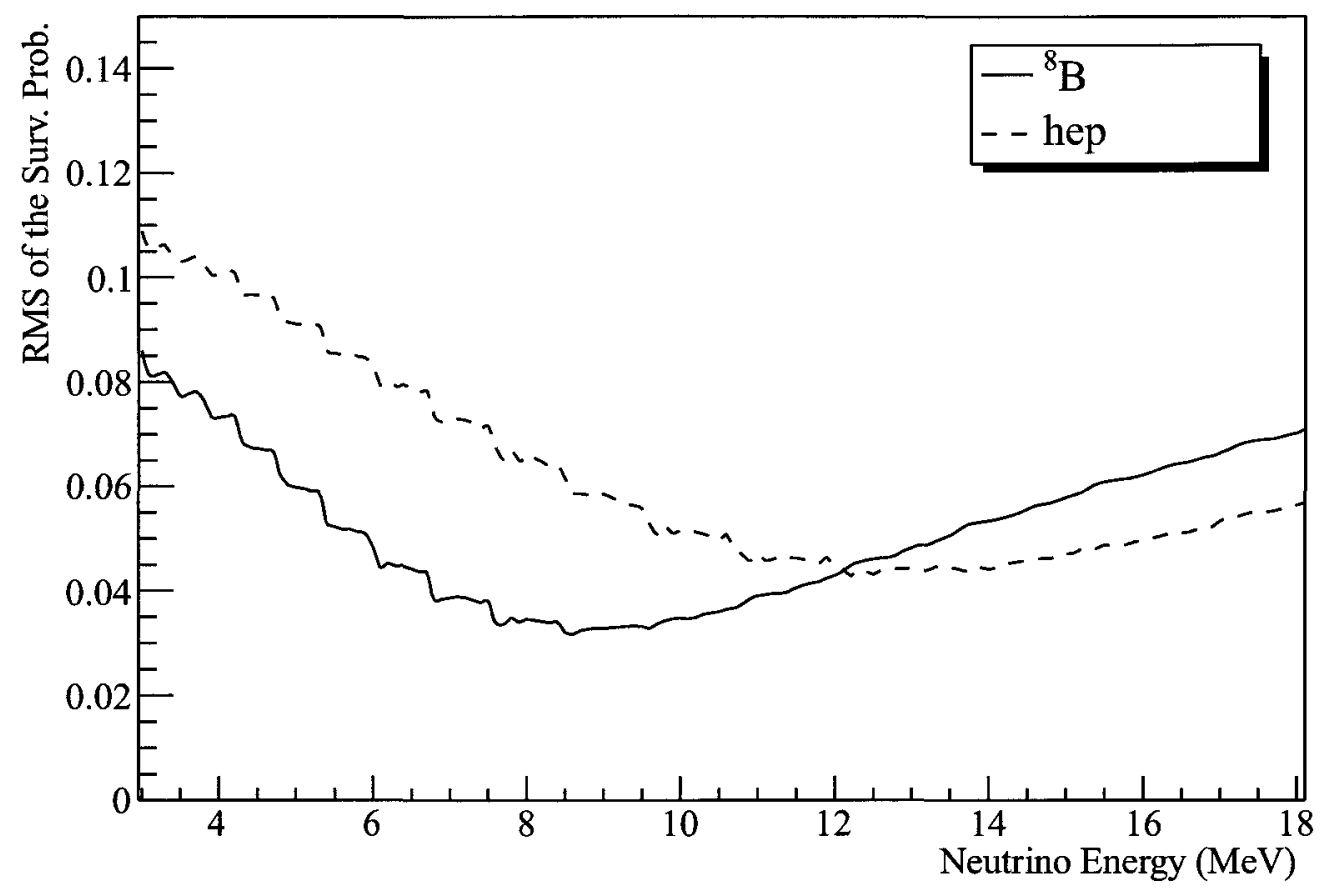

Figure A.6: Root mean square value of the survival probability distribution as a function of energy taken from Figure A.5, sampling the allowed region in the oscillation plane of Figure A.4 for both hep and ${ }^{8} \mathrm{~B}$ neutrinos. 
current value is $1.2670 \pm 0.0030$, which translates into a $0.5 \%$ uncertainty in the $\mathrm{CC}$ cross section calculation since the term is squared. Then, the uncertainty in the model itself can be accounted for by comparing the calculation of two different techniques. Here, NSA+ [73] and NSGK [74] are compared and in the hep window, the discrepancy was at most $0.6 \%$. Finally, an uncertainty is assigned to the handling of the radiative corrections by the SNO simulation package. First, the NSA+ model already includes the radiative corrections, but more precise ones are available (KVM [75]). Therefore, the SNO collaboration decided to subtract the radiative corrections from NSA+ and add the corrections from KVM which reduced the uncertainty to $0.3 \%$. Adding all uncertainties in quadrature gives a total uncertainty on the $\mathrm{CC}$ cross section of $0.9 \%$.

The ES cross section uncertainty was neglected in the first few SNO papers. For the hep analysis, a very conservative $0.5 \%$ uncertainty was assigned for the whole spectrum from which $0.1 \%$ comes from the theory. The main part of the uncertainty comes from the way radiative corrections are handled in the Monte Carlo simulation package of SNO.

\section{A.1.4 Other uncertainties}

Many other uncertainties have been included in the analysis. The vertex reconstruction of an event, the atmospheric neutrino flux, the fit on the ${ }^{8} \mathrm{~B}$ low energy normalization fit and the production of a $15.1 \mathrm{MeV} \gamma$ from ${ }^{12} \mathrm{C}$ decay, generated by neutrino interactions on oxygen are other uncertainties considered in the analysis. Details are included in the SNO hep paper [69], but contributions to the total uncertainty are small. 


\section{A.2 Results}

Results of the analysis are shown in Figure A.7, where the data and the expected background are shown as a function of energy. After integrating over the hep window, two events were observed while $3.1 \pm 0.60$ background and $0.99 \pm 0.09$ hep events were expected. The confidence interval analysis was performed with a modified Feldman-Cousins technique [76]. The technique calculates limits with a Monte Carlo analysis using the probability of observing the number of events from a signal, including systematic uncertainties described and their correlations. From the number of events counted, an upper limit result was appropriate. The analysis showed that at a $90 \%$ confidence level, the hep neutrino flux is lower than $2.3 \times 10^{4} \mathrm{~cm}^{-2} \mathrm{~s}^{-1}$. This number improves the previous limit by a factor of 6.5 , based on the Super-Kamiokande experiment, but it is still 3 times higher than the predicted hep flux of $8.3 \times 10^{3} \mathrm{~cm}^{-2} \mathrm{~s}^{-1}$. 


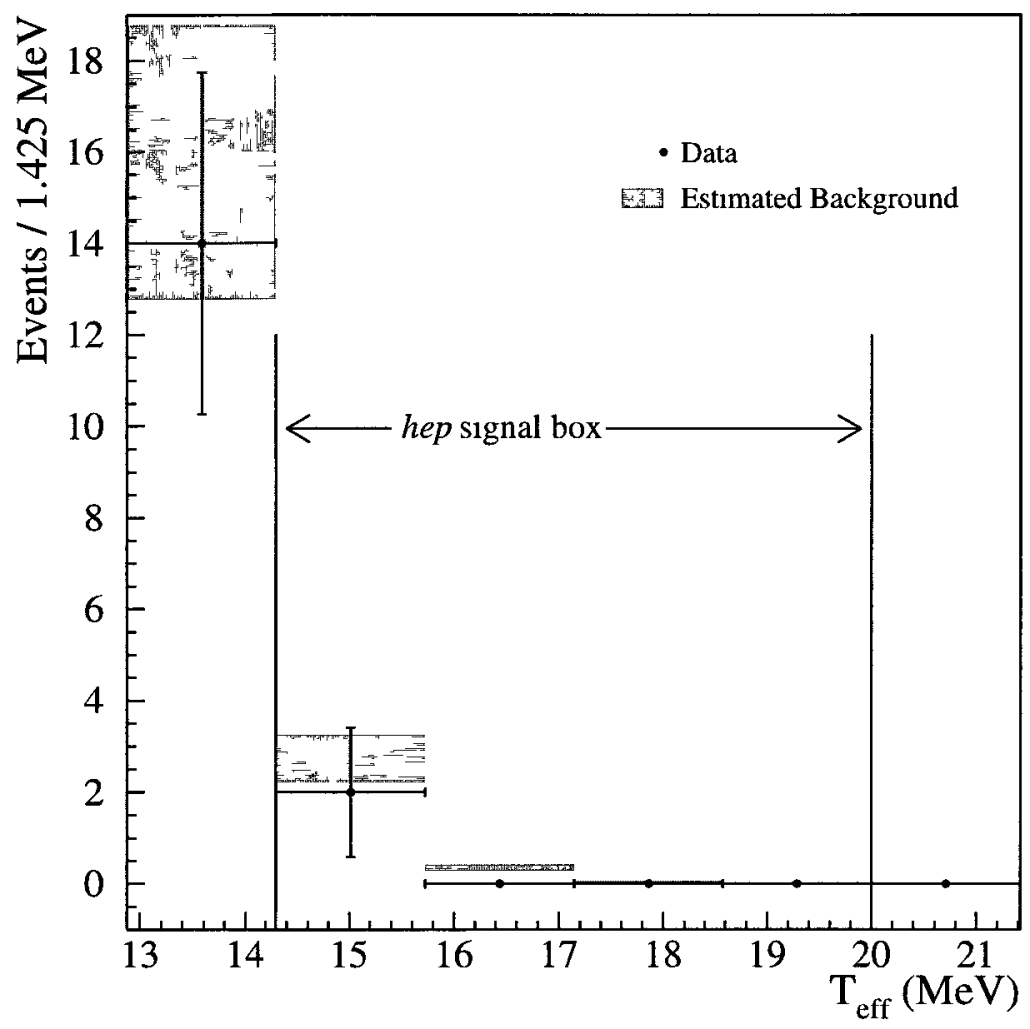

Figure A.7: Data from the $\mathrm{D}_{2} \mathrm{O}$ phase near the hep window with the estimated background and predicted hep signal as a function of energy. Uncertainty in the data and the estimated background are also shown. 


\section{References}

[1] C. Amsler, M. Doser, M. Antonelli, D.M. Asner, K.S. Babu, H. Baer, H.R. Band, R.M. Barnett, E. Bergren, J. Beringer, G. Bernardi, W. Bertl, H. Bichsel, O. Biebel, P. Bloch, E. Blucher, S. Blusk, R.N. Cahn, M. Carena, C. Caso, A. Ceccucci, D. Chakraborty, M.-C. Chen, R.S. Chivukula, G. Cowan, O. Dahl, G. D'Ambrosio, T. Damour, A. de Gouva, T. DeGrand, B. Dobrescu, M. Drees, D.A. Edwards, S. Eidelman, V.D. Elvira, J. Erler, V.V. Ezhela, J.L. Feng, W. Fetscher, B.D. Fields, B. Foster, T.K. Gaisser, L. Garren, H.-J. Gerber, G. Gerbier, T. Gherghetta, G.F. Giudice, M. Goodman, C. Grab, A.V. Gritsan, J.-F. Grivaz, D.E. Groom, M. Grnewald, A. Gurtu, T. Gutsche, H.E. Haber, K. Hagiwara, C. Hagmann, K.G. Hayes, J.J. Hernndez-Rey, K. Hikasa, I. Hinchliffe, A. Hcker, J. Huston, P. Igo-Kemenes, J.D. Jackson, K.F. Johnson, T. Junk, D. Karlen, B. Kayser, D. Kirkby, S.R. Klein, I.G. Knowles, C. Kolda, R.V. Kowalewski, P. Kreitz, B. Krusche, Yu.V. Kuyanov, Y. Kwon, O. Lahav, P. Langacker, A. Liddle, Z. Ligeti, C.-J. Lin, T.M. Liss, L. Littenberg, J.C. Liu, K.S. Lugovsky, S.B. Lugovsky, H. Mahlke, M.L. Mangano, T. Mannel, A.V. Manohar, W.J. Marciano, A.D. Martin, A. Masoni, D. Milstead, R. Miquel, K. Mnig, H. Murayama, K. Nakamura, M. Narain, P. Nason, S. Navas, P. Nevski, Y. Nir, K.A. Olive, L. Pape, C. Patrignani, J.A. Peacock, A. Piepke, G. Punzi, A. Quadt, S. Raby, G. Raffelt, B.N. Ratcliff, B. Renk, P. Richardson, S. Roesler, S. Rolli, A. Romaniouk, L.J. Rosenberg, J.L. Rosner, C.T. Sachrajda, Y. Sakai, S. Sarkar, F. Sauli, O. Schnei- 
der, D. Scott, W.G. Seligman, M.H. Shaevitz, T. Sjstrand, J.G. Smith, G.F. Smoot, S. Spanier, H. Spieler, A. Stahl, T. Stanev, S.L. Stone, T. Sumiyoshi, M. Tanabashi, J. Terning, M. Titov, N.P. Tkachenko, N.A. Trnqvist, D. Tovey, G.H. Trilling, T.G. Trippe, G. Valencia, K. van Bibber, M.G. Vincter, P. Vogel, D.R. Ward, T. Watari, B.R. Webber, G. Weiglein, J.D. Wells, M. Whalley, A. Wheeler, C.G. Wohl, L. Wolfenstein, J. Womersley, C.L. Woody, R.L. Workman, A. Yamamoto, W.-M. Yao, O.V. Zenin, J. Zhang, R.-Y. Zhu, P.A. Zyla, G. Harper, V.S. Lugovsky, and P. Schaffner. Review of particle physics. Physics Letters B, 667(1-5):1 - 6, 2008. Review of Particle Physics.

[2] B. T. Cleveland, T. Daily, Jr. R. Davis, J. R. Distel, K. Lande, C. K. Lee, P. S. Wildenhain, and J. Ullman. Measurement of the solar electron neutrino flux with the Homestake chlorine detector. The Astrophysical Journal, 496(1):505, 1998.

[3] Y. Fukuda, T. Hayakawa, E. Ichihara, K. Inoue, K. Ishihara, H. Ishino, Y. Itow, T. Kajita, J. Kameda, S. Kasuga, K. Kobayashi, Y. Kobayashi, Y. Koshio, K. Martens, M. Miura, M. Nakahata, S. Nakayama, A. Okada, M. Oketa, K. Okumura, M. Ota, N. Sakurai, M. Shiozawa, Y. Suzuki, Y. Takeuchi, Y. Totsuka, S. Yamada, M. Earl, A. Habig, E. Kearns, S. B. Kim, M. D. Messier, K. Scholberg, J. L. Stone, L. R. Sulak, C. W. Walter, M. Goldhaber, T. Barszczak, W. Gajewski, P. G. Halverson, J. Hsu, W. R. Kropp, L. R. Price, F. Reines, H. W. Sobel, M. R. Vagins, K. S. Ganezer, W. E. Keig, R. W. Ellsworth, S. Tasaka, J. W. Flanagan, A. Kibayashi, J. G. Learned, S. Matsuno, V. Stenger, D. Takemori, T. Ishii, J. Kanzaki, T. Kobayashi, K. Nakamura, K. Nishikawa, Y. Oyama, A. Sakai, M. Sakuda, O. Sasaki, S. Echigo, M. Kohama, A. T. Suzuki, T. J. Haines, E. Blaufuss, R. Sanford, R. Svoboda, M. L. Chen, Z. Conner, J. A. Goodman, G. W. Sullivan, M. Mori, J. Hill, C. K. Jung, C. Mauger, C. McGrew, E. Sharkey, B. Viren, C. Yanagisawa, W. Doki, T. Ishizuka, Y. Kitaguchi, H. Koga, K. Miyano, H. Okazawa, 
C. Saji, M. Takahata, A. Kusano, Y. Nagashima, M. Takita, T. Yamaguchi, M. Yoshida, M. Etoh, K. Fujita, A. Hasegawa, T. Hasegawa, S. Hatakeyama, T. Iwamoto, T. Kinebuchi, M. Koga, T. Maruyama, H. Ogawa, A. Suzuki, F. Tsushima, M. Koshiba, M. Nemoto, K. Nishijima, T. Futagami, Y. Hayato, Y. Kanaya, K. Kaneyuki, Y. Watanabe, D. Kielczewska, R. Doyle, J. George, A. Stachyra, L. Wai, J. Wilkes, and K. Young. Study of the atmospheric neutrino flux in the multi-GeV energy range. Physics Letters B, 436(1-2):33 $-41,1998$.

[4] Q. R. Ahmad, R. C. Allen, T. C. Andersen, J. D.Anglin, J. C. Barton, E. W. Beier, M. Bercovitch, J. Bigu, S. D. Biller, R. A. Black, I. Blevis, R. J. Boardman, J. Boger, E. Bonvin, M. G. Boulay, M. G. Bowler, T. J. Bowles, S. J. Brice, M. C. Browne, T. V. Bullard, G. Bühler, J. Cameron, Y. D. Chan, H. H. Chen, M. Chen, X. Chen, and B. T. Cleveland. Direct evidence for neutrino flavor transformation from neutral-current interactions in the Sudbury Neutrino Observatory. Phys. Rev. Lett., 89(1):011301, Jun 2002.

[5] K. Eguchi, S. Enomoto, K. Furuno, J. Goldman, H. Hanada, H. Ikeda, K. Ikeda, K. Inoue, K. Ishihara, W. Itoh, T. Iwamoto, T. Kawaguchi, T. Kawashima, H. Kinoshita, Y. Kishimoto, M. Koga, Y. Koseki, T. Maeda, T. Mitsui, M. Motoki, K. Nakajima, M. Nakajima, T. Nakajima, H. Ogawa, K. Owada, T. Sakabe, and I. Shimizu. First results from KamLAND: Evidence for reactor antineutrino disappearance. Phys. Rev. Lett., 90(2):021802, Jan 2003.

[6] C. Athanassopoulos, L. B. Auerbach, R. L. Burman, I. Cohen, D. O. Caldwell, B. D. Dieterle, J. B. Donahue, A. M. Eisner, A. Fazely, F. J. Federspiel, G. T. Garvey, M. Gray, R. M. Gunasingha, R. Imlay, K. Johnston, H. J. Kim, W. C. Louis, R. Majkic, J. Margulies, K. McIlhany, W. Metcalf, G. B. Mills, R. A. Reeder, V. Sandberg, D. Smith, I. Stancu, and W. Strossman. Evidence for 
neutrino oscillations from muon decay at rest. Phys. Rev. C, 54(5):2685-2708, Nov 1996.

[7] N. Agafonova, A. Aleksandrov, O. Altinok, M. Ambrosio, A. Anokhina, S. Aoki, A. Ariga, T. Ariga, D. Autiero, A. Badertscher, A. Bagulya, A. Bendhabi, A. Bertolin, M. Besnier, D. Bick, V. Boyarkin, C. Bozza, T. Brugire, R. Brugnera, F. Brunet, G. Brunetti, S. Buontempo, A. Cazes, L. Chaussard, M. Chernyavsky, V. Chiarella, N. Chon-Sen, A. Chukanov, R. Ciesielski, F.Dal Corso, N. D’Ambrosio, Y. Declais, P. del Amo Sanchez, G. De Lellis, M. De Serio, F. Di Capua, A. Di Crescenzo, D. Di Ferdinando, N. Di Marco, A. Di Giovanni, S. Dmitrievsky, M. Dracos, D. Duchesneau, S. Dusini, T. Dzhatdoev, J. Ebert, O. Egorov, R. Enikeev, A. Ereditato, L.S. Esposito, J. Favier, T. Ferber, R.A. Fini, D. Frekers, T. Fukuda, V. Galkin, A. Garfagnini, G. Giacomelli, M. Giorgini, J. Goldberg, C. Gllnitz, D. Golubkov, L. Goncharova, Y. Gornushkin, G. Grella, F. Grianti, A.M. Guler, C. Gustavino, C. Hagner, K. Hamada, T. Hara, M. Hierholzer, K. Hoshino, M. Ieva, H. Ishida, K. Ishiguro, K. Jakovcic, C. Jollet, F. Juget, M. Kamiscioglu, J. Kawada, M. Kazuyama, S.H. Kim, M. Kimura, N. Kitagawa, B. Klicek, J. Knuesel, K. Kodama, M. Komatsu, U. Kose, I. Kreslo, H. Kubota, C. Lazzaro, J. Lenkeit, I. Lippi, A. Ljubicic, A. Longhin, G. Lutter, A. Malgin, G. Mandrioli, K. Mannai, A. Marotta, J. Marteau, T. Matsuo, V. Matveev, N. Mauri, E. Medinaceli, F. Meisel, A. Meregaglia, P. Migliozzi, S. Mikado, S. Miyamoto, P. Monacelli, K. Morishima, U. Moser, M.T. Muciaccia, N. Naganawa, T. Naka, M. Nakamura, T. Nakano, Y. Nakatsuka, D. Naumov, V. Nikitina, K. Niwa, Y. Nonoyama, S. Ogawa, A. Olchevsky, T. Omura, G. Orlova, V. Osedlo, M. Paniccia, A. Paoloni, B.D. Park, I.G. Park, A. Pastore, L. Patrizii, E. Pennacchio, H. Pessard, V. Pilipenko, C. Pistillo, N. Polukhina, M. Pozzato, K. Pretzl, P. Publichenko, F. Pupilli, J.P. Repellin, R. Rescigno, T. Roganova, H. Rokujo, G. Romano, G. Rosa, I. Rostovtseva, A. Rubbia, 
A. Russo, V. Ryasny, O. Ryazhskaya, Y. Sakatani, O. Sato, Y. Sato, A. Schembri, W. Schmidt-Parzefall, H. Schroeder, L. Scotto Lavina, A. Sheshukov, H. Shibuya, S. Simone, M. Sioli, C. Sirignano, G. Sirri, J.S. Song, M. Spinetti, L. Stanco, N. Starkov, M. Stipcevic, T. Strauss, P. Strolin, K. Suzuki, S. Takahashi, M. Tenti, F. Terranova, I. Tezuka, V. Tioukov, P. Tolun, A. Trabelsi, T. Tran, S. Tufanli, P. Vilain, M. Vladimirov, L. Votano, J.-L. Vuilleumier, G. Wilquet, B. Wonsak, V. Yakushev, C.S. Yoon, J. Yoshida, T. Yoshioka, Y. Zaitsev, S. Zemskova, A. Zghiche, and R. Zimmermann. Observation of a first $\nu_{\tau}$ candidate event in the OPERA experiment in the CNGS beam. Physics Letters B, 691(3):138 - 145, 2010.

[8] Z. Maki, M. Nakagawa, and S. Sakata. Remarks on the unified model of elementary particles. Progress of Theoretical Physics, 28(5):870-880, 1962.

[9] B. Aharmim, S. N. Ahmed, J. F. Amsbaugh, A. E. Anthony, J. Banar, N. Barros, E. W. Beier, A. Bellerive, B. Beltran, M. Bergevin, S. D. Biller, K. Boudjemline, M. G. Boulay, T. J. Bowles, M. C. Browne, T. V. Bullard, T. H. Burritt, B. Cai, Y. D. Chan, D. Chauhan, M. Chen, B. T. Cleveland, G. A. Cox-Mobrand, C. A. Currat, X. Dai, H. Deng, and J. Detwiler. Independent measurement of the total active ${ }^{8} \mathrm{~B}$ solar neutrino flux using an array of ${ }^{3} \mathrm{He}$ proportional counters at the Sudbury Neutrino Observatory. Phys. Rev. Lett., 101(11):111301, Sep 2008.

[10] Y. Ashie, J. Hosaka, K. Ishihara, Y. Itow, J. Kameda, Y. Koshio, A. Minamino, C. Mitsuda, M. Miura, S. Moriyama, M. Nakahata, T. Namba, R. Nambu, Y. Obayashi, M. Shiozawa, Y. Suzuki, Y. Takeuchi, K. Taki, S. Yamada, M. Ishitsuka, T. Kajita, K. Kaneyuki, S. Nakayama, A. Okada, K. Okumura, C. Saji, and Y. Takenaga. Measurement of atmospheric neutrino oscillation parameters by Super-Kamiokande I. Phys. Rev. D, 71(11):112005, Jun 2005. 
[11] M. Apollonio, A. Baldini, C. Bemporad, E. Caffau, F. Cei, Y. Dclais, H. de Kerret, B. Dieterle, A. Etenko, J. George, G. Giannini, M. Grassi, Y. Kozlov, W. Kropp, D. Kryn, M. Laiman, C. E. Lane, B. Lefivre, I. Machulin, A. Martemyanov, V. Martemyanov, L. Mikaelyan, D. Nicol, M. Obolensky, R. Pazzi, G. Pieri, L. Price, S. Riley, R. Reeder, A. Sabelnikov, G. Santin, M. Skorokhvatov, H. Sobel, J. Steele, R. Steinberg, S. Sukhotin, S. Tomshaw, D. Veron, and V. Vyrodov. Limits on neutrino oscillations from the chooz experiment. Physics Letters B, 466(2-4):415 - 430, 1999.

[12] P. Adamson, C. Andreopoulos, K. E. Arms, R. Armstrong, D. J. Auty, D. S. Ayres, B. Baller, P. D. Barnes, G. Barr, W. L. Barrett, B. R. Becker, A. Belias, R. H. Bernstein, D. Bhattacharya, M. Bishai, A. Blake, G. J. Bock, J. Boehm, D. J. Boehnlein, D. Bogert, C. Bower, E. Buckley-Geer, S. Cavanaugh, J. D. Chapman, D. Cherdack, S. Childress, and B. C. Choudhary. Measurement of neutrino oscillations with the MINOS detectors in the NuMI beam. Phys. Rev. Lett., 101(13):131802, Sep 2008.

[13] V. M. Lobashev, V. N. Aseev, A. I. Belesev, A. I. Berlev, E. V. Geraskin, A. A. Golubev, O. V. Kazachenko, Yu. E. Kuznetsov, R. P. Ostroumov, L. A. Rivkis, B. E. Stern, N. A. Titov, S. V. Zadorozhny, and Yu. I. Zakharov. Direct search for mass of neutrino and anomaly in the tritium beta-spectrum. Physics Letters B, 460(1-2):227 - 235, 1999.

[14] Ch. Kraus, B. Bornschein, L. Bornschein, J. Bonn, B. Flatt, A. Kovalik, B. Ostrick, E. W. Otten, J. P. Schall, Th. Thmmler, and Ch. Weinheimer. Final results from phase II of the Mainz neutrino mass search in tritium $\beta$ decay. 2004.

[15] D. N. Spergel, L. Verde, H. V. Peiris, E. Komatsu, M. R. Nolta, C. L. Bennett, M. Halpern, G. Hinshaw, N. Jarosik, A. Kogut, M. Limon, S. S. Meyer, L. Page, G. S. Tucker, J. L. Weiland, E. Wollack, and E. L. Wright. First- 
year Wilkinson Microwave Anisotropy Probe (WMAP) observations: Determination of cosmological parameters. The Astrophysical Journal Supplement Series, 148(1):175, 2003.

[16] T. J. Loredo and D. Q. Lamb. Bayesian analysis of neutrinos observed from supernova SN 1987A. Phys. Rev. D, 65(6):063002, Feb 2002.

[17] M. Goeppert-Mayer. Double beta-disintegration. Phys. Rev., 48(6):512-516, Sep 1935.

[18] W. H. Furry. On transition probabilities in double beta-disintegration. Phys. Rev., 56(12):1184-1193, Dec 1939.

[19] E. Caurier, F. Nowacki, A. Poves, and J. Retamosa. Shell model study of the neutrinoless double beta decays. Nuclear Physics A, 654(1, Supplement 1):973c - 976c, 1999 .

[20] J. Engel, P. Vogel, and M. R. Zirnbauer. Nuclear structure effects in doublebeta decay. Phys. Rev. C, 37(2):731-746, Feb 1988.

[21] A. Staudt, K. Muto, and H. V. Klapdor-Kleingrothaus. Calculation of $2 \nu$ and $0 \nu$ double-beta decay rates. EPL (Europhysics Letters), 13(1):31, 1990.

[22] A. Faessler and F. Simkovic. Double beta decay. Journal of Physics G: Nuclear and Particle Physics, 24(12):2139, 1998.

[23] J. Toivanen and J. Suhonen. Renormalized proton-neutron quasiparticle random-phase approximation and its application to double beta decay. Phys. Rev. Lett., 75(3):410-413, Jul 1995.

[24] G. Pantis, F. Šimkovic, J. D. Vergados, and A. Faessler. Neutrinoless double beta decay within the quasiparticle random-phase approximation with protonneutron pairing. Phys. Rev. C, 53(2):695-707, Feb 1996. 
[25] O. A. Rumyantsev and M. H. Urin. The strength of the analog and GamowTeller giant resonances and hindrance of the $2 \nu \beta \beta$-decay rate. Physics Letters $B, 443(1-4): 51-57,1998$.

[26] H. V. Klapdor-Kleingrothaus, A. Dietz, H. L. Harney, and I. V. Krivosheina. Evidence for Neutrinoless Double Beta Decay. Mod. Phys. Lett., A16:2409$2420,2001$.

[27] K. You, Y. Zhu, J. Lu, H. Sun, W. Tian, W. Zhao, Z. Zheng, M. Ye, C. Ching, T. Ho, F. Cui, C. Yu, and G. Jiang. A search for neutrinoless double $\beta$ decay of ${ }^{48}$ Ca. Physics Letters B, 265(1-2):53 - 56, 1991.

[28] H.V. Klapdor-Kleingrothaus, A. Dietz, L. Baudis, G. Heusser, I.V. Krivosheina, B. Majorovits, H. Paes, H. Strecker, V. Alexeev, A. Balysh, A. Bakalyarov, S.T. Belyaev, V.I. Lebedev, and S. Zhukov. Latest results from the HEIDELBERG-MOSCOW double beta decay experiment. Eur. Phys. J. A, 12(2):147-154, 2001.

[29] C. E. Aalseth, F. T. Avignone, R. L. Brodzinski, S. Cebrian, E. Garcia, D. Gonzalez, W. K. Hensley, I. G. Irastorza, I. V. Kirpichnikov, A. A. Klimenko, H. S. Miley, A. Morales, J. Morales, A. Ortiz de Solorzano, S. B. Osetrov, V. S. Pogosov, J. Puimedon, J. H. Reeves, M. L. Sarsa, A. A. Smolnikov, A. S. Starostin, A. G. Tamanyan, A. A. Vasenko, S. I. Vasiliev, and J. A. Villar. IGEX ${ }^{76}$ Ge neutrinoless double-beta decay experiment: Prospects for next generation experiments. Phys. Rev. D, 65(9):092007, May 2002.

[30] S. R. Elliott, A. A. Hahn, M. K. Moe, M. A. Nelson, and M. A. Vient. Double beta decay of ${ }^{82}$ Se. Phys. Rev. C, 46(4):1535-1537, Oct 1992.

[31] H. Ejiri, K. Fushimi, K. Hayashi, T. Kishimoto, N. Kudomi, K. Kume, H. Kuramoto, K. Matsuoka, H. Ohsumi, K. Takahisa, and S. Yoshida. Limits on 
the majorana neutrino mass and right-handed weak currents by neutrinoless double $\beta$ decay of ${ }^{100}$ Mo. Phys. Rev. C, 63(6):065501, Apr 2001.

[32] F. A. Danevich, A. Sh. Georgadze, V. V. Kobychev, B. N. Kropivyansky, A. S. Nikolaiko, O. A. Ponkratenko, V. I. Tretyak, S. Yu. Zdesenko, Yu. G. Zdesenko, P. G. Bizzeti, T. F. Fazzini, and P. R. Maurenzig. New results of ${ }^{116} \mathrm{Cd}$ double $\beta$ decay study with ${ }^{116} \mathrm{CdWO}_{4}$ scintillators. Phys. Rev. $C$, 62(4):045501, Sep 2000.

[33] T. Bernatowicz, J. Brannon, R. Brazzle, R. Cowsik, C. Hohenberg, and F. Podosek. Precise determination of relative and absolute $\beta \beta$-decay rates of ${ }^{128} \mathrm{Te}$ and ${ }^{130}$ Te. Phys. Rev. C, 47(2):806-825, Feb 1993.

[34] A. Alessandrello, C. Brofferio, O. Cremonesi, E. Fiorini, A. Giuliani, A. Nucciotti, M. Pavan, G. Pessina, S. Pirro, E. Previtali, M. Vanzini, L. Zanotti, C. Bucci, and C. Pobes. New experimental results on double beta decay of 130Te. Physics Letters B, 486(1-2):13 - 21, 2000.

[35] R. Luescher, J. Farine, F. Boehm, J. Busto, K. Gabathuler, G. Gervasio, H. E. Henrikson, V. Jrgens, K. Lou, A. Paic, D. Schenker, A. Tadsen, M. Treichel, J. L. Vuilleumier, J. M. Vuilleumier, and H. Wong. Search for $\beta \beta$ decay in 136Xe: new results from the Gotthard experiment. Physics Letters B, 434(34):407 - 414, 1998.

[36] A. De Silva, M. K. Moe, M. A. Nelson, and M. A. Vient. Double $\beta$ decays of ${ }^{100} \mathrm{Mo}$ and ${ }^{150} \mathrm{Nd}$. Phys. Rev. C, 56(5):2451-2467, Nov 1997.

[37] S. R. Elliott and P. Vogel. Double beta decay. Annual Review of Nuclear and Particle Science, 52(1):115-151, 2002.

[38] J. Schechter and J. W. F. Valle. Neutrinoless double- $\beta$ decay in $\mathrm{SU}(2) \times \mathrm{U}(1)$ theories. Phys. Rev. D, 25(11):2951-2954, Jun 1982. 
[39] A. Ali, A. V. Borisov, and D. V. Zhuridov. Probing new physics in the neutrinoless double beta decay using electron angular correlation. Phys. Rev. $D, 76(9): 093009$, Nov 2007.

[40] M. Czakon, J. Gluza, and M. Zralek. Low energy physics and left-right symmetry: bounds on the model parameters. Physics Letters B, 458(2-3):355 360, 1999.

[41] R. DeVoe, A. Dolgolenko, G. Giannini, G. Gratta, P. Picchi, A. Piepke, F. Pietropaolo, J-L. Vuilleumier, P. Vogel, Y-F. Wang, and O. Zeldovich. A very large xenon double-beta decay experiment: Opportunities in science, technology and nuclear non-proliferation. Technical report, 1999.

[42] E. Conti, R. DeVoe, G. Gratta, T. Koffas, S. Waldman, J. Wodin, D. Akimov, G. Bower, M. Breidenbach, R. Conley, M. Danilov, Z. Djurcic, A. Dolgolenko, C. Hall, A. Odian, A. Piepke, C. Y. Prescott, P. C. Rowson, K. Skarpaas, J-L. Vuilleumier, K. Wamba, and O. Zeldovich. Correlated fluctuations between luminescence and ionization in liquid xenon. Phys. Rev. B, 68(5):054201, Aug 2003.

[43] M. Green, J. Wodin, R. DeVoe, P. Fierlinger, B. Flatt, G. Gratta, F. LePort, M. Montero Díez, R. Neilson, K. O'Sullivan, A. Pocar, S. Waldman, D. S. Leonard, A. Piepke, C. Hargrove, D. Sinclair, V. Strickland, W. Fairbank, K. Hall, B. Mong, M. Moe, J. Farine, D. Hallman, C. Virtue, E. Baussan, Y. Martin, D. Schenker, J.-L. Vuilleumier, J.-M. Vuilleumier, P. Weber, M. Breidenbach, R. Conley, C. Hall, J. Hodgson, D. Mackay, A. Odian, C. Y. Prescott, P. C. Rowson, K. Skarpaas, and K. Wamba. Observation of single collisionally cooled trapped ions in a buffer gas. Phys. Rev. A, 76(2):023404, Aug 2007.

[44] H. Dehmelt and P. Toschek. Proposed visual detection laser spectroscopy on 
single $\mathrm{Ba}^{+}$ion. In Bull. Am. Phys. Soc., editor, Anaheim Meeting of the APS, volume 20, page 61, 1975 .

[45] W. Neuhauser, M. Hohenstatt, P. Toschek, and H. Dehmelt. Visual observation and optical cooling of electrodynamically contained ions. Applied Physics A: Materials Science Processing, 17:123-129, 1978. 10.1007/BF00885243.

[46] W. Neuhauser, M. Hohenstatt, P. E. Toschek, and H. Dehmelt. Localized visible Ba+ mono-ion oscillator. Phys. Rev. A, 22(3):1137-1140, Sep 1980.

[47] J. J. Curry. Compilation of wavelengths, energy levels, and transition probabilities for Ba I and Ba II. Journal of Physical and Chemical Reference Data, 33(3):725-746, 2004.

[48] A. Gallagher. Oscillator strengths of Ca II, Sr II, and Ba II. Phys. Rev., 157(1):24-30, May 1967.

[49] J. D. Jackson. Classical electrodynamics. John Wiley \& Sons, Inc., 3rd edition, 1998.

[50] J. Gurell, E. Biémont, K. Blagoev, V. Fivet, P. Lundin, S. Mannervik, L.-O. Norlin, P. Quinet, D. Rostohar, P. Royen, and P. Schef. Laser-probing measurements and calculations of lifetimes of the $5 d^{2} d_{3 / 2}$ and $5 d^{2} d_{5 / 2}$ metastable levels in baII. Phys. Rev. A, 75(5):052506, May 2007.

[51] W. Ruster, J. Bonn, P. Peuser, and N. Trautmann. Sensitive laser spectroscopy on trapped barium ions by quenching with hydrogen. Applied Physics B: Lasers and Optics, 30:83-86, 1983. 10.1007/BF00688481.

[52] A. A. Madej and J. D. Sankey. Quantum jumps and the single trapped barium ion: Determination of collisional quenching rates for the $5 d^{2} d_{5 / 2}$ level. Phys. Rev. A, 41(5):2621-2630, Mar 1990. 
[53] M. T. Bowers. Gas phase ion chemistry: Volume 1, chapter 3. Academic Press, Inc., 1979.

[54] E. W. McDaniel. Atomic Collisions, electron and photon projectiles, chapter 1. John Wiley \& Sons, 1989.

[55] D. Bellert and W. H. Breckenridge. Bonding in ground-state and excitedstate $\mathrm{A}^{+} \cdot \mathrm{Rg}$ van der Waals ions $(\mathrm{A}=$ atom, $\mathrm{Rg}=$ rare-gas atom): $\mathrm{A}$ modelpotential analysis. Chemical Reviews, 102(5):1595-1622, Apr 2002.

[56] A. A. Madej. Private communication.

[57] A. Hermanni and G. Werth. Collisional de-excitation of the metastable Dstates of $\mathrm{Ba}^{+}$by $\mathrm{He}, \mathrm{Ne}, \mathrm{N}_{2}$ and $\mathrm{H}_{2}$. Zeitschrift fr Physik D Atoms, Molecules and Clusters, 11:301-304, 1989. 10.1007/BF01438503.

[58] W. Demtröder. Laser Spectroscopy: Basic Concepts and Instrumentation, chapter 3. Springer, 1996.

[59] M. Zokai, M. Harris, I. Shannon, D. Szebesta, D. McHugh, and E. L. Lewis. Pressure broadening of the $\mathrm{Ba}+$ resonance doublet by helium and argon. Journal of Physics B: Atomic and Molecular Physics, 20(17):4393, 1987.

[60] E. E. Whiting. An empirical approximation to the Voigt profile. Journal of Quantitative Spectroscopy and Radiative Transfer, 8(6):1379 - 1384, 1968.

[61] A. A. Andreev, V. I. Bayanov, V. I. Kryzhanovskii, A. G. Samsonov, and N. A. Solov'ev. Mechanical characteristics of interactions between laser radiation of various wavelengths and opaque materials. Soviet physics. Technical physics, 37(2):158 - 162, 1992.

[62] S. Amoruso, M. Armenante, V. Berardi, R. Bruzzese, G. Pica, and R. Velotta. Chargel species analysis as a diagnostic tool for laser produced plasma characterization. Applied Surface Science, 106:507 - 512, 1996. Proceedings of 
the Second International Conference on Photo-Excited Processes and Applications.

[63] J. C. S. Kools, S. H. Brongersma, E. van de Riet, and J. Dieleman. Concentrations and velocity distributions of positive ions in laser ablation of copper. Applied Physics B: Lasers and Optics, 53:125-130, September 1991.

[64] Jr. C. R. Phipps, T. P. Turner, R. F. Harrison, G. W. York, W. Z. Osborne, G. K. Anderson, X. F. Corlis, L. C. Haynes, H. S. Steele, K. C. Spicochi, and T. R. King. Impulse coupling to targets in vacuum by $\mathrm{KrF}, \mathrm{HF}$, and $\mathrm{CO}_{2}$ single-pulse lasers. Journal of Applied Physics, 64(3):1083-1096, 1988.

[65] M. Abramowitz and I.A. Stegun. Handbook of Mathematical Functions. Dover, New York, fifth edition, 1964.

[66] D.F. Anderson. Measurement of TMAE and TEA vapor pressures. Nuclear Instruments and Methods in Physics Research Section A: Accelerators, Spectrometers, Detectors and Associated Equipment, 270(2-3):416 - 418, 1988.

[67] G.J. Reinis and P.J. Cressman. Tertiary alkylamines as sensitizers for (+) charge injection from selenium into n-Dodecane and as ionic $(+)$ charge carriers in n-dodecane. Journal of Electrostatics, 7:293 - 299, 1979.

[68] J. C. Slater. Atomic radii in crystals. The Journal of Chemical Physics, 41(10):3199-3204, 1964.

[69] B. Aharmim, S. N. Ahmed, A. E. Anthony, E. W. Beier, A. Bellerive, M. Bergevin, S. D. Biller, M. G. Boulay, Y. D. Chan, M. Chen, X. Chen, B. T. Cleveland, G. A. Cox, C. A. Currat, X. Dai, F. Dalnoki-Veress, H. Deng, J. Detwiler, M. DiMarco, P. J. Doe, G. Doucas, P.-L. Drouin, F. A. Duncan, M. Dunford, J. A. Dunmore, E. D. Earle, H. C. Evans, G. T. Ewan, J. Farine, H. Fergani, F. Fleurot, R. J. Ford, J. A. Formaggio, N. Gagnon, 
J. Tm. Goon, K. Graham, E. Guillian, R. L. Hahn, A. L. Hallin, E. D. Hallman, P. J. Harvey, R. Hazama, K. M. Heeger, W. J. Heintzelman, J. Heise, R. L. Helmer, R. J. Hemingway, R. Henning, A. Hime, C. Howard, M. A. Howe, M. Huang, P. Jagam, N. A. Jelley, J. R. Klein, L. L. Kormos, M. Kos, A. Kruger, C. Kraus, C. B. Krauss, T. Kutter, C. C. M. Kyba, H. Labranche, R. Lange, J. Law, I. T. Lawson, K. T. Lesko, J. R. Leslie, J. C. Loach, S. Luoma, R. MacLellan, S. Majerus, H. B. Mak, J. Maneira, A. D. Marino, R. Martin, N. McCauley, A. B. McDonald, S. McGee, C. Mifflin, K. K. S. Miknaitis, M. L. Miller, B. Monreal, B. G. Nickel, A. J. Noble, E. B. Norman, N. S. Oblath, C. E. Okada, H. M. O'Keeffe, G. D. Orebi Gann, S. M. Oser, R. Ott, S. J. M. Peeters, A. W. P. Poon, G. Prior, K. Rielage, B. C. Robertson, R. G. H. Robertson, E. Rollin, M. H. Schwendener, J. A. Secrest, S. R. Seibert, O. Simard, C. J. Sims, D. Sinclair, P. Skensved, R. G. Stokstad, L. C. Stonehill, G. Tesic, N. Tolich, T. Tsui, R. Van Berg, R. G. Van de Water, B. A. VanDevender, C. J. Virtue, T. J. Walker, B. L. Wall, D. Waller, H. Wan Chan Tseung, D. L. Wark, J. Wendland, N. West, J. F. Wilkerson, J. R. Wilson, J. M. Wouters, A. Wright, M. Yeh, F. Zhang, and K. Zuber. A search for neutrinos from the solar hep reaction and the diffuse supernova neutrino background with the Sudbury Neutrino Observatory. The Astrophysical Journal, 653(2):1545, 2006.

[70] J. Bahcall. Software and data for solar neutrino research. http://www.sns . ias.edu/ jnb/SNdata/sndata.html, 2010.

[71] T.-S. Park, L. E. Marcucci, R. Schiavilla, M. Viviani, A. Kievsky, S. Rosati, K. Kubodera, D.-P. Min, and M. Rho. Parameter-free effective field theory calculation for the solar proton-fusion and hep processes. Phys. Rev. C, 67(5):055206, May 2003.

[72] J. N. Bahcall, M. H. Pinsonneault, and S. Basu. Solar models: Current 
epoch and time dependences, neutrinos, and helioseismological properties. The Astrophysical Journal, 555(2):990, 2001.

[73] S. Nakamura, T. Sato, S. Ando, T. S. Park, F. Myhrer, V. Gudkov, and K. Kubodera. Neutrino-deuteron reactions at solar neutrino energies. Nuclear Physics A, 707(3-4):561 - 576, 2002.

[74] S. Nakamura, T. Sato, V. Gudkov, and K. Kubodera. Neutrino reactions on the deuteron. Phys. Rev. C, 63(3):034617, Feb 2001.

[75] A. Kurylov, M. J. Ramsey-Musolf, and P. Vogel. Radiative corrections in neutrino-deuterium disintegration. Physical Review C, 65:055501, 2002.

[76] G. J. Feldman and R. D. Cousins. Unified approach to the classical statistical analysis of small signals. Phys. Rev. D, 57(7):3873-3889, Apr 1998. 